FORSCHUNGSERGEBNISSE DER WIRTSCHAFTSUNIVERSITÄT WIEN

Sarah Meisenberger

\title{
Strukturierte \\ Organisationen und \\ Wissen
}


FORSCHUNGSERGEBNISSE DER

WIRTSCHAFTSUNIVERSITÄT WIEN

Sarah Meisenberger

\section{Strukturierte Organisationen und Wissen}

Betrachtet man aktuelle Literatur aus dem Gebiet der Organisationsforschung beziehungsweise generell Beiträge aus dem Gebiet der Unternehmensführung, erkennt man sehr schnell, dass einem Thema große Bedeutung zukommt - Wissen. Vor dem Hintergrund dieser Feststellung wird versucht, einen theoretischen Wissensmanagement-Ansatz zu elaborieren, im Rahmen dessen untersucht wird, auf welche Weise Unternehmen gestaltet werden sollten, um das Fundament für ein erfolgreiches Wissensmanagement zu bilden. Im Zuge dessen zeigt es sich von Interesse, welche Rolle dem Individuum beigemessen werden sollte und welche strukturellen Änderungen in einem Unternehmen notwendig wären, um das ihnen inhärente Wissen im Sinne der jeweiligen Unternehmensziele zu verwenden.

Sarah Meisenberger war von 2001 bis 2005 Vertragsassistentin an der Abteilung für Public Management des Instituts für Unternehmensführung an der Wirtschaftsuniversität Wien, seit 2005 ist sie Universitätslektorin an gleichnamiger Abteilung. 2001 wurde die Autorin durch eine Marketingberatungsgesellschaft im Rahmen des Wettbewerbs Tuition Waiver 2001 als eine der fünf besten Betriebswirtinnen in Österreich ausgezeichnet. Im Jahr 2004 schloss sie ihre Promotion mit Auszeichnung ab. 
Strukturierte Organisationen

und Wissen

Sarah Meisenberger - 978-3-631-75453-5

Downloaded from PubFactory at 01/11/2019 04:39:57AM

via free access 


\section{Forschungsergebnisse der Wirtschaftsuniversität Wien}

Band 9

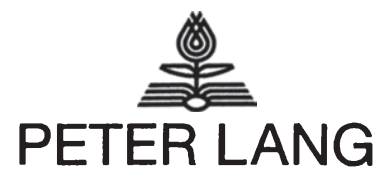

Frankfurt am Main · Berlin · Bern · Bruxelles · New York · Oxford · Wien

Sarah Meisenberger - 978-3-631-75453-5

Downloaded from PubFactory at 01/11/2019 04:39:57AM

via free access 
Sarah Meisenberger

\section{Strukturierte Organisationen und Wissen}

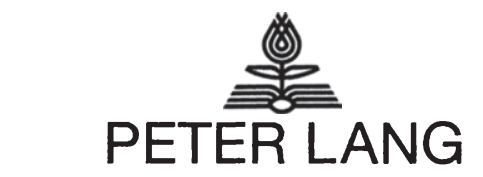

Europäischer Verlag der Wissenschaften

Sarah Meisenberger - 978-3-631-75453-5

Downloaded from PubFactory at 01/11/2019 04:39:57AM

via free access 


\title{
Bibliografische Information Der Deutschen Bibliothek
}

Die Deutsche Bibliothek verzeichnet diese Publikation in der Deutschen Nationalbibliografie; detaillierte bibliografische Daten sind im Internet über <http://dnb.ddb.de> abrufbar.

Open Access: The online version of this publication is published on www.peterlang.com and www.econstor.eu under the international Creative Commons License CC-BY 4.0. Learn more on how you can use and share this work: http://creativecommons.org/ licenses/by/4.0.

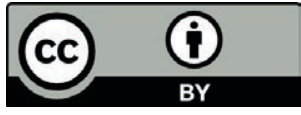

This book is available Open Access thanks to the kind support of ZBW - Leibniz-Informationszentrum Wirtschaft.

Gefördert durch die Wirtschaftsuniversität Wien.

Gedruckt auf alterungsbeständigem, säurefreiem Papier.

\author{
ISSN 1613-3056 \\ ISBN 3-631-53861-8 \\ ISBN 978-3-631-75453-5 (eBook) \\ (c) Peter Lang $\mathrm{GmbH}$ \\ Europäischer Verlag der Wissenschaften \\ Frankfurt am Main 2005 \\ Alle Rechte vorbehalten.
}

Das Werk einschließlich aller seiner Teile ist urheberrechtlich geschützt. Jede Verwertung außerhalb der engen Grenzen des Urheberrechtsgesetzes ist ohne Zustimmung des Verlages unzulässig und strafbar. Das gilt insbesondere für Vervielfältigungen, Übersetzungen, Mikroverfilmungen und die Einspeicherung und Verarbeitung in elektronischen Systemen.

Printed in Germany 123457

www.peterlang.de 


\section{Meiner Familie}

Sarah Meisenberger - 978-3-631-75453-5

Downloaded from PubFactory at 01/11/2019 04:39:57AM

via free access 
Sarah Meisenberger - 978-3-631-75453-5

Downloaded from PubFactory at 01/11/2019 04:39:57AM

via free access 


\section{INHALTSVERZEICHNIS}

I Problemstellung und Gang der Arbeit

1. Problemstellung 9

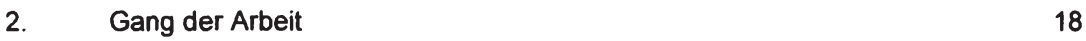

II Wissensmanagement haben oder sein? $\quad 22$

3. Theoretische Positionierung und Grundanlage der Arbeit 22

3.1. Wissen - DIE Ressource oder Resultat von Interaktion? 32

3.2. Management - der Navigator eines Unternehmens? 42

3.3. Wissen managen - eine symbiotische Beziehung oder ein

$\begin{array}{ll}\text { Widerspruch in sich? } & 47\end{array}$

3.4. Exkurs: Organisationales Lernen 60

4. Wissensmanagement haben 67

4.1. Überblick 67

4.2. Die Wissensspirale 70

4.3. K-Worter 76

4.4. Ganzheitliches Wissensmanagement 81

4.5. Die acht Bausteine des Wissensmanagements 86

4.6. Die Wissenstreppe 92

4.7. Das Schichtenmodell 96

4.8. Lebenszyklusmodell des Wissensmanagements 101

4.9. Abschließende Betrachtung 106 
III Wissensmanagement in Anlehnung an die Neue Institutionenokonomie

109

5. Die Neue Institutionenökonomie 109

5.1. Überblick 109

5.2. Institutionen - die Spielregeln von Organisationen 118

5.3. Teiltheorien der Neuen Institutionenokonomie 123

5.3.1. Die Transaktionskostentheorie $\quad 125$

$\begin{array}{ll}\text { 5.3.1.1. Theoretisches Rahmenkonzept } & 125\end{array}$

5.3.1.2. Gestaltungspotentiale 130

5.3.2. Die Theorie der Verfügungsrechte 135

5.3.2.1. Theoretisches Rahmenkonzept $\quad 135$

$\begin{array}{ll}\text { 5.3.2.2. Gestaltungspotentiale } & 138\end{array}$

5.3.3. Die Agenturtheorie 142

5.3.3.1. Theoretisches Rahmenkonzept 142

$\begin{array}{ll}\text { 5.3.3.2. Gestaltungspotentiale } & 147\end{array}$

5.4. Abschließende Betrachtung 151

6. Ein Entwurf eines Wissensmanagement-Ansatzes 155

6.1. Überblick 155

6.2. Handlungsrelevantes Wissen 159

6.3. Organisation - Ordnung und Struktur verleihen 164

6.4. Die Rolle des Individuums 183

6.5. Kommunikation - der Schlüsselfaktor 189

6.6. Wissen verfeinern - Qualitătsmanagement 198

6.7. Technologische Untermauerung 205

6.8. Abschließende Betrachtung 212

$\begin{array}{ll}\text { IV Weiterfürende Gestaltungsoptionen } & 216\end{array}$

$\begin{array}{lll}V & \text { Zusammenfassung und Ausblick } & 221\end{array}$

$\begin{array}{ll}\text { VI Literaturverzeichnis } & 224\end{array}$ 


\section{Problemstellung und Gang der Arbeit}

\section{Problemstellung}

Betrachtet man aktuelle Literatur aus dem Gebiet der Organisationsforschung bzw. generell Beiträge aus dem Gebiet der Unternehmensführung, erkennt man sehr schnell, dass einem Thema große Bedeutung zukommt - Wissen (vgl. Blackler, 1995, Probst/Büchel, 1997, von Krogh/Roos/Kleine, 1998, Gherardi, 1996). Wissen wird oftmals per se behandelt, aber oft in Zusammenhang mit anderen Phänomenen, wie beispielsweise der Wissensgesellschaft (Willke, 2001), den Wissensarbeitern (Drucker, 1993), dem wissensbasierten Unternehmen (Spender, 2001) oder der wissensbasierten Unternehmensführung (North, 2002). Bereits mit den Werken Zuboffs (1988) ("In the Age of the Smart Machine") oder Druckers (1993) („Post-Capitalist Society") wurde die akademische Welt bezüglich des Beginns eines neuen Zeitalters sensibilisiert - des Informationszeitalters, wie Lash (1994) konstatierte oder der Entstehung der postindustriellen Gesellschaft, geprägt durch Bell (1973) und eben Drucker (1993). Im Laufe der neunziger Jahre kam es folglich zu einer regelrechten Publikationsflut. Immer mehr festigte sich nicht nur in der Wissenschaft, sondern auch vor allem in der betrieblichen Praxis das Verständnis, dass Wissen einen Faktor darstellt, dem eine besondere Bedeutung zugesprochen werden sollte. Es manifestierte sich ein Einfluss des Konzeptes Wissen, der sich nicht nur auf Wirtschaftstheorien oder auf das wirtschaftliche Umfeld, sondern auch auf gesellschaftliche Bereiche entfaltete. In Form eines Common Sense herrscht Konsens darüber, dass Wissen eine einzigartige Ressource bzw. einen einzigartigen Produktionsfaktor darstellt, vielleicht sogar den bedeutendsten, der die klassische Dreierkombination an Ressourcen: „Kapital, Personen und Land“ erweitert und der sie sogar in ihrer Bedeutung verdrängen mag (vgl. Bell, 1976; Drucker, 1993; Willke, 1999). Darauf aufbauend werden die verschiedensten Fragestellungen, sei es auf sozialer, technischer oder rein administrativer Ebene, zu beantworten versucht. 
Die fortwährenden Errungenschaften in den Informations- und Kommunikationstechnologien bieten Unternehmen die Chance, ihren Gestaltungsspielraum zu erweitern, wie sie ihre Unternehmensstrukturen und Austauschbeziehungen besser organisieren können. „Besser" bedeutet in diesem Sinne, dass die unternehmensweit verfügbaren Ressourcen ihrem Entsprechen nach eingesetzt und genutzt werden und dass dies durch die Unterstützung von IT-Medien und/oder neuartigen Managementkonzepten optimiert wird. Diese fortwährende Weiterentwicklung der Managementlösungen vollzieht sich nicht auf organisationaler Ebene, sondern auf einer Metaebene, die eben erst die Veränderungen auf organisationaler Ebene induziert. Somit kommt es im Unternehmen zu einer Veränderung der kollektiv durchgeführten Handlungen innerhalb der bestehenden Normen, Werte und Strukturen, was auch als "Single-loop-Learning" in den Management-Fachjargon eingegangen ist (Argyris/Schön, 1978). Andererseits werden vor allem von der Praxis Problemstellungen an die Wissenschaft herangetragen, die es zu lösen gilt, um diesen Problembereichen mittels "neuer" bzw. weiterentwickelter Konzepte Rechnung tragen zu können. Einer derartigen Überarbeitung ist inhärent, dass existierende Lösungsansätze hinterfragt werden, was oftmals auch zu einer kritischen Durchleuchtung von Wertvorstellungen und Normen führt. Es kommt zu einem so genannten „Double-loop-Learning", das als eine kritische Überprüfung der und Veränderung von den zugrunde gelegten interpretativen Mechanismen, Zielen und Annahmen verstanden wird (Argyris/Schön, 1978).

Nicht nur Individuen werden durch die steigende Bedeutung von Wissen und Wissensmanagement beeinflusst. Auch auf Organisationen entfaltet dieser Bedeutungszuwachs einen starken Einfluss, betrachtet man nur die Weiterentwicklung der Managementkonzepte und Managementtechniken der letzten Jahre. Eine Fülle an Programmen und neuen Managementtechnologien wurde entwickelt, die zum überwiegenden Teil ein gemeinsames Ziel haben, nämlich die Wettbewerbsbedingungen für Unternehmen optimal zu gestalten und durch effektive Organisationsstrukturen Unternehmen effizienter zu machen, damit sie am Markt eine bessere Positionierung erreichen und besser überleben können. 
Auf der einen Seite analysieren dafür Strategen fortwährend die bestehenden Ressourcen und Märkte ihres Unternehmens. Dabei wird relevantes Wissen im Unternehmen sowie Unternehmensumfeld identifiziert, um es im Sinne des Unternehmens einzusetzen. Neue Möglichkeiten auf Märkten müssen gefunden werden, mit Hilfe derer Ressourcen und Wissen angesammelt werden können, wobei veraltete Routinen und Handlungsabläufe nicht weiter durchgeführt werden. Auf der anderen Seite sieht sich das strategische Management der Herausforderung gegenüber, neues Wissen aufzubauen, um für die Zukunft einen komparativen Vorteil zu generieren (Hamel/Prahalad, 1994). Das Gestalten einer derartigen Kernkompetenz als ein Mix an Skills und Technologien (vgl. Prahalad/ Hamel, 1990) "is a way of behaving, indeed a way of being, in which everyone is a knowledge worker - that is to say, an entrepreneur" (Nonaka, 1994).

Im Rahmen derartiger Bemühungen wurden beispielsweise Konzepte wie "LeanManagement", „Business-Process Reengineering", "Total Quality Management", die "Balanced Scorecard" sowie Überlegungen zu "lernenden Organisationen" etc. entwickelt. Viele dieser Konzepte dominierten jedoch nur kurzzeitig die Unternehmerwelt, da sie beim Versuch ihrer Verwirklichung praktisch nicht bzw. nicht ausreichend realisierbar waren.

Auch ein scheinbar "neuer" Trend - Wissensmanagement - wurde in dieser Entwicklungsphase von zahlreichen Disziplinen aufgegriffen. Dementsprechend vielschichtig und heterogen präsentiert sich die dazugehörige Forschungslandschaft. So findet man Auseinandersetzungen mit dieser Thematik beispielsweise in der Organisations- und Managementforschung (vgl. Walsh/ Ungeson, 1991; Probst et al., 1997; Davenport/Prusak, 1998), den Informatikwissenschaften (vgl. Earl, 1996; Faisst, 1996, in: Neumann, 2000; Roehl, 2000; Boland/Tankasi, 1995), der Wissenssoziologie (vgl. Willke, 1998; Knorr-Cetina, 1999) oder der Strategielehre (vgl. Spender, 1996; Grant, 1996; von Krogh, 1998).

Untersucht man die behandelten Themenstellungen im Bereich Wissensmanagement jedoch genauer, wird man unschwer erkennen, dass das Management von 
Wissen nichts Neues ist (Zand, 1969; Rickson, 1976; Zuboff, 1988). Im Grunde muss jedes erfolgreiche Unternehmen wissensorientiert agieren, um die internen Ressourcen optimal einzusetzen, da die Wettbewerbsfähigkeit von Unternehmen unter anderem auf der Fähigkeit beruht, kollektives betriebliches Wissen kontinuierlich zu schaffen und zu verbinden (Brown/Duguid, 1999; Hamel/ Prahalad, 1994).

Auch ist die Auseinandersetzung mit "Wissen" an sich so alt wie die Philosophie selbst, wobei der Einfluss auf Organisations- und Führungstheorien erst im Laufe des 20. Jahrhunderts als derartig markant konstatiert werden kann, betrachtet man die Publikationen in reviewed Journals. Im Rahmen dieser Arbeit wird auf eine Aufarbeitung der vollständigen Historie des Begriffs "Wissen" verzichtet. Ein chronologischer Rückblick und eine Darstellung von Vertretern mitsamt ihren Grundaussagen, die die Philosophie des jeweils behandelten Zeitabschnitts unbestritten stark beeinflussten, finden sich beispielsweise bei Spierling (2002), VanDoren (1996).

Eine Erwähnung sollen diejenigen Philosophen erfahren, auf die bei der Definierung des Begriffs "Wissen" im Rahmen der Sozial- und Wirtschaftswissenschaften rekurriert wird. So entfaltet beispielsweise der Diskurs zwischen den Vertretern des Rationalismus und Empirismus, der im 17. und 18. Jahrhundert die Philosophie zweiteilte, Einfluss auf diverse Ausführungen im Bereich Wissen.

Vertreter des Rationalismus stützen sich auf die Vernunft, wobei diese von sich aus und weitgehend unabhängig von Sinneswahrnehmungen das Wesen der Dinge rein begrifflich bestimmen kann. Als Hauptvertreter dieser Strömung können Descartes, Spinoza, Leibniz und Wolff genannt werden. Descartes beispielsweise versucht eine mathematische Erkenntnisgewissheit und stellt in seiner streng dualistisch konzipierten Zweiweltentheorie der rein geistigen Welt eine rein körperlich ausgedehnte Welt gegenüber. Letztere kann auf Grund von Gesetzen, die auf einer auf Ursache-Wirkungszusammen-hängen aufbauenden Mechanik basieren, erfasst werden (Spierling 20P2: VanDoren 1996) 
Im Gegensatz dazu stehen die Empiristen, die auf der Erfahrung aufbauen und alles das, was nicht durch sinnliche Wahrnehmungen belegt werden kann und Tatsachen widerspiegelt, nicht als wirklich oder für nicht erkennbar erachten, was deutlich eine der Metaphysik gegenüber teilweise ablehnende Haltung aufzeigt. Hier findet man Vertreter wie Bacon, Locke, Berkeley oder Hume (Spierling, 2002; VanDoren, 1996).

Diese Zeit, als Jahrhundert der Aufklärung gefeiert, wird unter anderem durch die Beiträge von Immanuel Kant geprägt. Dieser streicht die Würde des Menschen und das sittliche Handeln hervor, das aus der Idee der Würde eines vernünftigen Wesens, das keinem Gesetz gehorcht als dem, das es zugleich selbst gibt, entsteht. In seiner mathematisch-naturwissenschaftlich orientierten Erkenntnistheorie erklärt Kant die Metaphysik im traditionellen Sinne (das erfahrungs-unabhängige Wissen über das Weltganze und Gott) als beendet. Wir selbst können die Dinge, die außerhalb und unabhängig von unserer Erkenntnis bestehen, nicht erkennen und somit stoßen wir auf unsere Grenzen des Wissens, wo das Nichtwissen seinen Anfang nimmt (Kant, 1971).

Bereits ein Jahrhundert später kommt es zu einer Verweltlichung der Philosophie. Durch die industrielle Revolution angetrieben, wird der Versuch, die Welt über metaphysische Ansätze zu erklären, fast gänzlich aufgegeben und es kommt zu einer Vorherrschaft der Naturwissenschaften und der Technik. So festigen sich zu dieser Zeit neue Strömungen, wie beispielsweise der Positivismus, der seinen Ausgangspunkt im „Positiven" sieht und sich auf die Zusammenfassung von naturwissenschaftlichen Tatsachen beschränkt (Spierling, 2002, VanDoren, 1996).

Sich vom Positivismus abgrenzend gestaltet sich der kritische Rationalismus unter Popper, eine Wissenschaft, die jeglichen Dogmatismus verneint. Darunter wird subsumiert, dass jegliche wissenschaftstheoretischen Stellungnahmen nur vorläufig sein können, weil ihnen ein Verbesserungspotential inhärent ist, das durch die Falsifikation aufgezeigt wird (Popper, 1971). 
Wie jedoch "Wissen" definiert werden sollte und welche Bedeutung es in bzw. welchen Einfluss es auf Organisationen ausübt, darüber ist sich die Forschung in keinster Weise einig. Es gibt zahlreiche Ansätze und Interpretationsmöglichkeiten (vgl. Venzin, von Krogh, Roos, 1998; Griseri, 2002; Spender, 1996; Schick, 2002; Blosch, 2001), was oftmals einen Vergleich bzw. eine Kompatibilität der Ergebnisse unterbindet und die Argumentationen in dem Forschungsbereich des Wissensmanagements als sehr inkonsistent erscheinen lässt. Je nach theoretischer Ausrichtung werden aus dem jeweiligen Blickwinkel der Wissenschafter unterschiedliche Forschungsgegenstände, Erkenntnisziele und Methoden ausgemacht. Die epistemologische Ausrichtung der jeweiligen Autoren weist darauf hin, wie sie die Welt verstehen versuchen und Wissen ergründen. Kann Wissen als zu Individuen externe Ressource vorhanden sein oder gestaltet sich Wissen durch die fortwährende Interaktion von Individuen, die ihre soziale Wirklichkeit konstruieren? Eine Übersicht über bearbeitete Wissensthematiken im Bereich der wissensorientierten Unternehmensführung findet sich beispielsweise bei Neumann $(2000$, S. 24-25).

Je nach der Zuordnung zu einem Forschungsprogramm gestalten sich wissenschaftliche Ausführungen. In der Organisationsforschung kann festgestellt werden, dass ein funktionalistisches Paradigma über ein interpretatives dominiert (vgl. Scherer (1998), S. 15). Ersteres basiert auf einem analytischen Zugang und auf der Idee einer ontologisch gegebenen und vollständig beschreibbaren Realität. Theorien, die sich letzterem Paradigma zugehörig fühlen, stellen den Status und die Existenz einer objektiv gegebenen Realität infrage und sehen diese als eine soziale Konstruktion, die von einer bestimmten Gruppe geteilt wird, nicht aber notwendig auch von anderen (vgl. Morgan, 1980, S. 619, in Ebers, 1985).

Dass eine gewisse epistemologische Ausrichtung bestimmte methodologische, ontologische sowie methodische Positionierungen impliziert, ist evident. So kann auch die Organisationsforschung nicht als ein in sich geschlossenes Forschungsprogramm vorgefunden werden, sondern stellt sich mit perspektivischen Verfärbungen dar. Das grundlegende Unterscheidungskriterium erschließt sich in 
der Art und Weise in der der Gegenstandsbereich Organisation konzeptualisiert, methodisch erforscht und, vor allem erklärt wird. Die Grenzen zwischen Forschungsprogrammen werden nicht lediglich hinsichtlich des jeweils von innen postulierten Erklärungsmusters gezogen, sondern markieren gleichzeitig Grenzen zwischen philosophischen Grundpositionen in den Sozialwissenschaften (vgl. Ebers, 1985). In dieser Arbeit wird ein Hinterfragen der philosophischen Ausrichtungen nicht geleistet werden; es wird auch keine Diskussion in diese Richtung gestartet. Vielmehr sollen die Folgen philosophischer Fundierungen im Bereich der Organisationsforschung auf das Forschungsgebiet des Wissensmanagements untersucht werden.

Die zugrunde gelegte organisationstheoretische Ausrichtung führt zu wissenschaftstheoretischen Implikationen, von denen die Betrachtungsweisen von Wissensmanagement abhängig zeichnen. So kann einerseits ein Unternehmen eine Organisation haben, wodurch die Organisation als ein Instrument der Unternehmenspolitik, als Mittel zum Zweck erfolgreicher Unternehmensführung gesehen wird. Es steht die gemeinsame Aufgabenerfüllung von Mensch und Sachmittel im Vordergrund. Die Organisation gestaltet sich als ein Regelsystem der Unternehmensführung zur bestmöglichen Zielerreichung und Sicherstellung von Ergebnissen (vgl. Beiträge im Bereich des Scientific Managements). Ein Wissensmanagement würde sich in einem solchen theoretischen Rahmen ebenfalls als ein Instrument der Unternehmenspolitik eingliedern, mit Hilfe dessen eine noch bessere Zielerreichung erfolgen soll. Werden andererseits Unternehmen als zielorientierte soziale Gebilde betrachtet, so sind sie eine Organisation und im Vordergrund steht die Konstruktion und Gestaltung sozialer Wirklichkeit. Organisationen sind zielgerichtete Handlungssysteme mit interpersonaler Arbeitsteilung (vgl. Kieser/Kubicek, 1992) und die Menschen werden als Träger der Organisation verstanden, die eine Institution im Sinne von organisierten sozialen Zusammenschlüssen schaffen. Wissensmanagement würde sich in einer solchen theoretischen Konzeption als Ergebnis von Interaktions- und Interpretationsprozessen einordnen. 
Obwohl die organisationstheoretische Bedeutung von Wissensmanagement durch eine Publikationsflut in den letzten Jahren in diesem Forschungsbereich sowie durch die stattgefundenen und stattfindenden nationalen sowie internationalen Konferenzen und die zahlreichen Beiträge in reviewed Journals gezeigt werden kann, finden sich vermehrt auch empirische Erhebungen und Studien, die zwar nachweisen, dass Wissensmanagement ein Bedarf zugesprochen wird, jedoch die praktische Umsetzung hinterherhinkt und die Praxis des Wissensmanagements kaum über Versuche der Einführung und fallweisen Anwendung diverser Informations- und Kommunikationstechnologie-Applikationen (wie bspw. Intranet, Data-Warehousing, Groupware oder Wissensdatenbanken) zum Austausch und zur Speicherung individuellen Wissens hinausreichen (vgl. North/ Papp, 1999).

Es ist von Interesse, wie es zu einer solchen Situation kommen mag. Trotz einer Fülle an gebotenen Wissensmanagement-Ansätzen und Forschungsarbeiten in diesem Bereich, scheinen die Forderungen der Praxis nach neuen praktikablen Lösungsansätzen, die den Umgang mit Wissen erleichtern helfen, nicht ausreichend bzw. nur marginal erfüllt zu werden (vgl. Becker, 2001). Wie soll ein Unternehmen betrachtet werden, wenn Wissen gemanagt werden soll? Zahlreiche verschiedenartigste Organisationstheorien bieten Ansatzmöglichkeiten, jedoch jede impliziert für sich eine unterschiedliche Herangehensweise an Wissen selbst. Die Gestaltungsart von Unternehmen (sie sind oder haben ein Wissensmanagement) lässt noch keinen Rückschluss darüber $\mathrm{zu}$, auf welche Art und Weise ein Wissensmanagement aufgebaut werden soll. Welchen Kriterien soll ein Wissensmanagement genügen? Dem zugrunde liegend stellen sich Fragen, wo Wissen gespeichert wird bzw. auffindbar ist? Welche Bedeutung soll dem Individuum im Rahmen eines Wissensmanagements zugesprochen werden? Wie kann Wissen für ein Unternehmen verfügbar gemacht werden? Was kann und sollte ein Unternehmen mit dem zur Verfügung stehenden Wissen tun?

Vor dem Hintergrund der Problemstellung dieser Arbeit kann für die vorliegende Forschungsfrage aufgrund der Komplexität der Themenbereiche "Wissen" und 
"Management" keine universale einheitliche Antwort angestrebt werden. Vielmehr wird versucht, einen theoretischen Wissensmanagement-Ansatz zu elaborieren, im Rahmen dessen untersucht werden soll, auf welche Weise Unternehmen gestaltet werden sollten, um das Fundament für ein erfolgreiches Wissensmanagement zu bilden. Im Zuge dessen zeigt es sich von Interesse, welche Rolle dem Individuum beigemessen werden sollte und welche Handlungen von Unternehmen hinsichtlich des Aufbauens von Anreiz- und Sanktionsmechanismen erwartet werden sollten, um zu gewährleisten, dass Mitarbeiter das innen inhärente Wissen im Sinne der jeweiligen Unternehmensziele verwenden. 


\section{Gang der Arbeit}

Die vorliegende Arbeit verfolgt die theoretische Zielsetzung der Entwicklung eines Entwurfs eines möglichen Wissensmanagement-Ansatzes, dem die Theorie der Neuen Institutionenökonomie zugrunde liegt, und erfährt dabei eine Fünfteilung. Teil I setzt sich mit der Problemstellung (Kapitel 1) sowie dem Gang der Arbeit (Kapitel 2) auseinander, auf die aufbauend die Erörterungen und Analysen erfolgen.

Da eine wissenschaftstheoretische Verortung erforderlich ist, um ein theoretisches Grundgerüst dessen abzustecken, was inhaltlich die Forschungsergebnisse markant beeinflusst, kommt es auch in der vorliegenden Arbeit zu einer theoretischen Positionierung (Teil II). Aufgrund des sehr fragmentierten Feldes des Forschungsbereichs Wissensmanagement und des Vorliegens einer Vielfalt von unterschiedlichsten Wissensmanagement-Ansätzen mit stark divergierenden Grundannahmen erfordert die Elaborierung eines eigenständigen Ansatzes die Analyse der gängigsten Wissensmanagement-Ansätze, die im Bereich der Betriebswirtschaft entwickelt wurden. Aus der Vielzahl der vorliegenden Systematisierungsversuche gängiger Organisationstheorien wird hier das Framework von Burrell/Morgan (1979) als Ausgangspunkt verwendet (Kapitel 3), um einen Überblick über diverse wissenschaftstheoretische Verortungen einzelner Wissensmanagement-Ansätze zu erhalten und das Forschungsfeld Wissensmanagement strukturiert darzustellen.

So soll die Systematisierung von Burrell/Morgan (1979) die Aufarbeitung des Forschungsbereichs unterstützen. Einerseits wird Wissensmanagement in seine Teilthematiken aufgegliedert. So werden die Bereiche "Wissen", "Management" sowie "Wissen managen" getrennt voneinander erläutert (Kapitel 3). Um einen runden Überblick über den State-of-the-Field des Wissensmanagements zu erhalten, werden diese Teilbereiche, trotz einer Verortung der vorliegenden Arbeit in einem funktionalistischen Paradigma, aus der Sicht einer funktionalistischen sowie interpretativen Logik gegenübergestellt. 
Andererseits werden die in der betriebswirtschaftlichen Literatur sieben gängigsten Wissensmanagement-Ansätze dargestellt, die sich einem funktionalistischen Paradigma zuordnen lassen. Sie umfassen "die Wissensspirale" von Nonaka/ Takeuchi (bspw. 1995; 1997; 2000), den Ansatz der „K-Wörter" von DavenportPrusak (1998), den Ansatz des "ganzheitlichen Wissensmanagements" von Albrecht (1997), die „acht Bausteine des Wissensmanagements" von Probst/ Raub/ Romhardth (1997), "die Wissenstreppe" von North (2002), "das Schichtenmodell" von Pautzke (1989) sowie den Ansatz "das Lebenszyklusmodell des Wissensmanagements" von Rehäuser/Krcmar (1996) (Kapitel 4).

Mit der Aufarbeitung des Forschungsfelds Wissensmanagement können Unzulänglichkeiten der dargestellten Ansätze aufgezeigt werden, sodass die eigenständige Entwicklung eines Wissensmanagement-Ansatzes eingeleitet wird (Teil III). Es zeigt sich vor allem von Interesse, auf welche Weise Organisationen gestaltet werden sollten, damit Mitarbeiter auf eine Weise koordiniert und motiviert sind, dass sie ihr Wissen im Sinne von festgelegten Unternehmenszielen verwenden und dieses auf die Erreichung eines gesamtunternehmerischen Zweckes ausrichten. Es gilt eine Organisationstheorie zugrunde zu legen, die hierfür ein Fundament bieten kann.

Hier wird zunächst davon ausgegangen, dass die Institutionenökonomie (New Institutional Economics oder NIE) ein entsprechendes theoretisches Fundament zu liefern imstande ist (Kapitel 5). Die dieser Theorie zugrunde liegenden Annahmen des methodologischen Individualismus und der beschränkten Rationalität, das angebotene Handlungskonzept sowie das Makro-Mikro-MakroSchema sollen auf ihre mögliche Anwendbarkeit hinsichtlich der Elaborierung eines Wissensmangement-Ansatzes untersucht werden.

Die Theorie der Institutionenökonomie befasst sich mit zwei basalen Fragestellungen. Einerseits wird großes Augenmerk auf die Analyse gelegt, welche institutionellen Arrangements die niedrigsten Kosten verursachen und folglich zu einer hohen Effizienz führen, wenn es Koordinationsprobleme zu handhaben gilt. An- 
dererseits wird die Frage aufgeworfen, inwiefern Koordinationsproblematiken, damit einhergehende Kosten sowie die Effizienz von wirtschaftlichem Austausch institutionellen Wandel beeinflussen. Das dieser Theorie zugrunde liegende Konzept der Institution nimmt seinen Ausgangspunkt bei der Betrachtung des Individuums und dessen Handlungen (Ebers/Gotsch, 1999).

Die Theorie der Institutionenökonomie bedient sich in diesem Zusammenhang der Annahmen von Rational-Choice-Modellen. Obwohl Akteure sich einigen sich immer wiederholenden Situationen gegenübersehen, die sie mit rationalem Verhalten handhaben können, sind sie auch vielen einzigartigen und sich nicht wiederholenden Situationen und Vorgängen ausgesetzt, denen es trotz unvollkommener Information und unsicherem Ausgang zu entsprechen gilt. Dazu wird das Umfeld nach brauchbaren Informationen durchleuchtet, um das komplexe Umfeld zu verstehen und die zu bewältigenden Probleme meistern zu können (North, 1990).

Dieser Theorienkomplex, der aus drei Hauptströmungen besteht (der Theorie der Verfügungsrechte, der Agenturtheorie und der Transaktionskostentheorie), soll ausreichend Aufschluss über Unternehmen und die Verhaltensausrichtungen von Individuen geben. Es werden Erklärungsansätze im Bereich Organisationen und Institutionen sowie ein theoretischer Hintergrund gegeben, auf dem aufbauend es möglich scheint darzustellen, auf welche Art und Weise Transaktionen innerhalb von Unternehmen effizienter und effektiver durchgeführt werden können.

Basierend auf einer ökonomisch orientierten Theorie, die Aufschluss über Organisationen und deren Austauschbeziehungen gibt, wird ein Entwurf eines möglichen Wissensmanagement-Ansatzes elaboriert (Kapitel 6). Im Mittelpunkt steht, Unternehmen mithilfe von Wissensmanagement zu ermöglichen, das konstatierte Organisationsproblem, das ein Koordinations- und Motivationsproblem umfasst, zu über-winden. Diese Ausführungen rekurrieren auf eine Wissenskonzeption, die handlungsrelevantes Wissen als Ausgangspunkt von Wissensmanagement nimmt und in Kapitel 6.2. erarbeitet wird. Die Theorie der Neuen Institutionenö- 
konomie legt den Fokus auf die Organisationsstrukturen eines Unternehmens, die als Fundament des entwickelten Wissensmanagement-Ansatzes dienen sollen (Kapitel 6.3.). Ferner wird aufgrund der dieser Theorie zugrunde liegenden Annahme eines methodologischen Individualismus das Individuum in den Mittelpunkt der Betrachtung gelenkt (Kapitel 6.4.). „Kommunikation“ sowie "Qualität" sind zwei Aspekte, die sich im Zuge der Entwicklung des WissensmanagementAnsatzes, als besonders bedeutend herauskristallisieren und in Kapitel 6.5. sowie 6.6. näher erörtert werden. Eine Abrundung erfährt dieser Ansatz mit der Auseinandersetzung einer möglichen technologischen Untermauerung (Kapitel 6.7.). Abschließende Betrachtungen zu diesem neuen Ansatz finden sich in Kapitel 6.8 .

Die Arbeit führt in Teil IV weiterführende Gestaltungsoptionen an.

Teil $V$ fasst die wesentlichen Ergebnisse der Arbeit zusammen und nimmt eine Schlussbetrachtung der Arbeit vor.

I Problemstellung und Gang der Arbeit

1. Problemstellung

2. Gang der Arbeit

II Wissensmanagement haben oder sein?

3. Theoretische Positionierung und Grundanlage der Arbeit

4. Wissensmanagement haben

III Wissensmanagement in Anlehnung an die Neue Institutionenökonomle

5. Die Neue Institutionenökonomie

6. Ein Entwurf eines Wissensmanagement-Ansatzes

IV Weiterführende Gestaltungsoptionen

V Zusammenfassung und Ausblick 


\section{Wissensmanagement haben oder sein?}

\section{Theoretische Positionierung und Grundanlage der Arbeit}

Je nachdem, welcher paradigmatische Zugang zu einem Forschungsfeld gewählt wird bzw. von welchem theoretischen Gesichtspunkt aus eine Fragestellung behandelt wird, üben die einer Arbeit zugrunde gelegten Annahmen einen großen Einfluss auf die produzierten Ergebnisse aus.

Organisationstheorien zeichnen sich durch ihre Zugehörigkeit zu verschiedenen Paradigmen aus, wobei ein Paradigma die grundlegenden Annahmen eines Forschers über den Zweck seiner Tätigkeit (Erkenntnisinteresse), den Charakter des untersuchten Gegenstandes (Ontologie) und die geeignete Methodik zu dessen Erforschung (Epistemologie bzw. Methodologie) erfasst. Stand im antiken Griechenland noch das Wissenschaftsziel des Erkenntnisinteresses dermaßen im Vordergrund, dass von einem Erkennen um des Erkennens willen gesprochen werden kann, so rückte mit den Jahrhunderten immer mehr das Wissenschaftsziel des Erkenntnisinteresses in den Vordergrund (vgl. Schanz, 1999).

Das Feld der Betriebswirtschaft umschließt zahlreiche Organisationstheorien, deren Systematisierung oftmals versucht worden ist. Grundsätzlich dienen Organisationstheorien dem Zweck, das Entstehen, das Bestehen und die Funktionsweise von Organisationen zu erklären bzw. zu verstehen. Es wird untersucht, aus welchen Gründen und Ursachen sowohl Gemeinsamkeiten aber auch Unterschiede zwischen und in Organisationen zu beobachten sind. Systematisierende Gesamtdarstellungen der Organisationsforschung unterscheiden sich in ihren Erklärungsansätzen durch ihre unterschiedlichen Herangehensweisen. Einerseits lassen sich Unterschiede hinsichtlich der Ebene der Analyse, andererseits hinsichtlich der Rolle, die den Organisations-mitgliedern zugewiesen werden, konstatieren. Das Analyse-Ebenen-Modell umschließt Ansätze, die auf der einen Sei- 
te die Mikroebene der Organisationsmitglieder und inrer organisatorischen $\mathrm{Zu}$ sammenfassung und auf der anderen Seite im Rahmen einer Makrobetrachtung Organisationen als kleinste analytische Einheit zu ihrem theoretischen Ausgangspunkt nehmen. Erstere versuchen Organisationen vom Handeln und Verhalten der Individuen ausgehend zu erklären, wobei letztere Organisationen als Produkt bestimmter gesellschaftlich-kultureller Einflüsse verstehen. Betrachtet man andererseits den Status, der Organisationsmitgliedern und inrem Handeln zugesprochen wird, findet man Ansätze, die davon ausgehen, dass Individuen die Rolle von Entscheidungsträgern zugesprochen wird und Organisationen als Ergebnis von Wahlhandlungen erklärt werden können. Dem gegenüber stehen Konzepte, die die Begrenzungen der Entscheidungsfreiheit von Individuen durch die Bedingungen des Umfeldes hervorheben. Es zeigt sich, dass beide Dimensionen ähnliche Erklärungsmuster zugrunde legen und dies führt auch dazu, dass lediglich zwei Typen an Erklärungsmustern letztendlich zu beobachten sind: zum einen werden Organisationen rein individualistisch-reduktionistisch, zum anderen rein strukturell zu erklären versucht (vgl. Ebers, 1985).

Würde man in dieser Arbeit diese Systematisierung von Organisationstheorien mit den Ausführungen im Bereich Wissensmanagement verknüpfen, ließen sich die anfangs gestellten Fragen nicht mit Zufriedenheit beantworten. Es zeigt sich nicht von unmittelbarem Interesse, auf welcher Ebene sich Wissensmanagement darstellt, sondern vielmehr, auf welcher Annahme ein praktikabler Lösungsvorschlag für ein Wissensmanagement beruhen sollte, nämlich ob Organisationen Wissensmanagement haben oder sind. Die Bedeutung des Umfelds kann bestimmt werden, wenn ausgemacht wurde, wo Wissen gespeichert wird und wie es für Unternehmen verfügbar gemacht werden kann, sodass es managebar wird, wobei auf der Annahme aufgebaut wird, dass für ein Unternehmen relevantes Wissen innerhalb des Unternehmens selbst lokalisiert werden kann.

Ein weiterer Systematisierungsversuch von Organisationstheorien bezieht sich auf Burrell/Morgan (1979), die von der Grundthese ausgehen, dass sämtliche Organisationstheorien auf einer Erkenntnistheorie („philosophy of science") und 
einer, wenn auch oftmals impliziten, Gesellschaftstheorie ("theory of society") basieren.

Wird der Erkenntnisgegenstand oder das zu untersuchende Phänomen als "real" bezeichnet, was bedeutet, dass es auf relativ objektive Weise gemessen werden kann, und werden daraus verallgemeinernde und gesetzesähnliche Schlüsse gezogen, so stellt dies die eine Extremposition eines Gegensatzpaares, den Objektivismus, dar. Im Gegensatz dazu steht der Subjektivismus oder auch die interpretative Perspektive, deren Vertreter die Ansicht vertreten, dass die subjektiven Annahmen eines jeden Beobachters bereits den Untersuchungsgegenstand bzw. das zu analysierende Phänomen determinieren und vorweg die Entscheidung beeinflussen, auf welche Art und Weise dieses interpretiert wird.

Ordnet man nun die vier zugrunde liegenden bipolaren Problembereiche in dieses Objektiv-Subjektiv-Raster ein, so stellen sich die einzelnen Strömungen wie folgt dar. Dabei gelten der subjektive und der objektive Ansatz als dichotome Begriffe, innerhalb derer Zwischenformen auftreten können (vgl. beispielsweise Hunt, 1991).

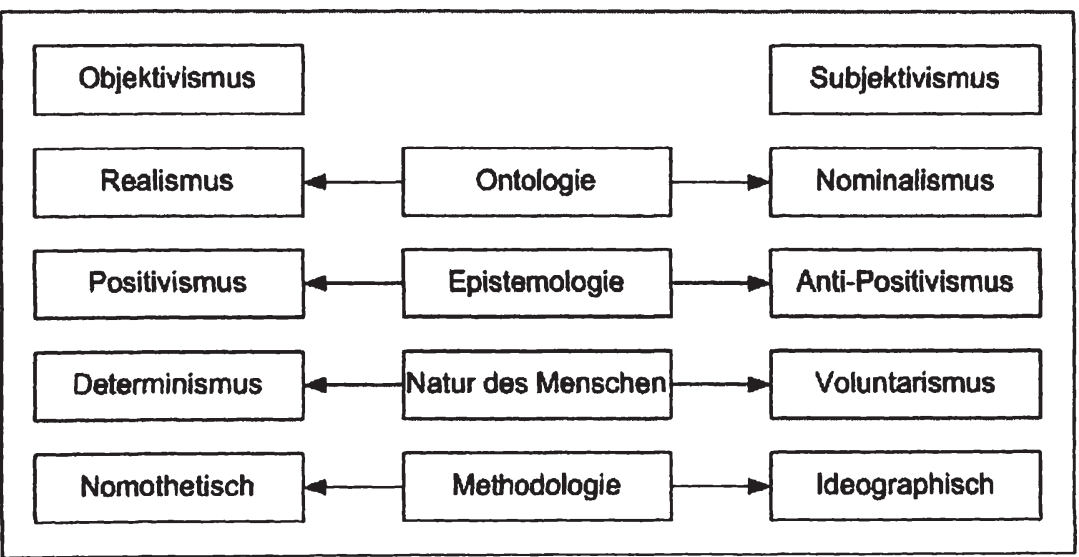

Abbildung 2: Analyseraster Objektivismus ârä Subjektivismus (Burrell/Morgan 1979) 
nehmungen eine objektive Realität, die wir uns aneignen können? Oder ist die Wirklichkeit erst das, was wir mit unseren Wahrnehmungen kreieren, und somit ein Produkt unseres eigenen Bewusstseins?

Die ontologische Ausrichtung kann wiederum in zwei den Extrempunkten zuzuordnenden Strömungen unterteilt werden: Während die Realisten die zu erforschende Realität außerhalb des Individuums sehen und sich diese Wirklichkeit von außen in dem individuellen Bewusstsein widerspiegelt, erachten die Nominalisten die Realität als ein Produkt individuellen Bewusstseins.

Eng verbunden mit der Betrachtung der Wirklichkeit ist die Frage, wie wir die Welt zu verstehen beginnen und dieses Wissen anderen kommunizieren. Wie kann Wissen ergründet werden? Welche Formen von Wissen gibt es? Kann man die Dichotomie „richtig" und "falsch" rational argumentieren und nach objektiven Kriterien erklären? Derartige Fragestellungen sind Gegenstand epistemologischer Auseinandersetzungen mit der Wirklichkeit. Auch hier werden zwei Strömungen an den Endpunkten des Forschungskontinuums ausgemacht: der Positivismus wie der Anti-Positivismus. Vertreter der ersten Richtung gehen von einer objektiven Wirklichkeit aus und sehen Individuen als fähig an, das, was sich „außen“ darstellt, erfassen und wahrnehmen zu können und ein Bild davon zu gestalten, was in dieser Wirklichkeit als richtig und als falsch verstanden werden soll. Die zweite Strömung, die in ihren extremsten Ansätzen eine objektive Realität gänzlich verneint, leugnet folglich auch eine Wissenschaft, die objektives Wissen zu generieren behauptet.

Der dritte Problembereich scheint konzeptionell von den beiden ersten Analysebereichen abgeschnitten zu sein, dennoch werden auch die auf die Natur des Menschen bezogenen Annahmen mit der ontologischen und epistemologischen Dimension verbunden. Die Beziehung des Menschen zu seiner Umwelt stellt eine Relation dar, die als Untersuchungsobjekt essentiell ist. Auf welche Art und Weise reagieren Individuen auf Vorkommnisse in ihrer Umwelt? Oder werden Vorkommnisse in der Umwelt durch das Agieren von Individuen verursacht? Werden 
Erfahrungen durch den Menschen selbst gemacht oder sind sie letztendlich "nur" ein Produkt des Umfelds? Vertreter des Determinismus sprechen von einer Kausalität zwischen den auslösenden Faktoren in der Umwelt und dem daraus resultierenden Verhaltensmuster. Externe Situationen determinieren das Reaktionsmuster eines Individuums. Gänzlich konträr dazu ist der voluntaristische Zugang zu der Natur des Menschen, der von einer Handlungsfreiheit des Menschen ausgeht. Die Umwelt wird als Kreation des Menschen selbst angesehen.

Wie gelangen wir nun zu den Erkenntnissen über unsere Umwelt oder uns selbst? Die Art und Weise, wie Wissen erlangt wird, auch Methodologie genannt, wird stark durch das obige Dreierpaket an Annahmen beeinflusst. Das durch Burrell/ Morgan (1979) festgesetzte Gegensatzpaar erschließt sich einerseits in einem nomothetischen Zugang und andererseits in einem ideographischen. Ersterer ist eng mit den Forschungsmethoden der Naturwissenschaften verbunden. Werden soziale Phänomene und die soziale Welt als extern zum Individuum gesehen, die als harte und reale Gegebenheiten nach objektiven Kriterien abgebildet werden können, so wird sich der Zweck der Forschung darin begründen, diese objektiven, harten und realen Elemente zu identifizieren und zu definieren, um Gesetzmäßigkeiten zu entdecken und allgemein gültige Aussagen zu treffen. Der ideographische Zugang hingegen basiert auf den Annahmen von Vertretern, die die Welt mit weitaus "weicheren“ Termini beschreiben und den sozialen Phänomenen eine persönliche und subjektive Qualität zusprechen. In den Vordergrund rücken vielmehr das Verstehen an sich und das menschliche Handeln, die Konstruktion und Interpretation einer Wirklichkeit sowie das fortwährende Modifizieren der Handlungs- und Denkstrukturen werden zum Gegenstand von Analysen. Allgemeine Gesetzmäßigkeiten werden nicht festzustellen versucht bzw. in den extremsten Ansätzen der interpretativen Perspektiven gänzlich angezweifelt. Somit gilt es, sich mit einzigartigen und besonderen Merkmalen auseinander zu setzen, die bei individuellen Handlungsmustern auftreten.

Die zweite Dimension bildet ein weiteres Forschungskontinuum, dem die Annahmen über die Natur der Gesellschaft zugrunde liegen. Einerseits versteht sich
Saran Meisenberger - $978-3-631-75453-5$ 
Die zweite Dimension bildet ein weiteres Forschungskontinuum, dem die Annahmen über die Natur der Gesellschaft zugrunde liegen. Einerseits versteht sich die Soziologie des radikalen Wandels als eine Disziplin, die vor allem Veränderungen, strukturelle Konflikte, Herrschaft, Widersprüche und Emanzipation in den Vordergrund stellt. Die Soziologie der Regulation, das andere Ende des Kontinuums, legt ihre Aufmerksamkeit vorwiegend auf Fragen des Status quo, der Ordnung, des Konsens, der Integration und Solidarität. Je nach gewählter methodischer Grundausrichtung kommt es in den einzelnen Theorien zu einer Schwerpunktsetzung auf unterschiedliche Fragestellungen und Problembereiche. Die Zusammenführung dieser Dichotomien mit den vorangegangenen bipolaren Zuweisungen der methodischen Grundannahmen stellt vier Paradigmen zur Diskussion, die voneinander unabhängig, jedoch in sich kohärent, als Basis für die Entwicklung von Organisationstheorien dienen.

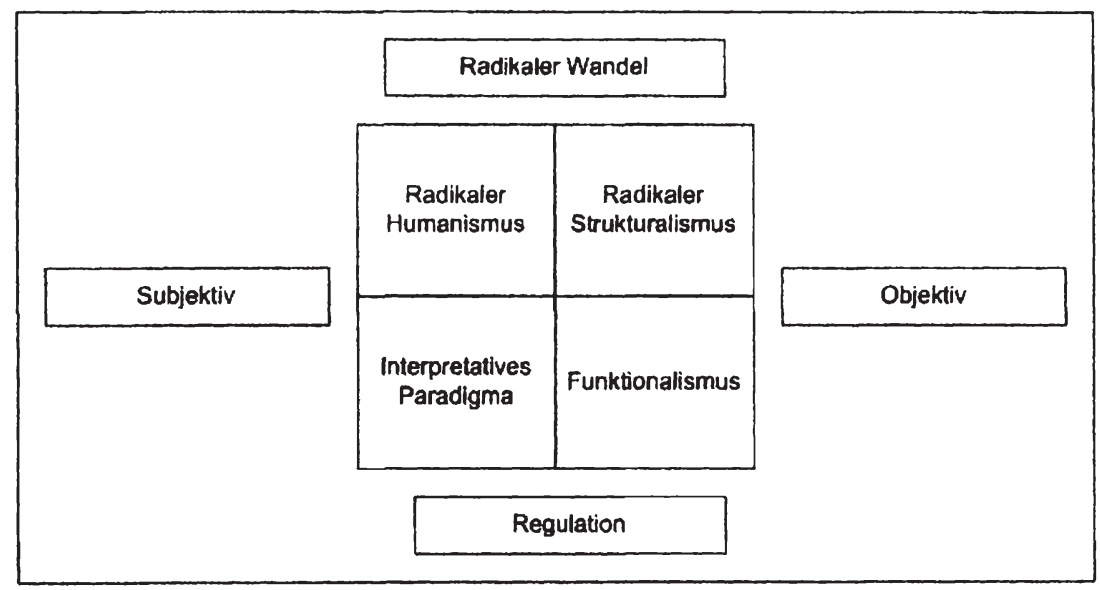

\section{Abbildung 3: Paradigmen (Burrell/Morgan, 1979)}

Beginnend mit den Paradigmen, die auf den Zielsetzungen basieren, den Status quo sozialer Ordnung zu kritisieren und zu verändern, kommt es einerseits zu einer Anlehnung an den Subjektivismus in Form des Radikalen Humanismus und 
am sozialen Wandel, wobei unterschiedliche und voneinander abzugrenzende Zugänge zur Analyse desselbigen gewählt werden. Der Radikale Humanismus sieht seine Legitimation darin begründet, die Mitglieder sozialer Einheiten von Bevormundung, Entfremdung, Ausbeutung und Unterdrückung zu befreien. Ausgehend davon, dass die bestehenden Strukturen und Machtkonstellationen Konstruktionen der Akteure selbst sind, stellt die Kritik an Machtprozessen den grundlegenden Forschungsschwerpunkt dar. Die subjektiven Sinngehalte der Akteure betrachtend, untersucht hingegen der Radikale Strukturalismus auf objektiven Annahmen basierend die sozialen Strukturen der Welt. Fundamentale Konflikte lassen sich als dort verwurzelt und bestehend begründen. Somit gilt es die sozialen Strukturen als Kern jeglicher sozialen Unordnung zu verstehen, zu erklären und zu kritisieren, um deren Wandel hervorzurufen.

Das funktionalistische und das interpretative Paradigma stehen beiden ersteren Paradigmen bipolar gegenüber, wobei der Funktionalismus die dominierende Strömung in den Sozial- und Wirtschaftswissenschaften darstellt. Zahireiche Organisationstheorien fußen auf diesem Paradigma, indem sie soziale Systeme als reale Entitäten betrachten, die von äußeren Kräften erhalten werden und die unabhängig von ihren Mitgliedern wirken. Es werden soziale Regelmäßigkeiten zu finden und der soziale Status quo zu erfassen versucht, um mittels einer deduktiven Herangehensweise kausale Zusammenhänge aufzustellen und generalisierte Aussagen zu treffen, die den Anspruch auf Allgemeingültigkeit erheben.

Vertreter des interpretativen Paradigmas hingegen sehen soziale Realitäten als soziale Konstrukte, die durch das Handeln der Akteure zustande kommen. Die subjektiven Sinngehalte der Akteure unterliegen einer Analyse. Trotz der grundsätzlich divergierenden methodischen Grundausrichtung beider letzteren Paradigmen ist ihnen gemeinsam, dass sie sich nicht mit dem Problem sozialer Konflikte und der Legitimierung sozialen Wandels auseinandersetzen, sondern stets die Analyse des Status quo sozialer Ordnung als Gegenstand haben. 
Das Konzept von Burrell/Morgan war Gegenstand zahlreicher kritischer Diskussionen, in denen die Systematisierung der verschiedenen Paradigmen thematisiert wurde. Insbesondere rund um die Kompatibilität bzw. Inkompatibilität der vier Paradigmen entfachte die sog. "Inkommensurabilitätsdiskussion" (Morgan, 1986; Goia/Pitre, 1990; Scherer, 1998; Kieser, 1999): Paradigmen als Standards der Wissenschaftlichkeit werden innerhalb einer Disziplin anerkannt und außerhalb bezweifelt. Somit wird eine transparadigmatische Analyse einzelner Zugänge verneint (Kuhn, 1970). Eine Überwindung dieser Kritikpunkte und der Widersprüche und Differenzen zwischen den einzelnen divergierenden Paradigmen scheint nur begrenzt möglich, auch wenn es Theorien gibt, die genau diese Dichotomien zu überwinden trachten ( $v g l$. die Systemtheorie von beispielsweise Luhmann oder die von Giddens begründete Strukturationstheorie).

Die von Burrel//Morgan (1979) festgesetzte Dichotomie mit seinen Extrempunkten des Objektivismus und Subjektivismus spiegelt nicht alte Diskurse wie die zwischen den Rationalisten und Empiristen oder den Positivisten und den Kritischen Rationalisten wider, ganz im Gegenteil. So öffnet sich bereits bei dieser ersten Darstellung des Analyserasters mit der Gliederung in vier Bereiche (Ontologie, Epistemologie, Methodologie und die Natur des Menschen) ein weites Feld an Kritikpotential. Zu starr und zu undurchsichtig werden die Grenzen der einzelnen Bereiche gezeichnet. Oftmals wurde diesem Schema der Mantel eines Objektiv-Subjektiv-Kontinuums übergezogen, um die in der Literatur kaum wiederfindbaren Extremgruppen den Organisationstheorien doch zugänglich zu machen und eine Systematisierung derselbigen zu ermöglichen (vgl. beispielsweise Hunt, 1991; Kieser, 1999).

Die betriebswirtschaftliche Literatur im Bereich Wissensmanagement ist insbesondere durch die Paradigmen des Funktionalismus sowie des interpretativen Paradigmas geprägt, weshalb die Strömungen des Radikalen Humanismus sowie des Radikalen Strukturalismus in dieser Arbeit nicht diskutiert werden. 
Unterschiedliche Paradigmen basieren auf unterschiedlichen Wissenskonzepten, die wiederum eigene Herangehensweisen an den Bereich Wissensmanagement intendieren. So findet sich in einem funktionalistischen Paradigma Wissensmanagement als ein funktional-instrumentelles Konzept der rationalen Unternehmensführung. Es basiert auf einem Wissenskonzept, das von einer realen und objektiv abbildbaren Wirklichkeit ausgeht, die Individuen zugänglich ist. Wissensmanagement, das im Rahmen eines interpretativen Paradigmas entwickelt wird, wird als systemimmanentes Phänomen einer sozialen Praktik verstanden und im Rahmen des jeweilig sozialen Kontextes rekonstruiert. Interpretative Theorien basieren eher auf einem Wissenskonzept des "contextualism" (Tsoukas, 1996), das gegenwärtige Ereignisse vor allem in der Vergangenheit verwurzelt sieht.

Eine wissenschaftliche Arbeit erfordert eine wissenschafts-theoretische Positionierung. Das Framework nach Burrell/ Morgan (1979) fand in der betriebswirtschaftlichen Literatur eine große Resonanz, obwohl die Dichotomie des Objektivismus und Subjektivismus in der präsentierten Form kaum vorzufinden ist. Sie ist eher durch ein Kontinuum von unterschiedlichen Ansätzen mit bestimmten Abstufungen gekennzeichnet (Morgan/Smirchich, 1980). Autoren der sozial- und wirtschaftswissenschaftlichen Disziplin, die sich in dieser Arbeit als namhafte Vertreter und Forscher im Bereich Wissensmanagement wiederfinden, nehmen selten, wenn überhaupt, zu ihren epistemologischen, ontologischen oder methodologischen Grundausrichtungen Stellung. Eine Zuordnung der unterschiedlichen Ansätze nach dieser Systematisierung wird daher nicht erfolgen, da Ansätze im Forschungsbereich des Wissensmanagements oftmals Grundannahmen beider Forschungsparadigmen kombinieren bzw. in den einzelnen Dimensionen mal zum Objektivismus mal zum Subjektivismus tendieren.

Von Interesse zeigt sich, wo die dargestellten Ansätze Wissen lokalisieren und welche Problemfelder bzw. Fragestellungen sie mit Wissensmanagement zu regeln bzw. zu beantworten versuchen. Welche Rolle spielen Individuen im Zusammenhang mit Wissensmanagement? Welche Lösungsvorschläge werden geboSarah Meisenberger - 978-3-631-75453-5 
ten, Wissen der Mitarbeiter dem Unternehmen besser zugänglich zu machen, indem individuelles Wissen beispielsweise auf organisationale Ebene transferiert wird und Unternehmen darin unterstützen, einen Wettbewerbsvorteil gegenüber ihren Mitbewerbern zu erlangen?

Fragestellungen dieser Art greifen auf Grundannahmen eines funktionalistisch geprägten Paradigmas zurück. Die Frage, ob Unternehmen Wissensmanagement haben oder sind, spiegelt die Dichotomie des Objektivismus und Subjektivismus wider. Genauso wenig wie einzelne Autoren einer der beiden Logiken zugeordnet werden können, lässt sich diese Frage eindeutig beantworten. Die vorliegende Arbeit wird die Probleme und Defizite von Wissensmanagement aus einem funktionalistischen Paradigma beleuchten und stellt sie - an Stellen, wo dies als angebracht erwogen wird - Ideen eines interpretativen Paradigmas gegenüber.

In der Folge wird Wissensmanagement in seine Einzelteile (Teilbereich Wissen, Management und Wissensmanagement als Gesamtes) gegliedert. Um einen runden Überblick über diese Forschungsbereiche zu gewährleisten, wird es in diesen drei Kapiteln als notwendig erachtet, Grundannahmen und theoretische Ausführungen beider Logiken (funktionalistisch und interpretativ) darzustellen.

Die in der betriebswirtschaftlichen Wissensmanagementliteratur gängigsten Wissensmanagement-Ansätze eines funktionalistischen Paradigmas werden daran anschließend diskutiert. Die dargestellten Ansätze wurden nach Studium der betriebs-wirtschaftlichen Literatur im Bereich Wissensmanagement nach ihrer Popularität ausgewählt. 


\subsection{Wissen - DIE Ressource oder Resultat von Interaktion?}

Wissen wird oftmals als die Schlüsselressource und als der komparative Vorteil eines Unternehmens gegenüber seinen Mitbewerbern gesehen (Drucker, 1992; Prusak, 1997; Hamel/Prahalad, 1994; Probst/Büchel/Raub, 1998). Daher richten mehrere Autoren ihren Schwerpunkt darauf, diese Ressource auszumachen und unterstreichen die Notwendigkeit, Wissen dem Unternehmen zugänglich und managebar zu machen (Probst et al, 2000). Andere hingegen definieren Wissen wiederum als Firmenvermögenswert (Davenport/Prusak, 1998) oder als Kernkompetenz, die implizites wie explizites Wissen subsumiert und als ein Mix an Technologie und Skills gewertet werden kann (Prahalad/Hamel, 1990; LeonardBarton, 1995; Hamel/Prahalad, 1994).

Wird Wissen im Rahmen eines funktionalistischen Paradigmas betrachtet, bedingt dies, dass es gemäß einer realistischen Ontologie als etwas Wirkliches und a priori Existierendes angesehen wird. Wissen, das die Wirklichkeit reflektiert, kann als Objekt zugänglich gemacht werden. Oftmals in dieser Logik anzutreffen ist eine Trennung zwischen den Termini "Wissen", "Daten" und „Informationen“. Diese drei Begriffe führen immer wieder zu Missverständnissen bzw. sorgen für Irrtümer, da sie unsystematisiert und häufig synonym verwendet werden. Um die Begriffsdefinitionen der einzelnen Termini auseinander zuhalten, wurden sie von Rehäuser/Krcmar (1996) in einer Begriffshierarchie zusammengefasst, was auf eine große Resonanz in der betriebswirtschaftlichen Literatur gestoßen ist. 


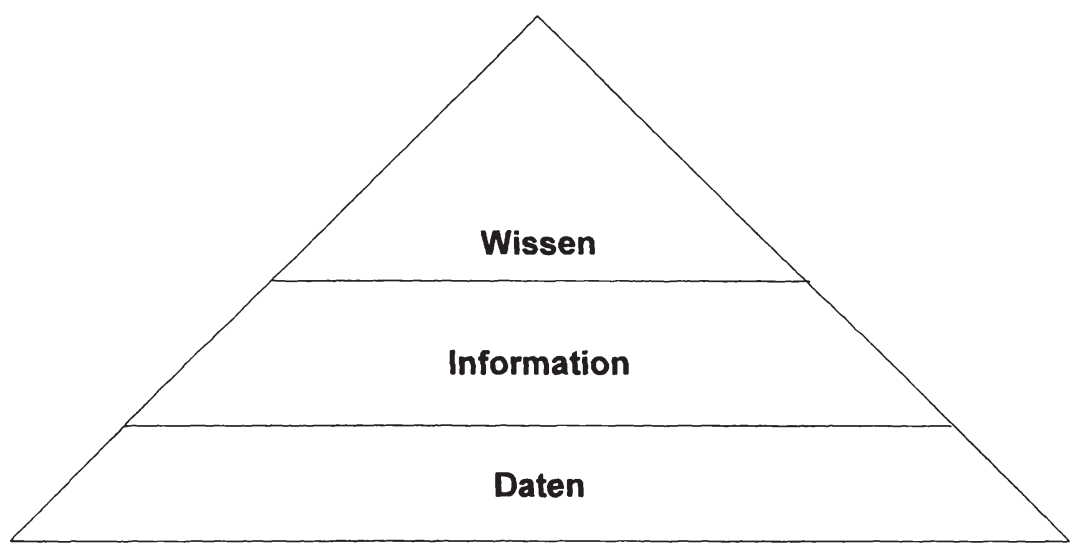

Abbildung 4: Begriffspyramide Daten - Informationen - Wissen (Rehäuser/Krcmar, 1996)

Beginnend mit dem Begriff "Daten" ist dies das Fundament, auf dem Erkenntnis aufbauen soll und mit dem Erfahrung gemacht wird. Auch wenn man untersuchen kann, aus welchen Teilmengen sich Daten zusammensetzen, so greift man dabei schon weit in das Forschungsgebiet der Informatikwissenschaften hinein, was in dieser Arbeit als nicht zielführend erachtet wird. Es soll lediglich festgehalten werden, dass Daten Syntaxregeln folgend durch Zeichen gebildet werden (Rehäuser/Krcmar, 1996). Somit können Daten als Unterlage bzw. Grundlage für die Lösung einer Aufgabe gesehen werden, die objektive Fakten zu Ereignissen oder Vorgängen bezeichnen. Sämtliche Angaben über Sachverhalte und Dinge, sei es in gedruckter, gespeicherter, visueller, akustischer oder sonstiger Form, können unter dem Begriff „Daten" subsumiert werden. Es ist möglich, Daten objektiv wahrzunehmen und potentiell zu verwerten (Davenport/Prusak, 1998). Mit dem Ansammeln von Daten ist jedoch noch nicht gewährleistet, dass dies auch in deren Verwendung resultiert. Denn bevor die objektiv festgestellten Sachverhalte in Verwertbares konvertiert werden können, müssen sie von einem Individuum in Beziehung zu dessen Realitätsumgebung gesetzt werden. Durch diesen Prozess entstehen Informationen, die als diejenigen Daten definiert werden können, die von Individuen subjektiv wahrgenommen und persönlich verwertet werden. Sie stellen für den einzelnen Empfänger relevantegAussagen5dab-die sich 
auf einer höheren Abstraktionsebene befinden als Daten, da letztere die geschlossene Einheit von Informationen bilden. Wissen entsteht in diesem Zusammenhang erst dann, wenn Informationen in bestimmte Erfahrungskontexte eingebunden werden (Güldenberg, 1997).

Auf welche Art und Weise die verschiedensten Formen von Wissen systematisiert werden können, wird in diversen Wissenskategorisierungen festzuhalten versucht (eine Zusammenfassung dieser findet sich bspw. bei vonKrogh/ Roos/ Kleine (1998), Neumann (2000), Blackler (1995) oder Lam (2000)).

Eine Gemeinsamkeit der Wissensklassifizierungen wird in der betriebswirtschaftlichen Literatur auf der Ebene der Epistemologie sowie der Ontologie festgestellt. Epistemologisch betrachtet kann Wissen einerseits explizit artikuliert werden oder sich implizit manifestieren (Polanyi, 1962). Der Schwerpunkt wird meist auf das explizite, das heißt auf das beschreibbare, standardisierbare Wissen, das methodisch und systematisch in Systemen, Strukturen, Prozessen, Dokumentationen, Datenbanken etc. gespeichert werden kann, gelegt. Dieses Wissen kann außerhalb von Individuen in formaler Sprache festgehalten und unabhängig vom Wissenden selbst verbreitet, übertragen und gespeichert werden, daher auch der Name "disembodied knowledge“. Es ist zeitlich stabil, da es allgemein verfügbar ist (vgl. Blackler, 1995).

Implizites Wissen hingegen ist tief in den Handlungen und Erfahrungen von Individuen verankert und kann daher auch nicht von Individuen getrennt werden ("embodied knowledge", dieser Ausdruck wurde geprägt von bspw. Zuboff, 1988; Blackler, 1995; Nonaka/Takeuchi, 1995; Collins, 1993). Implizites Wissen ist lediglich schwer formalisierbar, kommunizierbar und teilbar und zeigt sich mit intuitivem Charakter. Wenn es transferiert wird, dann erfolgt dies gekoppelt an eine Handlung und erfordert eine enge Interaktion von Individuen, die auf einem gemeinsamen Werteverständnis aufbauen (vgl. Lam, 2000). 
Wie Polanyi (1962) in seinen Ausführungen feststellte, basiert ein Großteil von Entscheidungen auf implizitem Wissen, das sich in Werten, Symbolen, Metaphern usw. ausdrückt. Nicht zuletzt deshalb spielt das implizite Wissen in Organisationen eine tragende Rolle. Handlungen werden durch das implizite Wissen gesteuert und Individuen gelangen dadurch zu ihren Resultaten.

Die ontologische Dimension gibt Aufschluss darüber, wo Wissen lokalisiert werden kann. Einerseits ist das Individuum selbst Träger von Wissen, das in den verschiedensten Situationen sowie bei Frage- und Problemstellungen Anwendung findet. Andererseits manifestiert sich Wissen auch auf organisationaler Ebene, sobald Individuen miteinander interagieren und Wissen kollektiv geteilt wird. Es ist in den personenunabhängigen, anonymisierten Regelsystemen, Standardverfahren, Leitlinien, Kodifizierungen etc. eingebettet und umfasst Wertvorstellungen, Erinnerungen an vergangene Ereignisse, Zielsetzungen usw. (Zander/Kogut, 1995). Diese Betrachtungsweise setzt voraus, dass Organisationen als soziale Entitäten akzeptiert werden, die selbst Wissen erwerben und eine eigene wissensbasierte Identität entwickeln können (vgl. Brown/Duguid, 1991; Weick/Roberts, 1993; Nelson/Winter, 1982). Demgegenüber steht eine kritische Haltung von bspw. Simon (1991), der davon ausgeht, dass Organisationen nicht per se über Wissen verfügen können, sondern sich organisationales Wissen ausschließlich durch die Aufsummierung von individuellem Wissen entwickelt.

Aus diesen zwei Dimensionen (Epistemologie und Ontologie) und ihren jeweils zwei Ausprägungen lassen sich vier Arten von Wissen kategorisieren (Blackler, 1995 adaptiert von Collins, 1993): „embrained knowledge“, „embodied knowledge“, „encoded knowledge" sowie „embedded knowledge“:

- „Embrained knowledge" (vgl. Blackler, 1995; Argyris/Schön, 1978; Fiol/Lyles, 1985), das sich auf der individuellen Ebene ausdrückt, ist Wissen von den harten Fakten wie Naturgesetzen usw. Dieses Wissen beeinflusst die Fertigkeiten und Fähigkeiten von Individuen. Es ist formal, abstrakt und in theoretischer Form vorhanden. Mit diesem Wissen sind Individuen befähigt, zugrun- 
de liegende Muster von etwas (wie bspw. von einer neuen Branche) zu erkennen bzw. zugrunde liegende Annahmen zu reflektieren (vgl. „double-looplearning" bei Argyris/Schön, 1978).

- „Embodied knowledge" (Zuboff, 1988; Blackler, 1995; Nonaka/Takeuchi, 1995) generiert sich über Handlungen und steht einem Individuum implizit durch sein Tun und seine praktischen Erfahrungen zur Verfügung.

- "Encoded knowledge" (Zuboff, 1988; Blackler, 1995) wird oftmals mit "Informationen" gleichgesetzt, die durch Zeichen und Symbole vermittelt werden. Es ist kollektiv und explizit vorhandenes Wissen, das in Plänen, Verfahrensweisen, Richtlinien usw. kodifiziert ist. Es hat einen richtungweisenden Charakter, der den Output eines Unternehmens steuern hilft.

- „Embedded knowledge“ (Berger/Luckmann, 1999; Brown/Duguid, 1991) stellt die kollektive Form von implizitem Wissen dar, das in Organisationsroutinen und in geteilten Werte- sowie Normvorstellungen verankert ist. Es hat dynamischen und organischen Charakter, der in Abwesenheit von geschriebenen Regeln komplexe Interaktionsstrukturen innerhalb eines Unternehmens unterstützt.

Die funktionalistische Forschungstradition wird mittels eines interpretativen Paradigmas überwunden, indem es zu einer konstruktivistischen Betrachtung von Wissen kommt. Wissen wird durch das Handeln von Individuen gebildet und geformt. Es liegt eine breite Begriffsdefinition von Wissen zugrunde und Forschungsbeiträge in diesem Paradigma betonen vor allem die soziale Natur der Wissensgenerierung. Starke Überschneidungen können an dieser Stelle mit der Begriffsdefinition des impliziten Wissens (nach Polanyi, 1962) festgestellt werden. Dominierend gestalten sich in einer interpretativen Logik Ähnlichkeiten mit Modellen des Organisationalen Lernens. So wird der Prozess der Wissensteilung vor allem als Beziehung zwischen Handlungen, Verstehen und Kommunikation verstanden. Jedoch auch hier lassen sich Modelle finden, die stark im Lichte ei- 
nes funktionalistisch geprägten Forschungszugangs entwickelt worden sind und auf eine präskriptive oder präskriptiv orientierte deskriptive Weise Aussagen über die Wirklichkeit treffen (vgl. beispielsweise Senge, 1991; Argyris/Schön, 1978; Duncan/Weiss, 1979). Es werden jedoch keine Lösungen für "reale" Probleme angestrebt, da durch die soziale Konstruktion der Wirklichkeit die Legitimität dafür entzogen wird (Schultze, 1999).

Wissen als Prozess oder Praktik, die vom Handeln nicht zu trennen ist (vgl. Cook Brown, 1999), wurde aus den "action-based theories" (auf den russischen Psychologen Vygotsky zurückgehend) abgeleitet (vgl. nähere Auseinandersetzungen mit dieser Theorie beispielsweise bei Engeström, 2000; Blackler, 1995; Brown/ Collins/Duguid, 1989; Lave/Wenger, 1991). Diesen Annahmen liegen Ideen von beispielsweise Hegel und Kant wie auch die Theorie des dialektischen Materialismus zurückgehend auf Marx und Engels zu Grunde.

Die "Activity Theory" entstand in einer Zeit, in der psychologische Theorien auf einer Reflexion des Verhaltens (Stimulus-Response-Beziehung) basierten und in der das Bewusstsein des Menschen als Stimulus für das menschliche Verhalten fungierte. Dennoch vermochte die "Activity Theory", den Ansatz der StimulusResponse-Beziehung umzudrehen und als neuartiger Beitrag in der Psychologie wurde das Prinzip der Einheit und Untrennbarkeit des Bewusstseins (des menschlichen Verstandes) und des Handelns vorgetragen. So konstituiert nicht mehr das Bewusstsein des Menschen dessen Handlungen, sondern soziale Erfahrungen formen das menschliche Bewusstsein. Diese Erfahrungen sind in einen Kontext gebettet, der durch die Interaktion von Individuen eine Bedeutung erhält. Eine relationale Verbindung mit der sozialen und physischen Welt wird hergestellt (vgl. Cook/Brown, 1999).

Um Handlungen durchzuführen, muss ein Ziel bestehen, das man durch die Handlungen zu erreichen versucht. Dabei sind Menschen eingebettet in eine Wirklichkeit, die aus objektiv konstituierenden Gegenständen im weitesten Sinne (alles, was durch die Naturwissenschaften als objektiv gegeben akzeptiert wird), 
jedoch auch aus sozial und kulturell determinierten "Gegenständen" (unter dem Prinzip der "object-orientedness" zusammengefasst) besteht. In welcher Form ein Objekt wahrgenommen wird, zeichnet sich abhängig von dem Kontext, innerhalb dessen Interaktionen mit diesem Objekt stattfinden. Wissen über die „reale" Welt wird durch Interaktionen mit der Welt entwickelt. Interaktionen zwischen Individuen und der Welt werden funktional in Form von Hierarchielevels organisiert, die in Form von "activities", „actions" und "operations" zum Ausdruck kommen. Somit bleiben zwar die Objekte der Wirklichkeit fix, jedoch die Ziele und Aktivitäten unterliegen einer Veränderung, was gleichzeitig den flexiblen Aspekt der „Activity Theory" determiniert (vgl. Engeström, 2000).

Aktivitäten weisen eine interne sowie externe Seite auf. Die traditionelle Idee mentaler Prozesse umfasst die internen Gegebenheiten, wobei diese nur dann verstanden werden können, wenn sie nicht getrennt von der externen Seite analysiert werden. Mentale Prozesse manifestieren sich in externen Aktivitäten, die von der Person ausgeführt werden. Der generelle Kontext einer Aktivität (der eben beide - interne sowie externe - Komponenten inkludiert) bestimmt, wann und warum externe Aktivitäten internalisiert werden und vice versa. Wie menschliche Aktivität überhaupt zustande kommt, kann durch das Prinzip der "mediation" erklärt werden. So werden Handlungen sozial mediiert, was bedeutet, dass das Bewusstsein und die Bedeutungen von Sachverhalten bzw. Begriffen immer gemeinsam geformt werden und es sich um einen kollektiven Prozess handelt. Mittels Artefakten wird die Mediation durchgeführt, die sich - breit definiert - aus diversen Instrumenten, Zeichen, Sprachen und Maschinen zusammensetzen. Diese führen eine besondere Kultur und Geschichte mit sich und sind in den Strukturen, die sich in den Aktivitäten über Zeit und Raum manifestieren, verankert (vgl. Leont'ev, 1978; vgl. Engestrom, 1987; 1991).

Basierend auf der "Activity Theory" wurden Annäherungen zu den Konzepten des "organisationalen Lernens" und von "Wissen" vorgenommen (vgl. beispielsweise Blackler, 1995; Cook/Brown, 1999). Die einzelnen Teilströmungen ("Wissen“ substantivisch gebraucht, "wissen" als Verb und organisationales Lernen) wurden 
jedoch voneinander abzugrenzen versucht. Im Zuge des Konzepts des "knowing" ist man imstande, das gefestigte Bild zu überwinden, das oftmals mit dem Begriff "Wissen" assoziiert wird, nämlich die Betrachtung von Wissen als statische und zeitlose "Ware", die Individuen für ihr Unternehmen enwerben und besitzen können. "Lernen" wird von "wissen" insofern inhaltlich getrennt, als es als die passive Absorption von Wissen betrachtet wird. Hingegen drückt "knowing" die aktive soziale Konstruktion von Wissen aus, was eingebettet in der "infrastructure of knowing" erfolgt, die sich wiederum aus den kulturell in einem System zur Verfügung stehenden Ressourcen zusammensetzt (vgl. Blackler/Crump/ McDonald, 1999).

Wissen ist fortwährend Gegenstand von sozialen Verhandlungsprozessen. Es ist evolutionär und fest in den Lernprozessen verankert (Blosch, 2001). Der Fokus des Lernens in den Köpfen von Individuen wird überwunden, indem Lernen als Wissensgenerierung, -enwerb und -transfer angesehen wird, das nur durch die aktive Teilnahme an kollektiven sozialen Praktiken vollzogen werden kann ( $\mathrm{vgl}$. beispielsweise Cook/Brown, 1999; Kogut/Zander, 1996; Spender, 1996; Brown/ Duguid, 1991; Tsoukas, 1996): "the locus of the agent's knowing is not in his head but in practice, that is to say, his understanding is implicit in the activity in which he engages" (Tsoukas, 1996, p. 16). Auf diese Weise kann die Dualität von Tun und Handeln überwunden werden, indem die konzeptionelle Trennung unterschiedlicher Prozesse, beispielsweise "Denken", „Lernen", „Handeln", aufgehoben wird.

"Knowing" stellt in einer interpretativen Logik einen relationalen Prozess dar, wobei Wissen fortwährend entsteht. Hierbei werden vor allem das System selbst und die dem System inhärenten Dynamiken einer näheren Betrachtung unterzogen, da Wissen nur abhängig von den stattindenden Handlungen konzipiert werden kann. Somit wird "knowing" als ein Phänomen analysiert,

"which is (a) manifest in systems of language, technology, collaboration and control (i.e. it is mediated); (b) located in time and space and specific to particular Sarah Meisenberger - 978-3-631-75453-5 
contexts (i.e. it is situated); (c) constructed and constantly developing (i.e. it is provisional); and (d) purposive and object-oriented (i.e. it is pragmatic)" (Blackler, 1995, S. 1039).

Da die Aktivitätssysteme einer Organisation einer Veränderung unterliegen, sei es durch neue Entwicklungen beispielsweise auf dem Sektor der Informationsoder Kommunikationstechnologien oder sei es durch die Weiterentwicklung von Managementtechnologien, kann Wissen als nicht statisch angesehen werden, zumal es sich von den Handlungen in diesen Aktivitätssystemen abhängig zeichnet und folglich ebenfalls fortwährend revidiert werden muss (was durch die Eigenschaften "mediated" und "provisional" ausgedrückt wird). Um herauskristallisieren zu können, welches "knowing" in den jeweiligen Systemen bei den involvierten Akteuren vorherrschend ist, müssen der Kontext und die daraus resultierten Interpretationsschemata analysiert werden, wodurch das "situated knowing" erschlossen werden kann. Geht man diesen Interpretationsschemata auf den Grund, so führten sie zu den in einem Aktivitätssystem grundlegend vorherrschenden Betrachtungen, die die Akteure über ihre Handlungen und die davon berührten Objekte anstellen. Darin kommt das „pragmatic knowledge“ zum Ausdruck. Die Ausprägung dieser vier erwähnten Eigenschaften beeinflussend muss schließlich noch ein zusätzliches Charakteristikum des "knowing" genannt werden, nämlich das des "contested knowing". Hierbei wird darauf Bedacht genommen, dass Interpretationen über die Welt und die Systeme, in denen man sich befindet, stark davon abhängig sind, welche Machtstrukturen in diesem Gefüge vorherrschen und die Sicht der Dinge beeinflussen (vgl. Blackler, 1995).

Konzentriert man sich ausschließlich auf die Verankerung des Handelns im Wissen, so führt dies oftmals dazu, dass Wissen als ein Sammelbecken konzipiert wird, das alle nur erdenklichen menschlichen Fähig- und Fertigkeiten, Emotionen, Normen usw. einschließt, um nicht vorweg Ursachen für einen Handlungserfolg auszuklammern. Jede erfolgreiche Handlung ist Ausdruck eines spezifischen Wissens des Handelnden und kann somit auch nur aus dieser Einzelhandlung 
erschlossen werden. Das Wissen kann dabei bewusst, jedoch auch unbewusst in die Handlungen einfließen (vgl. Schreyögg/ Geiger, 2003).

Wissen gibt das Rahmengerüst vor, innerhalb dessen Interpretationsprozesse ausgeführt werden, und beeinflusst dadurch die Interaktionen mit der Welt und die daraus durch die Akteure entwickelten Bedeutungsschemata. Gleichzeitig dazu kann Wissen nicht als alles einschließend auftreten, da eben etwas davon im „knowing" selbst zu finden ist. Wissen an sich erhält dadurch eine andere Bedeutung und wird als Tool gesehen, das "wissen" ermöglichen hilft (vgl. Cook/Brown, 1999). „Knowing is to interact with and honor the world using knowledge as a tool" (Cook/Brown, 1999, S. 389).

Berger/Luckmann (1999) haben die Forderung erhoben, dass sich die Wissenssoziologie eigentlich mit allem beschäftigen sollte, was in der Gesellschaft weithin als Wissen gilt. Dies hat dazu geführt, dass Versuche gestartet worden sind, in Begriffsdefinitionen von Wissen auch das Alltags- sowie Allerweltswissen zu integrieren (vgl. beispielsweise Pautzke, 1989 oder Güldenberg, 1998), was vor allem durch das Konzept des "knowing" noch weiter forciert worden ist. Dadurch erhält der Wissensbegriff eine Offenheit und verliert an Konturen. An Stelle davon kann Grenzenlosigkeit treten. Wissen verliert jegliche Spezifika, da es sämtliche nur erdenklichen Informationen, Daten, Zeichen, Emotionen, Handlungsvollzüge, Kommunikationen subsumiert. Die daraus resultierende Orientierungslosigkeit lässt nicht erkennen, welche Bedeutung Wissen in einem Unternehmen zugesprochen werden sollte.

Es kann kein absolut zu erreichendes Wissen in Unternehmen geben, da die Wissensbasis eines Unternehmens selbst „inherently indeterminate and continually emerging" (Tsoukas, 1996, S. 22) ist. Unternehmen sollten lediglich ihre Aufmerksamkeit darauf lenken, kreative Köpfe und flexible Organisationsstrukturen zu forcieren und zu unterstützen, damit "knowing" als kulturelles Phänomen sich in den Handlungen bestmöglich entfalten kann (vgl. Blackler, 1993). 


\subsection{Management - der Navigator eines Unternehmens?}

Da sich Organisationstheorien als sehr inhomogen darstellen, wird auch dem Management eine in den einzelnen Theorien sehr divergierende Rolle zugesprochen. Lehnt man sich an das Framework von Burrell/Morgan (1979) an, so finden sich Organisationstheorien, die einerseits durch deterministische Ansätze sowie andererseits durch voluntaristische Ansätze geprägt werden. Erstere gehen davon aus, dass das Management prinzipiell keine Entscheidungsfreiheit und Wahlmöglichkeit hat, während letztere dem Management einen stärkeren Einfluss auf die grundsätzliche strategische Ausrichtung des Unternehmens zusprechen. Natürlich finden sich auch innerhalb dieser Unterteilung gemäßigte und radikalere Ansätze (vgl. Steyrer, 1999), was eine dezidierte Zuteilung von Organisationstheorien in eine der beiden Gruppierungen lediglich bedingt zulässt.

Unternehmen können ihr Handeln an externe Herausforderungen und Ansprüche anpassen und auf die einwirkenden Umweltveränderungen reagieren. Der Blickwinkel ist somit nach außen gerichtet und es wird untersucht, in welchen Situationen sich welche organisationalen Strukturen als ein optimaler "fit" darstellen. Denn Organisationen sind in objektiv zugänglichen Umwelten eingebettet und diese verwalten die knappen, aber für Unternehmen essentiellen Ressourcen. Somit müssen Unternehmen in ihren organisationalen Strukturen und Praktiken mit der Umwelt in Übereinstimmung sein, da sie ihre Produkte, sei es materieller oder immaterieller Natur, wiederum an diese zurück- und abgeben. Man unterstreicht in dieser Logik die Abhängigkeit eines Unternehmens von den Umweltgegebenheiten, die direkt und ausschließlich den unternehmerischen Erfolg bedingen (Meyer, 1996). Organisationstheorien, die dieser Klassifikation zugeordnet werden können, sind beispielsweise der Kontingenztheoretische Ansatz, Evolutionsorientierte Ansätze, der Population-Ecology-Ansatz bzw. entscheidungstheoretische Ansätze (Neumann, 2000). Auf eine Darstellung dieser Ansätze soll in der vorliegenden Arbeit verzichtet werden, da sie beispielsweise bei Kasper/Heimerl-Wagner (1996) oder Kieser (1999) nachgelesen werden können. 
Diese äußere Unternehmensperspektive lässt eine Binnenperspektive gänzlich außer Acht. Jedoch durch eine theoretische Ergänzung wie beispielsweise durch den "ressourcenbasierten Ansatz" (vgl. Peteraf, 1993; Penrose, 1959) wird der Fokus auf die Betrachtung der Innenwelt gelegt, indem Wissen als zentrale Ressource, als Kernkompetenz, als Quelle für Innovationen, als zentrales Element der Wertschöpfung und Bezugspunkt für das Organisieren von Lernprozessen anerkannt wird (vgl. Penrose, 1959; Hamel/ Prahalad, 1994, vonKrogh/ Ichijo/Nonaka, 2000; Neuman, 2000).

So wird der Schwerpunkt, der im Zuge von "marketbased theories" bzw. der Industrial-Organization-Forschung auf die Gefahren und Möglichkeiten, denen sich Unternehmen durch die Umwelt gegenübersehen, gesetzt worden ist, auf die Stärken und Schwächen von Unternehmen gelenkt. Es werden jene Faktoren hervorgehoben, auf die das strategische Management ausgerichtet sein soll, nämlich das Schaffen und Erhalten von strategischen Erfolgspotentialen, um mit Hilfe vorhandener Potentiale und Ressourcen Wettbewerbsvorteile zu schaffen und zu halten (Gälweiler, 1990). Dem Management wird mittlerweile eine aktive Rolle zugesprochen, das den Verlauf des Unternehmens beeinflussen kann, und der Erfolg des Unternehmens hängt nicht mehr ausschließlich von den Umweltgegebenheiten ab. Das Management erreicht eine Orientierung des gesamten Unternehmens auf die festgelegten Ziele nur durch eine Ausrichtung der Handlungen der beteiligten Akteure, wobei es sich bestimmter Instrumente bedienen kann und soll. So wird dem Management die Aufgabe zugesprochen, neben formalen Steuerungs- und Kontrollsystemen auch unternehmenskulturelle Symbole, beispielsweise organisationale Geschichten, Legenden, Anekdoten, zur zielorientierten Verhaltenssteuerung der Mitarbeiter einzusetzen (Kasper/ HeimerlWagner, 1996).

Klassische Methoden der Organisationsforschung basierten v.a. auf einer hierarchischen Kommandostruktur, der Standardisierung von Arbeitsabläufen und der Spezialisierung nach Funktionen als wesentliche Elemente einer effizienten Organisation. Anfang der neunziger Jahre konzentrierten sie sich auf die Verbesse- 
rung der operativen Arbeitsabläufe, indem Informations-, Planungs-, Entscheidungs- und Kontrollprozesse einer prozesstechnologischen Veränderung unterlagen. Organisationen wurden nicht mehr funktional gegliedert, sondern ablauforientierte Organisationsstrukturen ersetzten aufbauorientierte.

Durch die umbruchartigen Errungenschaften in den Informations- und Kommunikationstechnologien der letzten Jahre kam es vermehrt auch wieder in der Organisationsforschung zu Überlegungen, wie organisationale Strukturen geformt werden sollen, um den Herausforderungen und Spannungen, die durch das so genannte "Informationszeitalter" (Lash, 1994) hervorgerufen werden, gerecht zu werden und um gleichzeitig auch interne Ressourcen mit dem Schwerpunkt auf dem im Unternehmen vorhandenen Wissen optimal zu nutzen.

Ein optimaler interner sowie externer Fit wird im Rahmen neuer Organisationskonzepte angestrebt. Diese setzen bei ablauforientierten Konzepten an. Eine im gesamten Unternehmen implementierte technologische Basisstruktur gewährleistet, dass repetitive Tätigkeiten durch eine Bestgestaltung des Arbeitsplatzes effizient ausgeführt werden können, indem Arbeitsverrichtungen und der Materialsowie Informationsfluss am einzelnen Arbeitsplatz durch die Verfügbarkeit von PCs und Intranets etc. unterstützt werden. Diese Automatisierungstechnik muss jedoch vermehrt auch die sog. „Wissensarbeit" mit ihren Eigenschaften wie hoher Qualifikation der Mitarbeiter, Problemlösungen im Team, abteilungsübergreifender Projektarbeit, starker Kundenorientierung sowie einer Koordination unter Gleichgestellten anstatt von Top-down-Vorgaben berücksichtigen, was die Suche nach neuen Organisationsformen legitimiert (Pinchot/Pinchot, 1996).

Innerhalb von voluntaristisch geprägten Ansätzen wird die Annahme vertreten, dass Unternehmen ihren Kontext als nicht gegeben hinnehmen, sondern ihr Umfeld interpretieren, um auf unveränderbare Umfeldveränderungen reagieren zu können und veränderbare Elemente bewusst zu gestalten. Es wird die Objektivität einer positivistischen Wissenschaftsauffassung aufgegeben und akzeptiert, dass es nur eine vom Beobachter konstruierte Wirklichkeit gibt. Die organisatio- 
nale Realität wird über eine diskursive Verständigung intersubjektiv geteilter Bilder und Vorstellungen erzeugt. Damit existiert die Realität, radikal ausgedrückt, lediglich in den Kognitionen der Organisationsmitglieder. Drei generelle Richtungen konstruktivistischer bzw. interpretativer Ansätze werden in den Organisationstheorien unterschieden. Eine sozial-konstruktivistische, die stark von Berger/Luckmann beeinflusst ist, eine kognitive und eine systemtheoretische. Erstere basiert auf einem grundlegenden Misstrauen gegenüber Fakten und gegenüber Organisationstheorien, die sich an Fakten orientieren. Ansätze, die dieser Logik zuzurechnen sind, sehen ihre Aufgabe letztlich darin, die Relativität, Bedingtheit von Organisationsstrukturen sichtbar zu machen - sie zu „dekonstruieren“ (Kieser, 1999).

Ansätze eines kognitiven Konstruktivismus hingegen versuchen die positivistische und die interpretative Richtung miteinander zu verbinden, indem sie das Verhalten von Individuen in einem hohen Maße von subjektiven Theorien, die subjektive Annahmen über Kausalitäten und einfachen Regeln, die im Gedächtnis gespeichert sind, umfassen, gesteuert sehen. Solche Kognitionen können auf positivistische Weise durch bspw. Interviews rekonstruiert werden.

Systemtheoretische Ansätze konzentrieren sich auf die Frage, wie Menschen unter Berücksichtigung ihrer neuro-physiologischen Ausstattung Erkenntnisse gewinnen (Kieser, 1999). Organisationen werden als symbolisch-ideelle Phänomene angesehen, die in den Kognitionen der Akteure einer Organisation als „Realität" konstruiert werden. Die Bedeutungsinhalte und konkreten Erscheinungsformen von Organisationen lassen sich nicht ohne weiteres aus Organisationszielen oder Organisationsstrukturen ablesen. Es gilt die weitaus tiefer liegenden Mechanismen, die auf das Handeln und Erleben in einer Organisation einwirken, zu ergründen. So sieht man sich dem zugrunde liegenden Sinnsystem gegenüber, das als ein Beziehungsgeflecht von symbolischen Kommunikationsund Interaktionsprozessen verstanden wird und das es zu ergründen gilt (Kasper/Heimerl-Wagner, 1996). In einer derartigen Betrachtungsweise wird der Fokus auf den Menschen als zentralen Bestandteil von Organisationen überwun- 
den. In den Mittelpunkt rücken Kommunikationen als Bausteine sozialer Systeme (Kasper/ Mayrhofer/Meyer, 1999).

Eine derartige gedankliche Ausrichtung entfaltet Auswirkungen auf die Steuerung von Unternehmen. Wenn Kommunikationen ausschließlich auf Kommunikationen reagieren, scheint eine Lenkung durch einen außenstehenden "Faktor" nicht mehr möglich und eine Steuerung gänzlich im Inneren des sozialen Systems stattzufinden. Diese Selbststeuerung wird jedoch sehr wohl durch Handlungen von Managern beeinflusst werden, da die prinzipielle Möglichkeit der Beeinflussung durch Manager auch im Rahmen dieser Logik außer Frage steht (Kasper/ Mayrhofer/Meyer, 1999). Trotz der energetischen Offenheit bezieht sich das System in jeder seiner Operationen rekursiv auf sich selbst (Meyer, 1996) und das Eingreifen in das soziale System ist nur über strukturelle Veränderungen des Systems möglich. Manager müssen sich dabei Kopplungsmechanismen, die zwischen der Umwelt und dem System liegen, bedienen, um die Veränderungsprozesse überhaupt hervorrufen zu können. Beispielsweise können sie dafür Personen nutzen (Kasper/Mayrhofer/Meyer, 1999). 


\subsection{Wissen managen - eine symbiotische Beziehung oder ein Wi- derspruch in sich?}

Der in den letzten Jahren vermehrte Zuwachs an weiterentwickelten, dynamischen und innovativen Managementlösungen und -techniken ließ die Hoffnung steigen, neue und praktikablere Wege gefunden zu haben, mit denen Unternehmen noch mehr Leistung hervorbringen, einen noch größeren komparativen Vorteil gegenüber den Mitbewerbern erlangen und intern auf eine noch ausgeklügeltere Weise strategische Ressourcen aufbauen können (im Sinne von Peteraf, beispielsweise 1993). Auch Wissensmanagement ist an dieser Stelle als Lösungsansatz zu nennen. Dieser Forschungsbereich fügt die den Teilbereichen Wissen und Management zugrunde liegenden Annahmen in einer Disziplin zusammen und dringt vor allem über die Unternehmensberatungsbranche in die berufliche Welt ein, erhebt auf die eine oder andere Art den oben definierten Anspruch und legt dadurch das Fundament für Umstrukturierungen, für eine Neuorientierung auf organisationaler Ebene und für einen organisatorischen Wandel.

Die Organisation, als Managementfunktion betrachtet, schließt sämtliche Entscheidungs- und Durchführungsprozesse ein, die in einem mehr oder weniger formalisierten Ordnungsmuster (Struktur) als Mittel (Instrument) zur möglichst dauerhaften Lösung von Systemproblemen (Institution) resultieren sollen (Heinl, 1996 in Neumann, 2000). Diese Prozesse werden durch WissensmanagementKonzepte zu unterstützen und zu optimieren versucht.

Zu saturiert scheinen jedoch die Wissenschaft und die Wirtschaft bereits von diesen „neuen" Managementmethoden zu sein, die sämtliche Probleme zu lösen versprechen, indem sie das betriebliche Umfeld in Kausalzusammenhänge stecken, die lediglich des richtigen Inputs bedürfen, um zum gewünschten Output zu gelangen. Bei mangelndem Agreement, was nun eigentlich unter ${ }_{n}$ Wissensmanagement" verstanden werden kann und welche Zielvorstellungen damit einhergehen, kann scheinbar ein „passender" Input nicht definiert werden, um einen für Unternehmen „passenden" Output zu schaffen. 
"Probably it would be generally agreed that the primary economic function of an industrial firm is to make use of productive resources for the purpose of supplying goods and services to the economy in accordance with plans developed and put into effect within the firm" (Penrose, 1997, S. 30). Jedoch "[s]trictly speaking, it is never resources themselves that are the 'inputs' in the production process, but only the services that the resources can render" (Penrose, 1997, S. 31).

Welche "services" von Ressourcen in Anspruch genommen werden, hängt von deren Nutzung und Verwendung ab. Einzelnen Unternehmen steht dadurch die Möglichkeit offen, sich von anderen zu unterscheiden, wodurch sie ebenfalls einen komparativen Vorteil gegenüber der Konkurrenz ausbauen bzw. initiieren können (vgl. Penrose, 1997). Das Management übt hier einen großen Einfluss aus, da "existing managerial personnel provide services that cannot be provided by personnel newly hired from outside the firm [...], but also because the experience they gain from working within the firm and with each other enables them to provide senvices that are uniquely valuable for the operations of the particular group with which they are associated" (Penrose, 1997, S. 33). Auch wenn das Management selten unmittelbar selber in den Produktionsprozess bzw. den Prozess des Gebens von Dienstleistungen involviert ist, so ist es doch die Einheit eines Unternehmens, die die Entscheidungsbefugnis über das Einsetzen von Ressourcen und die Art und Weise, wie dies erfolgen soll, zugesprochen bekommt.

Im Zuge von Wissensmanagement sieht sich ein Management nun des Managens von Wissen gegenüber, wobei sich dies unterschiedlich gestalten kann. Der Grund hierfür ist innerhalb der Organisationen selbst zu finden, die oftmals einen untereinander stark divergierenden Mix an Wissenstypen aufweisen. Je nachdem, auf welche Wissenskategorie ein Unternehmen seine Hauptaufmerksamkeit richtet, werden die organisationalen Strukturen ausgerichtet (Lam, 2000) und es gestaltet sich dadurch ein Wissensmanagement. Nimmt man die vier Wissenstypen („embrained knowledge", „embodied knowledge", „encoded knowledge" sowie "embedded knowledge"), wie sie in Kapitel "Wissen - DIE Ressource oder 
Resultat von Interaktion?" dargestellt wurden, so erhält man auch vier mögliche Ansätze, wie Wissensmanagement in einem Unternehmen betrieben werden könnte (vgl. Lam, 2000).

So neigen Organisationen, die ihre Wettbewerbsvorteile vorrangig durch das einzigartige Wissen in Form des "embrained knowledge" einzelner Experten des Unternehmens lukreieren, dazu, formale Strukturen mit Kontroll- und Koordinationsmechanismen sowie standardisierten Arbeitsabläufen zu haben. Auch wenn den einzelnen Experten grundsätzlich eine Entscheidungsfreiheit und gewisse Autonomie erhalten bleibt, so zeichnet sich generell ein derartiges Unternehmen durch seine bürokratischen Strukturen aus, was auch der Name "professional bureaucracy" zum Ausdruck bringt, den man dieser Art von Unternehmen zuweist. Die Hauptträger von Wissensmanagement sind in diesem Fall die Experten des Unternehmens. Durch die funktionale und hierarchische Strukturierung des Unternehmens kommt es nur eingeschränkt zu einer Wissensteilung über Abteilungsgrenzen hinweg; Expertenwissen wird lediglich in speziellen Bereichen eingesetzt und kommt selten dem gesamten Unternehmen zugute. Dies bedingt auch die eingeschränkte Auseinandersetzung mit implizitem Wissen.

Unternehmen, die ebenfalls stark von explizitem Wissen abhängig zeichnen, jedoch von explizitem Wissen auf organisationaler Ebene, basieren auf den Schlüsselprinzipien von Spezialisierung, Standardisierung und Kontrolle und werden "machine bureaucracy" genannt. Das formale, explizite Wissen konstituiert die Basis von internen Richtlinien und einem Rahmengerüst, innerhalb dessen Arbeiten erledigt werden. Das Wissensmanagement kommt vor allem dem Management selbst zu, das auf höherer Hierarchieebene, organisationales Wissen in Richtlinien und Standardverfahren verankert, wodurch eine Unsicherheit innerhalb des Unternehmens reduziert werden kann und Routineaufgaben bestmöglich gemeistert werden können. Auch hier wird dem impliziten Wissen eine untergeordnete Rolle zugesprochen. 
Dem konträr gegenüber steht eine Organisationsform, die vor allem das implizite, individuelle Wissen, das Know-how und die praktischen Problemlösungsfertigkeiten von Experten, zu managen versucht. Mittels einer "operating adhocracy", das sich durch eine starke Interaktion zwischen den Mitarbeitern auszeichnet, wird das Unternehmen vor allem im Sinne von "trial-and-error" sowie einer "Experimentierfreudigkeit" geführt. Wiederum erhalten die Experten eines Unternehmens die größte Aufmerksamkeit im Rahmen des stattindenden Wissensmanagements.

Eine "J-Form Organisation" fokussiert sich auf das "embedded knowledge" und hier kommt weder dem einzelnen Individuum noch dem Management selbst die tragende Rolle im Rahmen des Wissensmanagements $\mathrm{zu}$, sondern dem Projektteam, das in dieser Organisationsform hervorgehoben wird. Durch Teamarbeit und abteilungsübergreifende Zusammenarbeit wird gemeinsame Problemlösung angestrebt, was gleichzeitig den Austausch von explizitem sowie von implizitem Wissen fördert.

Alles in allem zeigt sich in einem funktionalistisch geprägten Paradigma, dass ein Unternehmen ein Wissensmanagement hat, unabhängig davon, wem die Rolle des Managens von Wissen zugesprochen wird bzw. wer die Hauptträger von Wissen sind. Der Praktikabilität der entwickelten Ansätze wird Beachtung geschenkt und es werden ökonomisch verwertbare Lösungen von Wissensmanagement ausgearbeitet. Dabei kommt vor allem dem Teilbereich Management eine bedeutende Rolle zu, da durch dieses das Personal und somit der Verlauf des Unternehmens bestimmt bzw. gesteuert werden können.

Eine interpretative Logik, die den Akteuren ihre soziale Realität konstruieren lässt, stellt das Handeln und die subjektiven Sinngehalte der Akteure in den Mittelpunkt der Betrachtung. Das Verständnis wird aufgegeben, dass eine objektive Wirklichkeit dem Menschen zugänglich sei. Wissensmanagement in einem solchen Paradigma erschließt sich in Annäherungen an die sozialen Strukturen und Prozesse, mit denen Wissen geteilt und generiert wird. Beispielhaft sollen hier die 
"Communities of Practice" genannt werden (vgl. beispielsweise die Werke von Wenger, 1998; Brown/Duguid, 1998, 2000; Wenger/Snyder, 2000; Lave/Wenger, 1991; Gherardi/Nicolini/Odella, 1998). Diese werden durch eine Gruppe von Leuten gebildet, die zusammen im Rahmen gemeinsamer Praxis Wissen generieren und teilen. Alleine durch den Term "Communities of Practice" soll die Bedeutung der Beziehung zwischen einer Handlung und Wissensgenerierung innerhalb solcher Gruppen betont werden. Diese Communities stellen das soziale Kapital eines Unternehmens dar. Dabei wird soziales Kapital als "relationships that make the organisation work" (Prusak/Cohen, 2001) definiert.

Hierbei gilt "... [Communities of practice] should not be interpreted as assuming that knowing is all that communities of practice are about, especially if by "knowing" one refers to some instrumental kind of expertise. Communities of practice should not be reduced to purely instrumental purposes. They are about knowing, but also about being together, living meaningfully, developing a satisfying identity, and altogether being human" (Wenger, 1998, S. 134).

Dieser Form von Communities wurde in der jüngeren Vergangenheit große Beachtung geschenkt und Bedeutung zugesprochen, als die Tatsache mehr und mehr bewusst wurde, dass Mitarbeiter dazu tendieren, sich mit fachlich nahe gestellten Kollegen zu vernetzen und Problemstellungen erst nach Einbeziehung des Rats anderer zu lösen, auch wenn sie räumlich und/oder organisatorisch verteilt sind und nicht in gemeinsamen Arbeitsprozessen tätig sind. Diese gemeinsam geformte und konstruierte Arbeitswelt bildet den Rahmen, in dem Lernprozesse stattfinden. Ferner erweitern sich durch eine derartige soziale Interaktion der Betrachtungshorizont sowie Handlungsspielraum der Akteure, da die Teilnehmer eine Community of Practice sich abteilungsübergreifend organisieren und dabei ihre formalen Arbeitsbereiche verlassen werden (vgl. Schön, 2001; Schütt, 2000; Wenger/Snyder, 2000; Brown/Duguid, 1991).

Communities of Practice erweisen sich jedoch als schwer greifbar. Betrachtet man die Aktivitätssysteme einzelner Akteure, so finden sich unterschiedlichste 
Arten von derartigen Communities, sei es im beruflichen oder privaten Umfeld, denn "[i]n fact, communities of practice are everywhere" (Wenger, 1998, S. 6). Diese stellen einen integralen Teil des täglichen Lebens dar, fortwährend in verschiedener Gestalt auftretend. Auf Grund der Informalität und Abstraktheit der Communities sind sie nur selten explizit Gegenstand von Diskussionsprozessen der Teilnehmer solcher Gemeinschaften. Implizit bei den einzelnen Akteuren besteht kein Zweifel darüber, wer als Mitglied zu einer Community zählt, ohne dass diese sich im Aktivitätssystem selbst explizit als solche ausweisen müssten.

Es findet sich ein unausgesprochenes Verständnis von beispielsweise Rollenverteilung, zugrunde gelegten Annahmen oder stillen Konventionen (vgl. Wenger/ Snyder, 2001; Henschel, 2001). Das Wechselspiel zwischen stillen und ausgesprochenen Übereinkünften innerhalb einer Community charakterisiert eine solche. "Communities of practice are the prime context in which we can work out common sense through mutual engagement. Therefore, the concept of practice highlights the social and negotiated character of both the explicit and the tacit in our lives" (Wenger, 1998, S. 47).

So kommt es, dass sich auf informeller und selbst-organisatorischer Basis Individuen aus einem gemeinsamen Interesse heraus zusammenschließen und in einem abgegrenzten Themengebiet ihr Wissen austauschen sowie gemeinsam entwickeln und Problemlösungen erarbeiten (vgl. Schön, 2001). Doch nicht nur der Austausch von Ideen, Erkenntnissen und Erfahrungen steht im Mittelpunkt einer Community of Practice, sondern sehr wohl auch die gegenseitige Hilfe und Unterstützung, wodurch erst ein gemeinsames Lernen ermöglicht wird (vgl. Wenger/Snyder, 2000). Hierbei können sich die Beiträge und die Mitwirkung der einzelnen Akteure sehr stark unterscheiden, da durch die Selbstorganisation der Community keine Verpflichtung in irgendeiner Art besteht. Auf dieser freiwilligen Basis erfolgt auch eine Zielsetzung, wobei der Zweck der Community oftmals stillschweigend als ohnedies gemeinhin bekannt angenommen wird und unausgesprochen die Aktivitäten der Akteure umspannt. Werden intrinsische Motivationsfaktoren erfüllt, schöpfen Mitarbeiter daraus Motivation, wodurch sie das ein- 
gebrachte Engagement stetig steigern werden. Solange sie an der Aufrechterhaltung der Community interessiert sind, werden sie ihr Wissen und ihre Arbeitskraft dort einsetzen, ohne je formal Verantwortlichkeiten übernehmen zu müssen (vgl. Henschel, 2001). Diese interne Struktur und Organisation einer Community of Practice grenzt sich von anderen Formen von Arbeitsgruppen (wie beispielsweise Teams, Projektgruppen, Netzwerke) klar ab (eine detaillierte Ausführung von Unterschieden findet sich beispielsweise bei Henschel, 2001).

Durch eine unternehmensweite Vernetzung von Mitarbeitern ungeachtet ihrer Hierarchiestufe oder ihrer räumlichen Position werden Informationen ausgetauscht, die auf rein formalem Wege meist nicht zugänglich wären. So unterstützt eine flache Hierarchie innerhalb der Communities die Reflexion der Tätigkeiten und der hohe Praxisbezug lässt optimale Lösungsansätze für fachliche Problemstellungen entstehen. Nicht verwunderlich scheint die Tatsache, dass derartige Communities Innovationsquellen darstellen, da die Grundcharakteristika dieser Art der Zusammenarbeit genau einen Rahmen bieten, in dem die Verbreitung von neuen Ideen eine natürliche Erweiterung der vorhandenen Funktion darstellt (vgl. Senge, 2000; Eppler/Sukowski, 2001; Wenger/Snyder, 2000).

Die hier skizzierte Form von Communities of Practice zeigt vor allem für die sich darin weiterentwickelnden Akteure positive Entfaltungs- und Gestaltungsmöglichkeiten, was sich jedoch einem Management nur sehr schwer offenbart. Oftmals stehen die Austauschprozesse innerhalb einer Community sogar im Widerspruch zu den Managementvorgaben, wodurch die Aktivitäten solcher Communities auch unter dem Namen „Bootlegging-Prozesse" (Zucker/Schmitz, 2000) bekannt sind. Die Notwendigkeit seitens des Managements ist daher gestiegen, Versuche zu unternehmen, Wissen von Mitarbeitern bewusst und systematisch auch über Communities of Practice zu erschließen. So sind beispielsweise in internationalen Konzernen wie DaimlerChrysler, Ford, IBM, Siemens, Shell, Volkswagen oder Xerox die systematische Etablierung und Förderung von Communities of Practice explizit in die Unternehmensstrategien aufgenommen worden (Schön, 2001, S. 60). 
Ein solches Vorgehen läuft jedoch den charakteristischen Eigenheiten der Communities zuwider, da sich diese ja beispielsweise durch das Nichtvorhandensein von formaler Führung und Steuerungsmechanismen auszeichnen. Versucht man die intrinsische Motivation und das Eigenengagement in Beurteilungs- und $\mathrm{Be}-$ förderungssysteme zu integrieren (wie es beispielsweise die American Management Systems oder die Weltbank versucht haben, Wenger/Snyder, 2000, S. 145), so untergräbt man damit jegliche Legitimation solcher Gemeinschaften, da sich diese grundlegenden Aspekte wie Selbstorganisation, Eigenengagement oder intrinsische Motivation nicht mehr bilden können, die jedoch im Rahmen einer natürlichen Entstehung von Communities of Practice als Fundament dienen (vgl. Brown/Duguid, 1991).

Betrachtet man Communities of Practice jedoch von einem funktionalistischen Gesichtspunkt aus, so wird von der Annahme ausgegangen, dass sich das Wissen, das in solchen Gemeinschaften generiert sowie transferiert wird, als für das jeweilige Unternehmen erschließbar darstellt. Somit wird der Analyse der Communities of Practice eine besondere Bedeutung beigemessen.

Basierend auf dieser Grundannahme wird in Communities of Practice eine spezielle Form der Konversation, nämlich die des Story-Telling, ausgemacht, die verwendet wird, um die in einem Unternehmen informell stattfindenden Interaktionsprozesse zu erschließen. Unter "Story-Telling" wird gemeinhin eine Methode verstanden, mit der Individuen Ereignisse und Erfahrungen so präsentieren, wie sie in den einzelnen Gedankenkonstrukten erlebt worden sind ( $\mathrm{vgl}$. Czarniawska, 1998): „[stories] infuse events with meaning [...] through the magic of plot" (Gabriel, 2000). Geschichten nehmen hierbei eine Schlüsselfunktion bei der Informationsteilung in Unternehmen ein und prägen die Identität einer Gemeinschaft.

Dem Gewöhnlichen, das als "normal" und "erwartet" angesehen wird, ist eine gewisse Legitimität und Autorität inhärent. Genau diese Rationalität findet sich jedoch bei Geschehnissen, die Gegenstand einer story " werden, nicht, wodurch 
durch den Prozess des "Story Telling" ein "sensemaking" (Weick, 1995) initiiert wird, das ein neues Ereignis in die allgemein gültige Wirklichkeitskonstruktion integriert und dadurch verständlich macht. Denn Geschichten dienen dem Zweck, eine Verbindung zwischen dem Außergewöhnlichen und dem Gewöhnlichen herzustellen (vgl. Czarniawska, 1998).

Dabei unterliegen Geschichten auch einem Veränderungsprozess, der sich für den Urheber als meist nicht beeinflussbar gestaltet (vgl. Gabriel, 2000), und es kommt fortwährend zu einer kritischen Durchleuchtung des als rational Akzeptierten. Das Ungewusste wird immerfort unbewusst strukturiert sowie die Art und Weise, wie Individuen ihre sozialen Realitäten konstruieren, hinterfragt. Dadurch erhalten Geschichten eine nicht antastbare Macht, da sie auf eine Ebene transformiert werden, die derartig in Unternehmensnetzwerken gefestigt ist, dass das Erlebte in Verhandlungsprozessen verankert und weitreichend erinnerbar bleibt (vgl. Bruner, 1990).

Kommunikation in einer solchen Weise geht über das Teilen von Informationen und Daten weit hinaus und vor allem fachliches Wissen und Problemlösungsvorgänge werden in Form von "stories" geteilt (vgl. Gabriel, 2000; Spender, 1996). Indem praktisches Wissen durch "Story-Telling" weitergeleitet wird, können sämtliche Mitglieder einer speziellen Community of Practice auf dieses Wissen zugreifen und damit arbeiten sowie dieses erneuern, ergänzen oder einfach durch neues Wissen ersetzen, was zu neuen Geschichten führt. Das Wissen, das hier Gegenstand des Austausches zwischen den Teilnehmern einer Gemeinschaft ist, ist jenes, das in expliziter Form transferiert werden kann.

Die mit einer Community of Practice einhergehende Arbeitsatmosphäre fußt vor allem auf einem Umfeld, in dem Vertrauen einen wichtigen Stellenwert einnimmt und erst das wechselseitige Verstehen ermöglicht, dass Lernprozesse und Prozesse der Wissensgenerierung und -teilung initiiert werden können (vgl. Cohen/Prusak, 2001; Sydow, 1992). "Story-Telling“ kann sich genau in einem solchen Umfeld entfalten, wobei es nicht verständliche Vorgänge in weithin akzep- 
table und logische transformiert. Auch werden Geschichten dazu verwendet, die Charakteristiken einer Gemeinschaft festzuhalten, indem Gefühle wie Interesse, Freude, Begeisterung, aber auch Furcht oder Wut eingearbeitet werden. Dadurch findet die emotionale Sensibilität Berücksichtigung und auch Legitimität. In einem streng auf funktionalistischen Annahmen ausgerichteten Unternehmen scheint diese Art der Kommunikation völlig den Steuerungs- und Kontrollmechanismen des Managements zuwider zu laufen (vgl. Alvesson/Karreman, 2001). Wo vormals gemeinhin akzeptiert worden ist, dass der Erfolg eines Unternehmens von rationalen und meist mechanischen Problemlösungen abhängig ist und auf klar dokumentierte Verfahren und Richtlinien zurückgegriffen worden ist, schien für menschliche Emotionen nur wenig Raum übrig zu bleiben. Genau dies wurde beispielsweise durch die Formierung von Communities of Practice überwunden. Denn die Ausrichtung auf eine in dieser Gemeinschaft übereinstimmende Zielsetzung wird durch den informellen Charakter und den damit einhergehenden intrinsischen Motivationsfaktoren sowie durch das Einbinden von "stories" mit einem weitaus emotionalen Arbeiten verbunden. Eine Identifizierung mit der Arbeit erfolgt auf einer gefühlsbetonten Ebene (vgl. Denning, 2001).

Im Zuge einer gemeinsamen Realitätskonstruktion kommt es innerhalb einer Community of Practice auch zu einer Abgrenzung von anderen Gemeinschaften und es erfolgt eine klare Einbettung in das Unternehmensumfeld. Dadurch wird der Prozess, Daten, Informationen und Wissen sowie Erfahrungen und Geschehnisse in eine Beziehung zu den formalen Aktivitäten eines Unternehmens zu bringen, erleichtert.

Versucht man sich von der Ebene des Managements aus (d.h. von einer Makroperspektive), das Wissen, das innerhalb der Communities of Practice geteilt und generiert wird, zugänglich zu machen, so bietet die Methode des "knowledge mapping" eine Hilfestellung, die "knowledge environment" (Venters, 2002) besser zu verstehen, in der die Wissensprozesse stattfinden. 
Die Art des Vorgehens im Zuge des "knowledge mapping" wurde im Feld des Knowledge Engineering und im Rahmen des Forschungsbereichs der Künstlichen Intelligenz entwickelt und zeigt nochmals, dass von der paradigmatischen Fundierung aus diese Ausführungen sehr stark auf den Annahmen einer funktionalistisch orientierten Logik gründen.

Im Rahmen des "knowledge mapping" wird versucht, die Wissenspotentiale eines Unternehmens entlang einer Wertkette aufzuschlüsseln und durch diese Strukturierung klar zu visualisieren, welcher Teil eines Unternehmens einer besonderen Aufmerksamkeit bedarf (vgl. Schüppel/Müller-Stewens/Gomez, 1998). Man ist sich des Vorhandenseins von Communities of Practice bewusst, möchte die dort stattfindenden Wissensprozesse jedoch dem formalen System der Organisation zugänglich und nutzbar machen. Dabei werden Topographien des Wissens erstellt, auf denen all das verzeichnet ist, was sonst nur in Köpfen oder kollektiven Systemen vorkommt. Zunächst müssen dafür wissensintensive Prozesse erfasst werden, um daraus relevante Wissensbestände ableiten zu können. Dieses in sog. "Wissenskarten" abgelegte Wissen wird anschließend mit den (wissensintensiven) Arbeitsprozessen verknüpft. Dadurch wird jedem Einzelnen eine Hilfestellung angeboten, mit Situationen und Handlungsalternativen so umzugehen, dass Ziele erreicht werden können. Wissenskarten sollen sich daher nicht auf Repräsentationen und Abbilder von Umwelten und Zusammenhängen reduzieren, sondern den Handlungsrahmen der Organisationsmitglieder abstecken sowie die soziale und mikropolitische Ordnung in einem Unternehmen zeigen ( $\mathrm{vgl}$. Roehl, 2002; Neumann, 2000).

Im Zuge des "knowledge mapping" steht jedoch weniger im Vordergrund, die organisationalen Wissensstrukturen exakt abzubilden als vielmehr eine allgemein akzeptierte Argumentationsbasis in der Organisation zu schaffen. Sie tragen zu einer Transparenz bei und machen Erkenntnisse der Vergangenheit für jedermann zugänglich. Doch genau dieser Punkt ist es, der als kritisch zu betrachten ist. Denn Wissenskarten können nur den expliziten Teil von Wissen einer organisationalen Wissensbasis zuführen und nach plausiblen Logiken aufschlüsseln. 
Somit wird Wissen, das durch den Prozess des "knowing" generiert wird bzw. das als implizit einzustufen ist, nur marginal behandelt bzw. findet es überhaupt keine Berücksichtigung. Im Zuge einer fortwährenden Aktualisierung der Wissenskarten kann die dieser Methode grundsätzlich inhärente Statik jedoch teilweise überwunden werden (vgl. Roehl, 2002).

Mit Personalstrategien wird versucht, diejenigen Mitarbeiter einzustellen, von denen man sich erwartet, dass sie sich schnell sozialisieren können und mit denen Personennetze gespannt werden, die Communities of Practice entstehen lassen. Durch eine derartige Förderung dieser informellen Gemeinschaften versucht das Management, sich genau das zunutze zu machen, was unter "normalen" Umständen als nicht akzeptabel eingestuft wird: nämlich Interaktionsprozesse, die sich der formalen Steuerung entziehen und nachweisbar nicht direkt auf die Unternehmensziele ausgerichtet sind. Diese Communities of Practice werden sodann als eine neue Ebene der Organisation betrachtet:

"Communities of Practice are the new frontier. They may seem unfamiliar now, but in five to ten years they may be as common to discussions about organization as business units and teams are today - if managers learn how to make them a central part of their companies' success" (Wenger/Snyder, 2000, S. 145).

Dennoch kann nicht überzeugend die Diskrepanz überwunden werden, dass Communities of Practice, wenn schon nicht informell und auf evolutionärer Basis, geplant und formell entstehen, ihre typischen Charakteristika (wie beispielsweise Selbststeuerung, intrinsische Motivation und dergleichen) verlieren. Sicherlich wird man ein Umfeld konstruieren können, die die darin entstehenden Gemeinschaften dann als genuin entstandene Communities erscheinen lassen, jedoch sollte man auf der Unternehmensebene die Erwartungen nicht allzu hoch schrauben, da zumindest laut den Annahmen eines interpretativen Paradigmas immer davon ausgegangen wird, dass es Wissensprozesse gibt, die sich einem Management als nicht zu erschließen zeigen. 
Entstehen diese Gemeinschaften in der Form, wie es ursprünglich verstanden wurde (vgl. Wenger/Snyder, 2000), so geht dies mit Annahmen eines interpretativen Paradigmas einher, in dem sich Wissensmanagement dann als etwas, was ein Unternehmen ist, erweist. Sämtliche Versuche, Communities of Practice durch das Management initiieren und gestalten zu lassen, schließen an wissenschaftstheoretische Annahmen eines funktionalistisch orientierten Paradigmas an. Andererseits kann durch die unternehmensweite Auseinandersetzung mit dieser Thematik eine Atmosphäre geschaffen werden, die Mitarbeiter dazu motiviert, ihre fachliche Expertise in Communities einzubringen. Dadurch erfährt die Arbeit des Einzelnen eine gewisse Wertschätzung durch das Management, wodurch sich dies sehr wohl positiv auf den Verlauf des Erfolgs eines Unternehmens auswirken kann (vgl. Henschl, 2001; Schön, 2001; Wenger, 1998). 


\subsection{Exkurs: Organisationales Lernen}

Organisationales Lernen wird in dieser Arbeit als Seitenstrang des Forschungsbereichs Wissensmanagement behandelt ( $\mathrm{vgl}$. Venters, 2001). Würde man sich rein mit diesem Feld auseinander setzen wollen, könnte man alleine damit eine ganze Dissertation füllen. Daher wurde entschieden, hier eine Eingrenzung vorzunehmen. Fragestellungen dieser Dissertation werden durch die Brille "Wissensmanagement" betrachtet, weshalb Forschungsresultate des Bereichs ${ }_{n}$ Organisationalen Lernens" lediglich tangiert werden.

Betrachtet man die sozialen und menschlichen Aspekte des Wissensmanagements näher, scheint man Antworten auf Fragestellungen ausschließlich im Bereich des organisationalen Lernens zu finden. Dieser Forschungszweig hat über die letzten Jahre auf dem Gebiet des Individuums, d.h., wenn die Person im Mittelpunkt der Betrachtung steht, eine Vorrangstellung erlangt, was vor allem mit der Publikationsflut in diesem Bereich gezeigt werden kann. Obwohl in den Ausführungen des organisationalen Lernens vor allem auf die Pionierwerke Ende der Fünfziger, Anfang der Sechziger und späten Siebziger rekurriert wird (vgl. Cangelosi/Dill, 1958; Simon, 1957; Cyert/March, 1963; March/Olsen, 1976; Argyris/ Schön, 1978; Duncan/Weiss, 1978), kam es erst in den späten Achtzigern und vor allem seit Anbeginn der neunziger Jahre zu zahlreichen Publikationen auf diesem Gebiet.

Im Bereich Wissensmanagement findet man selten den Versuch, dass personale Problemstellungen nicht mit organisationalen Lerntheorien und damit einhergehenden Annahmen zu ergründen. Es verwundert daher auch nicht, dass, sobald die Aufmerksamkeit im Bereich Wissensmanagement auf soziale Aspekte und die Einbindung des Individuums erfolgt, lerntheoretisch argumentiert wird und oftmals keine Unterschiede mehr zu dem Gebiet des organisationalen Lernens festzustellen sind. 
In der gängigen Literatur des organisationalen Lernens lässt sich keine einheitliche Definition dieses Begriffes ausmachen (vgl. Argyris/Schön, 1978). Dies erschwert es, sich einen Überblick über den Forschungsgegenstand dieses Bereiches zu verschaffen. Daher waren die diesem Forschungsgebiet zugehörigen Ansätze oftmals Gegenstand eines Versuchs der systematischen Darstellung, beispielsweise zu finden bei Shrivasta (1983); Dodgson (1993), Easterby-Smith (1997), Tsang (1997), Schreyögg/Eberl (1998), Easterby-Smith/Araujo, (1999), Prange (1999) oder Elkjaer (1999). Dabei haben die Autoren insbesondere versucht, die Beiträge aus den unterschiedlichsten Disziplinen zu klassifizieren und typologisieren.

Der Forschungsbereich des organisationalen Lernens stellt sich als eine Gestaltungslehre zur Anleitung zu organisationalen Lernprozessen dar, wobei die Unterscheidung zwischen behavioristischen und kognitivistischen Lerntheorien eine Erwähnung erfahren soll. Diese Zweiteilung hat zahlreiche Versuche geprägt, Lernkonzepte zu klassifizieren.

Behavioristische Lerntheorien konzentrieren sich auf die beobachtbaren Verhaltensmodifikationen von Organisationen. Dabei wird Individuen ein behavioristisches Verhaltensgrundmodell zugrunde gelegt und diese konzipierte Theorie auf Organisationen übertragen, was für Organisationen bedeutet, dass sie somit in einem engen Wechselverhältnis zur Umwelt stehen. Umweltveränderungen innerhalb der Organisationskontexte werden identifiziert, worauf adaptiv reagiert wird. Kognitive Elemente werden einzubeziehen versucht, jedoch das Grundkonzept wird von einem Stimulus-Response-Gesichtspunkt aus dominiert und das Individuum als Black Box betrachtet.

Organisationales Lernen wird als der Prozess verstanden, mit Hilfe dessen Informationen - sei es von innerhalb oder außerhalb des Unternehmens - aufgenommen und interpretiert werden können, um passend darauf zu reagieren ( $v g l$. Huber, 1991; vgl. Argyris/Schön, 1978). Ein langfristig komparativer Vorteil gegenüber Mitbewerbern kann nur dann erreicht werden, wenn Unternehmen ihre 
Stärken kanalisieren, um schnell bzw. „besser" als die Konkurrenz zu lernen (Collis, 1994; Grant, 1996). Organisationales Lernen stellt hierbei in den Pionieransätzen von Cyert/March (1963), Argyris/Schön (1978) und March/OIsen (1988) einen zyklischen systematischen Prozess dar. Das sich immer schneller verändernde Umfeld muss bewältigbar sein und Theorien des organisationalen Lernens sollen Unternehmen dabei unterstützen, intern Verbesserungspotentiale zu entdecken. Es gilt Fehlerabweichungen und Zielkonflikte in der strategischen Ausrichtung des Unternehmens zu berücksichtigen, um langfristig eigene Schwächen, Erfahrungsmängel und Rationalitätsdefizite zu überwinden. Dabei werden diese Lernprozesse als gestaltbare Instrumente einer Unternehmensführung gesehen, deren Zweck vor allem in der Überwindung der Abhängigkeit der Organisation von personalisiertem Wissen bzw. in der Verbesserung der Fähigkeit zu „systemischem Denken“ liegt (vgl. Elkjaer, 1999; Poell, 2000). Zu den Ansätzen, die auf einer solchen Forschungstradition basieren, die sich einem funktionalistisch geprägten Paradigma verordnet sieht, gehören unter anderem Ansätze der präskriptiven Schule (vgl. insbesondere Garratt, 1987; Pedler/ Burgoyne/Boydell, 1991; Senge, 1991) und präskriptiv orientierte deskriptive Modelle (vgl. u.a. Argyris/Schön, 1978; Duncan/Weiss,1979; Kim, 1993).

Dem gegenüber stehen Ansätze, die den sozialen Prozess des Lernens in den Mittelpunkt der Analyse rücken und die funktional orientierten Forschungsrichtungen kritisieren (vgl. bspw. Brown/Duguid, 1991; Blackler, 1995; Weick/Roberts, 1996; Gherardi, 1996). Dabei wird organisationales Lernen nicht als eindimensionales funktionales Konzept zur Lösung und Bewältigung von organisationalen Problemen gesehen, sondern vielmehr wird versucht, die individuelle Sinngebung („sense-making“) besser zu verstehen, wodurch die Lernprozesse im jeweiligen sozialen Kontext rekonstruiert werden. Erfahrungen und damit einhergehende Gedankenkonstrukte, auf deren Basis organisationales Lernen stattfinden kann, entstehen durch soziale Interaktion, normalerweise eingebettet in dem Setting des Arbeitsplatzes. Lernen wird zu einem sozialen Konstrukt und wird als ein politischer Prozess, der in einer Unternehmenskultur impliziert ist, betrachtet (Easterby-Smith/Araujo, 1999). 
"Organizations, through actions of those in charge, construe their identity by transforming change, past choices, past experiments, inventions and so on, into rational accounts of knowledge. In such a way, they symbolically shape the organization, transforming knowledge into the web of experiential constraints that members perceive as the objective aspect of the organization (that is, the constitutive order which transcends the individual power to act)" (Nicolini/Meznar, 1995, S. 740-741, in: Easterby-Smith/Araujo, 1999).

In den kognitivistischen Lerntheorien rückt der Mittelpunkt der Betrachtungen auf das Lernen selbst und die kognitiven Strukturen sozialer Systeme. So beschäftigen sich kognitive Lerntheorien mit der Frage, in welcher Weise der Mensch Wissen aufbaut, neue Erfahrungen mit bestehendem Wissen integriert, wie Wissen im Gedächtnis organisiert ist und für Problemlösungen wieder aktiviert werden kann (vgl. Wiegand, 1996). Die Black Box „Individuum" wird zu erhellen versucht, indem der Fokus weg von sichtbaren Verhaltensänderungen auf kognitive Strukturen gelegt wird.

Es gilt individuelle Lernergebnisse in kollektive Handlungstheorien einfließen zu lassen und dort zu verankern (Argyris/Schön, 1978; DuncanMeiss, 1978). Dabei werden Organisationen als soziale intelligente Systeme begriffen, die die Fähigkeit besitzen, im Laufe der Zeit Erfahrungen zu sammeln, zu speichern und weiterzuverarbeiten. Die Lernprozesse in den basalen kognitiven Tiefenstrukturen finden ihren beobachtbaren Ausdruck im Verhalten bzw. in der Oberflächenstruktur der Organisation und prägen gewissermaßen die eigenständige Identität einer Organisation (vgl. Schüppel, 1996, S. 15, in: Neumann, 2000, S. 78-79).

Eine Verknüpfung zwischen organisationalem Lernen und Wissensmanagement kann man insofern herstellen, indem man seine Ausführungen bspw. auf der Tatsache gründet (vgl. Wiegand, 1996; Wiegand/Roehl, 1998), dass organisationales Lernen einen Prozess darstellt, der im Wechselspiel zwischen Individuen und Organisationen abläuft. Dabei erwerben Organisationsmitglieder ihr Wissen und Informationen über die Beziehungen der Oraanisation zu ihrer rele- 
vanten Umwelt, wodurch dieses legitimiert und kommuniziert werden kann, sodass Lernprozesse letztlich einen wesentlichen Beitrag zur Überlebensfähigkeit der Organisation liefern (Neumann, 2000). Es werden verschiedenste Lernniveaus festgestellt: bei Argyris/Schön (1978) beispielsweise das Single-LoopLearning, Double-Loop-Learning und Deutero-Learning und bei Senge (1990) findet man beispielsweise Practice-Learning, Principle-Learning sowie EssenceLearning.

Beispielhaft für die Betrachtung von Wissensmanagement in Form von organisationalem Lernen soll das Konzept von Pawlowsky (1994) kurz dargestellt werden. Lernprozesse, die einerseits von Individuen durchlaufen und andererseits von diesen auf organisationaler Ebene intendiert werden, bilden den Kern und Ausgangspunkt seines Wissensmanagement-Ansatzes. Es gilt, die Qualifikationsanforderungen an ein Unternehmen zu erfüllen und Strategien umsetzen zu helfen bzw. strategiegestaltend wirksam zu werden, was durch betriebliche Weiterbildungsmaßnahmen, die den Kernpunkt eines Wissensmanagement-Konzepts ausmachen, erreicht werden soll.

Unternehmen werden als „vergegenständlichte Wissenssysteme (...), in denen in Wechselwirkung individuelles und organisationales Lernen stattfindet" (Pawlowsky, 1992, S. 199) verstanden. Unterschiedliche Lernebenen (beispielsweise Individuum, Team/Gruppe, Organisation, Netzwerk), verschiedene Lernformen (kognitives Wissen, Kultur und Verhalten), Lerntypen, wie sie auch bei Argyris/ Schön (1978) zu finden sind (Single-Loop, Double-Loop und Deutero-Learning) sowie Lernphasen (Identifikation, Diffusion, Modifikation, Aktion) (vgl. Pawlowsky, 1992; 1994) werden konstatiert.

Dieses konzeptionelle Rahmenmodell liefert Ansatzpunkte für eine Wissensdiagnostik und dient als Ausgangspunkt für kontextuelle Gestaltungsüberlegungen eines Wissensmanagements, indem es eine lerntheoretisch fundierte Checkliste für lernrelevante Strukturen und Prozesse innerhalb der Organisation bietet (Neumann, 2000). 
Der Forschungsbereich des organisationalen Lernens wurde in den letzten Jahren zu einem Allheilmittel für Problemstellungen im Bereich der Organisationsforschung hochstilisiert. Andererseits wurde das, was unter organisationalem Lernen zu verstehen ist, in einer großen Anzahl von Ansätzen, Modellen und Theorien immer weiter ausdifferenziert, ohne eine einheitliche Grundlage zu schaffen. Drei Hauptkritiklinien sollen im Folgenden festgehalten werden. Diese Kritik an organisationalem Lernen gilt als konzeptionelle Grundlage für Wissensmanagement und ist daher überwindbar (vgl. Roehl, 2000):

(a) die positiv assoziierte Analogiebildung zum individuellen Lernen

Ein Lernprozess wird als erfolgreich identifiziert, wenn ein Individuum in einer Situation ein besseres, sinnvolleres bzw. effizienteres Verhalten demonstrieren kann (Weick, 1991), als es bisher in einer ähnlichen Situation gezeigt hat. Es wird hierbei auf der Grundannahme aufgebaut, dass sich Individuen fortwährend ähnlichen Situationen gegenübersehen. Wird dies auf Organisationen übertragen, dann müsste man theoretisch auch für Unternehmen von stabilen und gleich bleibenden Situationen ausgehen. Jedoch auf Grund der Tatsache, dass sich Organisationen turbulenten Umwelten gegenübersehen, ist ein solches Verständnis von Lernen als problematisch zu bewerten (vgl. kritisch auch Berggren, 1994). Doch bereits die Grundannahme an sich sollte hinterfragt werden; auch Individuen finden sich in turbulenten Umwelten wieder, sodass der erste Kritikpunkt in diesem Ausmaß nicht übernommen werden sollte.

(b) eine mangelnde empirische Fundierung organisationalen Lernens

Es existieren zwar Forschungsergebnisse, jedoch werden Forschungsprojekte oftmals auf Grund positiver Konnotationen von Lernen mit dem Label "organisationales Lernen" versehen und liefern keine Beiträge dazu, organisationale Lernprozesse besser zu verstehen. Auch Huber (1991) schlägt diesen Punkt als Kritik für eine Diskussion vor, dass sich keine kumulative und empirische Forschung auf diesem Gebiet findet, die tatsächlich die Effizienz und Effektivität der Lernprozesse in den Vordergrund stellt. 
(c) das Problem genereller Gestaltungsmaßnahmen im organisationalen Lernen Organisationspraktisch betrachtet erlangt organisationales Lernen hohe Relevanz, wobei jedoch nicht nachgewiesen werden kann, ob organisationstheoretisches und damit abstrakt-konzeptionelles Wissen in die Organisationspraxis diffundieren kann. Es ist in Frage zu stellen, ob praktische Gestaltungsempfehlungen abgegeben werden können, da ja nicht davon ausgegangen werden kann, dass es ein "richtiges" oder "effizientes" organisationales Lernen gibt, was jedoch durch die Unternehmenspraxis durch die Verwendung dieses Terminus auch in Verbindung mit dem Begriff der "lernenden Organisation" suggeriert wird.

Weitere Kritik an den Ansätzen des organisationalen Lernens findet sich beispielsweise bei Romhardt (1995), Huber (1991) oder North (2002). 


\section{Wissensmanagement haben}

\section{1. Überblick}

Die Bandbreite an Lösungsansätzen in einem funktionalistischen Paradigma umschließt Ansätze, die sich an positivistische Annahmen anlehnen, wie sie einerseits im Rahmen der Informatikwissenschaften entwickelt wurden (vgl. Prusak, 1998; Faisst, 1996, in: Neumann, 2000; Roehl, 2000; Boland/Tankasi, 1995), andererseits Ansätze, die sich in einem Spannungsfeld zwischen Annahmen aus einem funktionalistischen und denen aus einem interpretativen Paradigma wieder finden.

Allen Vertretern dieser Logik ist gemein, dass sie eine objektive Realität als gegeben annehmen, auch wenn in der einen starken oder abgeschwächten Form, und auch von der Grundannahme ausgehen, dass es Unternehmen möglich ist, individuelles Wissen auf eine organisationale Ebene zu transferieren und Wissen managebar ist. Auch wird der Praktikabilität der Konzepte eine Beachtung geschenkt und es werden ökonomisch verwertbare Lösungen von Wissensmanagement ausgearbeitet. Dabei kommt vor allem dem Teilbereich Management eine bedeutende Rolle zu, da vor allem durch dieses das Verhalten der Mitarbeiter und somit der Verlauf des Unternehmens geplant und gesteuert werden kann. So wird bei der Entwicklung von Wissensmanagement-Konzepten auf deren Umsetzung bedacht genommen, um die grundsätzlich vertretene Praktikabilität der Modelle zu gewährleisten.

Die sieben in weiterer Folge diskutierten Ansätze umfassen „die Wissensspirale“ von Nonaka/Takeuchi (bspw. 1995; 1997; 2000), den Ansatz der „K-Wörter" von DavenportPrusak (1998), den Ansatz des "ganzheitlichen Wissensmanagements" von Albrecht (1997), die „acht Bausteine des Wissensmanagements" von Probst/Raub/Romhardth (1997), „die Wissenstreppe“ von North (2002), „das Schichtenmodell" von Pautzke (1989) sowie den Ansatz "das Lebenszyklusmodell des Wissensmanagements" von Rehäuser/Krcmar (1996). Diese Ansätze zeigen die innerhalb dieses Paradigmas bestehende Vielfältigkeit auf. 
So gehen Nonaka/Takeuchi (1995) davon aus, dass der Schwerpunkt im Rahmen eines Wissensmanagement-Konzepts auf das Schaffen von Wissen ( ${ }_{n}$ knowledge creation") gelegt werden soll. Dabei werden in einem Unternehmen zwei Wissensarten festgestellt ("implizites und explizites Wissen"), die sich auf einer individuellen und organisationalen Unternehmensebene entfalten. Wissensschaffung und -umwandlung erfolgen in einem wissensbasierten Unternehmen dann im Rahmen spiralförmig ablaufender Prozesse ("Wissensspirale") durch Interaktion und Verbindung der beiden Wissensarten und Unternehmensebenen.

Denselben Prozess wie Nonaka/Takeuchi in den Mittelpunkt stellend betrachten auch Davenport/Prusak (1998) die Wissensgenerierung als grundlegenden Prozess eines Unternehmens, wo es das in Routinen und Praktiken eingebettete Wissen zu ergründen gilt. Hierzu werden die in einem Unternehmen stattfindenden Austauschbeziehungen von dem Gesichtspunkt der "Wissensmärkte" aus untersucht. Vier Situationsbeschreibungen („K-Wörter") definieren den Umwandlungsprozess.

Ein "ganzheitliches Wissensmanagement" wird von Albrecht (1997) begründet, der es damit schafft, den Fokus auf das Personal und die technische Umsetzung von Wissensmanagement zu überwinden. Im Rahmen von drei Gestaltungsdimensionen - dem Wissensressourcen-Management, Human-RessourcenManagement sowie Wissenstechnik-Management - werden auf den einzelnen Managementebenen Verantwortlichkeiten festgesetzt.

Das Unternehmen in Kernprozesse teilend und Wissensmanagement mittels prozessorientierten Bausteinen aufbauend, stellt sich das Konzept von Probst/ Raub/Romhardth (1997) dar. Durch diese Strukturierung eines Unternehmens werden Wissensbestände identifiziert und versucht, das nötige und relevante Wissen zum richtigen Zeitpunkt an den richtigen Ort für die richtigen Leute zugänglich zu machen. 
Auf eine andere Art geht North (2002) an die Definierung eines Wissensmanagement-Systems heran. Auch von einer Trennung der Termini Daten, Informationen, Wissen, usw. den Ausgang nehmend entwickelt er eine "Wissenstreppe", deren Stufen von einer wissensorientierten Unternehmensführung gestaltet werden sollen. Drei Handlungsfelder, die durch das strategische und operative Wissensmanagement sowie ein Informations- und Datenmanagement gebildet werden, stellen das Fundament eines Wissensmanagements dar. Über Wissensmärkte (vgl. Davenport/Prusak, 1998) wird eine organisationale Neuausrichtung verfolgt, indem eine Wissensorganisation etabliert wird.

Pautzke (1989) entwickelt im Rahmen seines Konzepts das sog. „Schichtenmodell", wobei er versucht, die einzelnen Bereiche von Wissen und einer organisationalen Wissensbasis zu definieren. Dabei kommt es vor allem zu der Ergründung des „im Prinzip verfügbaren Wissens“ und der kollektiven Wissensbasis, die es für Unternehmen zu erschließen gilt.

In dem von Rehäuser/Krcmar (1996) entwickelten Wissensmanagement-Konzept finden sich bereits bekannte Erklärungsansätze (vgl. bspw. die Ausführungen von Pautzke, 1989 und Probst et al., 1997). Wissensmanagement als eine Querschnittsfunktion eines Unternehmens betrachtend, wird der Führung die Aufgabe zugesprochen, den Wissensbedarf festzustellen, der von unteren Managementebenen mittels personeller, organisatorischer und technischer Ressourcen gedeckt wird. In Form eines "Lebenszyklusmodells" werden die genauen Aufgaben eines Wissensmanagements definiert. 


\subsection{Die Wissensspirale}

Als Anfang der neunziger Jahre eine Publikationsflut rund um den Faktor Wissen begann, setzten sich auch die Autoren Nonaka und Takeuchi mit dieser Thematik auseinander. Der Fokus wurde jedoch nicht ausschließlich und begrenzend auf den Faktor Wissen, sondern viel mehr auf die Schaffung desselbigen ("knowledge creation") gelegt. Nicht nur existierendes Wissen soll erworben, angesammelt und verwendet, sondern neues Wissen geschaffen werden. Faktoren, die japanische Unternehmen erfolgreich agieren lassen, werden somit einerseits in der „knowledge creation“ innerhalb eines Unternehmens und andererseits in der Nutzung von externem Wissen, das dann in ein dem Unternehmen zugängliches internes Wissen umgewandelt wird, und somit die organisationale Wissensbasis erweitert und bereichert, gesehen. Nur dadurch kann eine ständige Innovationspolitik verfolgt und als Quelle des Erfolgs genutzt werden (Nonaka/Takeuchi, 1995).

Dieser Wissensmanagement-Ansatz zeigt die Kontroversen zwischen einem japanischen und einem westlichen Verständnis hinsichtlich von Wissen und Wissensmanagement auf und bewirkte, dass die akademische Welt für unternehmensinterne und -externe wissensschaffende Prozesse sensibilisiert wurde.

Laut Nonaka/Takeuchi (1994) divergiert das Verständnis von Wissen zwischen westlicher und japanischer Kultur. In ersterer wird dieser sehr schwer fassbare abstrakte Begriff "Wissen" in Form von "hard facts" zu verstehen versucht und wird demnach etwas Formalem, Systematischem und Explizitem angeglichen. In solchen Konzepten findet man - wie bereits erwähnt - die Termini "Wissen", „Informationen" und "Daten" oftmals aus-tauschbar vor. Japanische Unternehmen hingegen legen ihren Schwerpunkt auf das implizite Wissen.

Diese beiden Betrachtungsweisen werden an die Dichotomie Polanyis (1962) angelehnt. Somit wird das Forschungsfeld des Wissensmanagements von zwei Arten von Wissen dominiert, nämlich durch das explizite Wissen („explicit know- 
ledge") sowie das implizite Wissen ("tacit knowledge"). Ersteres schließt Formales ein, das sich beispielsweise in Handbüchern, technischen Daten und mathematischen Formeln wiederfindet. Wissen kann somit in dieser kodifizierten Form zwischen Menschen transferiert werden. Im Gegensatz dazu steht das implizite Wissen, das dadurch gekennzeichnet ist, dass es sich dem formalen Ausdruck entzieht. Dieses Wissen basiert auf den individuellen Erfahrungsschätzen und inkludiert persönliche Überzeugungen, Perspektiven und Wertsysteme. Die gängige epistemologische Ausrichtung, in der das erkennende Subjekt vom Objekt der Wahrnehmung getrennt wird, wird überwunden und durch die Selbstbeteiligung und das Engagement eines Einzelnen wird Wissen generiert. Das KnowHow, handwerkliche Geschick und Fertigkeiten sowie mentale Modelle (Bilder von der Realität und Zukunft "was ist" und "was sein sollte") werden unter dem Begriff "implizites Wissen" subsumiert (Polanyi, 1962). Wissen kann im Rahmen dieses Ansatzes von Nonaka/Takeuchi lediglich dann geschaffen und erweitert werden, wenn Wissen zwischen beiden Bereichen, auch "Wissensumwandlung" genannt, interagiert. Denn das menschliche Erkennen wird als ein deduktiver Prozess einzelner gesehen, die in ihrer Wahrnehmung nie von der sozialen Interaktion abgeschnitten sind (Nonaka/Takeuchi, 1996).

Die komplementäre Beziehung zwischen den beiden Wissensarten kommt auf verschiedenen Ebenen zum Ausdruck, nämlich der individuellen, der Gruppensowie der Unternehmensebene. Dadurch kann gezeigt werden, dass der Einzelne durch sein Handeln mit dem Unternehmen verflochten ist und das eigene Wissen in das organisationale Wissensnetzwerk durch zwangsläufig in einem Unternehmen stattfindende Interaktionen einfließen lässt (Nonaka/Takeuchi, 1995).

Ein wissensbasiertes Unternehmen ("knowledge-organisation") definiert sich über seine Fähigkeit, sich an die sich ändernde Umwelt anzupassen, indem es neues Wissen schafft, dieses effektiv weitergibt und in Praktiken (Handlungen) einbettet. Das einzelne Individuum muss sich in einer Atmosphäre und einem Kontext wieder finden, in der bzw. in dem es sich weiterentwickeln kann und Kreativität 
gefördert wird. Somit wird jedem einzelnen Mitarbeiter zugesprochen, dass er seine Vorstellungen über Realitätsinhalte kundtun kann. Dieser dynamische Vorgang unterliegt einem fortwährenden Prozess, der Wissen erst generieren lässt. Dieser Zugang, der vor allem die Person in den Mittelpunkt stellt, bedingt, dass Unternehmen nicht mehr ausschließlich ihre Aufmerksamkeit und Energie auf Daten und auf die Organisation von Informationsverarbeitung fokussieren, sondern einem gemeinsamen Verständnis der Werte und Ziele im Unternehmen eine größere Bedeutung zusprechen (Nonaka/Takeuchi, 1994; 1995).

Die Wissensschaffung setzt sich wie oben bereits skizziert aus zwei Hauptprozessen zusammen: der Interaktion und komplementären Beziehung zwischen den beiden Wissensarten sowie den Wissensebenen, auf denen Wissen geschaffen wird. Es werden dadurch vier Formen der Wissensumwandlung erschlossen, was in folgender Form dargestellt werden kann:

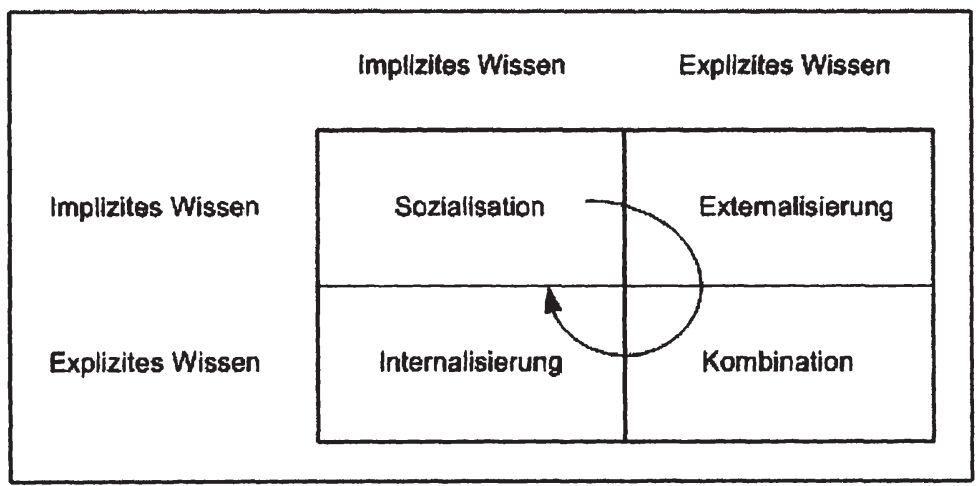

Abbildung 5: Vier Formen der Wissensumwandlung (Nonaka/Takeuchi, 1995)

Im Rahmen der Sozialisation kommt es zu einem Aneignen von Wissen durch ein Individuum. Darauf folgend artikuliert es das Wissen durch Externalisierung und schafft damit eine Transparenz, wodurch das ihm eigen gewesene Wissen zu einem allgemein zugänglichen und somit expliziten Wissen transferiert wird. Wird das externalisierte Wissen durch andere Individuen, die oftmals eine Gruppe bilden, aufgenommen und typisiert, wird dieser Vorgang als Kombination bezeich- 
den, aufgenommen und typisiert, wird dieser Vorgang als Kombination bezeichnet. Die Erfahrungen, die im Rahmen der Kombination mit einfließen, festigen in Form des Prozesses der Internalisierung das implizite Wissen der einzelnen AKteure und lassen diese Vierfeldermatrix wieder von neuem, jedoch auf einer höheren Wissensebene starten.

Diese Wissensumwandlung und -schaffung erfolgt spiralenförmig. Diese Prozesse werden daher zusammenfassend auch als die "Wissensspirale" bezeichnet. Das personengebundene Wissen wird dem Unternehmen zugänglich und explizit gemacht, was über die Erweiterung der organisationalen Wissensbasis den Kreislauf schließend den Wissensbestand des Individuums selbst schlussendlich bereichert und einen neuen Prozess intendiert, dieses in einer Person auf höherer Wissensebene gefestigte Wissen zu artikulieren (Nonaka/Takeuchi, 1994; 1995).

Das kartesianische Verständnis von der Aufspaltung in das Erkennende und Erkannte überwindend wird der Fokus in diesem Konzept auf die Generierung organisationalen Wissens gelegt. Nonaka/Takeuchi gehen von der Grundannahme aus, dass Wissen lediglich von Einzelpersonen geschaffen werden kann und somit auch Organisationen ohne einzelne kein Wissen erzeugen können (Nonaka/Takeuchi, 1996, S. 71). Die ontologische Dimension von Wissen und damit einhergehende individuumszentrierte und subjektive Sichtweise öffnet ein Kritikpotential, das beispielsweise von Schreinemakers/Essers/ Schreyögg (1997) aufgegriffen wird.

Organisationales Lernen wird auf die Wissensaneignung von und die Wissensvermittlung zwischen Individuen zurückgeführt. Neues Wissen hat seinen Ursprung immer im impliziten Wissen von Personen und wird durch Artikulation und Kommunikation explizit. Alleine die Zuordnung des Ursachepunkts der Wissensgenerierung ist Gegenstand von kritischen Überlegungen. Schreyögg (1997) sieht neues Wissen ausschließlich aufbauend auf einer organisationalen Wissensbasis entstehen. Darüber hinaus kann nach seinem Ermessen Wissen auch Sarah Meisenberger - 978-3-631-75453-5 
geschaffen werden, wenn nicht alle vier Phasen der Wissensspirale durchlaufen werden. Beide Kritikpunkte relativierend betont Capurro (1998), dass bei Betrachtung des Spiralmodells zwar die Schlussfolgerung gezogen werden kann, dass im Rahmen des Konzepts von Nonaka/Takeuchi $(1994 ;$ 1995) behauptet wird, Wissen müsse vom Individuum ausgehen. Dennoch könnten alle vier Stadien der Wissensumwandlung als Startpunkt gewählt werden (Capurro, 1998). Aus den Ausführungen von Nonaka/Takeuchi $(1994 ; 1995)$ geht seiner Ansicht nach nicht hervor, dass alle vier Phasen der Wissensspirale durchlaufen werden müssen, damit es zu einer Wissensgenerierung kommen kann (Capurro, 1998).

Die Ausrichtung dieses Konzepts an den Unternehmenszielen lässt eine gesamtunternehmerische Ausrichtung erkennen und die Förderung der Kreativität schließt eine Kontextgestaltung mit ein. Der Managementprozess selbst findet jedoch keine Berücksichtigung, sondern "Managen" unterliegt eher dem Zufall, als dass es planerisch gestaltet werden könnte. Die Schlüsselrolle im Rahmen eines Wissensmanagements wird in diesem Ansatz, wenn, dann nur dem Mittelmanagement zugesprochen, das als Knotenpunkt, der die Verbindung zwischen Unternehmensführung und Basis herstellt, als Brücke zwischen den visionären Idealen der Spitze und der oft chaotischen Arbeitsrealität, fungieren soll. Eine solche Betrachtungsweise von Organisationen gleicht einer maschinellen Steuerungslogik, die für die vorliegende Arbeit nicht übernommen werden wird.

Die in diesem Ansatz durchgeführte Fokussierung auf Wissensschaffung und umwandlung innerhalb von Unternehmen wird für die vorliegende Arbeit nicht übernommen. Die Bedeutung von innovativen Ideen und Innovationsprozessen wird nicht als gering eingeschätzt, jedoch wird angezweifelt, dass der Schwerpunkt eines Wissensmanagements auf den Bereich "Innovation" gelegt werden soll, um gegenüber seinen Wettbewerbern einen komparativen Vorteil zu generieren. Es ist ein anzustrebender Effekt von Wissensmanagement, jedoch sollte ein Wissensmanagement ein Instrument für das Management darstellen und somit Lösungsvorschläge bieten, wie das Management bestehende Ressourcen besser planen, koordinieren und folglich managen kann. Zwar bietet die Wis- 
sensspirale an sich einen guten Überblick über einen möglichen Ablauf der "knowledge creation", jedoch wird in der vorliegenden Arbeit dem Management eine bedeutendere Rolle zugesprochen, die sich in dem Ansatz von Nonaka/Takeuchi nicht findet. Zusätzlich dazu wird angezweifelt, dass durch das Durchlaufen der Wissensspirale gewährleistet werden kann, dass innovative 1deen entstehen, die sich dann auch am Markt gegenüber der Konkurrenz durchsetzen. Dies ist ein Anspruch des Ansatzes, der zwar eintreten kann, jedoch nicht unbedingt muss.

Nonaka/Takeuchi legen eine Trennung von implizitem wie explizitem Wissen zugrunde, und zeigen, dass diese beiden Wissenskategorien in einem dynamischen Wechselverhältnis und Austausch bestehen müssen. Die Wissensspirale an sich ist idealtypisch gezeichnet und es wird angezweifelt, ob sie in dieser Form in Unternehmen überhaupt eine Anwendung finden kann, was unter Umständen auch die mangelnden Ausführungen der Implementierung dieses Ansatzes begründet.

Eine empirische Analyse der Produkt- und Prozessentwicklung erfolgte in japanischen Unternehmen und findet sich auch in Form von Fallstudien in den Werken von Nonaka/Takeuchi (beispielsweise 1995). 


\subsection{K-Wörter}

Der von Davenport/Prusak (1998) entwickelte Ansatz legt den Fokus der Betrachtung auf ein ganz bestimmtes Phänomen einer innerhalb der Black Box "Unternehmen" ablaufenden Dynamik. Es gilt, das in Routinen und Praktiken eingebettete Wissen zu ergründen, das ein Unternehmen in nützliche Produkte und Dienstleistungen umsetzt. Dadurch soll überwunden werden, dass die Analyse von Unternehmen dahingehend beschränkt wird zu untersuchen, welche Ressourcen investiert (in das Unternehmen hineingehen) und welche Produkte auf welchen Märkten vertrieben wurden (aus dem Unternehmen hinausgehen).

Die Aufmerksamkeit dieses Ansatzes wird auf die Mitarbeiter gelenkt, denn das, was diese wissen, steht im Mittelpunkt aller organisationalen Funktionsabläufe. Somit wird das Grundlegende eines Unternehmens - die Mitarbeiter - zu ergründen versucht, was sich auf einen irreduziblen und für Performance, Produktivität und Innovation entscheidenden Wettbewerbs-vorsprung auswirkt (Davenport/Prusak, 1998).

Der Wissensbegriff wird hierbei weit gefasst und eine Abgrenzung aus pragmatischen Gründen nur zu den Termini "Daten" und „Informationen" zu ziehen versucht. Somit versteht sich in diesem Konzept Wissen als

„eine fließende Mischung aus strukturierten Erfahrungen, Wertvorstellungen, Kontextinformationen und Fachkenntnissen, die in ihrer Gesamtheit einen Strukturrahmen zur Beurteilung und Eingliederung neuer Erfahrungen und Informationen bietet. In Organisationen ist Wissen häufig nicht nur in Dokumenten oder Speichern enthalten, sondern erfährt auch eine allmähliche Einbettung in organisatorische Routinen, Prozesse, Praktiken und Normen" (Davenport/Prusak, 1998, dt. Übersetzung, S. 32).

Wissen ist zum Teil fließend, zum Teil aber auch formell strukturiert; es kann intuitiv sein und ist schwer in Worte zu fassen. Dem Konzept Wissen werden auch 
übergeordnete Begriffe wie "Weisheit" und "Einsicht" zugeordnet. Es lässt sich klar erkennen, dass Wissen keineswegs wohlgeordnet oder einfach zu erfassen ist, da es sich eben aus den verschiedensten Elementen zusammensetzt.

Potentiell stehen Wissensbestände den einzelnen Unternehmen alleine durch die Mitarbeiter zur Verfügung. Doch der Weg scheint teilweise zu verstrickt und nur schwer begehbar zu sein, wenn es darum geht, das individuelle Wissen zu einem kollektiv Gewussten zu transferieren. Betrachtet man jedoch die in einem Unternehmen stattfindenden Austauschbeziehungen von dem Gesichtspunkt eines Marktes, so lassen sich gewisse Mechanismen erkennen, die diesen Umwandlungsprozess erleichtern helfen. Wissensmärkte gilt es auszumachen, um Transaktionen innerhalb eines Unternehmens sowie mit externen Partnern effizienter gestalten zu können. Hierbei finden sich Verkäufer wie Käufer von Wissen - auf der einen Seite Leute, die Probleme und Fragestellungen zu lösen haben, dies aber mit ihrem eigenen Wissensbestand nicht bewältigen können, und auf der anderen Seite Leute, die über ein Wissen verfügen, das in diversen Bereichen einzigartig ist, die daher als Experten auftreten können und die im Unternehmen als solche zu Rate gezogen werden. Aspekte wie Gegenseitigkeit, Ansehen und Selbstlosigkeit, die in eine Unternehmenskultur des Vertrauens eingebettet sind, erfahren eine besondere Berücksichtigung (Davenport/Prusak, 1998).

Wissensgenerierung kann innerhalb von Unternehmen durch die Umwandlung von Daten und Informationen vonstatten gehen; einem Prozess, der durch vier „K"- Wörter beschrieben werden kann:

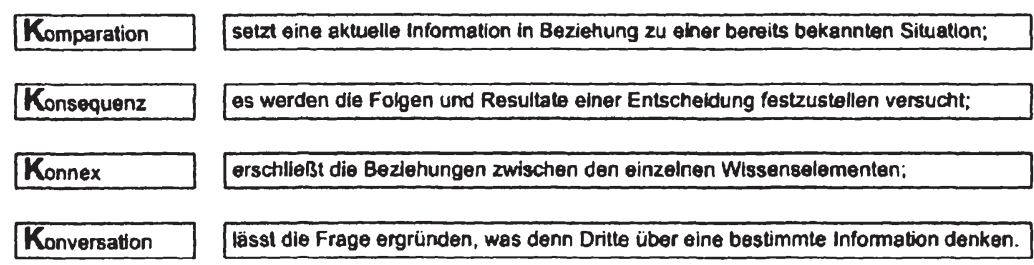

Abbildung 6: K-Wörter des Umwandlungsprozesses (Davenport/Prusak, 1998) 
Durch die Etablierung eigener Wissensabteilungen (Forschungs- und Entwicklungsabteilungen) oder durch innovative Adaptionen, mit deren Hilfe Unternehmen ihre Routineaktivitäten ändern, um sich neuen Herausforderungen anzupassen, kommt es zu einer Erweiterung des Organisationswissens. Andererseits kann eine Wissensvermehrung auch durch das Nutzbarmachen von externen Ressourcen erfolgen. So wird beispielsweise bei Kauf oder Fusionen von Unternehmen oder bei der Beschaffung von "Mietwissen" der organisationale Wissensbestand vermehrt.

Das Ansammeln von Wissen schließt jedoch nicht zwangsläufig auch das nutzbringende Einsetzen dieses Faktors mit ein. Es muss das Augenmerk auch darauf gelegt werden, wie man den organisationalen Wissensbestand kodifiziert, da er Mitarbeitern verständlich gemacht werden muss. Dies kann durch eine Kartographisierung von Wissen erfolgen, beispielsweise in Form von Wissenskarten, Gelben Seiten oder vergleichbaren Instrumenten. Man ermöglicht dadurch, dass kollektiv verfügbares Wissen tatsächlich transferiert und von Mitarbeitern für eine verbesserte Lösung von Aufgabe- und Fragestellungen verwendet wird, was unmittelbar zu effizienteren organisationalen Transaktionen führt (Davenport/ Prusak, 1998).

Die Kernaussage dieses Konzepts, nämlich dass der einzige dauerhafte Wettbewerbsvorteil auf dem Kollektivwissen basiert, über das ein Unternehmen verfügt, auf der Effizienz, mit der es dieses Wissen nutzt, sowie auf der Bereitwilligkeit, mit der es neues Wissen erwirbt und anwendet, zeigt einen breiten Gestaltungsraum. Nicht konkret positionieren sich jedoch Davenport/Prusak (1998) hinsichtlich der Frage, wo Wissen in einem Unternehmen lokalisiert wird und von wo aus eine Wissensgenerierung stattfindet. Zwar heben sie den einzelnen Mitarbeiter hervor, indem sie darauf hinweisen, dass das individuelle Wissen das Fundament sämtlicher organisationaler Funktionsabläufe darstellt. Jedoch lässt sich der Anschein nicht verwehren, dass eine organisationale Wissensbasis, die den kritischen Faktor, der letztendlich über Erfolg oder Misserfolg eines Unternehmens gegenüber seiner Konkurrenz entscheidet, in diesem Ansatz als eine Aufsummie- 
rung individueller Wissensbestände betrachtet wird. Dies lässt sich vor allem dadurch zeigen, dass Wissensgenerierung in diesem Ansatz erschlossen wird, indem sich Unternehmen mit anderen fusionieren, "Mietwissen" anschaffen bzw. Wissen kaufen. Es wird wenig auf die internen Prozesse der Interaktion zwischen den Mitarbeitern eingegangen. Zwar werden „Wissensmärkte“ im Unternehmen ausgemacht und diese Idee des Angebots und der Nachfrage von Wissen mag sich auch als dienlich erweisen, jedoch erfolgen die Ausführungen lediglich in einer etwas oberflächlichen Form.

All die Kritikpunkte an diesem Ansatz mögen unter Umständen darauf zurückzuführen sein, dass Davenport/Prusak ziemlich alles, was gewusst werden kann unter Wissen subsumieren und sich auf eine exakte Wissensdefinition nicht einlassen. Auch erfolgt keine klare Abgrenzung zwischen individueller und organisationaler Ebene und diese werden austauschbar verwendet. So wird die Quelle von Wissen nicht lokalisiert, was den Prozess der Wissensgenerierung einer tieferen Analyse nicht zugänglich macht.

Dem Wechselspiel zwischen Technologien und Wissensgenerierung wird eine besondere Bedeutung beigemessen. Und obwohl die Wichtigkeit von einer technologischen Untermauerung von Wissensmanagement keineswegs zu minder eingeschätzt werden soll und dies auch in dieser Arbeit nicht werden wird, haben bereits Davenport/Prusak auf die Grenzen von Technologien im Rahmen eines Wissensmanagements hingewiesen. Denn das reine Vorhandensein technologischer Einrichtungen garantiert alleine kein Unternehmen, in dem Wissen von jedem Mitarbeiter gerne geteilt wird bzw. in dem Wissensmanagement durchgeführt werden kann. Somit soll auch in der vorliegenden Arbeit das Augenmerk darauf gelegt werden, welche Voraussetzungen müssen in einem Unternehmen vorhanden sein, dass es gewährleistet werden kann, dass Wissensmanagement betrieben wird, worauf Davenport/Prusak in ihrem Hauptwerk (1998) nicht weiter eingehen. 
Es wird in diesem Ansatz vorgeschlagen (und auch mit Fallbeispielen untermauert), dass Unternehmen sog. Wissensabteilungen bzw. Wissensmanager im Unternehmen integrieren. Diese würden die für ein Wissensmanagement erforderliche Infrastruktur entwickeln, einrichten und überwachen, Beziehungen zu externen Anbietern von Informationen und Wissen aushandeln und den erforderlichen Input zum Prozess der Wissenserzeugung und Wissensnutzung im Unternehmen leisten. Auch die Kodifizierung von explizitem Wissen sowie die Bewertung und Verwaltung von Wissen würde dieser Abteilung bzw. diesen Stellen obliegen (Davenport/Prusak, 1998). Sicherlich müssen all die genannten Funktionen im Rahmen von Wissensmanagement eine Berücksichtigung erfahren, jedoch wird angezweifelt, ob dies durch eine zentrale Stelle erfolgen kann bzw. soll. Für die vorliegende Arbeit wird diese Herangehensweise nicht übernommen, da Wissensmanagement in dieser Arbeit eine Querschnittsfunktion zugesprochen wird, die durch eine Zentralisierung von Wissensmanagement nicht erfüllt wird. 


\subsection{Ganzheitliches Wissensmanagement}

Im Rahmen eines "ganzheitlichen Wissensmanagements" erhebt Albrecht (1993) den Anspruch, erstmalig für den deutschsprachigen Raum den Blickwinkel weg von einseitig und zu eng konzipierten Modellen, die den Schwerpunkt lediglich auf die technische Umsetzung von Wissens- oder Datenmanagement setzen, sowie weg von Modellen, die den Fokus ausschließlich auf die Personalkomponente legen, zu rücken.

Wissen avancierte zu einem wichtigen und interessanten betriebswirtschaftlichen Untersuchungsobjekt, das im Vergleich zu anderen Unternehmensressourcen einigen Besonderheiten unterworfen ist. Die Komplexität der Wissensstrukturen in einem Unternehmen lässt die Ressource Wissen nur schwer erfassen, wodurch es über das gesamte Unternehmen verteilt ist. Wird Wissen als Produktionsfaktor identifiziert, gestaltet und gesteuert, so kann Wissensmanagement als Instrumentarium betrachtet werden, das die Ressource Wissen in den Mittelpunkt seiner Betrachtung stellt (Albrecht, 1993).

"Ziel des Wissensmanagements ist es, das im Unternehmen vorhandene Potential an Wissen derart aufeinander abzustimmen, dass ein integriertes unternehmensweites Wissenssystem entsteht, welches eine effiziente gesamt-unternehmerische Wissensverarbeitung im Sinne der Unternehmensziele gewährleistet. Dazu bedarf es vor allem der Gestaltung des gesamten Wissens des Unternehmens unter gleichberechtigtem Einsatz natürlicher wie künstlicher Ressourcen zur Wissensverwaltung und -verarbeitung" (Albrecht, 1993, S. 97).

Dem Management wird in diesem Ansatz eine bedeutende Rolle zugesprochen. Es wird als Inbegriff aller im Gesamtunternehmen notwendigen Gestaltungs- und Steuerungshandlungen zur Zielerreichung gesehen und umfasst die Gesamtheit aller Bestimmungshandlungen, die das zukünftige Verhalten des Gesamtsystems Unternehmen festlegen. „Unternehmen“ werden in dieser Arbeit (Albrecht, 1993) in Anlehnung an Ulrich (1970) als komplexe und offene, ziel- und zweckorientier- 
te soziotechnische Systeme verstanden, die durch spezifische Beziehungen mit der Umwelt verbunden sind. Das "Managen von Wissen" besteht hauptsächlich darin, dem Unternehmen die Eigenschaft eines auch in der Zukunft lebensfähigen Systems zu vermitteln. Ein Unternehmen als ein wissensbasiertes und wissensverarbeitendes System betrachtet, findet die Ressource Wissen in sämtlichen Subsystemen vor. Dieses eigene Subsystem, das Wissenssystem, zeichnet sowohl für die Wissensversorgung des Führungs- als auch des operationellen Systems verantwortlich. Das Wissenssystem selbst unterteilt sich wiederum in die Subsysteme Mensch und Betriebsmittel, wobei vor allem für das System "Computer" als maschinelles Informations- und Wissenssystem Interesse gezeigt wird (Albrecht, 1993).

Im Rahmen dieses Ansatzes wird jedoch kein neuer Managementansatz zu verwirklichen versucht, sondern lediglich die konsequente Zusammenführung verbesserter Informations- und Wissenstechniken mit dem Wissensträger Mensch. Das Konzept des ganzheitlichen Wissensmanagements erhebt bewusst bereits durch seine Bezeichnung den Anspruch, in einem Unternehmen integrierend und koordinierend auf die dort ablaufenden Prozesse zu wirken und die Ressource Wissen in ihrer unternehmensweiten Mehrdimensionalität zu erfassen. Erst wenn Wissensmanagement zu einem integralen Bestandteil der Unternehmensphilosophie und Unternehmensstrategie wird, ist die ganzheitliche wissensorientierte Sichtweise aller Unternehmensaufgaben gewährleistet (Albrecht, 1993).

Mithilfe von drei Gestaltungsdimensionen, die auch den Kern dieses Wissensmanagement-Ansatzes bilden, soll die Ganzheitlichkeit von Wissensmanagement gewährleistet werden; diese bestehen einerseits aus dem WissensressourcenManagement, das die Aufgabe verfolgt, das unternehmensweite Wissenspotential zu managen; weiters aus einem Human-Ressourcen-Management, das für das Management aller mit der personellen Wissensverarbeitung verbundenen Prozesse verantwortlich zeichnet; und schlussendlich aus dem WissenstechnikManagement, das sämtliche technische Komponenten, sei es Hard- oder Software oder sonstige Instrumente der Wissensverarbeitung, umfasst. 
Aufbauend auf den oben erwähnten drei Dimensionen kommt es zu einer klaren Definierung der Rollen und Verantwortlichkeiten der einzelnen Managementebenen in diesem Wissensmanagement-Konzept.

So ist das strategische Management beispielsweise für die Verankerung der unternehmensweiten Wissensorientierung in den Unternehmensgrundsätzen und Leitbildern verantwortlich. Dies wird nur dann erfolgreich etabliert werden können, wenn in einem Unternehmen eine dementsprechende Wissenskultur vorherrscht. Eine solche aufzubauen und zu bewahren, fällt ebenfalls unter die Obhut des strategischen Managements. Das Festlegen und die Entwicklung einer unternehmensweiten Wissensstrategie sowie das strategische Management der Humanressourcen und der Wissenstechnik sind allesamt Bereiche, die dem strategischen Management zugesprochen werden.

Die praktische Umsetzung der strategischen Vorgaben ist Aufgabe des operativen Wissensmanagements, damit ,jegliches in der Wertschöpfungskette notwendige Wissen an der richtigen Stelle der Ablauforganisation zum passenden Zeitpunkt in der erforderlichen Menge und Qualität verfügbar ist" (Albrecht, 1993, S.104).

Dieser Ansatz beruht auf einem rationalistischen Unternehmens- und Managementverständnis, das Organisationen auf eine Art und Weise versteht, dass sie unmittelbar den Gestaltungs- und Steuerungsabsichten des Managements unterworfen sind und dass unter Einsatz "richtiger" Mittel und Methoden zielgerichtete Ergebnisse erreicht werden können. Grundsätzlich ist eine solche theoretische Positionierung, wenn man sich in einem funktionalistischen Paradigma verortet sieht, nicht verwunderlich. Gewisse Aspekte von Wissen erfahren jedoch keine Berücksichtigung, die nicht ohne weiteres außer Acht gelassen werden können. Bspw. werden die Besonderheiten von implizitem Wissen und die Interaktionsprozesse, die im Zuge von Wissensentwicklung, und -transfer durchgeführt werden, nicht erörtert. Wissen als Ressource wird zwar auf der Ebene des Individuums lokalisiert, das durch Unterstützung des Subsystems ${ }_{\text {Caranguter" auf }}$ 
organisationale Ebene transferiert werden kann, jedoch wird das in diesem Ansatz zugrunde gelegte Menschenbild nicht näher erläutert.

Dadurch dass das Augenmerk in diesem Ansatz vor allem auf die Wissenssysteme Mensch und Computer gelegt wird und die Anforderung der Ganzheitlichkeit von Wissensmanagement erfült werden soll, reicht eine getrennte Betrachtung und Optimierung der beiden Wissenssysteme nicht aus. Vielmehr sind sie gemeinsam zu gestalten und aufeinander abzustimmen. Die von Albrecht verwendete Sichtweise von Unternehmen sowie die Betrachtung der Wissenssysteme Mensch etc. wird für diese Arbeit nicht übernommen. Die mangelnde Analyse des Menschen, obwohl er als Quelle von Wissen im Unternehmen ausgemacht wird, soll in der vorliegenden Arbeit nicht wiederholt werden. Auch ist die Betrachtung von Wissen als Ressource sicherlich brauchbar, jedoch lässt sie wie oben bereits erwähnt - Aspekte von Wissen, die sich als für ein Wissensmanagement kritisch erweisen, außer Acht.

Die Ganzheitlichkeit des Ansatzes soll durch das Dreierpaket an Arten von Management, die ein "Wissensressourcen-Management", ein "Human-RessourcenManagement" sowie ein "Wissenstechnik-Management" beinhalten, gewährleistet werden. Dies wird als Versuch gewertet, ein ganzheitliches Wissensmanagement durchzuführen. Die vorliegende Arbeit wird darauf nicht rekurrieren, da Wissensmanagement als nicht dem Management vorbehalten angesehen werden soll. Sicherlich wird vor allem der oberen Führungsebene die Verantwortung übertragen, Wissensmanagement in einem Unternehmen zum Thema zu machen und sämtliche infrastrukturellen, personellen und technischen Voraussetzungen zu bieten, jedoch wird angezweifelt, ob ohne Berücksichtigung der informellen Interaktionsprozesse und Organisationsstrukturen, der Anreiz- und Motivationsstrukturen von Individuen etc. ein Wissensmanagement überhaupt möglich ist.

Albrecht (1993) bietet keine Implementierungsinstrumentarien und so stellt sich dieses Konzept ausschließlich auf einer abstrakten Ebene dar. Auch werden kei- 
ne empirischen Untersuchungen, wie Wissensmanagement anhand dieses Konzepts eingeführt wurde, präsentiert. 


\subsection{Die acht Bausteine des Wissensmanagements}

Ein im deutschsprachigen Raum mit großer Resonanz aufgenommener Ansatz wird von Probst et al. (1997) dargestellt, der das Unternehmen mit Hilfe von voneinander abhängigen Kernprozessen erschließt. Diese konnten durch die Gruppierung der Problemstellungen, denen sich Unternehmen in der Praxis gegenübersehen, gebildet werden. Die Wirkungszusammenhänge der einzelnen Bausteine sind vielfältig. Kommt es in einem Teilbereich des Unternehmens zu einer Änderung, hat dies Auswirkungen auf andere Prozesse und somit weitläufig auf das Unternehmen als Ganzes.

Die Kernelemente dieses Wissensmanagement-Konzepts bilden sog. „Bausteine“ eines Unternehmens, die auf Wissensidentifikation, Wissenserwerb, Wissensentwicklung, einer Wissens(ver)teilung, Wissensnutzung und Wissensbewahrung basieren.

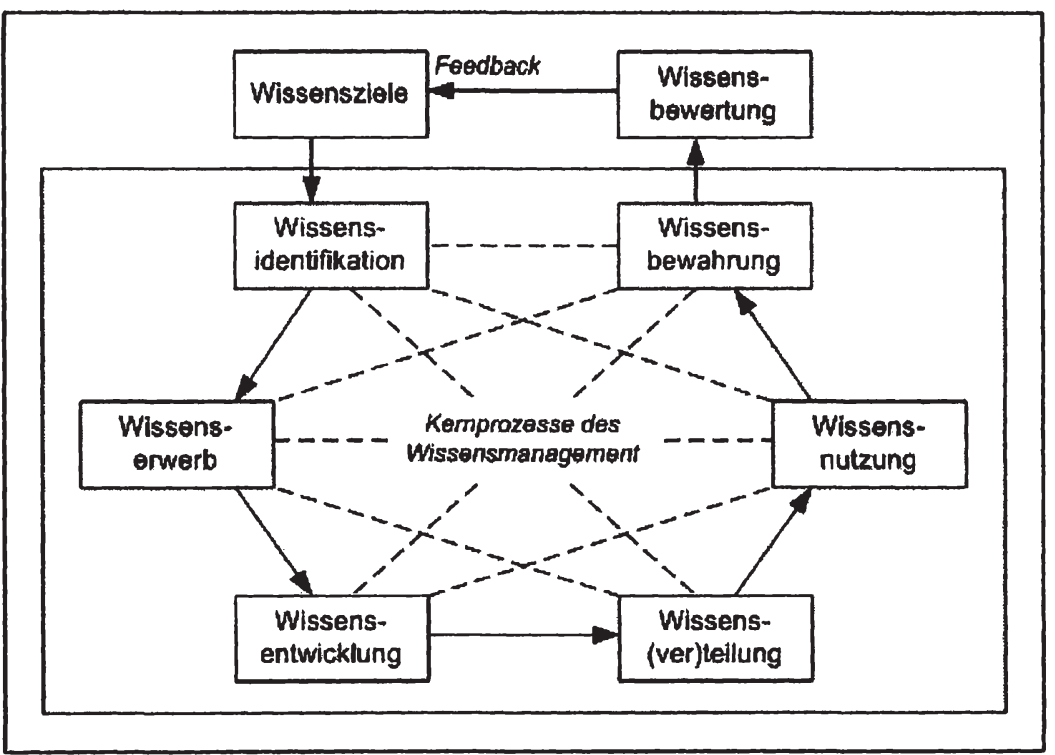

Abbildung 7: Acht Bausteine des Wissensmanagements (Probst et al., 1997) 
Diese Gliederung bietet zwar einen gewissen Überblick, muss jedoch Eingang und eine Einordnung in eine gesamtunternehmerische Planung und Perspektive finden. Somit werden diesem Modell zwei Kriterien hinzugefügt, nämlich:

- Wissensziele und

- Wissensbewertung.

Dadurch wird die Systematik dieses Konzepts an die Logik eines klassischen Managementkreislaufes angelehnt, was sich in der klassischen Managementliteratur immer wieder findet, betrachtet man beispielsweise den Steuerungs- und Regelungskreislauf als Funktionsprinzip kybernetischer Systeme (Probst et al., 1997).

Die Darstellung eines Unternehmens in Teilprozessen setzt eine gewisse Planung voraus, damit eine Organisation als Ganzes auf eine Richtung ausgelenkt und die Teilprozesse untereinander abgestimmt werden können. Dies kann durch die Einbettung des Wissensmanagement-Konzepts auf der Ebene der Unternehmensführung bewerkstelligt werden. So wird dem Management die Aufgabe zugesprochen, auf normativer, strategischer und operativer Ebene Ziele zu definieren, die Klarheit darüber schaffen, was mit einem Wissensmanagement erreicht werden soll und welche Voraussetzungen dafür im gesamten Unternehmen bestehen müssen.

Normative Wissensziele dienen dazu, den unternehmens-politischen und kulturellen Wert von Wissen auf höchster Ebene, nämlich in den Wertvorstellungen und Normen des Unternehmens, festzusetzen. Somit wird großes Augenmerk darauf gelegt, dass die "Philosophie des Wissensmanagements" im Gesamtunternehmen verankert ist und nicht als etwas verkümmert, das nur von einer Ebene oder den oberen Managementebenen getragen wird.

Wie die auf normativer Ebene festgesetzten Wissensziele im Unternehmen umgesetzt werden sollen, wird auf der strategischen und weiters auf der operativen Ebene ausgearbeitet. Die Unterscheidung zwischen diesen beiden deckt sich gänzlich mit der in der Literatur getroffenen (vgl. Gälweiler, 1990), nur dass eben 
Gegenstand der Zieldefinitionen die Ressource Wissen ist. Die Inhalte können mit folgender Grafik veranschaulicht werden:

\begin{tabular}{|c|c|c|c|}
\hline 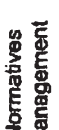 & $\begin{array}{l}\text { Unternehmensverfassung } \\
\text { - rechthlche Strukluren } \\
\text { Auswirkungen auf WM } \\
\text { (Geheimhaltungsregeln etc.) }\end{array}$ & $\begin{array}{l}\text { Untemehmenspolitik } \\
\text { - Wissensleitblld } \\
\text { - Identiflication von krltischen } \\
\text { Wissensfeldem }\end{array}$ & $\begin{array}{l}\text { Untemehmenskultur } \\
\text { - rechtllche Strukturen } \\
\text { Auswirkungen auf WM } \\
\text { (Geheimhaltungsregeln etc.) }\end{array}$ \\
\hline 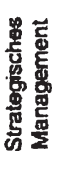 & $\begin{array}{l}\text { Organisatlonsstrukuren } \\
\text { - Konferenzen, Berichtswege, } \\
\text { F\&E-Organlsation. } \\
\text { Erfahrungszirkel } \\
\text { Managementsysteme } \\
\text { - EIS, Lotus-Notes } \\
\end{array}$ & $\begin{array}{l}\text { Progremme } \\
\text { - Kooperation } \\
\text { - Aufbau von Kernkompetenzen } \\
\text { - Informatisierung }\end{array}$ & $\begin{array}{l}\text { Problemverhaiten } \\
\\
\text { - Orientierung von Wissenszielen } \\
\text { - problemorientierte } \\
\text { Wissensidentifizierung }\end{array}$ \\
\hline \multirow[t]{2}{*}{$\begin{array}{l}\text { 总 } \\
\text { 总 } \\
\text { 总 } \\
\text { 总 }\end{array}$} & $\begin{array}{l}\text { Organisatorische Prozesse } \\
\text { - Steuenung von Wissensflüssen } \\
\\
\text { Dispositionsprozesse } \\
\text { - Wissensinfrastruktur } \\
\text { - Wissensbereitstellung } \\
\end{array}$ & $\begin{array}{l}\text { Aurtuäge } \\
\text { - Wissensprojekte } \\
\text { - Aurbau Expertendatenbank } \\
\text { - CBT-Einführung }\end{array}$ & $\begin{array}{l}\text { Leistung- und } \\
\text { Kooperationswerhatten } \\
\text { - Wiesensteilung } \\
\text { - knowledge in action }\end{array}$ \\
\hline & Strukluren & Aktivitäten & Verhalten \\
\hline
\end{tabular}

Abbildung 8: Wissensthemen auf unterschiedlichen Zielebenen (Probst et al. 1977)

Nur wenn ausreichend Transparenz in einem Unternehmen herrscht, können die vorhandenen Wissensbestände ausfindig gemacht werden. Sog. "Wissenskarten“ unterstützen diesen Prozess und es können damit Wissensträger, -quellen, strukturen oder -anwendungen graphisch verzeichnet werden. Ist jedoch der Zugang zu internem Wissen verschlossen, so bietet der Markt Wissensmärkten die Möglichkeit, Wissen von externen Quellen zu erwerben. Beispielsweise können Beziehungen zu Lieferanten, Kunden und Mitbewerbern aktiv gestaltet werden. Unternehmen können neue Mitarbeiter einstellen, Dienst- und Beratungsleistungen beziehen sowie Wissensprodukte (CD-Roms, Studien, etc.) beschaffen. Bevor jedoch externe Wissensbestände teuer zugekauft werden, sollen durch die Prozesse der Wissensentwicklung bereits vorhandene Wissensbestände optimal ausgeschöpft werden und neue Kompetenzen sowie neues Wissen generiert werden.

Ein kritischer Punkt, dem man im Rahmen eines Wissensmanagement-Systems gegenübersteht, ist die Verteilung des Wissens ine eineme Unternehmen5 Wie kुann 
Ein kritischer Punkt, dem man im Rahmen eines Wissensmanagement-Systems gegenübersteht, ist die Verteilung des Wissens in einem Unternehmen. Wie kann es ein Unternehmen schaffen, dass das nötige und relevante Wissen zum richtigen Zeitpunkt am richtigen Ort für die richtigen Leute zugänglich ist? Hierbei wird im Rahmen dieses Ansatzes zwischen zwei Strategien unterschieden. Einerseits werden mit Push-Strategien vorher definierte Wissensbestände auf eine größere Anzahl an Mitarbeitern über bestimmte Wissenskanäle verteilt. Im Gegensatz dazu stehen die Pull-Strategien, mit denen Wissensbestände zwar den Mitarbeitern über interne Informationsnetzwerke verfügbar gemacht werden, es jedoch eine Holschuld bedeutet, sich dieses Wissen im Bedarfsfall auch anzueignen. Insgesamt kommt der Nutzung des organisationalen Wissens eine große Bedeutung zu und indem Aufmerksamkeit auf diesen Prozess gelegt wird, kann eine wirtschaftliche Verwertbarkeit gewährleistet werden. Wissen nutzen muss immer wieder eine Bereicherung darstellen und ein besseres Arbeiten ermöglichen, damit ein Wissensbestand auch in weiterer Folge zur Beantwortung abweichender Fragestellungen herangezogen wird.

Um den organisationalen Wissensbestand für seine Tätigkeiten nutzen zu können, muss das im Unternehmen bzw. den einzelnen Mitarbeitern zugängliche Wissen optimal und in verwertbarer Form abrufbar sein. Fragestellungen, die damit einhergehen, setzen sich mit der Speicherung von Wissen auseinander, um sicherzustellen, dass es zu keinen Verlusten bereits erworbener Wissenspotentiale kommt (Probst et al., 1997).

Durch die acht Bausteine des Wissensmanagements wird eine organisationale Wissensbasis transparent gemacht. Die in diesem Konzept sequentiell ablaufenden Wissensmanagementprozesse werden jedoch in der Praxis als parallel ablaufend eingestuft. Beispielsweise zeigen die Schritte "Wissenserwerb" und "Wissensentwicklung", dass derartige Prozesse eine Darstellung in Sequenzen durchbrechen (Krogh/Nenzin, 1995). Und auf ein Überspringen von Bausteinen bzw. auf ein auf die jeweilige Situation abgestimmtes Ablaufen der Prozesse wird bereits in der von Probst et al. (1997) dargestellten Grafik Acht Bausteine des Wis- 
sensmanagements" mit gestrichelten Linien hingewiesen. Ein selbst verantwortetes Abweichen vom geraden Weg steht somit jedem Unternehmen offen.

Der Schwerpunkt, der in diesem Modell anfänglich explizit auf eine Nutzungsorientierung gelegt wird, lässt sich jedoch nicht in den gesamten Ausführungen nachzeichnen, da erst mit dem Baustein "Wissensnutzung", der den drittletzten Schritt darstellt, die Bedürfnisse der Benutzer in den Vordergrund gerückt werden.

Die Wissenskonzeption in diesem Ansatz rekurriert auf die Dreiteilung zwischen Wissen, Informationen und Daten und Probst et al. sehen es für das Management als unerlässlich an, diese einerseits unterscheiden zu lernen, doch andererseits auch deren Zusammenhänge zu erkennen. Wenn Wissen als Ressource für ein Unternehmen managebar sein soll, reicht es jedoch nicht aus, lediglich die statischen und "harten" Seiten von Wissen festzuhalten. Für die vorliegende Arbeit wird diese Begriffsdefinition somit als zu eng betrachtet. Dem impliziten Wissen sollte eine Bedeutung beigemessen werden, was in diesem Ansatz nicht wiedergefunden werden kann.

Wissen wird im Rahmen dieses Wissensmanagement-Ansatzes in den Köpfen der Mitarbeiter lokalisiert. Aufbauend auf diese Annahme definieren Probst et al. einen Kausalzusammenhang in folgender Hinsicht: „Je höher die Bedeutung organisationalen Wissens für die Wertschöpfung eines Unternehmens ist, um so wichtiger wird auch die intellektuelle Arbeit hochqualifizierten Personals" (Probst et al., 1997).

Dies kann in Unternehmen soweit führen, dass einzelne Schlüsselmitarbeiter zu zentralen und nahezu unersetzlichen Wissensträgern der Organisation werden. Dennoch wird das organisationale Wissen nicht ausschließlich als die Summe des Wissens von Individuen bezeichnet, sondern durch kollektive Wissensbestände ergänzt (Probst et al., 1997). Eine mangelnde Konkretisierung des Begriffs "kollektiver Wissensbestände" lässt eine genaue Definierung von organisati- 
onaler Wissensbasis offen, wodurch diese Idee bzw. Ausführungen für die vorliegende Arbeit nicht übernommen werden. Sog. Wissensarbeiter, die durch ihr hochqualifiziertes Expertentum eine Vorrangstellung im Unternehmen einnehmen bzw. einnehmen können, müssen im Rahmen eines Wissensmanagements zwar berücksichtigt werden, jedoch soll der Fokus dieser Arbeit nicht auf einzelne Mitarbeiter, die durch ihre Ausbildung und ihre "skills" eine besondere Stellung in einem Unternehmen einnehmen, gelegt werden, was hingegen im Ansatz von Probst et al. der Fall ist.

Die Untergliederung von Wissensmanagement in einzelne Bausteine erweist sich als für die Praxis sehr dienlich. So wurden diese auch in Rahmen einer zweijährigen Forschungstätigkeit in Zusammenarbeit mit größeren Unternehmen, die sich zu einem Forum für organisationales Lernen und Wissensmanagement zusammengeschlossen haben, entwickelt. Die acht Bausteine wurden durch weitere zwei erweitert, wodurch gewährleistet wurde, dass auch strategische Elemente eine Berücksichtigung erfahren.

In der vorliegenden Arbeit wird die Annahme getroffen, dass Wissensmanagement nicht lediglich durch das Zusammenspiel diverser Kernprozesse, unabhängig davon, ob es sich um wissensintensive Prozesse handelt, entsteht. Wissensmanagement hat explizites sowie implizites Wissen ebenbürtig zu berücksichtigen und kann die Interaktionsstrukturen innerhalb eines Unternehmens nicht außer Acht lassen. So kommt es im Rahmen dieses Ansatzes durch die Gestaltung von Wissensmanagement in Form von Bausteinen zu einer Komplexitätsreduktion und zu Kausalzusammenhängen, die für die vorliegende Arbeit nicht übernommen werden. 


\subsection{Die Wissenstreppe}

Ein weiterer praxeologischer Zugang zu dem Bereich Wissensmanagement wird durch North (2002) begründet. Die Legitimation für eine verstärkte Auseinandersetzung mit dem Thema Wissen bezieht er aus drei Triebkräften; einerseits durch einen strukturellen Wandel zu einer Informations- und Wissensgesellschaft, ferner durch den vermehrten Einsatz von Informations- und Kommunikationstechnologien und letztendlich durch eine Globalisierung der Wirtschaft, die zu einer internationalen Arbeitsteilung führt. Im Mittelpunkt der Betrachtung steht eine wissensorientierte Unternehmensführung, die imstande ist, zur Verfügung stehende Informationen in Wissen zu transferieren und aus diesem generierten Wissen Wettbewerbsvorteile zu schöpfen. Dabei wird eine organisationale Effizienzsteigerung verfolgt und die Qualität des Wettbewerbs zu verändern versucht.

Um das Ziel, das diesem Konzept zugrunde liegt, zu erreichen, wurde eine sog. "Wissenstreppe" entwickelt.

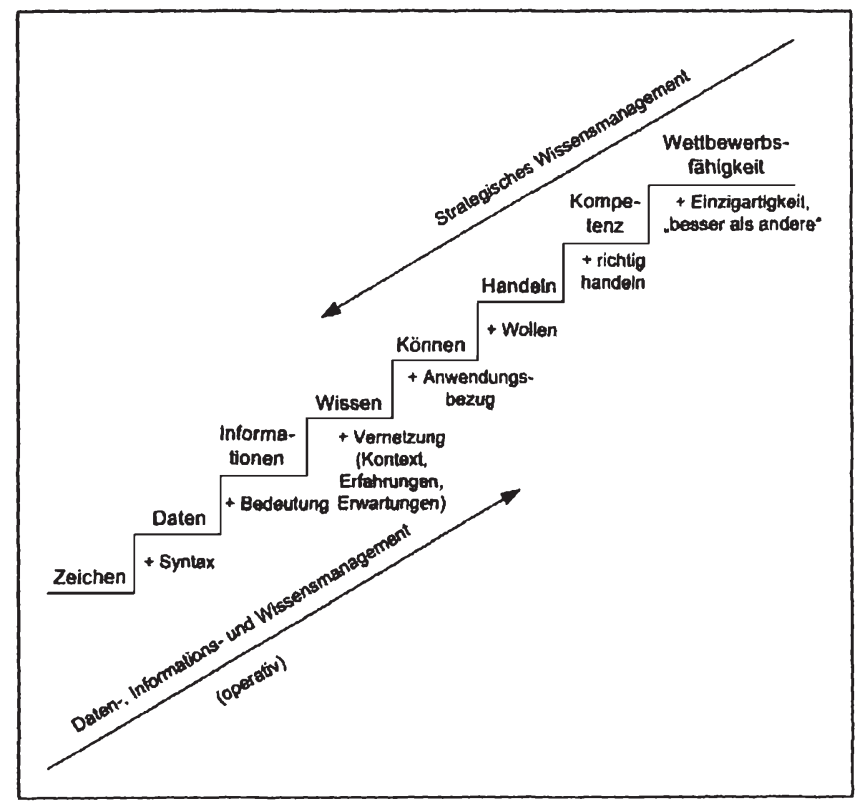

Abbildung 9: Wissenstreppe (North, 2002) 
Es kommt zu einer Eingrenzung von wissensmanagement-theoretischen Termini, die sich im Bereich Wissensmanagement als dominierend erweisen. Anlehnend an Albrecht (1993) wird "Wissen" als Ergebnis der Verarbeitung von Informationen durch das Bewusstsein definiert. Bezogen auf Probst et al. (1997) umfasst es die Gesamtheit der Kenntnisse und Fähigkeiten, die Personen zur Lösung von Problemen einsetzen. Somit ist Wissen ein Resultat eines individuellen Prozesses in einem spezifischen Kontext und manifestiert sich in Handlungen. Von "wissensorientierter Unternehmensführung" kann dann gesprochen werden, wenn alle Stufen der Wissenstreppe gestaltet werden (North, 2002).

Drei Handlungsfelder werden abgeleitet, die ein Wissensmanagement und eine wissensorientierte Unternehmensführung ausmachen. Einerseits gewährleistet ein strategisches Wissensmanagement, dass ausgehend von der obersten Führungsebene das Schaffen von Wettbewerbsvorteilen mittels des Faktors Wissen top-down vom gesamten Unternehmen getragen wird. Ferner vernetzt ein operatives Wissensmanagement sämtliche Informationen zu Wissen, Können und Handeln, wobei dem Transfer von individuellem Wissen zu kollektivem besondere Bedeutung zugesprochen wird. Das dritte Handlungsfeld wird durch ein Informations- und Datenmanagement gebildet, das das Fundament jedes Wissensmanagements darstellt. Denn als Voraussetzung für einen Wissensaufbau und transfer in einem Unternehmen zählen die Bereitstellung, Speicherung und Verteilung von Informationen (North, 2002).

Eine Wissensmarkt-Perspektive findet sich bereits bei Davenport/Prusak (1998) und hilft auch hier, das Management dieser knappen Ressource besser zu erfassen. Durch die auf den sog. "Wissensmärkten“ oftmals fehlende Transparenz, hervorgerufen durch eine Unvollkommenheit dieser Märkte, stehen Unternehmen gewissen Schwierigkeiten gegenüber, die dort angebotenen Wissensbestände zu vergleichen und trotz Informationsrückstandes eine für ihr Unternehmen optimale Entscheidung zu treffen. Besser als bereits kapitalisierte Ideen lassen sich potentielle Ideen und revolutionäre Zukunftsvisionen auf diesem Markt anbieten. Der Fokus dieses Konzepts wird auf organisatorische Rahmenbedingungen gelegt, 
die zu einem Gleichgewicht zwischen Wissensangebot und Wissensnachfrage führen sollen.

Somit führt dieses Konzept zu einer organisatorischen Neuausrichtung, indem eine Wissensorganisation im Unternehmen etabliert wird und sich dabei als komplementär zu einer hierarchischen/funktionalen Organisation, einer Prozessorganisation sowie zu einer Projektorganisation darstellt. Diese vierte Organisationsform ist als ein Gerüst zu sehen, in dem gemeinsame Kontexte geschaffen werden, in dem eine kompatible Problemlösungsfähigkeit gefördert wird und in dem Raum für Interaktionen von Menschen sowie eine physische und IT-Infrastruktur zur Repräsentation und Kommunikation von Wissen und übergreifenden Lernprozessen zur Verfügung steht (North, 2002).

Das diesem Ansatz zugrunde gelegte Wissenskonzept wird, indem auf zahlreiche Autoren rekurriert wird (Probst et al., 1997; Albrecht, 1993; Nonaka/Takeuchi, 1995; von Krogh/Roos, 1996; Sveiby, 1998), erörtert. North selbst trifft jedoch keine Entscheidung, auf welche Wissensdefinition er seinen Ansatz basiert. Der Fokus wird im Rahmen der Wissenstreppe auf den Transfer von individuellem Wissen zu kollektivem gelegt, der durch die Wissensspirale von Nonaka/ Takeuchi erfolgt. Die Mängel der Wissensspirale (im Kapitel "die Wissensspirale" bereits näher aufgeführt) beeinflussen auch die Stichhaltigkeit und Qualität dieses Ansatzes.

Dadurch, dass North den Fokus auf organisationale Rahmenbedingungen gelegt hat, lässt sich eine Orientierung dieses Ansatzes am Managementprozess erkennen, indem auch eigene Wissensmanagementprozesse entwickelt werden. Die entwickelte Wissenstreppe führt hin zu einer wissensorientierten Unternehmensführung, die sich durch die Fähigkeit, Wissen marktorientiert aufzubauen, abzusichern und optimal zur Generierung von Geschäftserfolgen zu nutzen, auszeichnet. Und obwohl North der wissensorientierten Unternehmensführung eine nähere Betrachtung schenkt, werden seine Ausführungen für die vorliegende Arbeit nicht übernommen. $\mathrm{Zu}$ oberflächlich geht North auf die verschiedensten Bereiche ein. So sollen wissensorientierte Unternehmen beispielsweise Lösun- 
gen für Kundenprobleme anbieten, die immer weniger arbeits- und kapitalintensiv und immer mehr wissensintensiv, schwer imitierbar bzw. derzeit oder mittelfristig nicht substituierbar sind (North, 2002). Doch auf welche Weise und durch welche Handlungen ein Unternehmen dies erreicht bzw. warum dies ein wissensorientiertes Unternehmen ausmacht, wird nicht erläutert.

Vor allem wird an seinem Ansatz und seinen Ausführungen die Breite der angeschnittenen Thematiken bemängelt, was unter Umständen zu der (bereits erwähnten) teilweisen oberflächlichen Behandlung einzelner Themenbereiche führt. So finden sich (teilweise mehr teilweise weniger) detaillierte Ausführungen zum Beispiel zu den Themen „Wissensgesellschaft”, „Wissen als Schlüsselressoure und Wettbewerbsfaktor", "ressourcenbasierter Ansatz", "Transaktionskostentheorie", "wissensorientierte Theorie der Unternehmensführung", "Wissensorganisation", "Wissensallianzen", "Communities of Practice", "organisationale Wissensbasis", "Wissen bewerten“, „Wissensbilanz", "Implementierung von Wissensmanagement" etc. 


\subsection{Das Schichtenmodell}

Die organisationale Wissensbasis steht im Mittelpunkt des Forschungsbemühens dieses Ansatzes. Eine Wissensbasis, jegliche Kenntnisse und Fähigkeiten umfassend, wird als Voraussetzung dafür gesehen, Handlungen und Problemlösungen in einem Unternehmen zu ermöglichen. Die organisatorische Wissensbasis repräsentiert den Wissensbestand, der für die Mitglieder einer Organisation im Prinzip verfügbar ist, und damit die Chance hat, in organisatorische Entscheidungen und Handlungen einzufließen (in Anlehnung an Kirsch, 1974).

Nur durch ein besseres Verständnis davon, was unter „im Prinzip verfügbares Wissen" gemeint wird, und aus welchen Komponenten sich eine kollektive Wissensbasis zusammensetzt, kann Wissen dem einzelnen Unternehmen erschlossen und zugänglich gemacht werden.

Anlehnend an die Forderung Bergers/Luckmanns (1999), dass unter Wissen alles verstanden werden solle, was in der Gesellschaft darunter gemeinhin subsumiert wird, kommt es in diesem Konzept zu der Berücksichtigung von Alltagswissen als einen integralen Bestandteil der Begriffsdefinition Wissen. Wissen als wissenschaftliche Erkenntnis wird um jene Kenntnisse, Fähigkeiten und Sinnstrukturen erweitert, die Handeln und soziale Koordination im täglichen Miteinander überhaupt erst möglich machen, ohne dass diese Form des Wissens bewusst vorher vorliegt und sprachlich formuliert wird. Diese Art von Wissen ist dem durch Polanyi (1962) geprägten „impliziten Wissen“ sehr ähnlich. Ein Schichtenmodell soll Aufschluss darüber geben, welches Wissen dem Unternehmen, in welchem Ausmaß, zur Verfügung steht (Pautzke, 1989). 


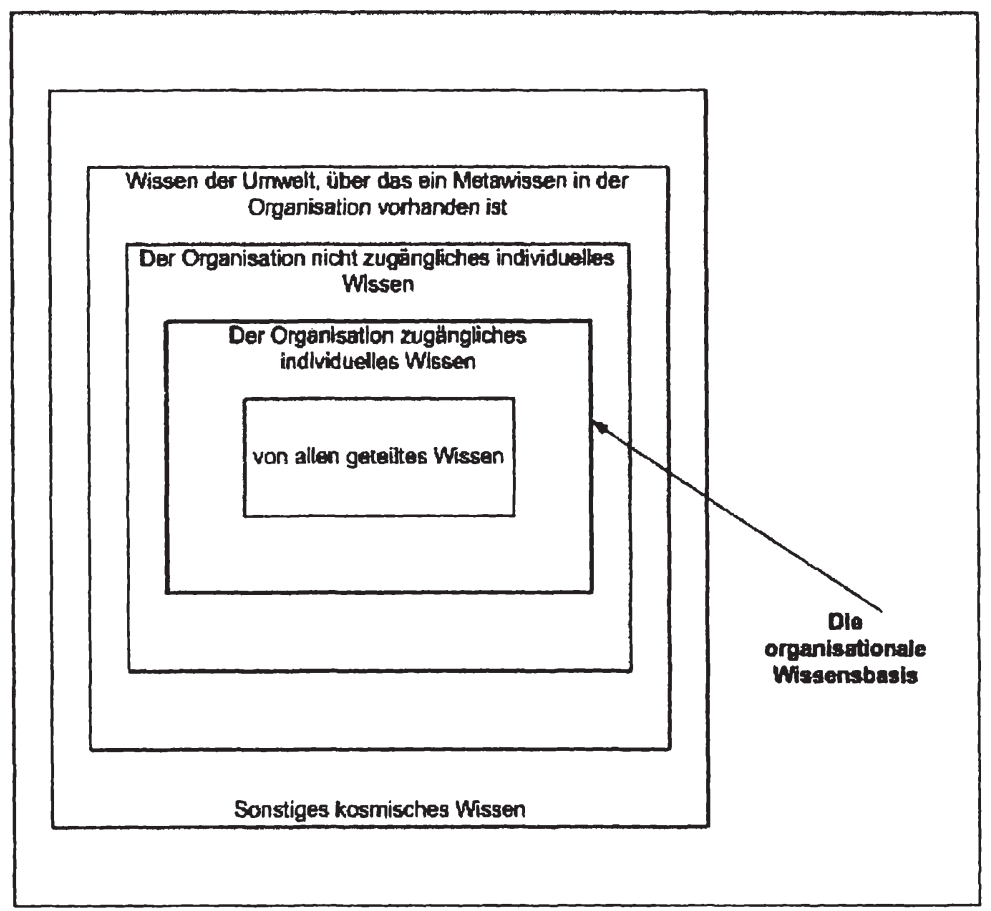

Abbildung 10: Das Schichtenmodell (Pautzke, 1989)

- von allen geteiltes Wissen

Dies wird als der Kern der organisationalen Wissensbasis gesehen. Es beinhaltet beispielsweise Daten über die Organisation (wie Name, Anschrift, Telefonnummer) und neben einem Begriffs-, Handlungs- und Rezeptwissen auch Grundsatzwissen (wie Daten über die Unternehmenskultur, unternehmensweite Welt- und Sinnbilder).

- der Organisation zugängliches und nicht zugängliches individuelles Wissen Wurden individuell zugängliche Wissensbestände artikuliert und dem Unternehmen zugänglich gemacht, kann das Unternehmen das normalerweise nur den Mitarbeitern zu eigene Wissen nutzen und somit Vorteile für das Unternehmen lukreieren. Es wird deutlich, dass sehr wohl Teile des individuellen Wissens dem 
Unternehmen nicht zugänglich sind, was darauf hinweist, dass eine organisationale Wissensbasis nicht mit der Summe des individuellen Wissens aller Unternehmensangehöriger gleichgesetzt werden kann.

- Wissen der Umwelt, über das ein Metawissen in der Organisation vorhanden ist Liegt Wissen im Wahrnehmungsbereich von Unternehmen, aber wurde es noch nicht explizit durch einen Lernprozess in den organisationalen Wissensbestand aufgenommen, so wird es dieser Kategorie zugeordnet. Das wahrgenommene Wissen von Kunden und Lieferanten im Unternehmensumfeld wird ferner mit eingeschlossen.

- Sonstiges kosmisches Wissen

Wissen, das keiner dieser Schichten zugeordnet werden kann und weder dem Individuum noch einem Unternehmen zugänglich ist, wird unter dem Begriff ${ }_{n}$ sonstiges kosmisches Wissen“ subsumiert. Nach Pautzke (1989) stellt dieser Bereich des Wissens auch den größten Teil von Wissen überhaupt dar, das sich als blinder Fleck außerhalb des Wahrnehmungsbereichs präsentiert.

Dieses horizontale Schichtenmodell wird um eine vertikale Schichtung erweitert, die die Gründe für die unterschiedliche Zugänglichkeit von Wissen zu finden helfen soll. Wissen erfährt eine Unterteilung in empirisches Wissen (Objektwissen, Oberflächenstruktur, Faktenwissen, Beobachtungswissen), heuristisch-analytisches Wissen (Theorie der Apparate) und den Bereich der Weltbilder und Sinnmodelle (Paradigma, Tiefenstruktur, Theories-of-Action) (Pautzke, 1989).

Die Anpassung an Entwicklungsschübe in der jeweiligen Umwelt einerseits sowie andererseits die Inszenierung von diesen Schüben wird durch eine organisatorische Lernfähigkeit ermöglicht. Den Rahmen für organisatorische Lernprozesse bilden die in einem Unternehmen vorzufindenden Oberflächenstrukturen (Hierarchie, Spezialisierung, Zentralisierung) als auch Tiefenstrukturen (Organisationskultur, Wissen der derivativen Lebenswelt). Lernen vollzieht sich als sequentielle Abfolge von Sinnmodellen. Stellvertretend für die gesamte Organisation verfol- 
gen sog. "Subkulturen“ (Pautzke, 1998, S. 228) die Entstehung, Erprobung und Konservierung neuen Wissens. Daraus kann ein verändertes Paradigma (eine neue Tiefenstruktur) entstehen und sogleich erprobt werden. Abhängig von den Machtkonstellationen innerhalb des Unternehmens und von einer Konsensfindung löst dieses neue Paradigma das alte ab (vgl. Kuhn, 1976).

Organisationen werden in diesem Ansatz als evolutionsfähige Gebilde betrachtet und die zentrale Rolle der Lernfähigkeit wird für die Evolution von Organisationen betont. Dabei richtet sich Pautzke gegen eine rationalistische Überbetonung von "Steuerbarkeit" und "Machbarkeit" und vertritt stattdessen einen "gemäßigten Voluntarismus". Es werden in diesem Ansatz die Lernfähigkeit und die Evolution von Organisationen als zentrale Themengebiete gewählt und dieser Ansatz behandelt die Bausteine einer Theorie des organisatorischen Lernens, die folglich in eine Organisationstheorie evolutionsfähiger Systeme resultieren. Diese Schwerpunktsetzung wird für die vorliegende Arbeit nicht übernommen und auch sonst stellen sich die Ausführungen von Pautzke für die Beantwortung der in der vorliegenden Arbeit aufgeführten Problemfelder als nicht unmittelbar dienlich dar. In diesem Ansatz wird Evolution als die Rationalisierung von Wissensbeständen und -strukturen verstanden und um einen anschlussfähigen Lernbegriff zu erarbeiten, ist die Auseinandersetzung mit der organisatorischen Wissensbasis vonnöten (Pautzke, 1989).

Weit verbreitet findet sich die "organisatorische Wissensbasis" als Schlagwort in der gängigen Literatur des Wissensmanagements. Die Begriffsbestimmungen ("Wissen“, "Organisation") begünstigen eine weitreichende Integration bekannter Ansätze. Diese Offenheit und Unschärfe der Begriffsdefinitionen (wie beispielsweise von "Wissen" und "Organisation") wirken sich jedoch negativ auf eine strukturierende und richtungweisende Funktion des Ansatzes aus. Somit kommt es selten zu einer Übernahme, geschweige denn Weiterentwicklung des Modells. Als Hauptkritikpunkt wird die unklare Bestimmung des Paradigmenwandels gesehen, da Pautzke (1989) keine klare Entscheidung trifft zwischen einem evolutionären und revolutionären Paradigmenwechsel. 
Das Individuum wird als Träger (Speicherort) jedes organisatorischen Wissens betrachtet und die Entwicklung eines originären Wissensbegriffs wird für eine Organisation unmöglich. Gleichzeitig wird die Rolle der Individuen nie explizit deutlich gemacht. Die mangelnde Verknüpfung der integrierten Einzeltheorien hat eine Unübersichtlichkeit zur Folge, wodurch keine Anknüpfungspunkte für ein Wissensmanagement oder Steuerungsprozesse organisatorischen Lernens zu finden sind (vgl. beispielsweise auch Wiegand, 1996). 


\subsection{Lebenszyklusmodell des Wissensmanagements}

Angelehnt an die Konzepte von Pautzke (1989) und Probst et al. (1997) wird auch das Wissensmanagement-Konzept von Rehäuser/Krcmar (1996) in Form parallel ablaufender Prozesse skizziert. Auf der Annahme basierend, dass Wissen den vierten Produktionsfaktor darstellt, ist es Aufgabe eines Wissensmanagements, neue Managementtechniken zur Planung, Steuerung, Organisation und Kontrolle zu entwickeln, die ermöglichen, dass Wissen separat in die Steuerungsund Berichtswege des Unternehmens aufgenommen wird. Ein Wettbewerbsvorteil kann erzielt werden und eine Ungleichverteilung von Information und Wissen in der Wirtschaft fördert dies. Als integraler Bestandteil des gesamten Unternehmensmanagements kommt dem Wissensmanagement große Bedeutung $\mathrm{zu}$, da es eine Querschnittsfunktion darstellt und untrennbar mit der Führungsaufgabe verbunden ist (Rehäuser/Krcmar, 1996).

Um infrastrukturelle und organisatorische Voraussetzungen für eine lernende Organisation zu schaffen, wird ein Wissensmanagement-System in einem Unternehmen etabliert, wobei im Zuge dessen dem Einsatz von Informations- und Kommunikationstechnologien für die Unterstützung eine bedeutende Rolle zugesprochen wird. Das Konzept der "lernenden Organisation" wird nicht näher erläutert, jedoch als eine für ein Unternehmen anzustrebende Form vorausgesetzt.

Auf drei Ebenen des Wissensmanagements entwickelt von Wollnik (1988) und Rehäuser (1991) (in Rehäuser/Krcmar, 1996) wird bei der Entwicklung und Argumentation dieses Konzeptes rekurriert: 


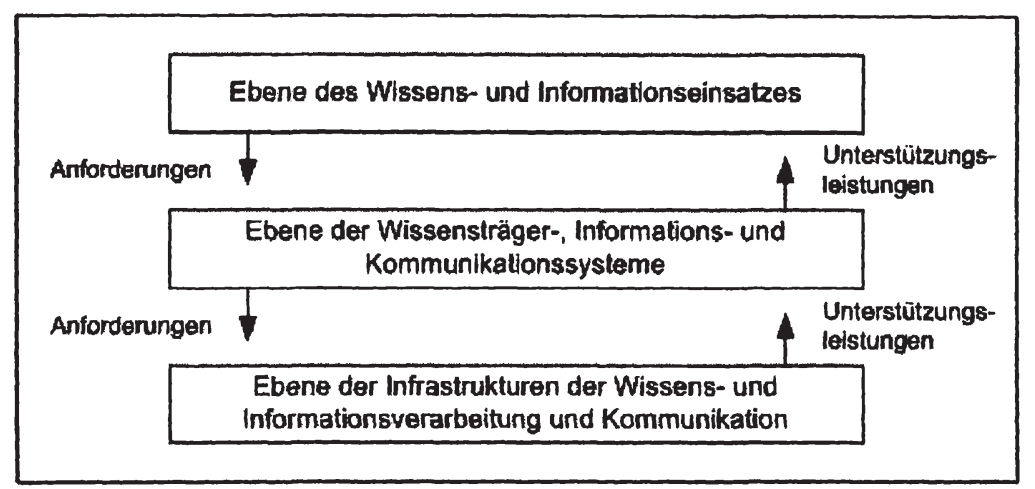

Abbildung 11: Die drei Ebenen des Wissensmanagements (Rehäuser/Krcmar, 1996)

Beginnend mit der Ebene des Wissens- und Informationseinsatzes werden der für ein Unternehmen festzustellende Wissensbedarf und seine Deckung durch das Wissensangebot geplant, organisiert und kontrolliert. Systeme personeller, organisatorischer und technischer Natur, die zur Deckung des Wissensbedarfs in einem Unternehmen etabliert worden sind, stellen dabei als Wissensträger-, Informations- und Kommunikationssysteme Hilfestellungen zur Verfügung, die vor allem eine Strukturierung und Gestaltung der Wissensträger- und Informationsressourcen sowie der Wissens- und Informationsquellen beinhalten. Die unterste Ebene, die wiederum Unterstützungsleistungen bietet, gewährleistet die Bereitstellung der Wissensträger- und Informationsressourcen sowie der Wissens- und Informationsquellen und der dafür notwendigen Technologien.

Aufbauend auf diesen drei Ebenen und auf dem Schichtenmodell der organisatorischen Wissensbasis (Pautzke, 1989), lassen sich Aufgaben des Wissensmanagements ableiten und in Managementphasen aufspalten, was in Form des Lebenszyklusmodells des Wissensmanagements illustriert wird (Rehäuser/Krcmar, 1996): 


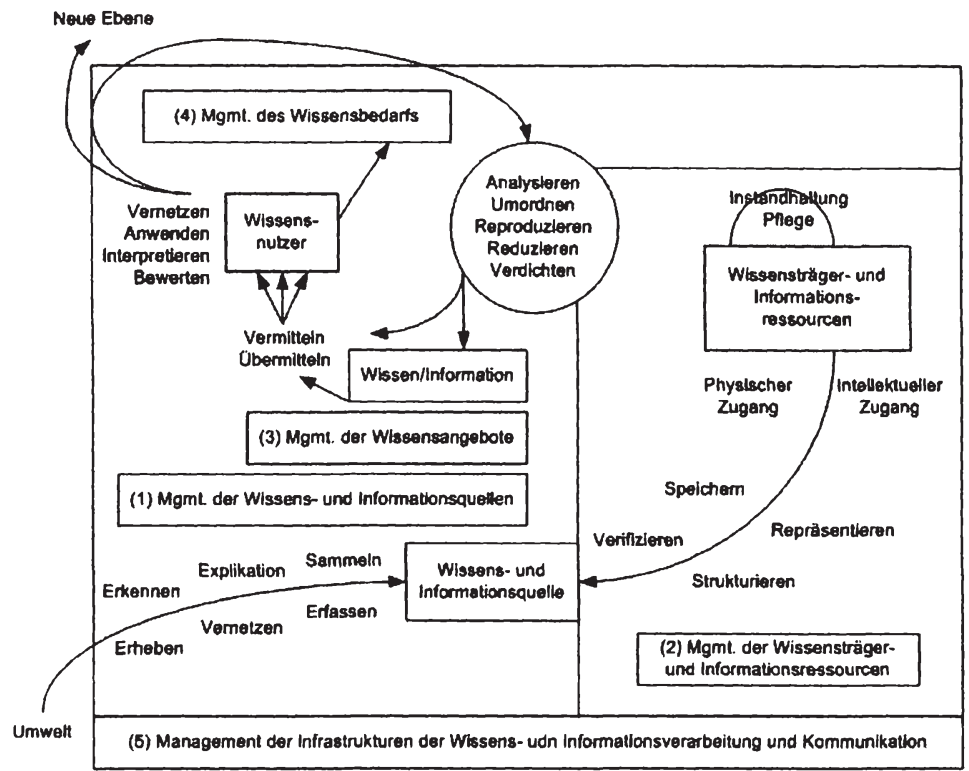

Abbildung 12: Lebenszyklusmodell (Rehäuser/Krcmar, 1996)

- Management der Wissens- und Informationsquellen

Um mit Wissen arbeiten zu können, muss man es vorweg im Unternehmen ausfindig machen. Es inkludiert eine fortwährende Suche nach neuen Wissensbeständen, die die bereits bestehenden Wissensressourcen erneuern und/oder erweitern.

- Management der Wissensträger- und Informationsressourcen

Einem guten Management obliegt die Aufgabe, das dem Unternehmen noch nicht über Wissensträger allgemein zugängliche Wissen in ein solches zu transferieren, wobei man sich dabei meist der technischen Applikationen des organisationalen Informationssystems bedient.

\section{- Management des Wissensangebots}

Das Wissensangebot eines Unternehmens wird fast gänzlich durch technische Wissens- und Informationsquellen getragen und diese müssen einer Strukturie- 
wendigen Wissensbestände leicht zu finden und zu verwerten. Hierbei ist auf wissenslogistische Prinzipien Acht zu nehmen.

- Management des Wissensbedarfs

Werden organisationale Wissensbestände zur Lösung von Frage- und Problemstellungen herangezogen, unterliegen diese fortwährend einer Veränderung und Interpretation durch die Personen, die auf Wissensressourcen rekurrieren. Somit kann die auf technischen Wissensträgern festgehaltene Information niemals als etwas Statisches und Absolutes angesehen werden, sondern unterliegt einer ständigen Veränderung und Erweiterung. Dies macht es notwendig, das bereitgestellte Wissensangebot von den einzelnen Benutzern nach ihrer Verwertbarkeit und Tauglichkeit bewerten und gegebenenfalls anregen zu lassen, sodass dieses erweitert wird, was in dieser Phase des Lebenszyklus dem Management obliegt.

- Management der Infrastrukturen der Wissens- und Informationsverarbeitung und Kommunikation

Um jedoch den Blickwinkel im Rahmen dieses Wissensmanagement-Konzepts nicht zu eng zu wählen, wird auch der personellen, organisatorischen sowie technologischen Infrastruktur eine Bedeutung zugesprochen. So sollen auf der Ebene der Personalpolitik die Mitarbeiter eines Unternehmens langfristig mittels entsprechender Anreizstrukturen an das Unternehmen gebunden werden sowie mittels einer im Unternehmen optimal integrierten Technologie vernetzt sein. Über organisationale Strukturen wird ein Wissensmanagement überhaupt erst ermöglicht.

Beginnend mit einem informationstheoretisch inspirierten Wissensverständnis wird Wissen von den Termini "Daten" und "Informationen" unterschieden. Dieser Ansatz bietet eine erste Ordnung, jedoch können auf entscheidende Fragen keine Antworten gefunden werden. Beispielsweise bleibt ungeklärt, welche Informationen schlussendlich zu Wissen werden. Diese Beliebigkeit lässt keine Auswahlregeln erkennen. Wissen entsteht in einem bestimmten Kontext und in einer bestimmten Anwendungssituation. Diese Idee die zweckorientierte Vernetzung von 
Informationen als Wissen zu bezeichnen, lässt jedoch eine konkrete Spezifizierung von Wissen offen. Es fehlt ein Verfahren, das anzeigen würde, unter welchen Umständen Informationen zu Wissen werden können ( $\mathrm{vgl}$. beispielsweise auch Schreyögg/Geiger, 2003). Somit wird dieses Wissensverständnis in dieser Form nicht für die vorliegende Arbeit übernommen.

Dieser Ansatz, der die Ausrichtung auf Unternehmensziele nicht erkennen lässt und auch kein Instrumentarium zur praktischen Umsetzung bietet, weist in seinen Ausführungen auch keine Handlungsorientierung auf. Es wird an einem technokratischen Managementprozess festgehalten. Das Modell selbst ist rein beschreibender Natur und weniger handlungsorientiert. Eine empirische Überprüfung dieses Konzepts ist bisher nicht erfolgt (vgl. auch North, 2002; Neumann, 2000). Wissensmanagement zeichnet sich in diesem Ansatz durch das Zusammenspiel der oben dargestellten Managementphasen aus; wie bereits im Rahmen der Darstellung des Ansatzes von Probst et al. (1997) festgehalten wurde, wird in dieser vorliegenden Arbeit jedoch unter Wissensmanagement nicht das Zusammenspiel von in einem Unternehmen extra definierten - meist wissensintensiven - Managementprozessen bzw. -phasen verstanden, sodass das Konzept von Rehäuser/Krcmar als Grundlage der vorliegenden Arbeit ebenfalls nicht in Frage kommt. 


\subsection{Abschließende Betrachtung}

In einer Zeit fortschreitender Entwicklungen am Informationssektor, einer unaufhaltsamen Globalisierung und verstärkten Tendenzen zu branchendominierenden Machtkonzentrationen durch Fusionen, Mergers \& Acquisitions bzw. netzwerkartigen Unternehmensverbünden intensivieren Unternehmen die Suche nach jenen Faktoren, die im Verborgenen der Organisation, in der sog. Black Box liegen, die Unsicherheiten zu reduzieren im Stande sind, die etwas Bestehendes, die rasanten Entwicklungen Überdauerndes, besitzen und Kontinuität gewährleisten, wie z.B. Lernen, Kreativität und letztendlich Wissen. Wissen hat gleichzeitig jedoch auch etwas Verhinderndes und Unbewegliches an sich, denn es wird daran festgehalten und steuert selektiv die Aufnahme neuen Wissens nach dem Kriterium der Aufrechterhaltung und Kontinuität (vgl. Neumann, 2000).

Das Spannungsfeld zwischen Bewahrung und Veränderung vor dem Hintergrund einer notwendigen Entfaltung der Produktivkraft von Wissen stellt die Theorie und Praxis vor neue Herausforderungen. Die zuvor präsentierten, ausgewählten Wissensmanagement-Ansätze spiegeln die diskursive, intensive und recht vielfältige Auseinandersetzung mit Wissen in und von Organisationen wider. Eine zu enge Darstellung von Wissensmanagement kann dazu führen, dass wissensmanagementtheoretisch relevante Aspekte in der Untersuchung von Organisationen ausgeblendet werden und ein umfassendes Verständnis von Wissensmanagement verhindert wird. Die theoretische Ausrichtung eines WissensmanagementKonzepts beeinflusst wesentlich, was unter Wissen subsumiert, wie Wissensmanagement gestaltet und worauf der Schwerpunkt des Konzepts gelegt wird.

Aus diesem Grund stand im Mittelpunkt dieses Kapitels, die unterschiedlichen Ideen zum Bereich Wissensmanagement darzustellen, um daraus jene Ideen für die Entwicklung des theoretischen Bezugsrahmens zu nutzen, die für die Beantwortung der dieser Arbeit zugrunde liegenden Forschungsfragen geeignet sind. Unterschiedliche Forschungsfragen erfordern unterschiedliche theoretische $\mathrm{He}$ - 
rangehensweisen an den Bereich Wissensmanagement, wodurch keiner Forschungsrichtung grundsätzlich ein Vorrang eingeräumt werden soll.

Aus der Aufarbeitung der gängigsten Wissensmanagement-Ansätze im betriebswirtschaftlichen Bereich lassen sich erste Schlüsse über die Verwendungsmöglichkeiten von Wissensmanagement für die Beantwortung der Forschungsfragen der vorliegenden Arbeit ziehen. Diese Arbeit ist in einem funktionalistisch geprägten Paradigma verortet. Somit wird von den dargestellten Ansätzen die grundlegende Herangehensweise an Wissensmanagement als Instrument der Unternehmensführung übernommen. In welcher Form ein Wissensmanagement gestaltet werden soll, hängt stark von der Betrachtungsweise von Wissen selbst ab. „Wissen" ist auf alle Fälle positiv konnotiert, da es gut und wichtig ist es zu besitzen und da ein konstruktiver Umgang mit dieser Ressource für Mensch und Organisation von erheblichem Vorteil ist. Es wird in dieser Arbeit auf die Begriffsdefinition von Polanyi (1962) rekurriert.

Die aktuelle Modeströmung des Wissensmanagement - wie bspw. anhand der dargestellten gängigsten Wissensmanagement-Ansätze in den vorangegangenen Kapiteln gezeigt werden konnte - konzentriert sich primär auf das Aufspüren von Wissen in Organisationen. Meist wird dieses in den Köpfen der jeweiligen Organisationsmitglieder vermutet und dort lokalisiert, mit dem Ziel, es explizit zu machen und vom Einzelnen zu entkoppeln, um es den Organisationen verfügbar zu machen. Die Quelle von Wissen wird in der vorliegenden Arbeit ebenfalls in den Köpfen von Individuen ausgemacht. Eine gleichbedeutende Aufmerksamkeit verdient in dieser Arbeit jedoch auch das implizite Wissen und das Wechselspiel zwischen explizitem und implizitem Wissen soll näher erörtert werden.

Auf organisationaler Ebene gestaltet sich die Suche nach dem überindividuellen und vom einzelnen Mitarbeiter eines Unternehmens unabhängigen Wissen um einiges schwieriger. Nicht nur in den Köpfen, sondern auch in den personenunabhängigen anonymisierten Regelsystemen, in Standardverfahren, Leitlinien, Kodifizierungen, Arbeitsprozessbeschreibungen usw. ist Wissen eingebettet. 
Darin wird der in dieser Arbeit zu elaborierende Wissensmanagement-Ansatz begründet. Es gilt daher eine Organisationstheorie zugrunde zu legen, die hierfür ein Fundament bieten kann.

Die Theorie der Neuen Institutionenökonomie wurde ausgewählt, ein solches theoretisches Fundament zu bilden. Auf Basis der Grundannahmen dieser Theorie wird in den folgenden Kapiteln eine mögliche Herangehensweise an Wissensmanagement entwickelt. Im Mittelpunkt sollen Institutionen als Spielregeln von Organisationen stehen. Aufgrund der individualistischen Positionierung dieser Theorie wird dem Individuum eine besondere Aufmerksamkeit geschenkt. An dieser Stelle wird auch der oben bereits erwähnte Schwerpunkt auf das Wechselspiel zwischen explizitem und implizitem individuellen Wissen gelegt. Es soll ein Bezugsrahmen für ein Wissensmanagement entwickelt werden, der einerseits den internen organisationalen Kontext und seine Dynamiken erfasst und andererseits den Einfluss von gesellschaftlichen und institutionellen Rahmenbedingungen berücksichtigt. Somit soll eine theoretische Erweiterung der gängigsten Wissensmanagement-Ansätze um die Theorie der Neuen Institutionenökonomie als Organisationstheorie erfolgen. 
III Wissensmanagement in Anlehnung an die Neue Institutionenökonomie

5. Die Neue Institutionenökonomie

Die Theorie bestimmt, was wir beobachten können.

Albert Einstein

\section{1. Überblick}

Die Neue Institutionenökonomie und die darin inkludierten Strömungen werden in den vertragsbasierten Ansätzen zusammengefasst. Sie erhielten Anfang der Siebziger einen Aufschwung und werden mit den Werken von beispielsweise Williamson (1975; 1989), North (1981; 1990), Alchian/Demsetz (1973), Arrow (1974) assoziiert, basierend auf den Werken von beispielsweise Coase (1937), Commons (1934) oder Knight (1922). Es kommt zu einem Neuaufleben des Erkenntnisinteresses von institutionenökonomischen Unternehmenstheorien, wobei im Rahmen dieser Theoriestränge Unternehmen, Haushalte und Märkte seit jeher zentrale Untersuchungsgegenstände darstellen. Diese Schwerpunktsetzung geht auf die Neoklassik (Marshall, 1920; Jevons, 1970; Menger, 1840-1921; Walras, 1834-1910; Mises, 1881-1973; Hayek, 1899-1992; auch oftmals Marx, 18181883) zurück, wobei im Rahmen der Unternehmens- und Haushaltstheorien die Gewinn- und Nutzenfunktion maximiert wurden, um Aussagen über das Angebot und die Nachfrage an Arbeit und produzierten Gütern zu erhalten. Ein bedeutender Beitrag der neoklassischen Lehre erschließt sich in der Erkenntnis, dass die Anwendung des freien Preismechanismus unter den Bedingungen der vollkommenen Konkurrenz zu einer optimalen Allokation der Produktionsfaktoren führt, was der Notwendigkeit der Existenz von Unternehmen jedoch jeglichen Boden entzieht. Die unternehmensinternen Vorgänge wurden nicht gestaltet, da sie als 
„Black Box“ betrachtet und lediglich durch zugrunde liegende Produktions- und Kostenfunktionen charakterisiert wurden.

Obgleich die neoklassischen Erkenntnisse für das Verständnis von Marktvorgängen bedeutend waren, so war ihnen fortwährend ein großes Kritikpotential inhärent. Die mangelnde Realitätsnähe und die Abstraktheit der Ausführungen kritisierend bildete sich bereits Anfang der zwanziger und dreißiger Jahre ein Forschungsbereich, der sich zum Ziel setzte, eine an realen Erscheinungen interessierte Unternehmenstheorie zu gestalten, wobei hier vier Strömungen unterschieden werden ( $\mathrm{vgl}$. Meuthen, 1997): erstens die neue politische Ökonomie, die zu erklären versucht, wie eine Gesellschaft Regeln aufstellt, mit der sie letztendlich entscheidet, unter welchen Bedingungen das zu errichtende System operieren wird; die ökonomische Analyse des Rechts, wobei eine ökonomische Effizienzanalyse auf Fragen der Rechtsprechung durchgeführt wird. Dann folgen drittens die Evolutorik, die basierend auf dem Prinzip der unsichtbaren Hand (in der Tradition von Smith, Menger) die Entstehung und Entwicklung wirtschaftlicher und anderer sozialer Institutionen betrachtet, und zuletzt die Neue Institutionenökonomie, in deren Mittelpunkt die Analyse der Entstehung und Veränderung wirtschaftlicher Institutionen steht.

Dass innerhalb letzterer Theorie nochmals zwei Strömungen (die ältere und neuere Prägung) aufgetreten sind, soll der Vollständigkeit halber erwähnt werden. So lassen sich zu der älteren Prägung der amerikanische Institutionalismus (Commons, Mitchell) und die deutsche historische Schule (Roscher, Hildebrand, Schmoller) hinzuzählen. Beide konzentrierten sich vor allem auf die Kritik an der Neoklassik. Die letztere Strömung, die unter dem Namen die "Neue Institutionenökonomie" zusammengefasst wird (dieser Begriff stammt von Williamson, 1975), wird von denjenigen Autoren gebildet, die eingangs erwähnt wurden; sie wird den weiteren Ausführungen dieser Arbeit zugrunde gelegt. Institutionen sind für den Wirtschaftsprozess von grundlegender Bedeutung und die institutionelle Ordnung übt großen Einfluss auf das Verhalten ökonomischer Akteure aus (Richter/Furubotn, 1996). Im Zuge der Neuen Institutionenökonomie werden Institutio- 
nen als abhängig von der jeweiligen Transaktion, den Kosten und der Effizienz gesehen, sodass sie mit diesen drei Faktoren erklärt werden (vgl. Williamson, 1975; 1985; North, 1981).

Die Neue Institutionenökonomik stellt kein einheitliches Theoriengebäude dar und besteht vielmehr aus mehreren methodologisch verwandten Ansätzen (vgl. Richter, 1992; Furubotn/Richter, 2000). Ökonomische Theorien zeichnen sich prinzipiell durch ihre axiomatische Basis aus, die durch die Annahmen des methodologischen Individualismus und der individuellen Nutzenmaximierung definiert ist. Den Scheidepunkt ökonomischer Theorien bildet die Rationalitätsfrage: einerseits kann die Annahme getroffen werden, dass Akteure vollkommen rational handeln, was die neoklassischen Theorien unterstellen und somit "Rationalität" nicht weiter thematisieren. Andererseits bildet die Annahme der "begrenzten Rationalität" den Ausgangspunkt für die Fragestellungen der institutionenökonomischen Theorien (vgl. Picot/Diet//Franck, 1999; Kappelhoff, 1997).

Im Rahmen des methodologischen Individualismus wird der Fokus auf das Individuum gelegt. Institutionen (Organisationsstrukturen) werden als das Ergebnis der Handlungen und Entscheidungen individueller Akteure verstanden (vgl. Picot/ Diet//Franck, 1997).

Basierend auf dieser Annahme wird das menschliche Verhalten ebenfalls aus der Sicht des Individuums betrachtet und über Zielsetzungen definiert, die sich als individuelle Nutzenfunktionen beschreiben lassen. Individuelle Akteure verfolgen klar definierte Ziele und streben danach ihren Nutzen zu maximieren, wobei der individuelle Nutzen aus einer Vielzahl an Faktoren bestehen kann. Es muss konsequenterweise davon ausgegangen werden, dass einzelne Akteure unter Umständen ihre Ziele auch auf Kosten und zum Schaden anderer Akteure sowie unter Verletzung geltender Regel verfolgen. Solche Handlungsweisen, bei denen ein Akteur zur Durchsetzung eigener Interessen die mögliche Schädigung anderer bewusst in Kauf nimmt, werden unter den Begriffen ${ }_{n}$ opportunistisches Verhalten“ bzw. „Opportunismus" zusammengefasst (self-seeking, with_-gyile" „wie es 
Williamson, 1975 auch beschreibt). Der Unterschied zur individuellen Nutzenmaximierung wird insofern gezogen, dass hierbei wirtschaftliches Handeln aus der Perspektive des einzelnen Akteurs betrachtet wird, wohingegen Opportunismus die Handlungskonsequenzen des in dieser Weise nutzenmaximierenden Akteurs aus der Sicht eines externen Beobachters reflektiert. Mit der Verhaltensannahme des Opportunismus wird nicht davon ausgegangen, dass Individuen automatisch ein solches Verhalten an den Tag legen, jedoch wird bei der Gestaltung von Austauschverhältnissen die prinzipielle Möglichkeit berücksichtigt, dass Akteure z.B. Informationsassymetrien oder einseitige Abhängigkeitsverhältnisse in schädigender Weise zu ihren Gunsten aus-nutzen (vgl. Picot/Diet//Franck, 1997).

Diese beiden Kernannahmen (methodologischer Individualismus sowie individuelle Nutzenmaximierung) können in weiterer Folge Gegenstand unterschiedlicher Präzisierung und Auslegung sein. So scheiden sich ökonomische Theorien hinsichtlich der Frage, ob Akteure vollständig und zutreffend über die Welt informiert sind (objektive Rationalität) oder lediglich unvollständig bzw. fehlerhaft (begrenzte Rationalität). Erstere gehen davon aus, dass das Wissen und die Informationsverarbeitungsmöglichkeiten des Akteurs unbegrenzt sind. Welt und Weltsicht des Akteurs sind dann per definitionem ein und dasselbe. Somit trifft ein Individuum stets die Entscheidung, die im Hinblick auf seine Bedürfnisse objektiv (in der "wahren" Welt) die beste ist, da es automatisch zutreffende und vollständige Informationen über die reale Welt hat. Dieses Verhalten wird als "vollständig rational" bezeichnet. Somit gilt: "[a] completely rational individual has the ability to foresee everything that might happen and to evaluate and optimally choose among available courses of action, all in blink of an eye and at no cost' (Kreps, 1990b, S. 745, in: Furubotn/Richter, 2000).

Hingegen gehen Theorien der zweiten Ausprägung davon aus, dass aufgrund des begrenzten Wissensstandes und der begrenzten Informationsverarbeitungsmöglichkeiten Individuen eine subjektive Wahrnehmung der Welt haben und ihr Verhalten nicht als objektiv rationales beschreibbar ist. Vielmehr wählt ein Indivi- 
duum aus einer Menge an Handlungsalternativen diejenige aus, die gemäß seiner als konsistent vorausgesetzten Präferenzordnung optimal ist (vgl. Picot/Diet//Franck, 1997). Jedes Handeln ist somit in der vorhandenen Situation abgestimmt auf das individuelle Präferenzmuster rational. Das Menschenbild, das diesem Rationalitätsprinzip zugrunde liegt, zeigt einen Akteur, der aktivistisch ist, d.h. zielgerichtet auf seine Umwelt einzuwirken versucht (vgl. Kappelhoff, 1997). Das Verhalten wird als "begrenzt rational" bezeichnet (vgl. Simon, 1957, 1991; Williamson, 1975). Ein Akteur ist sich rational dem Zweck, der Mittel und der Folgen bewusst und wägt Ziele, Mittel und Folgen gegeneinander ab. Das Abwägen in Entscheidungssituationen kommt einer Umsetzung von Handlungsrestriktionen und individuellen Präferenzen in absichtsvolles Handeln gleich. „Absichtsvolles Handeln" zeigt, dass Individuen ihrem Verhalten eine rationale Absicht zugrunde legen, die sie jedoch aufgrund ihres begrenzten Wissens nur begrenzt einzulösen imstande sind. Der Handlungsspielraum von rationalem Handeln wird oftmals durch Restriktionen beschränkt, die bspw. die physische Umgebung, das wirtschaftliche Umfeld, Preise, das finanzielle Budget, die verfügbare Zeit, angedrohte oder verhängte Sanktionen umfassen. Ferner wird das Verhalten durch die subjektiven Haltungen, Einstellungen, Wünsche und Präferenzen eines jeden Akteurs bestimmt. Diese Präferenzen werden anhand des Nutzens jeder Handlungsalternative in Rangordnungen, den sog. Präferenzordnungen, strukturiert (Petermann, 2001).

Die Entscheidung für eine dieser Konzeptionen des ökonomischen Akteurs bildet den Scheidepunkt, an dem sich die Ökonomik in Teilparadigmen trennt (vgl. Picot/Diet//Franck, 1997).

In den Institutionenökonomischen Ansätzen wird in der einen oder anderen Form davon ausgegangen, dass die begrenzte Rationalität von Akteuren Schäden verursachen kann, die sich bspw. als entgangener Produktivitätszuwachs durch falsche Arbeitsteilung sowie Spezialisierung und/oder als überhöhte Tausch- bzw. Abstimmungskosten manifestieren können. Durch Koordination und Motivation 
gilt es mithilfe von Institutionen, sog. Rationalitätsinstrumenten, diese Schäden zu minimieren und somit ein hohes Maß an Bedürfnisbefriedigung zu erreichen.

Geht man von völlig rationalen Akteuren aus, wie es neoklassische und industrieökonomische Ansätze tun, so entzieht man Rationalitätsinstrumenten die Legitimationsbasis, da diese von Akteuren, die unbegrenzt über Rationalität verfügen, nicht gebraucht werden. Auch wenn feststeht, dass Individuen nicht vollständig informiert sind und daher nicht objektiv rational sein können, so ist dies kein Grund zur Ablehnung einer Ökonomik mit rationalen Akteuren. Institutionen sind in diesen Ansätzen Instrumente ökonomischer Akteure zum Aufbau und zur Verteidigung von bzw. gegen Marktmacht.

Das letzte Teilparadigma wird von den evolutorischen Ansätzen gebildet, die sich wie die institutionenökonomischen Ansätze auf die Verhaltensannahme der begrenzten Rationalität stützen. Sie radikalisieren diese Annahme jedoch noch weiter. Rationalitätslücken werden in institutionenökonomischen Ansätzen durch rationale Institutionengestaltung ausgeglichen, jedoch der Erfolg eines solchen Unterfangens wird in evolutorischen Ansätzen angezweifelt. Institutionen können nicht gänzlich Ergebnis von menschlicher Planung sein, sondern werden auch durch eine gewisse Pfadabhängigkeit von Evolutionsprozessen geprägt.

In der vorliegenden Arbeit werden Annahmen der institutionenökonomischen Ansätze zugrunde gelegt. Sie rekurrieren in ihren Ausführungen auf sog. „Rational-Choice-Modelle". Diese Modelle, die auch in der Rational-Choice-Theorie zusammengefasst werden, stellen eine allgemeine, präzise ausformulierte Sozialtheorie dar. Der sozialphilosophische Hintergrund dieser Theorie geht auf die These zurück, dass das Individuum der Gesellschaft vorgeordnet sei und dass die Gesellschaft somit als Resultante zielorientierter Handlungen autonomer Akteure verstanden wird. Die zentralen Ideen sind fester Bestandteil des strukturellindividualistischen Ansatzes in den Sozialwissenschaften, der sich aus den Unzulänglichkeiten des neoklassischen Modells des "homo oeconomicus" einerseits 
und des soziologischen Modells des "homo sociologicus" andererseits entwickelt hat (vgl. Petermann, 2001).

Rational-Choice-Theorien werden benötigt, um das Verhalten der Akteure auf der Mikroebene zu erklären. Hierbei müssen drei Bedingungen erfüllt sein. $\mathrm{Zu}$ allererst stellt das Individuum den Ausgangspunkt der Theorien dar und diese $\mathrm{Be}$ dingung wird durch die Grundannahme des methodologischen Individualismus erfüllt, indem Systemverhalten auf der Makroebene durch Akteurshandeln auf der Mikroebene erklärt wird. Ferner sehen sich Akteure mindestens zwei Handlungsalternativen gegenüber. Diese Bedingung ist notwendig, damit überhaupt eine Entscheidungssituation vorliegt. Eine Alternative kann immer eine solche sein, eine Handlung nicht auszuführen. Zum dritten enthält jede Rational-ChoiceTheorie eine Entscheidungsregel, die angibt, welche Handlung ein Akteur ausführen wird (vgl. Petermann, 2001).

Mit Hilfe des Makro-Mikro-Schemas (siehe Abbildung) kann das Zusammenspiel von gesellschaftlicher Makroebene und individueller Mikroebene analysiert werden. Die Randbedingungen werden durch den Makro-Mikro-Übergang spezifiziert, der handlungsleitend auf die Mikroebene einwirken. Ausgehend von einer Verankerung des rationalen Handelns in der Mikroebene folgt die Handlung selbst einem ahistorisch verstandenen abstrakten Optimierungskalkül. In einem weiteren Transformationsprozess ergibt sich dann das Handlungsresultat auf der Makroebene (Kappelhoff, 1997). 


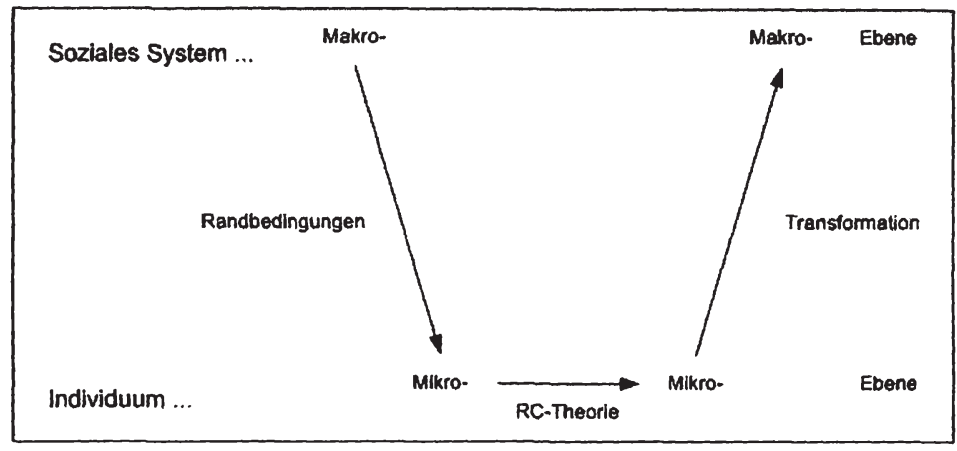

Abbildung 13: Makro-Mikro-Makro-Schema (Kappelhoff, 1997, S. 221)

Die Neue Institutionenökonomie ist in einem funktionalistischen Paradigma verortet und durch eine objektivistische Orientierung gekennzeichnet. Dieser realistisch-individualistische Ansatz betrachtet Institutionen als Regelmäßigkeiten repetitiver Interaktionen, als Gewohnheiten, Governance Strukturen bzw. soziale Vereinbarungen, die für die Akteure einerseits eine Anreizstruktur und andererseits Beschränkungen darstellen (Williamson, 1985; North 1981). Diese Regeln schaffen einen Handlungsspielraum, in dem sich die Akteure teilweise aus reinem Selbstinteresse, aber auch aus Gehorsam bewegen, um negative Sanktionen zu vermeiden.

Soziale Strukturen werden als Manifestationen sozialer Rollenerwartungen gesehen und in Form von Governance Strukturen der Märkte, Hierarchien und Hybriden (vgl. beispielsweise Williamson, 1975; 1985) bzw. der Steuerungs- und Kontrollsystemen (vgl. beispielsweise Alchian/Demsetz, 1972) näher untersucht. Die Neue Institutionenökonomie positioniert sich basierend auf einem regulativen Träger, im Zuge dessen die handlungsbegrenzenden und -regulierenden Aspekte von Institutionen von Interesse sind. Normative Werte werden, konträr zu dem soziologisch geprägten Neoinstitutionalismus, als extern gegeben betrachtet.

Durch die dominierende Stellung der Neuen Institutionenökonomie innerhalb der Neuen Institutionentheorien bietet sie sich als theoretisches Fundament eines

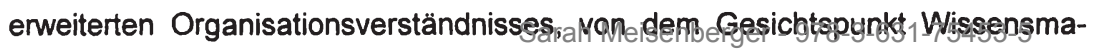


Durch die dominierende Stellung der Neuen Institutionenökonomie innerhalb der Neuen Institutionentheorien bietet sie sich als theoretisches Fundament eines enweiterten Organisationsverständnisses, von dem Gesichtspunkt Wissensmanagement aus, an. Aus dem umfassenden Erklärungsrahmen kann geschöpft werden, um Strukturen, Verhaltenswirkungen und die Effizienz von Institutionen im Rahmen eines Wissensmanagements zu durchleuchten. Organisationen werden in weiterer Folge vor allem als Organisationsstrukturen betrachtet. In der Terminologie der Institutionenforschung ist die Organisationsstruktur ein System von Institutionen. Sie soll den Handlungsspielraum der Unternehmensmitglieder eingrenzen und ihr Verhalten zielgerichtet steuern, um auf diese Weise Koordination und Motivation zu gewährleisten (Picot/Diet//Franck, 1997, S. 31). 


\subsection{Institutionen - die Spielregeln von Organisationen}

Im Mittelpunkt der Neuen Institutionenökonomie steht die Analyse von Institutionen, in deren Rahmen der ökonomische Austausch vollzogen wird. Das Ziel der Institutionenanalyse besteht darin, die Struktur, die Verhaltenswirkungen, die Effizienz und den Wandel von ökonomischen Institutionen zu erklären (Ebers/ Gotsch, 1999). Die Grundelemente des Wirtschaftens von Unternehmen werden von Arbeitsteilung, Spezialisierung, Tausch und Abstimmung geprägt. Strukturen der Arbeitsbeteiligung, der Spezialisierung, des Tausches sowie der Abstimmung sind hierbei nicht das Ergebnis eines naturgesetzlich vorausbestimmten Prozesses, sondern geplante und ungeplante Folgen menschlicher Entscheidungen und Handlungen. Mängel im Sinne von ungünstigen Gestaltungen können in allen vier Bereichen auftreten. Die Gründe hierfür sind mannigfaltig. So können beispielsweise Situationen, in denen Aufgabenelemente, die keinerlei Gemeinsamkeiten haben und dennoch zum Aufgabenbereich einer Person bzw. einer Organisationseinheit zusammengefasst werden, zu einer unproduktiven Arbeitsteilung bzw. Spezialisierung führen. Eine zu weit getriebene Spezialisierung kann ebenfalls kontraproduktiv wirken. Denn, benötigt man für jeden Handgriff eine spezialisierte Arbeitskraft, entstehen Probleme wie Dequalifizierung, Monotonie, Entfremdung, einseitige Belastung und mangelnde Flexibilität, die sich negativ auf die Produktivität auswirken. Auch im Bereich des Tausches sowie der Abstimmung können Mängel auftreten, wenn beispielsweise die Unternehmensleitung einzelne Unternehmensbereiche nicht adäquat koordinieren und auf ein gemeinsames Unternehmensziel lenken kann. Mängel manifestieren sich somit als nicht ausgeschöpfte Produktivitätspotentiale einerseits und/oder wieder verspielte Produktivitätsgewinne andererseits. Es gilt in Unternehmen eine Unternehmensstruktur der passenden Arbeitsteilung und Spezialisierung sowie der bestmöglichen Abstimmung und des bestmöglichen Tausches $\mathrm{zu}$ realisieren (Picot/Diet//Franck, 1997).

Auch wenn in einem Unternehmen eine solche Unternehmensstruktur vorhanden ist, so ist dies keine Garantie dafür, dass in Unternehmen keine Mängel im Wirt- 
schaften auftreten. Denn das Hauptaugenmerk muss auf Mitarbeiter, die basierend auf einer solchen Unternehmensstruktur zusammenarbeiten, gelegt werden. Es kann festgestellt werden, dass sich Unternehmen zwei voneinander analytisch trennbaren Problemen gegenübersehen. Einerseits müssen Mitarbeiter koordiniert werden, was dazu führt, dass sie überhaupt die Informationen darüber haben müssen, welche Rolle innen in einer überlegenen Struktur des Wirtschaftens zukommt. Mitarbeiter müssen wissen, was sie tun müssten, um zur Erreichung der Unternehmensziele beizutragen, d.h. welche Aktivitäten und Teilbeträge sie dazu in welcher Qualität und Menge zu welchem Zeitpunkt und mit welchem Aufwand erbringen müssten. Dieses Teilproblem wird als Koordinationsproblem bezeichnet und umfasst die Überwindung des Nichtwissens seitens der Mitarbeiter hinsichtlich dessen, was zu tun ist. Ob Mitarbeiter dann die innen zugewiesenen Aufgaben auch tatsächlich erledigen, ist eine Frage, die bereits den zweiten Problembereich einleitet. So kann nicht davon ausgegangen werden, dass Mitarbeiter die formal vorgegebenen Strukturen der Arbeitsteilung usw. selbständig auch bereitwillig umsetzen. Diese Notwendigkeit, die einzelnen Akteure zur Einhaltung einer bestimmten Art der Arbeitsteilung/Spezialisierung bzw. des Tausches und der Abstimmung zu bewegen, wird als Motivationsproblem bezeichnet. Die Überwindung des Nichtwollens der Akteure wird angestrebt. Wenn Mitarbeiter ihre eigenen Ziele erfüllen können, wenn sie die an sie gestellten Aufgaben erledigen, so wird sich basierend auf der Annahme der individuellen Nutzenmaximierung auch ihre Bereitschaft erhöhen, die erwartete Leistung zu erbringen und die vereinbarten Spielregeln zu beachten. Je weniger persönliche Nutzenvorstellungen durch die formalen Leistungserwartungen erfüllt werden können, desto größer werden die Motivationsprobleme der Akteure sein (Milgrom/ Roberts, 1992). Dies kann dazu führen, dass durch die Nichteinhaltung der zugeordneten Arbeitsinhalte und -regeln einzelner Akteure auch andere Mitarbeiter zu Schaden kommen. Vor allem Situationen, in denen das Sanktionspotential anderer Akteure nicht aktivierbar ist, in denen also die Kosten der Kontrolle und der Regeldurchsetzung hoch sind, fordern zum sog. opportunistischen Verhalten heraus (Picot/Diet//Franck, 1997). 
Mithilfe von Institutionen können Unternehmen diese Koordinations- und Motivationsprobleme überwinden bzw. zu bewältigen versuchen. Erwartet man sich bei einer Begriffsdefinition von "Institution" eine präzise Ausformulierung, so kann dieser Wunsch nicht erfült werden, denn ein Zuviel an Genauigkeit wurde bewusst vermieden (Arrow, 1970).

Institutionen sind sanktionierbare Erwartungen, die sich auf die Verhaltensweisen eines oder mehrerer Individuen beziehen. Sie können sowohl einzelne, Personenmehrheiten oder alle Mitglieder einer Gesellschaft adressieren. Sie dienen jedem Einzelnen als Wegweiser bei der Aufstellung und Realisierung seiner Handlungspläne. Somit informieren Institutionen über die eigenen Handlungsmöglichkeiten und -grenzen ebenso wie über die an andere zu stellenden Erwartungen. Institutionen umschließen Regeln bzw. Normen (wie Menschenrechte, Gesetze, Sprache etc.) einerseits und korporative Gebilde (Unternehmen, Verbände, Staat etc.) andererseits. Sie wirken nicht isoliert, sondern entfalten erst innerhalb der Gesamtheit des Institutionengefüges ihre volle Wirkung (Picot/ Diet//Franck, 1997).

Als Teil der Wirklichkeit und als externes Umfeld von Organisationen, das unabhängig von der individuellen Wahrnehmung besteht und sich entwickelt, treten Institutionen in Erscheinung (Ebers/Gotsch, 1998, Williamson, 1995). Sie werden als Spielregeln der Gesellschaft oder Organisation gesehen bzw. formaler ausgedrückt als die von Individuen entwickelten Beschränkungen, die folglich das menschliche Verhalten formen und beeinflussen (North, 1990). So wird der Charakter von Institutionen als exogen gegenüber den Akteuren in Form von Regeln, Rechten, Vorschriften und Mustern, die als "constraints" und "opportunities" Handlungen eigennütziger Akteure von außen steuern, hervorgehoben. Dem beschränkenden Charakteristikum wurde eine nominalistische Betrachtungsweise komplementär zur Seite gestellt. Institutionen werden demnach nicht nur als ein externes Phänomen betrachtet, sondern die von Individuen gestaltete Wirklichkeit bedingt deren Entstehung und Fortbestand. Institutionen werden von In- 
dividuen entwickelt und verändert und als Kreationen von Menschen angesehen (North, 1990).

Im Bereich der Organisationsforschung treten Institutionen als ein System formgebundener (formaler) und formungebundener (informeller) Regeln einschließlich der Vorkehrungen zu deren Durchsetzung (Richter/Furubotn, 1996, S. 7) auf. Dadurch verfügt ein Unternehmen über ein institutionalisiertes Funktionsset, das explizit oder implizit festhält, wer für Entscheidungen in einem bestimmten Bereich in Frage kommt, welche Handlungen durchgeführt werden und in welcher Form, welche Regeln dabei zur Anwendung gelangen und welche Verfahren eingehalten werden müssen (Ostrom, 1990). Individuen behalten zwar ihre Handlungsautonomie, jedoch wird das Spielfeld durch Institutionen klar abgesteckt.

Institutionen determinieren das Verhalten der Individuen nicht, dennoch werden Akteure bei der Verfolgung ihrer Ziele die jeweils gesetzten Chancen und Risiken, Anreize und Kosten als institutionelle Handlungsbedingungen wahrnehmen und in ihren Entscheidungen berücksichtigen (Edeling, 1999). Institutionen setzen Regeln mit konstitutivem und normativem Charakter fest und definieren dadurch die Natur der Akteure und deren Handlungskapazitäten sowie des Handlungsspielraums (Scott, 1994; Hassen/Krücken, 1996).

Institutionen bieten durch einen vorgegebenen Spielraum neben einer gewissen Stabilität auch Unterstützung bei der Vermeidung von Unsicherheiten. Unternehmen arbeiten stetig daran, ihre bestehenden Ressourcen optimal zu verwenden und neue Möglichkeiten dafür mit Hilfe eines effizienten und effektiven organisationalen Arrangements zu etablieren, um in einem wettbewerbsgetriebenen Umfeld zu überleben und die Ziele von Individuen über Organisationsziele zu erreichen (vgl. Penrose, 1959). Durch eine stabile Struktur (was jedoch nicht unbedingt effizient bedeutet) werden Unsicherheiten vermindert und Institutionen schaffen Möglichkeiten, die Unternehmen zu ihrem eigenen Nutzen gebrauchen können, um besser zu überleben. Dadurch kommt es zu einem organisationalen Fortschritt, der wiederum einen institutionellen Wandel hervorruft (North, 1990). 
Eingebettet in Institutionen finden sich Organisationen, die ähnlich wie diese den menschlichen Interaktionsprozessen eine Struktur verleihen. Sie werden von Institutionen insofern unterschieden, als sie sich über individuelle und kollektive Organisationsziele definieren und von intentional handelnden Akteuren als Mitglieder getragen werden, deren Interaktion formalen und informellen Regeln unterliegt. So werden sie geformt und etabliert, um einen Zielpluralismus zu erreichen, der wiederum in der institutionellen Einbettung als realistisch zu erreichen gilt. Organisationen schließen Institutionen und die daran beteiligten Personen mit ein (North, 1990).

Individuen sehen sich nicht nur einem Set an Institutionen gegenüber, sondern sind in einer pluralen Gesellschaft in eine Vielzahl an Funktionssystemen und Organisationen inkludiert, wobei sie stets im Grunde ihr autonomes Handeln behalten. Dadurch dass Institutionen divergierende Wahrnehmungen, Wertungen, Rationalitäten ermöglichen, gewinnt ein Akteur an Handlungsspielraum (Edeling, 1999). 


\subsection{Teiltheorien der Neuen Institutionenökonomie}

Die Theorie der Neuen Institutionenökonomie setzt ihren Schwerpunkt auf Institutionen, die als sanktionierbare Erwartungen, die sich auf die Handlungs- und Verhaltensweisen eines oder mehrerer Individuen beziehen, charakterisiert werden. Im Rahmen dieser Theorie werden die Auswirkungen von Institutionen (wie z.B. Verträge, Organisationsstrukturen) auf menschliches Verhalten untersucht, wobei Möglichkeiten des effizienten Designs von Institutionen näher betrachtet werden (Picot/Diet//Franck, 1997).

Die Teiltheorien, die sich in der Neuen Institutionenökonomie erschließen, sind erstens die Theorie der Verfügungsrechte (Property-Rights Theory). Hierbei definiert das System der Verfügungsrechte in einem Wirtschaftssystem die Positionen der Wirtschafter im Hinblick auf die Nutzung knapper Ressourcen. Dem gegenüber steht die zweite große Theorie innerhalb der Neuen Institutionenökonomie die Agenturtheorie (Principal-Agency Theory), die sich mit Anreizproblemen und Fragen asymmetrischer Information befasst. Die letzte Strömung, die Erwähnung finden soll, ist die Transaktionsökonomik (Transaction Costs Theory), wobei Transaktionskosten im Zusammenhang mit einem Tauschprozess entstehen und sich ihre Höhe auf die Art und Weise der Organisation und Durchführung wirtschaftlicher Tätigkeit auswirkt.

Somit hat die Neue Institutionenökonomie erstens eine ökonomische und vertragstheoretische Perspektive auf organisationale Beziehungen gerichtet, zweitens ihren theoretischen Fokus auf Probleme hierarchischer Kontrolle und der Prinzipal-Agent-Beziehung gelegt und drittens geht sie von der universellen Existenz individuellen opportunistischen Verhaltens und der ökonomischen Relevanz "weicher Faktoren" wie Würde, Vertrauen, Fairness, Moral und dergleichen aus (vgl. Wieland, 1997).

Um sich einen strukturierten und klaren Einblick in diesen Theoriekomplex zu verschaffen, scheint es angebracht, die Ansätze, die diese Theorie prägen, ge- 
trennt voneinander darzustellen. Von Interesse zeigt sich bei der Präsentation der einzelnen Teiltheorien, welche Gestaltungsoptionen diese bieten, um einen Wissensmanagement-Ansatz zu elaborieren, der unter Berücksichtigung eines institutionellen Rahmengerüsts das Spielfeld eines Unternehmens auf eine Weise absteckt, in dem eine für das jeweilige Unternehmen optimale bzw. passende Koordination und Motivation von Mitarbeitern erfolgen kann. Der Bewältigung dieser beiden Problemfelder wird im Rahmen eines Wissensmanagement-Ansatzes eine Schlüsselrolle zugesprochen. Unternehmen zeichnen vom Willen und Wissen ihrer Mitarbeiter abhängig und ein Wissensmanagement, das für den Erfolg eines Unternehmens förderlich sein soll, sollte daher dem Individuum eine besondere Beachtung schenken. Individuen wurden als die Quelle von Wissen ausgemacht. Institutionen wirken sich auf das Verhalten von Individuen aus. Es stellt sich nun die Frage, auf welche Weise das Design von Institutionen gestaltet werden sollte, um Koordination und Motivation von Mitarbeitern gewährleisten zu können und dadurch ein für das jeweilige Unternehmen adäquates Wissensmanagement möglich zu machen.

Da bereits zahlreich Übersichtsartikel zu den Teiltheorien der Neuen Institutionenökonomie verfügbar sind (beispielsweise bei Hart/Holmström, 1987; Milgrom/ Roberts, 1988; Alchian/Woodward, 1988; Holmström/Tirole, 1989), wird die Darstellung der einzelnen Theorien auf das Wesentliche beschränkt und vor allem hinsichtlich des möglichen Beitrags zur besseren Bewältigung des Koordinationsund Motivationsproblems von Unternehmen erörtert. 


\subsubsection{Die Transaktionskostentheorie}

\subsubsection{Theoretisches Rahmenkonzept}

Die grundlegende Untersuchungseinheit der Transaktionskostentheorie ist die einzelne Transaktion (Picot/Diet//Franck, 1997). Individuen müssen Zeit und Ressourcen aufwenden, um sich Informationen zu beschaffen, wobei sie nur eine begrenzte Fähigkeit mit sich bringen, die gesammelten Informationen auch zu verarbeiten und Pläne zu formulieren. Transaktionskosten sind einerseits das Resultat dieser Ineffizienz (Furubotn/Richter, 2000), andererseits entstehen sie durch die institutionelle bzw. vertragliche Regelung der Produktion und des Austausches von Gütern und Leistungen zwischen Anbieter und Nachfrager (Williamson, 1985).

Erstmalig aufgetreten ist der Begriff der Transaktionskosten bei Coase (1937) und er gab den Anstoß dazu, sich auch mit den vertraglichen Problemen der Gestaltung von Leistungstransfers zu beschäftigen. Eine von der Neoklassik geprägte Sichtweise schätzte die Notwendigkeit von Unternehmen als gering ein, da der Markt als die effizienteste Form der Allokation galt. Dies wurde durch die Frage ${ }_{n}[\ldots]$ why is there any organisation?" von Coase pointiert hinterfragt. Er stellte die These auf, dass die Benutzung des Marktes zur Koordination von Leistungsbeziehungen keineswegs kostenlos sein könne, da es sonst nicht sinnvoll wäre, anstelle marktlicher Koordination eine solche über die Hierarchie in einem Unternehmen stattfinden zu lassen (Coase, 1937). Zu den Transaktionskosten wurden jene Ressourcen, die für Schaffung, Erhaltung, Benützung, Veränderung usw. von Institutionen oder Organisationen aufzuwenden sind, hinzugezählt.

Bei Williamson wurden dann die Ausführungen über die Transaktionskosten weitergeführt. Diese wurden als „Kosten der Inanspruchnahme des Vertragsmechanismus" definiert. Ihr Zustandekommen analysierte er anhand von Verhaltensannahmen über die beteiligten Akteure und von verschiedenen Charakteristika der Transaktionen. Es interessierte vor allem die relative Vorteilhaftigkeit unterschiedlicher institutioneller Arrangements zur Abwicklung wirtschaftlicher Trans- 
aktionen, die nicht nur über Organisationen (bzw. Hierarchien), sondern auch über den Markt oder langfristige Rahmenverträge vollzogen werden können. Mittels der Transaktionskostentheorie wird dann versucht zu erklären, warum bestimmte Transaktionen in bestimmten institutionellen Arrangements mehr oder weniger effizient abgewickelt und organisiert werden (Williamson, 1985).

Eine Möglichkeit, Transaktionskosten für Unternehmen besser handhabbar zu gestalten, liegt darin, den Teilbereich Transaktionen zu definieren. Williamson widmete sich dieser Definition und unter Transaktion wird ein Vorgang verstanden, "when a good or a service is transferred across a technologically separable interface" (Williamson, 1989, S. 142). Diese technische Definition wird insofern begründet, als die relative Effizienz von Markt und Unternehmen zu untersuchen und Gründe für die Existenz beider Allokationsmechanismen zu liefern sind. Demgegenüber steht die Definition von Commons (1934), der den Transfer von Rechten an Gütern als Transaktion definiert. "Transaction, as thus defined, are not the "exchange of commodities", in the physical sense of "delivery", they are the alienation and acquisition, between individuals, of the right of future ownership of physical things, as determined by the collective rules of society" (Commons, 1934, S. 50).

Unabhängig davon, welche Definition zugrunde gelegt und was unter Transaktion verstanden wird, führen Transaktionen zu Kosten (hierbei herrscht in den Beiträgen auf diesem Gebiet gemeinhin Einigkeit). Sie umfassen alle inner- wie außerorganisatorischen Übertragungen von Verfügungsrechten und inkludieren einerseits Markttransaktionskosten in Form von beispielsweise Such- und Informationskosten, Verhandlungs- und Entscheidungskosten sowie Überwachungs- und Durchsetzungskosten vertraglicher Leistungspflichten und andererseits Unternehmenstransaktionskosten, die beispielsweise Kosten der Einrichtung, Erhaltung oder Änderung einer Organisationsstruktur und Kosten des Betriebes einer Organisation (wie Informationskosten oder Kosten im Zusammenhang mit der physischen Übertragung von Gütern und Dienstleistungen über eine trennbare Schnittstelle) beinhalten (Williamson, 1985, S, 20ff). Die Höhe dieser Kosten 
zeichnet von drei Charakteristika abhängig: (1) vom Ausmaß, in dem die Transaktionspartner transaktionsspezifische Investitionen tätigen (asset specificity), (2) von der mit einer Transaktion verbundenen Unsicherheit (uncertainty) sowie (3) von der Häufigkeit der Transaktion (frequency) (Williamson, 1985).

Nachdem eine Transaktion als Ergebnis expliziter (ausdrücklicher) und impliziter (stillschweigender, durch entsprechendes Handeln anerkannter) Verträge durchgeführt wird, muss beachtet werden, dass in der Regel nicht von vollständigen, alle Eventualitäten berücksichtigenden Verträgen ausgegangen werden kann. Dies führt zu den der Theorie der Neuen Institutionenökonomie allgemein und der Transaktionskostentheorie im Speziellen zugrunde liegenden Annahmen über die Natur des Menschen zurück, wo von einer beschränkten Rationalität (Simon, 1991) und Opportunismus ausgegangen wird. Somit kommt es im Rahmen von Transaktionen zu ex-ante-Transaktionskosten, die durch vorvertragliche Aufwendungen entstehen, und zu nach-vertraglichen Konkretisierungen, Ergänzungen und Anpassungen der Austauschbeziehungen, die unter ex-post-Transaktionskosten zusammengefasst werden. Je nach Ausmaß der Spezifizierung und Abhängigkeit vom jeweiligen Partner gestalten sich die Produktionskosten und somit auch die Transaktionskosten einer Transaktion. Besteht eine längerfristige Beziehung zwischen den Vertragspartnern, so können diese opportunistisch im Sinne ihres eigenen Nutzens die Verträge zu ändern versuchen. Im Gegensatz dazu ist der opportunistische Handlungsspielraum in Austauschverhältnissen, im Rahmen dessen unspezifische Inputfaktoren eingesetzt werden, eingeschränkt, da ein Auflösen der Transaktion ohne größere Erlöseinbußen durchgeführt werden kann. Opportunistisches Verhalten kann somit durch die Konkurrenz anderer Anbieter oder Nachfrager wirksam unter Kontrolle gehalten werden. Die Spezifität einer Leistungsbeziehung wird durch die strategische Bedeutung relativiert, die die in einer Leistungsbeziehung koordinierten Teilaufgaben für einen oder auch für beide Transaktionspartner haben (Picot/Diet//Franck, 1997).

"Unsicherheit", das zweite Charakteristikum von Transaktionen, bezeichnet das Maß für die Vorhersehbarkeit und die Anzahl der notwendigen Änderungen der 
Leistungsvereinbarung während einer Transaktion (Picot/Diet//Franck, 1997). Sie kann einerseits aus einer "parametrischen" und andererseits aus einer "Verhaltensunsicherheit" bestehen ( $\mathrm{vgl}$. Williamson, 1985). Erstere beschreibt die Unsicherheit über die situativen Bedingungen der Transaktion und deren zukünftige Entwicklung, wobei letztere dadurch entsteht, da Vertragsteilnehmer unter Umständen ein opportunistisches Verhalten an den Tag legen. Obwohl beide Arten unterschiedliche Implikationen für die effiziente Gestaltung einer Austauschbeziehung haben, gilt in Bezug auf beide, dass mit wachsender Unsicherheit c.p. sowohl die ex-ante-Transaktionskosten als auch die ex-post-Transaktionskosten steigen (Ebers/Gotsch, 1999).

Das opportunistische Verhaltenspotential ökonomischer Akteure kann verstärkt auftreten, wenn die Transaktion durch Spezifität gekennzeichnet ist. Hierbei steht im Mittelpunkt der Betrachtung, inwiefern ein Unternehmen in der Lage ist, die mit einer Investition verbundenen Fixkosten wieder gutzumachen. Je häufiger Transaktionspartner identische Transaktionen miteinander abwickeln, desto eher lassen sich Skalen- und Synergieeffekte realisieren (vgl. Williamson, 1985, S. $60 f)$.

Um eine Transparenz in den möglichen vertraglichen Austauschbeziehungen zu schaffen und dadurch Transaktionen für die beteiligten Akteure kalkulierbarer und besser handhabbar zu gestalten, wurden etwaige institutionelle Arrangements systematisiert. Zwischen der Art eines Arrangements und der Höhe der Transaktionskosten lässt sich eine relationale Beziehung feststellen (Williamson, 1985).

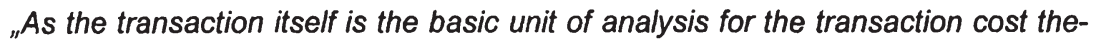
ory it has been proposed that transactions, which differ in their attributes, are aligned with organisational structures, which differ in their costs and competence, in a discriminating - mainly, transaction cost economising-way" (Williamson, 1995, S. 225). 
Einerseits können klassische Verträge geschlossen werden, die sich durch ihre Einfachheit, Kurzfristigkeit und einen marktlichen Austausch charakterisieren lassen. Mit "Einfachheit" wird darauf Bezug genommen, mit welchem Aufwand das Eingehen sowie das Auflösen einer solchen Vertragsbindung verbunden sind. So wickeln Transaktionspartner ihre Transaktionen nach fixen, ex-ante vertraglich festgelegten Regeln ab und gehen keine über den einzelnen Tauschakt hinausweisenden Verpflichtungen ein (Williamson, 1985, S. 69).

Werden andererseits die vertraglichen Regelungen und Verpflichtungen erst im Zuge der durchgeführten Transaktion bzw. den Transaktionen konkretisiert, kann das Verhalten des jeweiligen Transaktionspartners nicht vorweg klar bestimmt werden, was den Abschluss einer anderen Art von Verträgen notwendig macht, nämlich der neoklassischen Verträge. Der Identität der Akteure wird Beachtung geschenkt und je nach einer sich ändernden Datenlage steht es den Akteuren offen, die Anpassung von Vertragsleistungen zu fordern. Den Rahmenbedingungen der gegenseitigen Abhängigkeit wird insofern Rechnung getragen, als auf ein institutionalisiertes Rahmengerüst zurückgegriffen wird, das Konfliktregelungsmechanismen beinhaltet (wie beispielsweise Indexierungsklauseln, das Recht der einseitigen Vertragsauflösung, Anpassung der Preise an die tatsächliche Kostenentwicklung) (Williamson, 1985, S. 70f).

Diesen beiden Arten von Arrangements steht eine dritte Vertragskategorie gegenüber, die den diskreten Charakter der Transaktion noch weiter auflöst und sich durch Langfristigkeit und eine noch größere Offenheit bezogen auf die zu erbringenden Leistungen auszeichnet - relationale Verträge -, die für Transaktionen in Organisationen charakteristisch sind (Williamson, 1985, S. 71f).

Wird einer spezifischen Transaktion ein passendes institutionelles Arrangement zugrunde gelegt, wird diese umso effizienter organisiert und abgewickelt werden können. So ist es notwendig, sich im Vorfeld näher mit den Einflussgrößen auf die Effizienz der Abwicklung und Organisation auseinander zu setzen, die sich aus (1) der Anreizintensität, (2) den Kontrollmechanismen, (3) der Anpassungs- 
fähigkeit sowie (4) den Kosten der Etablierung und Nutzung des institutionellen Arrangements selbst zusammensetzen (Williamson, 1991).

Abhängig davon, um welche Transaktion es sich handelt, wird entschieden, ob diese über den Markt, innerhalb des Unternehmens oder in Form von Hybriden durchgeführt wird, was weiter die Gestaltung der institutionellen Arrangements beeinflusst, die wiederum das Ausmaß und die Höhe der Transaktionskosten bestimmen ( $\mathrm{vgl}$. Williamson, 1985). Die Existenz bestimmter Organisationsformen kann somit durch ihre Transaktionskosteneffizienz, die den Vergleich der Kosten alternativer Lösungen für Transaktionsprobleme darstellt, erklärt werden.

\subsubsection{Gestaltungspotentiale}

Die Transaktionskostentheorie schaffte es mit ihren ökonomischen Beiträgen, die unternehmenstheoretische Organisationsforschung zu erweitern. Nicht nur, dass die Existenz von Unternehmen begründet wurde, sondern man widmete sich vor allem den zentralen Problemstellungen wie der Frage nach Art und Ausmaß der Spezialisierung (konzeptionalisiert über Art und Ausmaß transaktions-spezifischer Investitionen) und nach der Form der Koordination arbeitsteilig erstellter Leistungen (konzeptionalisiert über alternative institutionelle Arrangements). Dadurch, dass im Rahmen dieser Theorie das Augenmerk vor allem auf Kriterien wie Effizienz, Kostenreduzierung und Leistungssteigerung gelegt wird, unterliegen alternative Formen der Abwicklung und Organisation von wirtschaftlichen Austauschbeziehungen auch einer genauen Analyse. Es kommt zu einer Analyse, wobei die einzelne Transaktion stets den Ausgangspunkt der vergleichenden Betrachtung unterschiedlicher Institutionen darstellt, die es mit Hilfe einer entsprechenden Vertragsstruktur zu organisieren gilt. Dem zugrunde wird ein den ökonomischen Theorien inhärentes Menschenbild gelegt, das von einer beschränkten Rationalität und einem opportunistischen Verhalten ausgeht, wodurch bezugnehmend auf diese Verhaltensannahmen schlussendlich der ${ }_{n} \mathrm{Homo}$ Oeconomicus" von einem "organizational man" (Williamson) abgelöst wird. So stellt 
sich die zentrale Aussage der Transaktionskostentheorie als: „Economizing on transaction costs essentially reduces to economizing on bounded rationality while simultaneously safeguarding the transactions in question against the hazards of opportunism" (Williamson, 1979, S. 245-246) dar.

Durch die Beiträge auf diesem Gebiet können Regelmäßigkeiten in den Strukturen von Organisationen und dem Verhalten ihrer Mitglieder erkannt werden und es wird ein in sich logisch konsistent aufgebauter Erklärungsansatz geboten. Dieser beschränkt sich jedoch zumindest im Rahmen der Beiträge von Williamson auf die vertikalen Strukturen von Organisationen. Somit bedarf es einer Ausdehnung des Erklärungsansatzes um die horizontale Dimension. Dadurch kann die Schwärze der "black box“ Unternehmen erhellt werden und die Erklärung potentieller Quellen unternehmerischer Wettbewerbsvorteile unterstützt werden. Die Kontraktproblematik der horizontalen Leistungsorganisation in den Mittelpunkt stellend wurde somit die Teamproduktion als Ausgangsmodell zu einem generellen Unternehmensmodell weiterentwickelt (Alchian/Demsetz, 1972). Zwei Fragestellungen liegen dem zugrunde: (1) Können Erlöse einer Spezialisierung und Kooperation besser innerhalb eines Unternehmens oder über den Markt realisiert werden und (2) muss eine Theorie der Unternehmung in der Lage sein, die Struktur von Organisationen zu erklären? Die traditionelle Vorstellung von Unternehmen, die mit Hilfe von Macht- und Autoritätsstrukturen sowie disziplinierenden Maßnahmen Ziele durchsetzen, wird überwunden und „[it] [das Unternehmen, Anm. S.M.] has no power of fiat, no authority, no disciplinary action any different in the slightest degree from ordinary market contracting between any two people" (Alchian/Demsetz, 1972, S. 778). So werden Unterschiede zwischen Unternehmen und Märkten nicht in den vorhandenen Sanktionsmechanismen oder der Dauer der Vertragsverhältnisse ausgemacht, sondern sie bestehen durch das Vorhandensein von Kooperations-vorteilen, die nur innerhalb von Teams realisiert werden können. Es wird die Verhaltensannahme zugrunde gelegt, dass, solange ein direkter und messbarer Zusammenhang zwischen der Inputleistung und dem Arbeitsergebnis besteht, ein Individuum bestrebt ist, einen qualitativ und quantitativ hohen Arbeitseinsatz zu Jeisten (vgl. Alchian/Demsetz 1972 ) Dieses 
ursprüngliche Modell wurde dann von Alchian in Zusammenarbeit mit verschiedenen Autoren (beispielsweise Alchian/Woodward, 1987; Alchian, 1984) weiterentwickelt und ist auch als die Koalitionstheorie bekannt.

Doch nicht nur die mangelnde Integration der horizontalen Dimension bei Williamson gab anderen Autoren Anlass zu einer Weiterentwicklung der Transaktionskostenstheorie, sondern auch den zugrunde liegenden Verhaltensannahmen war ein Kritik- und Ergänzungspotential inhärent. Kritische Stellungnahmen zur Transaktionskostentheorie finden sich beispiels-weise bei Ebers/Gotsch (1999), Edeling/JannMagner (1999), Ortmann/Dydow/Türk (1997) oder Ghoshal/Moran (1996).

Die Charakteristika der einzelnen institutionellen Arrangements lassen noch einen breiten Interpretationsspielraum übrig, da die relativ einfache Konzeptionalisierung sich lediglich bei Aussagen zu einfachen institutionellen Formen als passend erweist und da für komplexere Formen noch ein analytisches Instrumentarium entwickelt werden muss. Trotz einer Praktikabilität der Ausführungen und einer Bewährung der Thesen der Transaktionskostentheorie in empirischen Untersuchungen lassen sich die unterschiedlichen Strukturtypen in der Praxis nicht klar voneinander getrennt wieder entdecken. Dennoch bietet diese Theorie pragmatisch verwertbare Gestaltungsanregungen, mit Hilfe derer Transaktionskosten reduziert werden können (vgl. Ebers/Gotsch, 1999; Wieland, 1999).

Im Zuge der Elaborierung eines Wissensmanagement-Ansatzes wird das Hauptaugenmerk auf die Bewältigung des Koordinations- und Motivationsproblems von Unternehmen gelegt. In diesem Zusammenhang bietet die Transaktionskostentheorie brauchbare Ideen und Ausführungen. Optimierungsstrategien ökonomischer Akteure können zwei Ansatzpunkte wählen (Picot/Diet//Franck, 1997): eine möglichst produktive Arbeitsteilung bzw. Spezialisierung und möglichst "reibungslose" Tausch- bzw. Abstimmungsprozesse. Diese beiden Ansatzpunkte werden auch in der Transaktionskostentheorie adressiert, wobei sie sich im Rahmen ihrer Erklärungs- und Gestaltungsaussagen vor allem auf die Tausch- bzw Abstim- 
mungsphase des Wirtschaftens konzentriert, jedoch in Teilbereichen sehr wohl auch auf die Frage der "richtigen" Arbeitsteilung und Spezialisierung eingeht. Die von Unternehmen zu verrichtenden Aufgaben werden in Teilaufgaben gegliedert, wobei darauf geachtet werden sollte, dass die Transaktionskosten der im Aufgabenerfüllungsprozess auftretenden Austauschvorgänge möglichst gering ausfallen. Im Zuge eines Wissensmanagements kann sich dies in der Form darstellen, dass zwischen den einzelnen Aufgabenträgern ein möglichst geringer Wissenstransfer vonnöten ist. Derartige Leistungen, die weiterverwendbar sind, ohne dass ein Rückgriff auf das für ihre Erstellung nötige Wissen erforderlich ist, helfen die unternehmensinternen Transaktionskosten niedrig zu halten (Picot) Diet//Franck, 1997). Die Idee der Berücksichtigung der innerhalb eines Unternehmens entstehenden Transaktionskosten stellt sich als dienlich für ein Wissensmanagement dar. Denn mithilfe von Organisationsstrukturen werden Aufgabenbereiche von den jeweiligen Akteuren eines Unternehmens abgesteckt. Sie setzen den Handlungsspielraum der Unternehmensmitglieder fest. Der Grad der Spezialisierung und die Art und Weise, wie ein unternehmensinterner Austausch erfolgt, sollte von den Organisationsstrukturen in einer Form abgebildet werden (lässt man die informellen Interaktionsprozesse, die in einem Unternehmen stattfinden, an dieser Stelle gänzlich außer Acht), sodass sich dies für ein Unternehmen als "optimal", d.h. in diesem Zusammenhang als "transaktionskostensenkend", darstellen würde.

Eine einseitige Betrachtung der Austauschprozesse innerhalb eines Unternehmens anhand der zugrunde liegenden Transaktionskosten greift jedoch zu kurz. Denn auch wenn sich eine Aufgabenspezialisierung mit einer Senkung von Transaktionskosten positiv korreliert (vgl. Picot/Diet//Franck, 1997, S. 74), so sollte in einem nächsten Schritt genauer untersucht werden, welche Auswirkungen dies auf ein Wissensmanagement hat, was unter anderem Gegenstand des in der vorliegenden Arbeit entworfenen Wissensmanagement-Ansatzes darstellen wird. 
Die Koordinations- und Motivationsproblematik innerhalb eines Unternehmens wird ebenfalls durch die der Transaktionskostentheorie zugrunde gelegten Verhaltensannahmen adressiert. Der Risikoneutralität wird in der vorliegenden Arbeit keinerlei besondere Beachtung geschenkt. Vielmehr interessieren das opportunistische sowie nutzenmaximierende Verhalten von Unternehmensakteuren. Opportunismus soll jedoch nicht bedeuten, dass grundsätzlich davon ausgegangen wird, dass die Motivationsstrukturen von Akteuren auf das Streben nach Geld, Gütern und Leistungen reduziert werden. Vielmehr gilt es, auf der Annahme zu basieren, dass grundsätzlich mit einem opportunistischen Verhalten von Mitarbeitern gerechnet werden und ein Unternehmen entsprechende Vorkehrungen treffen sollte. Dieses Vorsichtsprinzip begründet sich aus der begrenzten Rationalität von Individuen, aufgrund der ein Akteur nicht mit Sicherheit voraussehen kann, welcher Transaktionspartner und wann dieser opportunistisch handeln wird (vgl. Ebers/Gotsch, 1999, S. 243-244). Ein Wissensmanagement, das seinen Fokus auf das Individuum legt und es als Quelle von Wissen ansieht, sollte daher, wenn auf Annahmen der Transaktionskostentheorie rekurriert wird, einerseits die zugrunde liegenden Verhaltensannahmen sowie andererseits die Senkung von Transaktionskosten berücksichtigen. 


\subsubsection{Die Theorie der Verfügungsrechte}

\subsubsection{Theoretisches Rahmenkonzept}

Verfügungsrechte definieren das legitime Ausmaß der Nutzung einer Ressource, für das der jeweilige Inhaber die Rechte innehat. Sie spezifizieren institutionalisierte Verhaltensnormen, inwieweit eine Ressource genutzt werden kann und inwiefern die Einhaltung von diesen Normen allgemein erwartet und deren Verletzung allgemein sanktioniert wird. Der Wert eines Gutes wird hierbei nicht allein durch dessen physikalische Eigenschaften determiniert, sondern auch durch die ausübbaren Handlungs- und Verfügungsrechte beeinflusst. Somit wird im Rahmen der Theorie der Verfügungsrechte untersucht, welche Auswirkungen unterschiedliche Formen der Gestaltung und Verteilung von Verfügungsrechten auf das Verhalten ökonomischer Akteure und auf die Faktorallokation haben, und zum anderen, wie sich die Entstehung von Verfügungsrechten, ihre Verteilung und ihr Wandel erklären lassen. Zu den Begründern und Vertretern dieses Theoriezweiges werden beispielsweise Coase (1937), Alchian (1961; 1965; 1973), Demsetz (1964; 1967), Alchian/Demsetz (1972), Furubotn/Pejovich (1972; 1974), De Alessi (1980) oder North (1981) gezählt (Ebers/Gotsch, 1999).

Die Ausführungen dieser Theorie basieren auf den Grundannahmen von Akteuren mit einem nutzenmaximierenden Verhalten, dem Konzept der Verfügungsrechte sowie der Annahme, dass die Spezifizierung, Übertragung und Durchsetzung von Verfügungsrechten Transaktionskosten verursacht. Nutzenmaximierende Akteure streben jedoch nicht nur an, ihren Nutzen in Bezug auf materielle Güter zu maximieren, sondern vielmehr auch immaterielle Werte zu verwirklichen, wie beispielsweise Prestige, Selbstverwirklichung oder Macht (Ebers/ Gotsch, 1999). Die Verfügungsrechte selbst müssen immer in Bezug auf den innen inhärenten institutionellen Handlungsrahmen verstanden werden, der sich sowohl auf rechtlicher, ökonomischer und sozialer Ebene darstellt und in einer Gruppe oder Gesellschaft als gemeinhin akzeptiert gilt. Näher spezifiziert werden die Verfügungsrechte durch Einzelrechte, die das Recht (1) die Ressource zu nutzen (usus), (2) die Erträge einzubehalten (usus fructus) (3) ihre Form oder 
Substanz zu ändern (abusus) sowie (4) alle oder einzelne der vorstehenden Verfügungsrechte auf andere zu übertragen (Übertragungsrecht) mit einschließen (Alchian/Demsetz, 1972).

Die Theorie der Verfügungsrechte ist eng mit der Transaktionskostentheorie verknüpft, da sie vor allem auf den Werken von Coase und Williamson aufbaut, und sollte nicht ohne diese näher erläutert werden. Beide haben gemeinsam, dass sie einerseits Anreize und andererseits Beschränkungen für das Verhalten und die Allokationsentscheidungen von ökonomischen Akteuren setzen. Die Nutzung von Ressourcen und die damit einhergehenden Kosten stehen im Mittelpunkt der Betrachtungen. So kommt es, dass die Theorie der Verfügungsrechte die Art und Verteilung der Verfügungsrechte über eine Ressource näher bestimmt und auch die Personen definiert, denen bestimmte Nutzungsmöglichkeiten einer Ressource offen stehen. Ergänzend dazu positioniert sich die Transaktionskostentheorie, da die Kosten, die den jeweiligen Akteuren bei der Nutzung einer Ressource entstehen, davon abhängig sind, welche Kosten der Bestimmung, Übertragung und Durchsetzung der Verfügungsrechte auftreten (Furubotn/Pejovich, 1972).

Basierend auf der verhaltenstheoretischen Annahme, dass Individuen danach trachten, ihren Nutzen zu maximieren, wird die Kernaussage dieses Theoriestranges definiert. Bei gegebenen institutionellen Rahmenbedingungen werden solche Formen der Ressourcennutzung gewählt, die den Nettonutzen der Akteure maximieren helfen. Je nachdem, welche Verfügungsrechte über eine Ressource bestehen, desto unterschiedlicher gestaltet sich das Ausmaß der möglichen Nutzung dieser Ressource und somit das Ausmaß der möglichen Maximierung des Nutzens eines Akteurs. Je höher sich in einem solchen Zusammenhang die Transaktionskosten darstellen, desto niedriger fällt wiederum der zu maximierende Nettonutzen aus (vgl. Ebers/Gotsch, 1999).

Durch die Verdünnung von Verfügungsrechten, worunter das eingeschränkte Ausmaß ausübbarer Handlungs- und Verfügungsrechte verstanden wird, und von positiven Transaktionskosten kann jedgch nicht von einer optimalen Faktoralloka- 
tion ausgegangen werden. Verfügungsrechte können einerseits verdünnt werden, da nicht alle Rechte gemeinsam existieren bzw. dem gleichen Akteur zugeordnet sind und andererseits können Rechte gleichzeitig auf mehrere Personen verteilt sein. Je vollständiger die Verfügungsrechte einem Handelnden zugeordnet sind, desto effizienter wird er diese einsetzen. Dieser kausale Zusammenhang kommt durch die zugrunde gelegte Verhaltensannahme der individuellen Nutzenmaximierung zustande. Vor dem Hintergrund dieser Ausführungen sind aus alternativen Verfügungsrechts-Arrangements immer jene zu empfehlen, bei denen es zu einer möglichst vollständigen Zuordnung der Rechte auf Akteure kommt. Die Verwirklichung dieses Idealzustands scheitert jedoch an der Existenz von Transaktionskosten (Picot/Diet//Franck, 1999).

Werden optimale Verfügungsrechtsstrukturen etabliert, müssen Transaktionskosten berücksichtigt werden, die für deren Bestimmung und Durchsetzung anfallen (De Alessi, 1990; Demsetz, 1967). Entscheidungen über strukturelle Maßnahmen werden jedoch nicht nur abhängig von der jeweiligen Allokationseffizienz getroffen, sondern persönliche Werteinstellungen und individuelle Präferenzen beeinflussen diese Entscheidungen stark (De Alessi, 1980).

Wird ein Unternehmen als "a nexus of contracts“ (Jensen/Meckling, 1976) betrachtet, so positionieren sich unterschiedliche Formen von Organisationsstrukturen auf einem Kontinuum von vertraglichen Beziehungsgeflechten, sog. "standard form contracts" (Jensen/Meckling, 1976). Diese verschiedenen Unternehmensverfassungen nehmen einen großen Forschungsbereich innerhalb der Theorie der Verfügungsrechte ein (vgl. Picot, 1981; De Alessi, 1980; Furubotn/ Pejovich, 1972). Beispielhaft wurde einerseits die Unternehmung des jugoslawischen Typs, andererseits die Publikumsgesellschaft analysiert, wobei letzterer ein zentrales Forschungsinteresse zugesprochen wurde (Ebers/Gotsch, 1999). Hierbei werden vor allem die unterschiedlichen Verfügungsrechtsstrukturen zwischen einer Eigentümer-Unternehmung, wo sämtliche Verfügungsrechte über das Vermögen der Unternehmung in der Hand der Kapitaleigner konzentriert werden, und einer Publikumsgesellschaft, wo die Kapitaleigner die Rechte am Ertrag und 
das Übertragungsrecht besitzen und die Manager über die Nutzungs- und Änderungsrechte an den Ressourcen der Unternehmung verfügen, festgehalten und untersucht.

Auch die Analyse von in den verschiedenen Organisationsverfassungen vorherrschenden Mitbestimmungs- und Arbeitnehmerbeteiligungsstrukturen (Alchian/ Demsetz, 1972), ökonomische Analysen des Rechts oder wirtschafts-historische Analysen (North/Thomas, 1973; North, 1981, 1990) werden im Rahmen dieser Theorie durchgeführt. Zusätzlich bietet sie auch einen Erklärungsansatz für die Entstehung und Funktion von Organisationen (Jensen, 1983; Gäfgen, 1984) und vor allem innovationsfördernde Strukturen werden hinsichtlich ihrer Allokationseffizienz einer näheren Betrachtung unterzogen (Picot/Schneider, 1988).

\subsubsection{Gestaltungspotentiale}

In der Theorie der Verfügungsrechte kommt es (wie bereits bei der Transaktionskostentheorie festgestellt wurde) zu einem ersten Ausleuchten der "Black Box" Unternehmen. Aufbauend auf den Annahmen des methodologischen Individualismus wird ein Unternehmen durch die Handlungsbeiträge von Individuen konzipiert. Diese besitzen unterschiedliche Präferenzordnungen und verfolgen die verschiedenartigsten Zielsetzungen. Dadurch werden auch unterschiedliche Verfügungsrechtsstrukturen gebildet, die sich in Form von Unternehmen als differenzierte, vielseitig gestaltbare und vertraglich begründete Institutionen manifestieren. Diese weisen folglich auch variierende Anreiz- und Verhaltenswirkungen auf. Beim Versuch, die Auswirkungen, die Entstehung und den Wandel von Verfügungsrechtsstrukturen zu erklären, erheben sich aufgrund der theoretischen Konzeptionalisierung der Theorie der Verfügungsrechte eine Reihe von Problemen. Die empirisch beobachtbare große Vielfalt und Differenziertheit von Verfügungsrechtsstrukturen konnte theoretisch-konzeptionell und empirisch-operational nicht erfasst werden. Nicht nur, dass die Verfügungsrechtsstrukturen selbst mangelhaft präzisiert wurden, auch kam es bei der Spezifizierung der beiden 
anderen Hauptbereiche zu nicht vollständigen Ausführungen: Zum einen wird die zu maximierende Nutzenfunktion von Individuen nur selten in einschlägigen Arbeiten präzise ex-ante dargestellt (De Alessi, 1990); zum anderen werden die Transaktionskosten nur unzulänglich definiert. Dass exogene Variablen kaum eine Berücksichtigung in diesem Theoriegebäude finden und auch nicht theorieimmanent erklärt werden können, stellt ein weiteres Kritikpotential dar. So muss im Zuge der Erklärung der Entstehung und des Wandels von Verfügungsrechtsstrukturen oftmals auf theorieexogene Variablen sozialer, kultureller, politischer oder technischer Natur zurückgegriffen werden (Kritik an der Theorie der Verfügungsrechte findet sich bspw. bei Demsetz, 1967; Kieser, 1988).

Die theoretischen Erklärungen im Rahmen der Theorie der Verfügungsrechte bieten als Basis für die Entwicklung eines Wissensmanagement-Ansatzes dennoch brauchbare Grundannahmen und Erläuterungen. Die Theorie der Verfügungsrechte bezieht sich in ihren Ausführungen auf die Institutionenebene, soweit es um die Ausgestaltung alternativer Rechteverteilungen geht. Auf jeder Institutionenebene sind jeweils nur die entscheidungsrelevanten Transaktionskosten und Wohlfahrtsverluste zu berücksichtigen (Picot/Dietl/Franck, 1999).

Unbeantwortet bleibt an dieser Stelle jedoch die Frage, welche vertraglichen Rahmenbedingungen in welchen Situationen gewählt werden sollen, damit einerseits Individuen ihren Nutzen maximieren können und andererseits für Unternehmen eine optimale Ressourcenallokation mit einhergehender Optimierung von Effizienz erreicht werden kann.

Die Organisationsempfehlung der Theorie der Verfügungsrechte lautet, Handlungs- und Verfügungsrechte in der Weise zu verteilen, dass möglichst vollständige Rechtebündel mit der Nutzung ökonomischer Ressourcen verbunden und dem Handelnden zugeordnet sind, wo immer die Transaktionskosten dieses erlauben (Picot/Dietl/Franck, 1999, S. 63). Eine Koordination von Mitarbeitern erfolgt über die in einem Unternehmen definierten Vertragsrechte. Es werden tayloristische Arbeitsstrukturen relativient und M̈berwunden. Ganzheitlichkeit und der 
Versuch, Aufgabenträger mit den zur Lösung ihrer Aufgabe notwendigen Entscheidungsrechten auszustatten und an den Folgen ihrer Entscheidungen zu beteiligen, treten an die Stelle einer strikten Trennung dispositiver und ausführender Tätigkeiten sowie einer starken funktionalen Segmentierung. Im Idealfall werden Kompetenz (Entscheidungsrecht), Aufgabe und Verantwortung (Beteiligung an den Entscheidungsfolgen) jeweils einer Person vollständig zugeordnet (Picot/Diet//Franck, 1999).

Auf organisationaler Ebene gestaltet sich eine solche Herangehensweise in Form eines modularen Unternehmens, das als ein Netzwerk sich selbst steuernder Gruppen definiert ist. So werden unabhängige Aufgabensegmente, die von möglichst autonomen Gruppen ganzheitlich erfüllt werden, geschaffen. Handlungsrechte bzw. Ausführungskompetenzen, die in funktional und hierarchisch organisierten Unternehmen horizontal breit verteilt sind, werden prozessbezogen in den Modulen konzentriert. Entscheidungskompetenzen werden gleichzeitig in vertikaler Richtung vom Management auf untere, prozessnähere Ebenen verlagert ( $\mathrm{Pi}$ cot/Diet//Franck, 1999).

Die Konzeptionalisierung von Organisationen, Verfügungsrechte und das nutzenmaximierende Individuum als Ausgangspunkt nehmend, durch die Theorie der Verfügungsrechte ermöglicht, die Spezialisierung und Arbeitsteilung in einem Unternehmen in einer Form zu gestalten, dass die Verfügungsrechte richtungsweisend für die Koordination von Mitarbeitern wirken. Sicherlich treten in einem Unternehmen Interaktionsprozesse auf, die auf einer informellen Ebene stattfinden, sich somit für Verfügungsrechte als unzugänglich bzw. nur schwer zugänglich gestalten.

Jedoch im Rahmen des in der vorliegenden Arbeit zu elaborierenden Wissensmanagement-Ansatzes sollen genau die Interaktionsprozesse innerhalb eines Unternehmens adressiert werden, die sich nicht einer bewussten und aktiven Steuerung durch das Management entziehen. Der Handlungsspielraum eines Individuums wird durch dessen Verfügungsrechte in einem Unternehmen, die 
sich in den Organisationsstrukturen institutionalisiert haben, einerseits eingeschränkt. Andererseits entfaltet sich dadurch auch ein Gestaltungsspielraum für das jeweilige Individuum. 


\subsubsection{Die Agenturtheorie}

\subsubsection{Theoretisches Rahmenkonzept}

Die Agenturtheorie rückt ihr Interesse ganz auf die Institution des Vertrags und seine Rolle in Austauschbeziehungen zwischen einem Auftraggeber ("Prinzipal") und einem Auftragnehmer („Agent"). Durch das Abschließen eines Vertrags kommt es zu einer Agency-Beziehung, wobei darunter "a contract under which one or more persons (the principal/s) engage another person (the agent) to perform some service on their behalf which involves delegating some decision making authority to the agent" (Jensen/Meckling, 1976, S. 212) verstanden wird. In Organisationen finden sich solche Beziehungen beispielsweise zwischen Arbeitgeber und Arbeitnehmer, Eigentümer und Geschäftsführer, Aufsichtsrat und Vorstand, Vorstand und Führungskraft, Fremdkapitalgeber und Geschäftsführer oder Vorgesetzten und Untergebenen (Picot, 1990, S. 8). Konstitutiv für das Vorliegen einer sogenannten Principal-Agent-Beziehung ist, dass die Handlungen des Auftragnehmers (des "agent") nicht nur sein eigenes Wohlergehen, sondern auch das Nutzenniveau des Auftraggebers (des "principal") beeinflussen (Picot/Dietl/Franck, 1997). Die Austauschbeziehungen werden eingegangen, da der Prinzipal zur Realisierung seiner Interessen bestimmte Aufgaben und Entscheidungskompetenzen auf der Basis einer Vereinbarung an einen beauftragten Agenten, der für seine Dienste eine Vergütung erhält, überträgt. So kann er sich das Spezialwissen und den dadurch vorhandenen Informations-vorsprung des Agenten für seine eigene Aufgabenerfüllung und seine Zielsetzungen zunutze machen. Je weniger Einblick er jedoch in die Motive, die Handlungsmöglichkeiten und das faktische Leistungsverhalten des Agenten hat, desto größer ist sein Risiko, dass der Agent nicht nach seinem Ermessen bzw. nach den Auflagen des Auftrags handelt, sondern die eigenen Interessen zum Nachteil des Prinzipals verfolgt. Das Problem, wie ein Prinzipal vertraglich sicherstellen kann, dass der Agent eine Leistung erbringt, die der Vereinbarung entspricht, und welche Instrumentarien dem Prinzipal hierbei zur Verfügung stehen, bekundet das Hauptinteresse der Agenturtheorie (Fama/Jensen, 1983a; Fama, 1980; Jensen/ Meckling, 1976). 
Wer jeweils die Rolle des Prinzipals und wer des Agenten einnimmt, kann häufig nur situationsbezogen entschieden werden. Ein und dieselbe Person kann sowohl Prinzipal als auch Agent sein. Auch können sich zwischen denselben Akteuren mehrere Principal-Agent-Beziehungen überlappen. Vor dem Hintergrund dieser Ausführungen lassen sich Unternehmen als Geflechte ineinander verschränkter Principal-Agent-Beziehungen sehen (Picot/Diet//Franck, 1997). Dabei baut diese Theorie auf drei Grundgedanken auf. Einerseits wird die Organisation als "nexus of contracts" (Jensen/Meckling, 1976) verstanden und legt somit ein vertragstheoretisches Organisationskonzept zugrunde. Andererseits findet sich auch in dieser Strömung der Neuen Institutionenökonomie die Verhaltensannahme eines nutzenmaximierenden Individuums mit beschränkter Rationalität wider. Dieser werden eine ungleiche Informationsverteilung sowie Interessensunterschiede hinzugefügt und es wird der Risikoneigung der Akteure besondere Beachtung geschenkt. Drittens wird die Annahme unterlegt, dass mit einer optimalen Gestaltung von Verträgen die Berücksichtigung von Agenturkosten einhergehen muss.

Verträge, seien es implizite oder explizite, regeln den inter- und intraorganisationalen ökonomischen Austausch. Auf verbindliche Weise werden Zuständigkeiten, Rechte und Pflichten sowie die Zuteilung des Ertrages festgelegt. Diese "rules of the game" (Fama/Jensen, 1983a, S. 302-303; Fama, 1980; S. 289) lassen sich jedoch nicht vollständig bestimmen. Auf Grund von unvollständigen Informationen, auf Grund der Unbestimmtheit zukünftiger Umstände und der begrenzten Möglichkeiten der Antizipation von Kosten können Verträge unvollständig sein. Akteure sind sich dieser Tatsache sehr wohl bewusst und so werden in die Vertragskonditionen entsprechende Anreiz-, Kontroll- und Informationsmechanismen eingearbeitet, die den Unbestimmtheiten und Problemen der zukünftigen Auftragsbearbeitung entgegenwirken sollen. Das vertragstheoretische Organisationskonzept ist mit der Vorstellung verbunden, dass sich die gesamte Organisation mitsamt ihren Verbindungen zur Umwelt in Vertragsbeziehungen zwischen Individuen desaggregieren lässt. Somit wird die gemeinhin in der Organisationstheorie übliche Vorstellung der Trennung von OOrganisation" und "Umwelt" über- 
wunden: Organisationen sind lediglich "legal fictions which serve as a nexus for a set of contracting relationships among individuals" (Jensen/Meckling, 1976, S. 310).

Um die Hauptaussagen dieser Theorie näher zu erläutern, bedarf es jedoch vorweg einer klaren Spezifizierung der zugrunde gelegten Verhaltensannahmen, die denen der Transaktionskostentheorie weitgehend entsprechen. Die transaktionskostentheoretische Annahme begrenzter Rationalität soll zum Ausdruck bringen, dass ökonomische Akteure außer Stande sind, ${ }_{n}$ perfektes" Wissen über die Welt und ihre Gegebenheiten zu erlangen. Den gleichen Tatbestand - die Fehlerhaftigkeit menschlicher Erkenntnisse - bringt die Agenturtheorie durch die Annahme unvollständiger Information zum Ausdruck. Weiters liegt beiden Ansätzen die Annahme individueller Nutzenmaximierung zugrunde. In der Transaktionskostentheorie wird in diesem Zusammenhang besonders auf die Perspektive des Opportunismus eingegangen. Wird diese auf die Agenturtheorie übertragen, so muss der Prinzipal damit rechnen, dass der Agent Verhaltensspielräume auch dann zu seinen eigenen Gunsten nutzt, wenn er dem Prinzipal dadurch bewusst schadet (Picot/Diet//Franck, 1997).

Im Zuge einer individuellen Nutzenmaximierung achten Individuen auf ihre Präferenzstrukturen. Diese setzen sich aus einer Vielzahl an Zielen zusammen und schließen einerseits monetäre und andererseits immaterielle Werte mit ein. Da Individuen selbst nach ihrem Ermessen rational zu handeln versuchen und sie das auch abhängig von der Situation, in der sie sich befinden, tun, erwarten sie eine dementsprechende rationale Handlungsorientierung auch bei ihrem Vertragspartner. Das Verhalten der Beteiligten kann somit grundsätzlich antizipiert und durch vertragliche Vereinbarungen im Voraus gesteuert werden. Durch die divergierenden Präferenzstrukturen und die Ungleichverteilung von Informationen werden die Vertragspartner jedoch in den Austauschbeziehungen unterschiedlich auftreten und beispielsweise auch ein differierendes Risikoverhalten aufweisen. So wird zumeist dem Prinzipal Risikoneutralität und dem Agenten Risikoabneigung unterstellt. Auf Grund der individuellen Nutzenmaximierung seitens jedes 
Vertragspartners können unumstößlich Zielkonflikte auftreten, was auch den Bedarf an "governance mechanisms" wie Steuerungs- und Kontrollmechanismen intendiert (vgl. Ebers/Gotsch, 1999).

Wäre für beide Seiten einer Principal-Agent-Beziehung eine kostenlose Informationsbeschaffung möglich, dann gäbe es angesichts vollkommener Information bzw. Sicherheit keinerlei ökonomische Probleme. Aufträge könnten so erteilt bzw. Entscheidungen so delegiert werden, dass stets die produktivsten Strukturen der Arbeitsteilung und Spezialisierung realisiert würden. Spielräume für vertragsabweichendes Verhalten wären nicht gegeben, ohne dass der Gegenüber dies zu verhindern wüsste (Picot/Diet//Franck, 1997). Diese Situation findet sich in der Realität nicht wieder. Unvollständige und ungleich verteilte Informationen führen zu Abweichungen, die sog. Agenturkosten mit sich bringen, wobei diese sich einerseits aus Steuerungs- und Kontrollkosten und andererseits aus Garantiekosten sowie Residualkosten zusammensetzen. Diese Kosten drücken die Differenz einer Leistungserstellung durch einen Agenten bei vollständiger Information beider Partner einerseits und einer Leistungserstellung bei ungleicher Informationsverteilung andererseits aus (Jensen/Meckling, 1976, S. 308).

Die Ungleichverteilung von Informationen nimmt ein Hauptinteresse im Rahmen der Agenturtheorie ein und wird in Form des "hidden information bzw. adverse selection" als ein Agenturproblem behandelt. Sie stellt jedoch nicht das einzige Problem dar, sondern sie wird durch das des "hidden action bzw. moral hazard" ergänzt (Arrow, 1985), wobei sich diese im Laufe einer Austauschbeziehung abhängig von der jeweiligen Auftragsphase verändern.

Im Rahmen der Delegation von Entscheidungen an den Agenten steht diesem ein für inn grundsätzlich vorteilhafter Entscheidungs- und Handlungsspielraum zur Verfügung. Einerseits verfügt ein Agent vor dem Vertragsabschluss über Informationen, zu denen der Prinzipal keinen Zugang hat, und er kann sie bereits im Vorfeld seiner Auftragstätigkeit für seinen strategischen Vorteil und zuungunsten des Prinzipals einsetzen. Dies wirkt sich für den Prinzipal als ein Problem 
aus, da er teilweise zu wenig dafür sensibilisiert ist, die Selbstdarstellung eines Agenten als täuschend oder unvollständig zu empfinden. Auch kann ein Prinzipal vielleicht die Handlungen des Agenten beobachten, jedoch ist er nicht in der Lage, diese zu beurteilen. Der fehlende Zugang des Prinzipals zu bestimmten Kontextinformationen kann einer Bewertung der Handlungen des Agenten im Wege stehen ("hidden information") (vgl. Picot/Diet//Franck, 1997).

Andererseits verfügt der Prinzipal auch während der Auftragsabwicklung nur über eine beschränkte Einsicht in die Handlungsmöglichkeiten eines Agenten, sodass es diesem möglich ist, verschiedene Vorgehensweisen (Mittel) zur Zielerreichung und somit auch einen leichteren Weg zur Auftragsabwicklung zu wählen, als den, der Gegenstand der Austauschbeziehung ist und der auch entlohnt wird („hidden action"). Der Prinzipal kennt zwar das Handlungsergebnis, weiß aber nicht, inwieweit dieses auf Anstrengungen des Agenten und inwieweit es auf exogene Faktoren zurückzuführen ist. Die beschränkte Beobachtbarkeit des Verhaltens des Agenten verleitet diesen dazu, weniger Leistung anzubieten als nötig („shirking") oder Ressourcen eigennützig zu verwenden, um seine Lebensqualität zu erhöhen ("fringe benefits"). Auf Grund unvollkommener Koordination, die wegen Interessensunterschieden und einer Ungleichverteilung an Informationen auftreten wird, ist mit einem suboptimalen Ergebnis der Auftragsbearbeitung zu rechnen (vgl. Ebers/Gotsch, 1999; Picot/Diet//Franck, 1997).

Mittels "governance mechanisms" soll den Agenturproblemen begegnet und die Agenten zu einem auftragsmäßigen Leistungsverhalten veranlasst werden. Zum Beispiel lässt sich über eine Ergebnisbeteiligung des Agenten ein Commitment über die genauen Zielvorstellungen und deren Erfüllung herstellen, wodurch die Präferenzen und Interessen der Austauschpartner näher aneinander gerückt werden und wodurch der Anreiz für den Agenten steigt, im Sinne des Prinzipals zu handeln. Die vom Agenten erbrachte Leistung muss jedoch in einem klaren Zusammenhang mit dem Ergebnis des Auftrags stehen und nicht so sehr beispielsweise von Umwelteinflüssen abhängig zeichnen, dass der Leistungsbeitrag des Agenten nicht mehr messbar ist. Der Agent würde sich dann in einer Position 
wieder finden, in der der Ausgang des Vertrags unsicher ist. Er hätte somit ein gewisses Risiko zu tragen, das er sich im Gegenzug entlohnen lassen würde. Dies würde wiederum den Prinzipal in eine schlechtere Ausgangslage versetzen. Die optimale Gestaltung von ergebnisorientierten Verträgen müsste somit im Rahmen eines Kompromisses bei der Verteilung der Ergebnisse und des Risikos gelöst werden. Agenturprobleme werden teilweise auch anders zu lösen versucht, indem in der Rolle des Prinzipals das Verhalten des Agenten direktiv gesteuert wird. Mit Hilfe von Verhaltensnormen, die vertraglich vereinbart, deren Einhaltung kontrolliert und deren Verletzung sanktioniert wird, kann sichergestellt werden, dass im Sinne der Interessen des Prinzipals gehandelt wird und eine gute Leistungserfüllung stattfindet. Derartige Verhaltenskontrollen stoßen auf Grenzen der Durchsetzbarkeit und werden auf Grund mangelnder positiver Anreize zu einer besseren Leistungserfüllung durch den Agenten nur am Rande behandelt. Ein weitaus größeres Augenmerk wird darauf gelegt, Mechanismen zu etablieren, die positive Anreize schaffen. Im Zuge einer Verbesserung des Informationssystems, im Rahmen dessen sich ein Prinzipal ausbilden und mehr $\ddot{U}$ berblick über das Leistungsverhalten und die Handlungssituation des Agenten erhalten kann, wird der Handlungsspielraum transparenter gemacht und einem opportunistischen Verhalten seitens des Agenten kann entgegengewirkt werden (vgl. Ebers/Gotsch, 1999).

\subsubsection{Gestaltungspotentiale}

Die Leistungserstellung zeichnet im Rahmen der Agenturtheorie von einer effizienten vertraglichen Gestaltung von Agenturbeziehungen abhängig. Ein ökonomischer Tausch, sei es jetzt ausgeführt innerhalb oder außerhalb eines Unternehmens, vollzieht sich auf der Basis einer Vereinbarung (implizit oder explizit) zwischen unvollständig informierten und nutzenmaximierenden Individuen mit widersprüchlichen Zielen (in diesem Punkt lässt sich eine gewisse Analogie zum „Koalitionsmodell der Organisation“ von Cyert/March, 1963 erkennen). Durch die einfache und präzise Theoriekonstruktion erleichtert die Agenturtheorie die For- 
mulierung von Hypothesen über Koordinationsprobleme, wodurch sie Eingang in eine Vielzahl von empirischen Studien gefunden hat (Ebers/Gotsch, 1999). Der Anwendungsbereich der Organisation, wo die Delegation von Aufgaben und Entscheidungsbefugnissen häufig auftritt und Austauschbeziehungen zwischen Manager und Unternehmer vorhanden sind, ist für die Agenturtheorie ein vielseitiger. Einem suboptimalen Verhalten von Managern muss entgegengewirkt werden (Fama/Jensen, 1983a, 1983b; Fama, 1980; Jensen/Meckling, 1976).

Um diesen organisationstheoretischen Mangel zu überwinden, wird die Disziplinierung von Managern auch oftmals durch die Tätigkeit eines Kontrollorgans in der Führungsspitze eines Unternehmens sicherzustellen versucht (in den USA durch ein Board of Directors oder in Deutschland und Österreich durch einen Aufsichtsrat), wobei die Einflussnahme auf diese spezifische Funktion der Kontrolle oftmals nicht in dem Ausmaß wahrgenommen wird, dass tatsächlich die Agenturprobleme wie "hidden action" oder "hidden information" überwunden werden.

Weitere Mechanismen, die das Leistungsverhalten von Managern auf die Zielvorstellungen des Prinzipals ausrichten helfen, sind beispielsweise Marktmechanismen, der Markt für Unternehmungskontrolle oder der Arbeitsmarkt für Manager, wobei sich Ausführungen zu diesen Themen bei Fama/Jensen (1983a, 1983b), Fama (1980), Picot/Michaelis (1984), Jensen/Meckling (1976), Frank Mayer (1990) finden.

Die Agenturtheorie vertritt eine ex-ante-Perspektive, wobei ein Prinzipal einen Vertrag in vollem Bewusstsein der Risiken abschließt, die die Vertragserfüllung durch den Agenten mit sich bringt. Probleme, die nach dem Vertragsabschluss auftreten können (vgl. Williamson, 1985), werden vernachlässigt, was die Realistik der Theorie jedoch schmälert. Auch die einseitige Betrachtung von Seiten des Prinzipals aus erscheint problematisch. Den Agenten betrachtet man im Lichte von opportunistischem Verhalten, das der Prinzipal jedoch nur selten, wenn überhaupt aufweist (Beiträge, die auch den Opportunismus des Prinzipals behan- 
deln, finden sich z.B. bei Taylor, 1978; Hart, 1983). Durch die Idee eines "MultiAgent-Modells" wird das agenturtheoretische Grundmodell relativiert und zugleich erweitert, wodurch die Agenturtheorie auf Situationen mit mehreren Agenten angewandt wird und wodurch Austauschbeziehungen, die sich in einem Unternehmen finden, weit realistischer widergespiegelt werden (Föhr, 1991; Arrow, 1985). Auch die Annahme der vorweg fixierten Zielvorstellungen der einzelnen Akteure (Levinthal, 1988) wird angezweifelt (March, 1988a). Denn bei Festlegung der Zielvorstellungen der Vertragspartner müssen die Phänomene wie "hidden information" und "bounded rationality" eine Berücksichtigung erfahren, sodass eine Ambiguität von Präferenzen gekennzeichnet werden kann.

Trotz des in sich geschlossenen Theoriekomplexes kam es auch zu kritischen Einwänden und konzeptuellen Erweiterungen hinsichtlich der vertragstheoretischen Ausrichtung zwischen den Parteien, die die Komplexität von Institutionen zu vernachlässigen scheint. So wird der Rolle von regulativen Dritten zu wenig Bedeutung beigemessen, was das Vorhandensein von staatlichen Regelungsmechanismen wie Gesetzen oder gerichtlicher Rechtsprechung außer Acht lässt (vgl. Ebers/Gotsch, 1999).

Ein Gestaltungsbeitrag der Agenturtheorie zur Entwicklung eines Wissensmanagement-Ansatzes erschließt sich in der empfohlenen Konzeptionalisierung der Arbeitsteilung und Spezialisierung sowie des Tausches und der Abstimmung in einem Unternehmen, die in dieser Theorie integrativ gezeichnet wird. Eine unproduktive Arbeitsteilung bzw. eine Fehlspezialisierung wird durch die Agenturkosten explizit berücksichtigt und manifestiert sich als Abweichung von einem "optimalen" Austauschszenario. Ein institutionelles Rahmengerüst zu designen, das die Agenturkosten minimiert, bedeutet zwangsläufig, einen wohlstandsmaximierenden Kompromiss zwischen einer möglichst produktiven Arbeitsteilung und Spezialisierung sowie möglichst "reibungslosen“ Tausch- und Abstimmungsvorgängen zu finden (vgl. Picot/Diet//Franck, 1997). 
Um die einem Unternehmen zugrunde liegenden Informationsasymmetrien im Rahmen eines Wissensmanagements zu überwinden, zeigen sich die institutionellen Arrangements (die im Rahmen des Kapitels „Entwurf eines Wissensmanagements" näher erläutert werden), die die Agenturtheorie entwickelt hat, um die zu befürchtenden Verhaltensprobleme jeweils unter Inkaufnahme der geringst möglichen Agenturkosten zu begrenzen, als viel versprechend. 


\subsection{Abschließende Betrachtung}

Die Theorie der Neuen Institutionenökonomie bietet einen komplexen und in sich geschlossenen theoretischen Unterbau für einen breiten Anwendungsbereich. Sie verfügt über klare Erklärungsansätze für Kernfragen im Bereich der Organisationsforschung, wie beispielsweise welche institutionellen Arrangements eine effiziente Faktorallokation bzw. eine effiziente Gestaltung und Anpassung der Leistungserstellung an sich ändernde Umweltgegebenheiten gewährleisten. Vertreter der Neuen Institutionenökonomie behaupten jedoch nicht, dass die einzelnen Theoriestränge vollständig ausgebaut sind und die endgültigen Antworten auf die optimale Wahl von institutionellen Arrangements gefunden wurden, jedoch gibt die Theorie der Neuen Institutionenökonomie ein breites Spektrum an Erklärungsfaktoren und identifiziert grundlegende Gestaltungsalternativen (Williamson, 1985, S. 390ff).

Das Feld der Neuen Institutionenökonomie wird alleine durch die drei Hauptströmungen sehr inhomogen, da sich diese untereinander in ihrer Theoriekonstruktion und in ihren Anwendungsmöglichkeiten unterscheiden. Charakteristisch für jede Theorie sind die jeweils zugrunde gelegten Verhaltensannahmen, die Wahl und die Beschreibung der untersuchten Institution, die Charakterisierung der Austauschsituation sowie die Bestimmung der Kosten und der Effizienz. Reduziert die Theorie der Verfügungsrechte das Handeln von Akteuren auf eine individuelle Nutzenmaximierung, so wird diese Verhaltensannahme im Rahmen der Transaktionskosten- und Agenturtheorie bereits um die Charakteristika "Opportunismus" und "beschränkte Rationalität" ergänzt. Zudem bezieht die Agenturtheorie die Risikobereitschaft und -aversion der Akteure prinzipiell in die Analyse ein. Die Verhaltensannahmen der beschränkten Rationalität und die nur selten erwähnte Annahme der Risikoneutralität wurden weithin akzeptiert, jedoch war die Perspektive des Opportunismus Gegenstand zahlreicher Kontroversen (Goshal/Morgan, 1996; Barney, 1990; Donaldson, 1990). Dieser Kritik wurde jedoch entgegengehalten, dass der opportunistischen Verhaltensannahme nicht ein negativ ausgerichtetes Menschenbild zugrunde liegt (vgl. Williamson, 1985). 
Die Wahl der Institution als Untersuchungsgegenstand erschließt sich im Rahmen der Theorie der Verfügungsrechte in der Verteilung und Gestaltung von Verfügungsrechten, wobei in der Agentur- und Transaktionskostentheorie die Verfügungsrechtsstrukturen nicht weiter thematisiert werden und die Institution des Vertrages selbst in den Mittelpunkt gerückt wird.

Die Austauschbeziehungen sind in allen drei Theorien von Interesse. Im Rahmen der Transaktionskostentheorie wird neben der Häufigkeit und Unsicherheit der Transaktion insbesondere die Problematik transaktionskostenspezifischer Investitionen betont. Die Agenturtheorie hingegen beschreibt die Austauschsituation mit Hinweisen auf die ungleiche Informationsverteilung, die Verteilung von Risiken und bestehenden Unsicherheiten. Einzig allein die Theorie der Verfügungsrechte sieht keine spezifische Charakterisierung der Austauschbeziehungen vor (vgl. Ebers/Gotsch, 1999).

Trotz einer umfassend geäußerten Kritik an der Theorie der Neuen Institutionenökonomie sollen ökonomische Theorien genau dort ihren Platz einnehmen, wo es Effizienzkriterien zu formulieren und Hinweise zur Vertrags- und Organisationsgestaltung zu geben gilt. Kritische Einwände (vgl. z.B. Putterman, 1984; Robins, 1987; Demsetz, 1967; Türk, 1997; Göhler/Kühn, 1999) können dabei als Quelle für die Verbesserung und Ausspezifizierung der Theorie dienen. Eine fundamentale Kritik äußern hingegen Vertreter einer soziologisch geprägten Disziplin, die sich beispielsweise gegen eine Ignoranz gegenüber institutionellen Einflüssen jenseits transaktionskostenrelevanter politischer und rechtlicher Regeln (Granovetter, 1985; Perrow, 1986), gegen ein von der Realität abgehobenes adhoc-Theoretisieren (Simon, 1991) sowie gegen die grundsätzlich unzulänglich definierten Verhaltensannahmen und die opportunistisch geprägte Handlungsorientierung (Barney, 1990; Ghoshal/Moran, 1996) aussprechen.

Die Konkurrenzbeziehung zwischen der Theorie der Neuen Institutionenökonomie und dem soziologisch geprägten Pendant, dem Neoinstitutionalismus (vgl. Granovetter, 1985; Meyer/Rowan, 1983; Scott, 1995a/b; DiMaggio/Powell 1991), 
dominiert gemeinhin die Diskussion über Institutionen. Weniger werden hier Unterschiede zwischen den Disziplinen ausgetragen, als auf divergierende organisations-wissenschaftliche Prämissen und analytische Schwerpunkte eingegangen wird. Die unterschiedliche Definierung eines Handlungs- und Rationalitätskonzepts, die bekannte Dichotomie zwischen dem "homo oeconomicus" und dem "homo sociologicus", Gegensätze wie Individualismus versus Kollektivismus oder Strukturtheorie versus Handlungstheorie rücken allesamt ins Lichte der Kontroverse (vgl. beispielsweise DiMaggio/Powell, 1991; Scott, 1995a; Göhler/Kühn, 1999).

Trotz der stark divergierenden Annahmen, Argumentationen sowie unterschiedlichen Analyseebenen der Neuen Institutionentheorien lassen sich eine Annäherung der Ansätze und ein transdisziplinärer Rekurs auf institutionelle Ansätze beobachten. So finden sich Bestrebungen einer Synthese des soziologischen Neo-Institutionalismus und der Theorie der Neuen Institutionenökonomie beispielsweise bei Tolbert/Zucker (1996), Roberts/Greenwood (1997), Göhler/Kühn (1999). Diese noch junge und wenig ausgereifte Entwicklungslinie wird positiv bewertet und die Verbindung zwischen den beiden Theorierichtungen könnte zu einer Bereicherung der ökonomischen Institutionentheorien führen (Edeling, 1999).

So lassen teilweise einzelne Vertreter der Neuen Institutionenökonomie erkennen, dass eine Annäherung an die Betrachtungsweise des soziologischen Institutionalismus stattfindet (vgl. bspw. North, 1998). Institutionen sollen nicht wie bisher nur auf den regulativen Aspekt beschränkt gedacht werden. Es wird anerkannt, dass wirtschaftliches Handeln und die Gestaltung von Organisationen auch von anderen Institutionen, z.B. gesellschaftlichen Normen, beeinflusst wird bzw. dass gesetzliche Regelungen legitim erscheinen, d.h. normativ verankert sein müssen, um wirksam werden zu können. Rationalität, Wahlhandlungen in Organisationen und die Suche nach effizienten organisationalen Lösungen müssen innerhalb einer Gesellschaft und einer historischen Epoche verstanden und erklärt werden (Walgenbach, 2002).Sarah Meisenberger - 978-3-631-75453-5 
Es wird über den Begriff der beschränkten Rationalität, wie er als Verhaltensannahme sämtlichen drei Teiltheorien der Neuen Institutionenökonomie zugrunde gelegt wird, hinausgegangen. Die Begrenzung der Rationalität liegt nicht nur im Individuum und dessen begrenzter Informationsverarbeitungskapazität begründet, sondern auch im historisch-gesellschaftlichen Kontext, in dem eine Entscheidung getroffen wird. Weil bestimmte strukturelle Elemente und bestimmte Formen organisationaler Gestaltung auch normativ und kognitiv institutionalisiert sind, werden von den Entscheidungsträgern in Organisationen weder alle denkbaren noch alle bekannten Alternativen in Betracht gezogen (Walgenbach, 2002).

Da von einer problemlosen Verbindung zwischen neoinstitutionalistischer Organisationstheorie und institutionenökonomischen Theorien nicht ausgegangen werden kann, da dafür die grundlegenden Annahmen in den beiden Theorien zu unterschiedlich sind und noch wesentliche Vorarbeiten zu leisten sind ( $\mathrm{vgl}$. Walgenbach, 2002), wird die Verschmelzung dieser beiden Theorien auch nicht für die vorliegende Arbeit übernommen. 


\section{Ein Entwurf eines Wissensmanagement-Ansatzes}

\section{Die einzige Gewähr für das wirkliche Wissen ist das Können.}

Paul Valéry

\section{1. Überblick}

„Vorgestern hatten wir Lean Management, gestern Total Quality Management, heute Process Reengineering und die Konzepte für morgen und übermorgen werden bereits entwickelt. All diese Projekte haben ihre Spuren aller Art in unserem Unternehmen hinterlassen. TQM-Handbücher, Prozessbeschreibungen, Informationssysteme [...]. Wenn ich alles anwenden würde, was ich nutzen müsste, würde ich nicht mehr zum Arbeiten kommen." (Manager eines Industriekonzerns zit. in Romhardt, (1998a), S. 231f.)

Im Zuge der Entwicklung von neuen Managementkonzepten treten immer auch Gegenpositionen auf. Dies ist eine Situation, die jedem Fortschritt inhärent ist. Das Streben nach Lösungen für eine fortwährende Effizienz- und Effektivitätssteigerung von Unternehmen ist seit jeher Gegenstand der Organisationsforschung (vgl. beispielsweise Penrose, 1959; Zand, 1969; Rickson, 1976; Zuboff, 1988). Lösungsvorschläge wurden immer wieder aufs Neue revidiert und verbessert, sodass gerade in Anwendung gebrachte Konzepte bereits nach kurzer Zeit wieder veraltet und unangemessen erschienen.

Wettbewerbsvorteile lassen sich vor allem durch bessere, schnellere und intelligentere Problemlösungen hinsichtlich der Produktion von Gütern oder des $\mathrm{Ge}$ bens von Dienstleistungen erlangen und verteidigen. Ein Wettbewerb der Problemlösungen beruht in erster Linie auf Wissens-vorsprüngen. In Wissensmanagement wird Hoffnung gesetzt, die "black box" Unternehmen besser auszuleuchten, um durch ein gutes Verständnis über interne Leistungsprozesse Wettbewerbsvorteile gegenüber den Mitbewerbern zu erreichen. 
„As a company focuses ever more on its own internal knowledge and senvice skills and those of its suppliers, it increasingly finds that managing shifts away from the overseeing and deployment of fiscal and physical assets and toward the management of human skills, knowledge bases, and intellect both within the company and in its suppliers. In fact, its raison d'être becomes the systematic coordination of knowledge and intellect throughout its (often highly disaggregated) network to meet customer needs" (Quinn, 1992, S. 72).

Im Zuge der Erstellung von Gütern sowie des Anbietens von Dienstleistungen werden jedoch innerhalb arbeitsteiliger Leistungsbeziehungen Koordinationssowie Motivationsprobleme auftreten (vgl. Milgrom/Roberts, 1992; Picot/Diet// Franck, 1997), worauf bereits in Kapitel 5.2 eingegangen wurde. Da der Überwindung des Organisationsproblems, worunter beide genannten Problemfelder subsumiert werden, eine Schlüsselrolle in einem Wissensmanagement zugesprochen wird, werden die Eckpunkte des Organisationsproblems nochmals wiederholt und leiten auch gleichzeitig die Ausführungen des im Folgenden entwickelten Wissensmanagement-Ansatzes ein.

Das Koordinationsproblem setzt sich mit dem Nichtwissen von Akteuren auseinander und es tritt auf, wenn Individuen die Rolle, die innen in einem Unternehmen zukommt, unbekannt ist. Dies kann aufgrund von zwei verschiedenen Szenarien auftreten, zwischen denen das Koordinationsproblem jedoch nicht differenziert. Einerseits können sich Individuen einem Entscheidungs- bzw. Wahlproblem gegenübersehen; dem Problem, dass der Akteur nicht weiß, was er tun soll. So kann das Individuum bspw. mehrere Alternativen aus einer Anzahl möglicher Handlungsoptionen zur Auswahl haben, jedoch nicht wissen, welche davon die richtige ist. Andererseits kann es sich einer Situation gegenübersehen, in der es nicht weiß, wie es das, was es für "richtig" hält, tun soll. Ein Motivationsproblem besteht dagegen in den Fällen, in denen Akteure die innen zugewiesenen Rollen gar nicht spielen wollen (vgl. Milgrom/Roberts, 1992). 
Im Zuge von Wissensmanagement sollte gewährleistet werden, dass die einzelnen Akteure eines Unternehmens klar darüber Bescheid wissen, welche Rolle innen zugesprochen wird. Zum anderen sollten Bestrebungen in die Richtung unternommen werden, dass die Mitarbeiter eines Unternehmens diejenigen Fähigkeiten erlangen, die sie benötigen, um die innen zugedachten Rollen ausfüllen zu können. Im ersten Fall handelt es sich um die Koordination einer auf eine gegebene Menge von Akteuren aufgeteilte Menge an Aktivitäten, während es im zweiten Fall darum geht, auf welche Weise überhaupt die Aufteilung der für die Leistungserstellung erforderlichen Fähigkeiten und Kompetenzen auf die einzelnen Akteure erfolgt. Der beste Koordinationsmechanismus ist jedoch nutzlos, wenn Akteure die ihnen zugewiesenen Rollen nicht ausführen können oder wollen. Ebenso ineffizient sind hoch motivierte gut ausgebildete Akteure, die nicht wissen, was sie tun sollen. Daher wird es im Zuge einer Entwicklung eines Wissensmanagement-Ansatzes als unumgänglich angesehen, simultan die Aspekte Koordination sowie Motivation zu berücksichtigen.

Im Rahmen traditioneller Managementkonzepte wurden oftmals Individuen als zentrales Element von Organisationen betrachtet. Führt man diese Überlegungen weiter, so stößt man sehr schnell auf die Unzulänglichkeit dieser Annahme. Es ist kritisch und problematisch davon auszugehen, dass der Mensch in seiner Gesamtheit im Mittelpunkt der Analyse stehen kann. Wenn letztere innerpsychische Unendlichkeiten, lebensbiographische Verläufe, unbewusste Anteile, private Beziehungen, Glaubensüberzeugungen etc. beinhaltet, dann stoßen klassische Managementüberlegungen schnell auf kompetenzbezogene und auch ethische Grenzen (Kasper/Mayrhofer/Meyer, 1999).

Das Menschenbild wird in der Theorie der Neuen Institutionenökonomie bewusst zur Verringerung der menschlichen Komplexität eingeschränkt gezeichnet, um organisationstheoretische Fragestellungen beantworten zu können. Es zeigt sich von Interesse, wie Individuen, die als Quelle von Wissen ausgemacht wurden, in einer für das jeweilige Unternehmen effizienten und effektiven Weise in der Leis- 
tungserstellung integriert werden können, damit Unternehmen einen Wissensvorsprung gegenüber ihren Wettbewerbern erlangen können.

Institutionen werden gebildet, um das Verhalten von Individuen in eine bestimmte Richtung zu lenken und Ordnung in die alltäglichen Tätigkeiten von Organisationen zu bringen. Sie ermöglichen die Überwindung des Organisationsproblems durch ihren regulativen und gleichzeitig gestalterischen Charakter. In der vorliegenden Arbeit werden Organisationsstrukturen als Institutionen verstanden (Picot/Diet//Franck, 1997, S. 31), die den Handlungsspielraum der Unternehmensmitglieder eingrenzen und ihr Verhalten zielgerichtet steuern, um auf diese Weise Koordination und Motivation zu gewährleisten.

Bevor jedoch näher darauf eingegangen wird, wie Organisationsstrukturen geformt werden sollen, um ein Koordinations- und Motivationsproblem mithilfe eines Wissensmanagements zu überwinden, soll die diesem WissensmanagementAnsatz zugrunde gelegte Wissenskonzeption näher erläutert werden. Eine unspezifische Wissenskonzeption kann keine sinnvolle Grundlage für ein erfolgreiches Wissensmanagement bilden (Schreyögg/Geiger, 2003).

Organisationsstrukturen werden auf eine solche Weise gebildet, dass sie formale Kommunikationsstrukturen widerspiegeln und auf ein definiertes Unternehmensziel ausgerichtet sind, um einen Unternehmenszweck zu erfüllen. Das Individuum spielt dabei eine tragende Rolle und so kommt es auch zu einer Analyse dessen, welche zusätzlichen Strukturen integriert werden müssen, die bspw. Anreiz-, Steuerungs- und Kontrollmechanismen beinhalten, um den Mitarbeitern eines Unternehmens die Möglichkeit zu geben, das Unternehmensziel so weit als ihr eigenes anzunehmen, dass sie motiviert sind, dieses auch zu erreichen. Auf Weiterbildungsmaßnahmen und Schulungen von Mitarbeitern wird ein besonderes Augenmerk gelegt, da als ein Haupterfolgsfaktor für ein Wissensmanagement das Verfeinern von Wissen herauskristallisiert wird. Schlussendlich ordnet sich auch die Informations- und Kommunikationstechnologie ein, die ihren Teil dazu beiträgt, als Garant für ein erfolgreiches Wissensmanagement zu fungieren, 


\subsection{Handlungsrelevantes Wissen}

Es ist nicht genug zu wissen, man muss auch anwenden; es ist nicht genug zu wollen, man muss auch tun. Johann Wolfgang von Goethe

Legt man die Theorie der Neuen Institutionenökonomie den in dieser Arbeit durchgeführten Ausführungen zugrunde, positioniert man sich klar in einem funktionalistischen Paradigma. Dadurch verlangt auch die Auseinandersetzung mit Wissen eine an der funktionalistischen Logik orientierte Ausrichtung. "It is a perspective which is highly pragmatic in orientation, concerned to understand society in a way which generates knowledge which can be put in use. It is often problemorientated in approach." (Burrell/Morgan, 1979, S. 26) Es wurde bereits konstatiert, dass diese Arbeit auf die Wissenskonzeption von Polanyi (1962) rekurriert. Nähere Ausführungen zu dieser Wissenssystematisierung finden sich ebenfalls im Rahmen der institutionenökonomischen Theorien. Wissen wird einerseits in "communicable knowledge" und andererseits in "tacit knowledge" getrennt. Ersteres beinhaltet Wissen, das von einer Person zu einer anderen transferiert werden kann, und letzteres wird durch Praxis und Übung angenommen und kann nur teilweise, wenn überhaupt, kommuniziert werden (North, 1990).

"Communicable knowledge" stellt das Wissen dar, das in artikulierter, transferierbarer und archivierbarer Form vorliegt und nach bestimmten Konstruktionsregeln reproduzierbar ist (als Synonyme werden oftmals die Begriffe "explizites Wissen“ oder "disembodied knowledge" verwendet). "Tacit knowledge" hingegen ist viel schwieriger zu fassen, auch dadurch, weil sich die Literatur bei der Definierung dieses Begriffs in keinster Weise einig ist. Ursprünglich konstatierte Polanyi (1962) „implicit knowledge" (eben auch als "tacit knowledge" bekannt) als „embodied" oder "personal", was bedeutet, dass es vom Menschen abhängig und nicht separierbar ist. Es wird auf den Sachverhalt rekurriert, dass zahlreiche Aspekte des Verstehens und Könnens des Individuums nicht in Worte gefasst sind bzw. nicht gefasst werden können. Dieses Wissen liegt dem Handeln unbewusst 
zugrunde und ist an die Erfahrungsträger (Personen) gebunden. Begriffe wie "Erfahrungsschatz", "Intuition" oder "Kreativität" kommen nahe an eine exakte Beschreibung dieses Wissenstyps heran. Im täglichen Handeln und Arbeiten kann man sich auf dieses Wissen verlassen, ohne dass es unbedingt bewusst sein muss (Schreyögg/Geiger, 2003).

Wissen wird nicht einseitig als etwas Statisches angesehen, sondern dem dynamischen Aspekt von Wissen wird eine gleichbedeutende Beachtung geschenkt. So kommt es zu der Betrachtung, dass Wissen durch das Handeln selbst und die dadurch erlangte Sicherheit gewonnen und gefestigt wird. Zieht eine Person die für sich als relevant erachteten Informationen in Betracht und übt sich in deren Anwendung, so wird sie auf eine Kenntnis über die behandelten Sachverhalte zurückgreifen können und ein dementsprechendes Bewusstsein darüber erlangen, wie Tatbestände und Vorgänge vor sich gehen und sich darstellen. Wissen ist somit "volles Verstehen von einer Sache und die Gewissheit, anwenden zu können" (Duden, 2003, Definition "Wissen").

Die für ein Individuum als relevant erachteten Informationen schneiden einen weiteren Bereich von Wissen an, nämlich, dass Wissen vom jeweiligen Kontext abhängig zeichnet. Das Umfeld, in dem Individuen eingebettet sind, beeinflusst deren Wahrnehmung. Das Wissen, das Individuen von der Welt haben, lässt sie immer die Welt mit ihren eigenen Augen betrachten und somit beeinflusst dies die Art und Weise, wie sie etwas rationalisieren, erklären und rechtfertigen. "Knowledge is deeply rooted in the context in which institutions are embedded. The way knowledge develops shapes the perceptions of the world around and in turn those perceptions shape the search for knowledge" (North, 1990, S. 76).

Individuen sehen sich verschiedensten Tätigkeiten und Problemstellungen gegenüber. Durch ein Set an Institutionen werden sie in ihrem Handeln unterstützt, um nicht zu viel Zeit und Mühen aufwenden zu müssen, um Entscheidungen zu treffen, Probleme zu lösen und Handlungen durchzuführen. So können sie auf ein stabiles Gleichgewicht und Routinewissen zurückgreifen worin sie ihre Auf- 
gabenstellungen als regulär und sich wiederholend einordnen können. Institutionalisiertes Wissen legitimiert Handlungen einer gegebenen Situation und gewährleistet eine verlässliche Qualität des Outputs (Stein, 1997). Zusätzlich stabilisiert die Integration von Wissen in Routinen ein Unternehmen von innen her (Stein, 1997), was wiederum dazu führt, dass das Unternehmen in seiner Beziehung zu seinem Umfeld eine gewisse Stabilität erreichen kann. Indem Wissen und die soziale Wirklichkeit als selbstverständlich vorausgesetzt werden, internalisieren Individuen Wissen durch den Prozess der Sozialisation und durch learning by doing (North, 1990). Institutionen (seien es informelle oder formale) fungieren als Repräsentanten von institutionalisiertem Wissen, das nicht weiter hinterfragt, sondern benutzt und angewandt wird.

Ansätze, die sich von Polanyis Verständnis von implizitem und explizitem Wissen ableiten, werden unter den wissensbasierten Ansätzen zusammengefasst. Der Fokus liegt hier auf der Betrachtung von Wissensstrukturen, -prozessen, dem organisationalem Gedächtnis und der Lernfähigkeit (beispielsweise zählen hierzu die Ansätze von Pautzke, 1989; Nonaka/Takeuchi, 1995). Explizites Wissen kann mit Hilfe von Sprache artikuliert und transferiert werden, was sich in detaillierten Prozessbeschreibungen, Organigrammen oder Patenten ausdrückt. Implizites Wissen hingegen basiert auf individuellen Erfahrungen und kann nicht durch sprachliche Mittel artikuliert und transferiert werden.

Implizites Wissen wird als eine Art des Wissens akzeptiert, konträr zu der Position von Schreyögg/Geiger (2003, S. 15), die konstatieren:, "dass implizites Wissen im eigentlichen Sinne kein Wissen sein kann. Weder handelt es sich dabei um Aussagen ("nicht-verbalisierbar"), noch ist der gemeinte Sachverhalt begründbar oder gar einem Prüfverfahren zu unterwerfen. Damit ist beim impliziten Wissen ein Qualifizierungsverfahren nicht anwendbar und es eignet sich damit nicht für ein systematisches Wissensmanagement, weil es sich der Selektionsaufgabe grundsätzlich entzieht. Es scheint wichtig darauf hinzuweisen, dass mit dieser Feststellung nicht die Bedeutung impliziten "Wissens" bestritten wird. Intuition, Geschicklichkeit, Teamfähigkeit usw. sind ohne Zweifel wichtige Erfolgsfak- 
toren. [...] [wir] plädieren jedoch dafür, dies nicht als Wissen zu begreifen, angemessener wäre vielmehr von Könnerschaft zu sprechen."

Durch implizites Wissen werden spezifische individuelle geistige sowie körperliche Fertigkeiten hervorgehoben, denen in Organisationen eine spezielle Beachtung geschenkt werden sollte. Im Rahmen von wissensbasierten Ansätzen wird die zentrale Frage der Mobilisierung von implizitem Wissen in Organisationen und dessen Transfer auf die Organisationsebene in den Mittelpunkt gerückt. Lernen bzw. Wissensentwicklung wird als interaktiver Prozess zwischen diesen beiden Wissenstypen auf verschiedenen Ebenen der Organisation beschrieben (Lang/Winkler/Weik, 2001). Der Hauptschwerpunkt der Forschungsarbeiten im Bereich wissensbasierter Ansätze wird auf den Prozess der Wissensanschaffung und -generierung gelegt (Grant, 1996).

In der vorliegenden Arbeit soll dieser Fokus nicht übernommen werden. Das Unternehmen wird als eine wissensintegrative Einheit verstanden (,knowledgeintegrating institution", dieser Ausdruck wird von Grant, 1996 übernommen). Dies bedeutet, "that the primary role of firms is in the application of existing knowledge to the production of goods and services" (Grant, 1996, S. 112). Es zeigt sich von Interesse, sich näher mit explizitem Wissen, das in Organisationsstrukturen institutionalisiert wurde, auseinander zusetzen. Denn Institutionen, die sich in Organisationsstrukturen manifestieren, setzen den Handlungsspielraum von Individuen fest. Es kommt zu einer klaren Definierung, wie eine interne Leistungserstellung erfolgen soll. Dieses Wissen ist auf institutionalisierter Ebene vorhanden. Mithilfe einer klaren Arbeitsteilung und Spezialisierung innerhalb eines Unternehmens eignen sich Akteure die innen zugesprochenen Rollen an und internalisieren das für die von innen erwartete Leistungserstellung relevante Wissen. Sie bereichern dieses durch das innen eigene implizite Wissen.

Welches Wissen sich nun für ein Unternehmen als handlungsleitend darstellt, wird durch das Wissen bestimmt, das direkt zur Erfüllung der definierten Unternehmensziele und des bestimmten Unternehmenszwecks beiträgt Dies kann in 
der Unternehmerpraxis stark variieren und wird situations-abhängig über einen komparativen Vorteil entscheiden (Drucker 1992, Prusak, 1997; Hamel/Prahalad, 1994; Probst/Büchel/Raub, 1998).

Wird Wissen in der Form betrachtet, dass es in einer wechselseitigen Beziehung zu dem Handeln von ökonomischen Akteuren steht, kann gewährleistet werden, dass Wissen keinen Selbstzweck erhält. Wissen ermöglicht somit die Handlungen von Individuen, erzwingt sie jedoch nicht. Wissen stellt Optionen bereit und legt die Handlungs- und Gestaltungsspielräume der Beteiligten fest. "The opportunity set confronting an individual or a firm is a function of the individual's knowledge" (Jensen/Meckling, 1992, S. 253).

Ein Wissensmanagement-Ansatz, der eine solche Wissenskonzeption zum Mittelpunkt seiner Ausführungen macht, lenkt den Fokus auf Organisationsstrukturen. Es gilt, die Unternehmensziele mithilfe der in einem Unternehmen vorhandenen institutionellen Handlungsabläufe über eine Koordination und Motivation von Mitarbeitern zu erreichen. Auf welche Weise dies erfolgen kann, soll in den folgenden Kapiteln näher erläutert werden. 


\subsection{Organisation - Ordnung und Struktur verleihen}

[Es gibt] zwei Dinge, auf denen das Wohlgelingen in allen Verhältnissen beruht. Das eine ist, dass Zweck und Ziel der Tätigkeit richtig bestimmt sind. Das andere aber besteht darin, die zu diesem Endziel führenden Handlungen zu finden. Aristoteles

Organisationsstrukturen bilden das Fundament einer Organisation. Unternehmen, die durch eine gute Organisation die Komplexität der internen Gegebenheiten sowie der externen Umwelt reduzieren können, stützen sich bei der Erfüllung ihrer Aufgabenstellungen und Zielsetzungen auf einen klaren und formalen Aufbau ihrer Tätigkeiten. Formale Regelsysteme gewährleisten, dass jedem Mitarbeiter Verantwortlichkeiten, Pflichten und Rechte übertragen werden und dass dieser davon Kenntnis hat, welche Arbeit er für die Erreichung von Unternehmenszielen zu erledigen hat. Diese Formalisierung kann sich auf einer Ebene manifestieren, wo sie als institutionalisiert gilt und die Funktion von Institutionen (wie Reduktion von Unsicherheiten, Festsetzen von Spielregeln etc.) erfüllt. So können Mitarbeiter auf ein Routinewissen zurückgreifen, worin sie ihre Aufgabenstellungen als regulär und sich wiederholend einordnen und erledigen können, ohne ein zuviel an Reflexion durchführen zu müssen.

Effizient und effektiv zu produzieren und am Markt wettbewerbsfähig zu sein, impliziert, dass Individuen effizient und effektiv gemanagt werden müssen, was ferner impliziert, dass Wissen effizient und effektiv gemanagt werden muss. Dies kann nur dann erfolgreich durchgeführt werden, wenn man Organisationen versteht (Kakabadse/Kouzmin/Kakabadse, 2001). Die Organisationsform, die in einer Austauschbeziehung entsteht, hängt von der Effizienz dieser Form ab, damit eine notwendige Transaktion abgeschlossen werden kann (Coase, 1937). Interessant sei es zu untersuchen, wie sich Individuen arrangieren und koordinieren, um ihre Arbeiten erledigt zu bekommen. Organisatorische Strukturen sollten die 
Arbeitsbeziehungen innerhalb eines Unternehmens so gut wie möglich widerspiegeln, um Verantwortungsbereiche klar voneinander abgrenzen und bestimmen zu können. Dabei ist jedoch zu beachten, dass neben formalen Beziehungen, die leichter zu managen sind, auch informelle Netzwerke bestehen, die sich nur kaum, wenn überhaupt, einer äußeren Steuerung als zugänglich gestalten. Sie inkludieren die individuellen Wertevorstellungen, Normen, Erfahrungen und Ideen, die durch Interaktion mit anderen Mitarbeitern ausgetauscht werden. Eine Organisation besteht jedoch nicht aus einem formalen Regelsystem und dieser Wertevorstellungen etc., sondern beide Ordnungsstrukturen ergänzen sich zu einem Ganzen.

Die organisatorische Gestaltung eines Unternehmens stellt einen Einflussfaktor und eine Rahmenbedingung für Wissensmanagement dar. Traditionelle Organisationsstrukturen genügen meist nicht den Forderungen nach Flexibilität, Kooperation, Kommunikations- und Innovationsorientierung, die mit einer Wissensorientierung verbunden sind (vgl. beispielsweise Hedlund, 1994). So legen einige Konzepte ihr Augenmerk auf den gestalterischen Aspekt der Organisation, um Wissensprozesse steuerbar zu machen ( $\mathrm{vgl}$. beispielsweise Davenport/Prusak, 1998; Nonaka/Takeuchi, 1995, 2000; North, 2002; Probst et al., 1997; Rehäuser/Krcmar, 1996) und es wurden in jüngerer Zeit unterschiedlichste Organisationsmodelle entwickelt, wie bspw. die "Adhokratie", die "unendliche flache Organisation", „Spinnennetze", die "umgekehrte Pyramide", die "Hypertextorganisation", die "N-Form Corporation" etc., um die negativ assoziierte Formalisierung, Spezialisierung, Zentralisierung und Standardisierung rationaler Organisationsmodelle zu überwinden, da sie als zu schwerfällig für dynamische Umwelten gelten. Unternehmen sollen über flexiblere, dynamischere, anpassungsfähigere und partizipative Strukturen verfügen und Mitarbeiter in das Unternehmensgeschehen einbinden. Diese Modelle haben unter anderem gemeinsam, dass sie Wissen als einen der größten Unternehmensvorteile sehen (Nonaka/Takeuchi, 1999).

Wissen ist zwar in Fachabteilungen, Projekten und Geschäftsabteilungen gebunden, jedoch meist nicht in systematischer Weise aufbereitet, wodurch North 
(2002) die Schwierigkeiten eines Unternehmens bei einer wissensorientierten Unternehmensführung vor allem darin begründet sieht, dass eine Wissensorganisation fehlt, die Wissen mit einer über die kurzfristigen Geschäftsbedürfnisse hinausgehenden Perspektive systematisch nutzt und generiert. Hierbei müssen vor allem Wissensbarrieren überwunden werden, die nicht nur in hierarchischen Organisationen, sondern auch in Projekt- oder Prozessorganisationen zu finden sind. Sind es beispielsweise Verständigungsprobleme im ersten Fall, so verfügen Projekte selten über eine ausreichende Kontinuität und sind nur bedingt in der Lage, Erfahrungen systematisch aufzuarbeiten und über Projektgenerationen nutzbar zu machen. Im letzten Fall gelingt ein Wissenstransfer zwar entlang einer Prozesskette, jedoch nicht zwischen Prozessen. Der Kritik an Prozessorganisationen konträr gegenüber stehen die Ausführungen von Probst et al. (beispielsweise 1997) und Rehäuser/Krcmar (beispielsweise 1996). Sie schlagen sehr wohl eine Prozessorientierung ein und im Zuge von Wissensprozessen erschlieBen sie den organisationalen Wissensbestand bzw. streben dadurch an, diesen dem Unternehmen zugänglich zu machen. Vor allem Probst et al. (1997, S. 350) halten fest: „Die ideale Organisationsstruktur für Wissensmanagement existiert jedoch nicht. "Mittels Strukturen und Systemen gilt es lediglich, einen Kompromiss zwischen einander widersprechenden Ziel-vorstellungen zu schaffen.

North (2002) beklagt weiters an dieser Art von Organisation das Fehlen jeglicher Fähigkeit zur Infragestellung des Handelns und zur Erneuerung und er setzt sich detailliert mit den verschiedensten Organisationsformen und ihrer Kompatibilität mit Wissensmanagement (beispielsweise die Analyse einer unendlich flachen Organisation, einer invertierten Organisation, von Organisationen in der Form einer Sternexplosion oder eines Spinnennetzes) auseinander und entwickelt einen eigenständigen Vorschlag der strukturellen Gestaltung von Organisationsformen (vgl. North, 2002).

Geschäftsprozesse, die bereits im Zuge von Business-Process-Reengineering (BPR)-Maßnahmen im Mittelpunkt der Betrachtung gestanden sind, als es signifikante Zeit- und Kosteneinsparungen sowie Qualitätsverbesserungen seitens von 
Unternehmen zu erzielen galt, dokumentieren Wissen, was als Basis für eine Wissensanalyse dienen kann (vgl. Roehl, 2002). Es wird eine ganzheitliche Optimierung funktionsübergreifender Abläufe angestrebt, um Verbesserungen entlang der Wertschöpfungskette zu erreichen, indem bereichsorientierte Schnittstellen überbrückt bzw. koordiniert werden (vgl. Thiesse, 2001). Bestrebungen, ein Wissensmanagement im Unternehmen zu integrieren und dabei die bereits vorhandenen formalen Unternehmensprozesse zu nutzen, sind weithin als Business-Knowledge-Management bekannt geworden und verstehen sich vorwiegend als Ansatz zur Organisationsgestaltung. Ein prozessorientiertes Wissensmanagement greift jedoch durch seine Grundstruktur und durch grundsätzliche Aufgaben weiter als es Business-Process-Reengineering-Maßnahmen je getan haben. Ist vormals lediglich die Aufmerksamkeit auf standardisierte und repetitive Prozesse gelegt worden, so weisen wissensintensive Prozesse oftmals eine groBe Unstrukturiertheit und geringe Formalisierung auf, deren Ablauf nicht exakt modellierbar ist, sich im Zeitverlauf ändert und hochgradig von den ausführenden Personen abhängig ist (vgl. Davenport/Prusak, 1996). Somit müssen BPR-Bestrebungen erweitert werden und erfordern zusätzlich zur Betrachtung von Funktionen, Daten, Organisation und Kontrolffluss auch die explizite Berücksichtigung des notwendigen Wissens (sei es des expliziten oder aber auch des impliziten) (vgl. Scheer, 1998).

Einen Handlungsrahmen mit den Aufgaben des Wissensmanagements bieten die drei Ebenen des Wissensmanagements definiert durch Rehäuser/Krcmar (1996). Sie können erfüllt werden, wenn eine dafür geeignete Organisationsform vorhanden ist, wobei hierbei eine zielgerichtete Strukturierung von Personen, Sachmitteln und Informationen in Form von Interaktionssystemen verfolgt wird. Es reicht nicht aus, einzelne Abteilungen mit der Aufgabe des Wissensmanagements zu betrauen, sondern diese Aufgabe muss unternehmensübergreifend bestehen. Vor allem innovationsorientierte Strukturmodelle, die eine leistungsfähige Kommunikationsstruktur aufweisen, sind in der Lage, die geforderte Rahmenstruktur zu bieten. Diese Modelle inkludieren das Modell sich überlappender Gruppen, 
das Netzwerkmodell sowie die Hypertextorganisation (siehe dazu detailliert Rehäuser/Krcmar, 1996).

Hier soll lediglich die Hypertextorganisation eine gesonderte Erwähnung erfahren, da sie auf Nonaka/Takeuchi (1995) zurückgeht, deren WissensmanagementKonzept bereits dargestellt wurde (vgl. Nonaka/Takeuchi, 1995; 2000). Auf diese Organisationsform wird oftmals rekurriert (vgl. beispielsweise Rehäuser/Krcmar, 1996; Neumann, 2000; North, 2002; Probst et al., 1997). Die Hypertextorganisation stellt den Versuch einer Kombination von nichthierarchischen, selbstorganisierenden Strukturen und traditionellen hierarchischen Strukturen dar, wobei diese erfolgreiche Kombination als ein Schlüsselpunkt für die Wissensschaffung eines Unternehmens angesehen wird. Bereits der Begriff "Hypertext" ist bewusst gewählt worden, da er ausdrücken soll, dass sich eine Organisation aus miteinander verbundenen Schichten bzw. Kontexten zusammensetzt, nämlich Geschäftssystem, Projektteam und Wissensbasis (eine grafische Darstellung findet sich bei Nonaka/Takeuchi, 1997, S. 191). Erstere Schicht beinhaltet die Routinetätigkeiten eines Unternehmens und auf Grund der hierfür geeigneten bürokratischen Organisationsform wird sie in Form einer Pyramide dargestellt. Parallel dazu werden in einem Unternehmen auch Projekte (ausgerichtet auf eine Unternehmensvision) durchgeführt (beispielsweise für die Entwicklung von Neuprodukten), wobei Mitarbeiter der verschiedensten Bereiche aus dem Geschäftssystem in einem Projekt zusammenarbeiten. Das dort neu entstandene Wissen wird in einer darüber liegenden Schicht, der Wissensbasis, neu klassifiziert und beispielsweise in der Unternehmenskultur verankert. Diese Wissensbasis ist organisatorisch nicht eingegliedert. Die Koexistenz von drei völlig verschiedenen Schichten innerhalb einer Organisation kann als das Besondere dieser Organisationsform angesehen werden. Die organisatorische Fähigkeit der Wissenserzeugung wird dabei durch die Möglichkeit determiniert, zwischen den verschiedenen Wissenskontexten schnell und flexibel hin und her zu wechseln und damit einen dynamischen Kreislauf der Wissenserzeugung zu bilden (vgl. Nonaka/Takeuchi, 1995). 
Eine weitere Organisationsform, die eine besondere Bedeutung für eine wissensorientierte Unternehmensführung aufweist, wurde von Hedlund (1994) konzipiert und als N-Form-Organisation bekannt. Sieben Charakteristika zeichnen diese neue Form aus. Einerseits wird die Komplementarität von Ressourcen hervorgehoben, wobei das in einem Unternehmen vorhandene Wissen zur Generierung von Geschäftsfeldern kombiniert werden soll, bevor es geteilt wird, und Synergieverluste kompensiert werden müssen. Ein konstanter Pool an Mitarbeitern gewährleistet, dass gewisse Wertevorstellungen in einem Unternehmen etabliert sind, jedoch müssen diese eine Bereicherung durch das Wissen von neuen Mitarbeitern erfahren. So kommt es zwar dadurch zu Prozessen der Enkulturation, jedoch ermöglichen diese neuen Sichtweisen und die Fokussierung auf gemeinsame Interessen erst eine kollektive Wirklichkeitskonstruktion, mit der Wissensprozesse stattfinden können. Rekurrierend auf Nonaka/Takeuchi (1995) kommt es auch bei Hedlund (1994) zu einer Betonung der Bedeutung eines mittleren Managements. Es ist dafür zuständig, die von der Geschäftsführung bzw. dem oberen Management vorgegebene Unternehmensvision derartig in Konzepten zu konkretisieren, dass sie mit Hilfe der Mitarbeiter durchgesetzt werden kann. Dem oberen Management wird hierbei eine eigene Integrationsfunktion zugesprochen, um die Zielrichtung und Konsistenz von Prozessen der Wissensentwicklung sicherzustellen. Die Art und Weise, wie Mitarbeiter ihre Arbeiten erledigen sollten, kann durch sog. „best practices" ausgetauscht werden, wobei die Kommunikation vor allem vertikal über die Grenzen verschiedener Projekte und Geschäftseinheiten hinweg durchgeführt werden sollte. Heterarchische Unternehmensstrukturen können hierbei den Wissensaufbau und Wissenstransfer fördern.

Konzepte, die ihr Augenmerk unter anderem auf die Konzeptionalisierung von Organisationsstrukturen legen, versprechen sich dadurch, die einem Management inhärenten Planungs- und Steuerungsfunktionen erfüllen zu können und Mitarbeiter mitsamt ihrem Wissen sowie organisationale Wissensbestände bestmöglich für das Unternehmen einzusetzen. Dass im Zuge dessen verschiedenste Organisationsstrukturen entwickelt wurden sollte dieser kurze Abriss gezeigt 
haben. Dennoch bieten sie keine ausreichenden Lösungsvorschläge, um das Organisationsproblem zu überwinden.

Das theoretische Fundament, das hier die Theorie der Neuen Institutionenökonomie bildet, bietet einen Lösungsansatz, um Organisationsstrukturen auf ein Wissensmanagement hin zu erweitern und mithilfe von Koordinations- und Motivationsmechanismen, das Organisationsproblem einzudämmen. Im Zuge der Organisation eines Unternehmens sind Aufgabenverteilung, Verteilung von Weisungsrechten, Verteilung von Entscheidungsrechten und Programmierung die wesentlichen klassischen Gestaltungsvariablen, die jedoch durch die Strukturvariablen der Bereiche "Information" und "Kommunikation" ergänzt werden müssen (vgl. Picot/Dietl/Franck, 1999). Diese stellen eine sehr wichtige und notwendige Bedingung für die praktische Ausfüllung einer Organisationsstruktur dar (weitere Ausführungen hierzu finden sich beispielsweise bei Picot/Reichwald/Wiegand, 1998).

Im Rahmen eines funktionalistisch orientierten Organisationsverständnisses stellt die Organisation ein Ordnungsmuster zur Komplexitätsbewältigung dar. Dabei wird auf die Ausführungen rekurriert, die ein instrumentelles Organisationsverständnis zugrunde legen und Organisationen als ein System von Regelungen verstehen, um mehrere Aufgabenträger und ihre Arbeitsleistungen auf eine zu lösende Hauptaufgabe auszurichten, und Aufbau- und Ablaufstruktur der Organisation im Mittelpunkt der Betrachtungen stellen (vgl. Neumann, 2000, S. 112113).

Die formale Darstellung des Arbeitsgeschehens wird hierarchisch ausgerichtet, sodass das Beziehungsgeflecht eines Unternehmens abgebildet wird. Dem liegt ein Zielpluralismus, der materielle sowie immaterielle Zielvorstellungen mit einschließt, zugrunde, den es zu erreichen gilt (North, 1990). Unternehmen werden als Netzwerk relationaler Verträge zwischen Einzelpersonen zum Zwecke effizienter Organisation der Produktion verstanden (Williamson, 1989). Diese Verträge beruhen weitgehend auf freiwillig abgeschlossenen, durch die Rechtsord- 
nung geschützten langfristigen Verträgen, die in einem institutionellen Rahmen eingebettet sind. Die aus den verschiedensten Arbeitsverträgen resultierende Firmenhierarchie wird als effizientes Resultat der Minimierung von Transaktionskosten begriffen, was das Entstehen bzw. die Existenz von Firmen logisch begründet (Williamson, 1975).

Der Austausch von Waren und Dienstleistungen wird jedoch nicht ausschließlich mittels expliziter Vereinbarungen geregelt, sondern es bestehen in einem Unternehmen immer auch implizite Verträge. Sie formieren sich vor allem durch die in einem Unternehmen vorhandenen Verhaltensnormen und beeinflussen in einem großen Ausmaß das Unternehmensgeschehen. Die Beeinflussung des Verhaltens von Mitarbeitern durch ein formales Regelsystem stößt an seine Grenzen und die erfolgreiche Ausrichtung der Mitarbeiter auf ein Unternehmensganzes zeichnet stark von informellen Netzwerken abhängig. Diese manifestieren sich ebenfalls in den organisationalen Strukturen, da das Einhalten der Regelwerke im Interesse der Beteiligten liegt. Eine Fundierung dieser Strukturen auf der formalen Ebene wird jedoch als nicht notwendig erachtet. Unabhängig davon, um welche Verträge es sich nun handelt, fungieren diese einerseits als Beschränkungen und andererseits als Chancen. „Institutional constraints include both what individuals are prohibited from doing and, sometimes, under what conditions some individuals are permitted to undertake certain activities. As defined here, they therefore are the framework within which human interaction takes place" (North, 1990, S. 4). Hiermit lässt sich erkennen, dass dieser Arbeit ein weiter Vertragsbegriff zugrunde gelegt wird (Williamson, 1985). Formale Regelungen liegen informellen zugrunde (North, 1990). Auch wenn erstere lediglich selten die offensichtliche und unmittelbare Quelle für Entscheidungen im alltäglichen Leben sind (North, 1990), so stellen sie sich für ein Unternehmen als steuerbar dar, was unter anderem die Auseinandersetzung mit den formalen Organisationsstrukturen im Rahmen dieser Arbeit begründet. Sie gelten aufgrund ihrer Steuer- und Planbarkeit als Ausgangspunkt für ein Wissensmanagement. Implizites Wissen und das Wechselspiel zwischen explizitem und implizitem Wissen werden nach Erörterung der formalen Strukturen eines Unternehmens als weitere 
Einflussfaktoren im Rahmen eines Wissensmanagements betrachtet und Gegenstand näherer Erläuterungen sein.

Um eine Organisationsstruktur zu etablieren, die Wissensmanagement ermöglicht und das Organisationsproblem (Koordinations- und Motivationsproblem) überwinden hilft, werden etablierte Management-Konzepte Eingang in die weiteren Ausführungen finden. Dadurch wird gewährleistet, dass ein relativ junger Ansatz, wie Wissensmanagement, Altbewährtes (wie bspw. Forschungsbeiträge auf den Gebieten Organisationales Lernen, Total Quality Management, BusinessProcess-Reengineering, Balanced Scorecard etc.) nicht einfach über Bord wirft und dass gleichzeitig Altbewährtes auch nicht lediglich in neue Worte und Beschreibungen gefasst wird.

Auch kann in der gängigen betriebswirtschaftlichen Literatur festgestellt werden, dass nicht lediglich Organisationsstrukturen, sondern auch weitere Kriterien im Rahmen eines Wissensmanagements erfasst werden sollten, um eine Steigerung der Unternehmensleistung durch eine Wissensorientierung zu erzielen.

"[...] knowledge management encompasses broader issues and, in particular, creation of processes and behaviours that allow people to transform information into the organization and create and share knowledge. Thus, knowledge management needs to encompass people, process, technology and culture" (Kakabadse et al., 2001). Es muss zu einer Verknüpfung von informationstechnologischen mit organisatorischen Strukturen und der Unternehmenskultur erfolgen (vgl. Probst/Raub/ Romhardt, 1997). Im Rahmen der Betrachtung eines ganzheitlichen Wissensmanagements werden drei Bereiche (die Informations- und Kommunikationstechnologien, die Organisation sowie das Human Ressource Management) als Voraussetzung für ein erfolgreiches Wissensmanagement genannt (vgl. North, 1990; Gaßen, 1999; Schick, 2002; Pawlowsky, 1994; Albrecht, 1997). 
Die Unzulänglichkeiten der einzelnen Ansätze wurden bereits festgehalten. Dennoch kann nicht außer Acht gelassen werden, dass nicht lediglich Organisationsstrukturen, die die formalen Abläufe eines Unternehmens festhalten, ein Wissensmanagement ermöglichen können, sondern die Akteure eines Unternehmens eine nicht zu unterschätzende Rolle in der erfolgreichen Durchführung von Wissensmanagement spielen. Legt man einem Wissensmanagement-System Organisationsstrukturen als Fundament zugrunde, scheint es, als würde dem Individuum und den sozialen Faktoren eines Unternehmens keine adäquate Beachtung geschenkt. Durch die mikroperspektivistische Ausrichtung der Theorie der Neuen Institutionenökonomie wird dem entgegengewirkt. Denn eine Koordination und Motivation von Individuen kann nur dann erfolgreich durchgeführt werden, wenn diesen Gebieten ein besonderer Stellenwert zugesprochen wird.

Die bestehende betriebswirtschaftliche Literatur im Bereich Wissensmanagement, die sich mit der Ausgestaltung von Organisationsstrukturen beschäftigt, stellt zu einem überwiegenden Teil institutionalisiertes Wissen in den Mittelpunkt der Betrachtungen. "[...] work groups continually find inventive and resourceful ways to get things done when the world defies routine prediction. This sort of routinization becomes particularly important when rapidly changing environments continuously challenge routine" (Brown/Duguid, 2001, S. 52).

Institutionen bieten Unternehmen eine Struktur für das Verhalten an und bilden ein Rahmengerüst, in dem eine Weiterentwicklung, aber auch ein stabiles und sicheres Arbeiten möglich ist. Durch einen fortwährenden gesellschaftlichen und wirtschaftlichen Wandel unterliegen jedoch auch Institutionen einer Veränderung. So können Institutionen nicht nur als externe Beschränkungen gesehen, sondern sollten bei umbruchartigen Entwicklungsphasen als Chance wahrgenommen werden. Unsicherheiten, die dadurch auftreten mögen, lassen sich stabilisieren, sobald gewandelte Institutionen wieder etabliert wurden. Die Bedeutung politischer und sozialer Institutionen wurde sowohl von Ökonomen (beispielsweise Williamson, 1975), Politikwissenschaftern (beispielsweise March/Olsen, 1989), 
Soziologen (beispielsweise Powell/ DiMaggio, 1991) als auch Wirtschaftshistorikern (beispielsweise North, 1990) zunehmend in den Vordergrund gerückt.

Betrachtet man die Organisation aus einer institutionenökonomischen Perspektive, so stellen die Mitarbeiter des Unternehmens die Agenten dar, die die von den Prinzipalen definierten und vorgegebenen Aufgaben und Zielvorgaben zu erfüllen haben. Gleich beim Einstieg in ein Unternehmen werden dabei die Verfügungsrechte an den unternehmenseigenen Ressourcen klar abgeklärt und vertraglich vereinbart, so dass die institutionalisierten Verhaltensnormen in Bezug auf die Nutzung von Ressourcen zur Anwendung gelangen. „Ein Vertrag im ökonomischen Sinne ist jede bindende explizite oder implizite Vereinbarung über den Austausch von Gütern oder Leistungen zwischen Menschen, die dieser Vereinbarung zustimmen, weil sie sich davon eine Besserstellung versprechen" (Milgrom/Roberts, 1992, S. 127f).

Durch eine hierarchische Gliederung kann ein und derselbe Mitarbeiter in einer Arbeitskonstellation die Rolle des Agenten erfüllen und in einer anderen wiederum selbst der Prinzipal sein. Die Anweisungsmöglichkeiten und Machtstrukturen, die sich durch eine hierarchische Gliederung eines Unternehmens ergeben, werden bei Williamson als essentiell für die Überlebensfähigkeit von Firmen angesehen. So definieren sich die Rechte und Pflichten eines Mitarbeiters abhängig von seiner Arbeitsstelle und den damit einhergehenden Verfügungsrechten an Ressourcen. Organisationsstrukturen sollten die Beziehungs- und Machtstrukturen widerspiegeln, sodass Transaktionen günstig und effizient durchgeführt werden können. Diese Regelungen werden getroffen, um eine "optimale" Koordination in einem Unternehmen zu ermöglichen. $\mathrm{Da}$ in einem Unternehmen unter anderem aufgrund von ungleicher Informationsverteilung Interessensunterschiede auftreten werden, bieten Institutionen einen koordinierten Handlungsspielraum für die Akteure eines Unternehmens. Die Zusammenarbeit von Akteuren ist jedoch auch verbunden mit Gefahren, da aus dem Zusammenspiel von Unsicherheit, begrenzter Rationalität und dem Opportunismus ökonomischer Akteure falsche Anreize resultieren können. Das daraus folgende Organisationsproblem stellt in 
erster Linie ein Motivationsproblem dar und besteht darin, Institutionen auf eine Weise zu gestalten, dass unproduktives Verhalten von Mitarbeitern, das einerseits für sie selbst zu besonderen Vorteilen gereichen bzw. andererseits im Rahmen der Erreichung von Unternehmenszielen zu Nachteilen führen könnte, eingedämmt bzw. Koordinations- und Motivationskosten minimiert werden.

Die kritische Herausforderung, derer sich Unternehmen im Rahmen eines Wissensmanagement-Ansatzes gegenüber sieht, stellt die Grenze, die zwischen implizitem und explizitem Wissen gezogen wird, dar. Eine Beschreibung und Festlegung von Handlungsabläufen über formale Organisationsstrukturen wirkt sich positiv auf die Koordination von Mitarbeitern aus und das Erreichen von Unternehmenszielen wird unter Berücksichtigung der Senkung von Transaktionskosten realisierbar. Wie detailliert jedoch der Handlungsspielraum von Akteuren formalisiert werden und in Organisationsstrukturen manifest sein sollte, knüpft an die Frage, welchen Gestaltungsraum man Akteuren eines Unternehmens für ihre eigene Kreativität übrig lässt. Implizites Wissen wird mit Intuition, Geschicklichkeit, Kreativität etc. assoziiert, die in formellen Handlungsabläufen nicht festgehalten werden können. Werten Individuen die Daten, die sie in Folge von eigenen Erfahrungen, Gedankenkonstrukten, Beobachtungen sowie im Studium angesammelt haben, aus und ziehen sie Schlussfolgerungen, wie sie bestimmten Situationen am besten gegenübertreten sollen, so werden sie die ausgewerteten Daten in Handlungen überführen und anwenden. Die Gewissheit darüber, wie in bestimmten Situationen und Umständen gehandelt werden soll, lässt sie wissen, wodurch Individuen über Wissen verfügen. Dieses Personen inhärente Wissen stellt implizites Wissen dar. Es ist auf eine Weise vorhanden, dass man es zwar anwenden, jedoch diese Fertigkeit nicht beschreiben und begründen kann bzw. möchte, auch in dem Sinne "we know more than we can say" (Polanyi, 1962). Individuen werden dennoch Versuche unternehmen, das ihnen durch reine Intuition und reine Erfahrung eigene Wissen zu artikulieren, um andere an diesem Wissen teilhaben zu lassen. 
Diese Informationen, (keine Daten, da es sich ja um für ein Unternehmen relevante und kontextbezogene Daten handelt und wurden sogleich nach dem Prozess der Externalisierung zu Information), die nun durch diesen Transfer auf einer expliziten Ebene zur Verfügung stehen, können dann auf eine organisationale Ebene gehoben werden, indem sie über Verfahrensregeln im Unternehmen manifestiert werden. Solange diese Informationen von mehreren Personen geteilt und wiederum als eigenes Wissen internalisiert werden, kann noch nicht von explizitem organisationalen Wissen gesprochen werden. Vielmehr müssten Verfahrensweisen institutionalisiert werden, um als Lösungen für bestimmte Aufgabenstellungen weitgehend bekannt zu sein und unabhängig von Personen als Wissen existieren zu können. Institutionen weisen dadurch einen stark normativen Charakter auf. Sie sind nicht nur Antizipationen und Vorhersagen von Handlungen und legen nicht nur fest, wie Akteure handeln, sondern auch, wie sie handeln sollen (vgl. Scott, 1994). Explizites organisationales Wissen subsumiert somit institutionalisiertes Wissen.

Man erreicht durch eine Institutionalisierung, dass die Wissensverarbeitung nicht nur einzelnen Spezialisten und Abteilungen (wie beispielsweise der Abteilung für Forschung und Entwicklung) überlassen wird, sondern Aufgabe des gesamten Unternehmens und aller seiner Teile ist. Das Unternehmen schafft dadurch eine Streuung an Verantwortung, die an einen Akteur gestellten Arbeitsanforderungen mithilfe der institutionalisierten Handlungsabläufe zu erfüllen, und übergibt sie jedem einzelnen Mitarbeiter - unabhängig von seiner Position und Funktion. So kann auch nicht eine einzige Abteilung für sich alleine das Thema Wissen behandeln, sondern es werden vielmehr unternehmensweit Rahmenbedingungen geschaffen, die sämtliche wissensintensiven Prozesse (wie beispielsweise Wissensgenerierung, -teilung, -verarbeitung, -sicherung) professionell in Gang setzen und unterstützen. Hiervon sind die organisationalen Strukturen, Funktionen und Informationssysteme eines Unternehmens betroffen (vgl. Klimecki, 1999).

Mit der bewussten Strukturierung des individuellen expliziten Wissens hat eine Organisation die Möglichkeit, diese Informationen zu sichern und aufzybewahren, 
damit sie schlussendlich institutionalisiert und auf organisationaler Ebene zu Wissen werden können. Alleine dieser Schritt berührt jedoch mehrere Bereiche, denen Beachtung geschenkt werden sollte und in anschließenden Kapiteln auch werden soll, wie beispielsweise die Rolle des Individuums oder die für eine effiziente Externalisierung notwendige technologische Fundierung.

Die Grenzen des Managements kommen an dieser Stelle ebenfalls zum Tragen, denn setzt man sich mit der Integration von Individuen in Organisationen näher auseinander, muss man klar abstecken, welche Aspekte eines Individuums für eine Organisation zugänglich und steuerbar sind bzw. sein soliten. Bereits an früherer Stelle wurde darauf hingewiesen, dass es kritisch und problematisch ist, den Menschen in seiner Gesamtheit in die Betrachtung mit einzubeziehen. Das Management orientiert sich daran, ein System an koordinierten Handlungen zu bieten, wobei die gesamtunternehmerischen Prozesse und Funktionen in einer Leistungserstellung resultieren. Personen als ausführende oder dispositive Mitarbeiter eines Unternehmens sind für die Aufgabenerledigung und die Funktionserfüllung verantwortlich, sie sammeln und verarbeiten Informationen und treffen Entscheidungen. Personen werden sich jedoch nur dann an einer Organisation beteiligen, wenn sie ihren eigenen Nutzen damit steigern können und wenn sich dadurch ihre individuellen Ziele besser erreichen lassen. Im Allgemeinen kann jedoch nicht davon ausgegangen werden, dass der gemeinsame Zweck eines Unternehmens unbedingt mit den Vorstellungen der Mitarbeiter korrespondiert (Arrow, 1985). Regelungen sollten auf eine Weise getroffen werden, dass Akteure wissen, welche Handlungsoptionen sie ausführen sollen und wissen, wie sie dies tun können. Des Weiteren sollten sie das, was sie tun sollen, auch wollen.

So ist es innerhalb eines Unternehmens von enormer Bedeutung, den gesamtunternehmerischen Zweck sowie die zu erreichenden Zielvorstellungen klar zu kommunizieren, um sicherzustellen, dass diese im Unternehmen bekannt sind und dadurch erreicht werden können. Hier fließen Ansätze des ManagementKonzepts der Balanced Scorecard ein, im Zuge deren ebenfalls zu Beginn die Vision eines Unternehmens definiert wird um darauf aufbauend die strategische 
Ausrichtung eines Unternehmens festzusetzen (Kaplan/Norton, 1996). Auch Nonaka/Takeuchi (1996) setzen die Unternehmensintention vor allen anderen unternehmerischen Aktivitäten an die erste Stelle, was sich als das Streben des Unternehmens nach seinen Zielen definieren lässt. Wird es verabsäumt Ziele darzulegen, wird in einer Organisation kaum jemand wissen, welche Leistungen vom Einzelnen verlangt werden. „Jedes Glied des Unternehmens leistet einen besonderen Beitrag; aber einen Beitrag zum gemeinsamen Ziel müssen alle leisten. Alle Anstrengungen müssen in der gleichen Richtung zielen und sich so erhängen, dass ein Ganzes entsteht - ohne Lücken, ohne Reibungen, ohne überflüssige Doppelarbeit" (Drucker, 1956, S. 153).

Durch das Aufzeigen von Zielfunktionen alleine erhält man jedoch noch keine Gewähr, dass unbedingt gewusst wird, auf welche Art und Weise diese am besten erreicht werden. So ist es ebenfalls von einer besonderen Wichtigkeit, die dazu notwendigen Handlungsabläufe klar darzustellen (hier fließt der Ansatz der "best practices" ein). Diese werden in einzelne Verantwortungsbereiche zusammengefasst, sodass jeder Mitarbeiter seine Leistungen und Beiträge auf ein Unternehmensganzes ausrichten kann.

Durch eine solche funktionale Gliederung eines Unternehmens kann erreicht werden, dass sich Mitarbeiter nicht nur von ihrer Arbeitsstelle in eine Abteilung, sondern auch von ihrer Abteilung her in ein Unternehmensganzes einordnen können. Denn durch die Anordnung und Ausrichtung der Funktionen einer Organisation wird der direkte Weg aufgezeichnet, wie ein Unternehmenszweck und Unternehmensziele erreicht werden können. Eine aufbauorientierte Organisationsform greift jedoch im Rahmen eines Wissensmanagements zu kurz und muss um eine ablauforientierte Perspektive erweitert werden. Somit soll ein Übergang zu dem Management-Konzept des Business Reengineering (vgl. beispielsweise Hammer, 1990; Champy, 1995) bzw. des Business Process Redesign (vgl. Davenport/Short, 1990) geschaffen werden, die eine dynamische und prozessorientierte Perspektive in einem Unternehmen gewährleisten. 
Auch wenn Mitarbeiter grundsätzlich ihre Verantwortlichkeiten kennen, so müssen sie darüber informiert werden, welche spezifischen Arbeiten von ihnen zu erledigen sind, in welche Beziehungen sie damit zu anderen Mitarbeitern und Abteilungen treten, welche Zielsetzungen sie zu erfüllen haben und welchen Arbeitsstellenzweck sie dabei verfolgen, womit sie direkt die Ziele sowie den Zweck des gesamten Unternehmens zu erreichen helfen.

Das Unternehmen sollte Mitarbeiter Anreizstrukturen in der Form bieten, dass sie motiviert genug sind, ihre Arbeiten gut zu erledigen, damit sie das Unternehmen unterstützen, seine Ziele zu erreichen. Dabei müssen Mitarbeiter sehr oft abteilungsübergreifend tätig werden und hierbei ist es von enormer Wichtigkeit, dass Mitarbeiter bei der Durchführung ihrer Arbeiten genau wissen, von wem sie die für eine Aufgabenstellung notwendigen Informationen erhalten, mit wem sie gewisse Schritte gemeinsam durchführen und an wen sie selbst bestimmte Informationen weiterleiten müssen. Dadurch sollten die Beziehungs- und Kommunikationsstrukturen eines Unternehmens bekannt sein unabhängig von der jeweils zu beantwortenden Fragestellung bzw. dem zu lösenden Problembereich.

Ein weiterer Faktor, der das Schaffen eines Wettbewerbsvorteils gegenüber der Konkurrenz stark beeinflusst, stellt die Geschwindigkeit dar, mit der die einzelnen Handlungsabläufe durchgeführt werden. Dies ist vor allem von der Motivation der Mitarbeiter, den Kommunikationsstrukturen und auch teilweise von den zugrunde gelegten Informationstechnologien (die jedoch wiederum die Kommunikationsstrukturen unterstützen) abhängig.

Zusätzlich ist institutionalisiertes Wissen einem Wandel unterlegen (North, 1990), dem insofern entsprochen werden muss, als unter Umständen die Organisationsstruktur selbst einer Veränderung bedarf oder als die Verfahrensweisen überarbeitet werden müssen. Daher sollten auch die Verträge mit den einzelnen Mitarbeitern auf eine Weise gestaltet sein, dass das jeweilige Unternehmen flexibel auf interne Umstrukturierungen und Verbesserungsvorschläge oder aber auf Umweltveränderungen reagieren kann. Durch die Langfristigkeit der formalen 
Verträge besteht für ein Unternehmen die Möglichkeit, die sich ändernden Faktoren über informelle Verträge zu erweitern bzw. zu adaptieren. Da dies wiederum Gefahren mit sich bringt aufgrund der zugrunde gelegten Verhaltensannahmen wie begrenzter Rationalität und Opportunismus sowie aufgrund einer ungleichen Informationsverteilung werden auch die Anreiz- und Sanktionsmechanismen in einem Unternehmen einem Wandel unterliegen.

Verbesserungen, interne Umstrukturierungen und sämtliche andere unternehmerische Veränderungen können jedoch lediglich von Individuen angeregt werden, sodass die Bedeutung der Förderung jedes einzelnen Mitarbeiters nochmals hervorgehoben werden soll (vgl. auch bspw. Nonaka/Takeuchi, 1995). Da dieser Punkt eine derartige Wichtigkeit besitzt und langfristig über die strategische Positionierung eines Unternehmens entscheiden kann, könnte im Unternehmen eine eigene Qualitätsabteilung integriert werden, um den Aspekt Qualität, der "Wissen verfeinern" inkludiert, zu institutionalisieren. Dies stellt einen Schlüsselpunkt des hier erarbeiteten Wissensmanagement-Ansatzes dar. Es fließen Annahmen der Konzepte Total Quality Management, des Organisationalem Lernens sowie der Balanced Scorecard ein.

Bevor Mitarbeiter jedoch überhaupt Verbesserungsvorschläge einbringen werden, muss eine Basis geschaffen sein, dass Akteure eines Unternehmens die innen zugewiesenen Arbeitsbereiche und Aufgabenstellungen erledigen wollen. Sind sie darüber hinaus motiviert, die Leistungserstellung in einem Unternehmen zu verbessern, werden sie ihr Wissen externalisieren und im Unternehmen zur Diskussion stellen, um in weiterer Folge Prozesse zu initiieren, die Handlungsabläufe in einem Unternehmen verändern und verbessern helfen. Die einzelnen Mitarbeiter zu motivieren und zu fördern, soll sich in diesem Wissensmanagement-Ansatz klar und deutlich im Einflussbereich der Personalabteilung befinden.

Voraussetzung für die Realisierung dieses Wissensmanagements würden ein allgemein bekannter Unternehmenszweck und darauf aufbauende Unternehmensziele sein, die von den einzelnen Abteilungen erreicht werden sollen. Die 
Mitarbeiter der einzelnen Abteilungen wüssten auf Grund von Arbeitsplatzbeschreibungen, welche Aufgaben in ihren Verantwortungsbereich fallen und durch die institutionalisierten Verfahrensregeln, wie und mit wem sie diese Aufgaben zu erledigen haben. Durch Weiterbildungsmaßnahmen der Qualitätsabteilung und durch ständigen Kontakt mit der Personalabteilung würden sie in das Unternehmen auf eine Weise integriert, dass sie die Motivation für Verbesserungsvorschläge, innovative Ideen und ähnliches aufbringen. Dadurch hat externalisiertes, individuelles Wissen die Möglichkeit institutionalisiert zu werden, wodurch ein Unternehmen an Wissen und Wettbewerbsvorteilen gewinnt.

Im Zuge der Festlegung der für das jeweilige Unternehmen passenden Organisationsstruktur werden neben den Produktionskosten, die sämtliche Kosten subsumieren, die für die unmittelbare Leistungserstellung anfallen, auch Koordinationsund Motivationskosten berücksichtigt (Picot/Diet//Franck, 1997). Letztere sollen in diesem Wissensmanagement-Ansatz durch die besondere Bedeutung, die der Personalabteilung zugesprochen wird, sowie durch die organisatorische Ergänzung einer Qualitätsabteilung reduziert werden. Es wird davon ausgegangen, dass die Senkung der Produktionskosten durch eine Erhöhung der Koordination und Motivation von Mitarbeitern gewährleistet werden kann, was sich unmittelbar auf den Wettbewerbsvorteil eines Unternehmens auswirkt.

Legt man Organisationsstrukturen und institutionalisierte Handlungsabläufe einem Wissensmanagement-Ansatz zugrunde, so wird explizitem Wissen in einem Unternehmen eine Schlüsselrolle zugesprochen. Dies würde, rekurriert man auf die Ausführungen von Lam (2000), bedeuten (vgl. auch das Kapitel "Wissen managen - eine symbiotische Beziehung oder ein Widerspruch in sich?"), dass sich eine "professional bureaucracy" bzw. eine "machine bureaucracy" als eine passende Organisationsform darstellen würde. $\mathrm{Da}$ in diesem WissensmanagementAnsatz jedoch dem Individuum eine besondere Bedeutung zugesprochen wird und das sich inm zu eigene implizite Wissen als nicht weniger relevant als explizites Wissen darstellt, kann auch keine der beiden oben genannten Organisationsformen den Anforderungen dieses Wissensmanagement-Anşatzes genügen. 
Wird der Schwerpunkt eines Unternehmens auf das implizite Wissen gelegt, so werden die "operating adhocracy" bzw. die "j-form-organization" empfohlen (Lam, 2000). Doch auch diese Formen stellen sich als unzulänglich für die Anwendung im Zuge des vorliegenden Wissensmanagement-Ansatzes dar.

Mithilfe eines vertragsbasierten Unternehmens, das mittels seiner Organisationsstrukturen institutionalisierte Handlungsabläufe anbietet und somit explizites individuelles Wissen auf eine organisationale Ebene bringen kann und gleichzeitig der Motivation und Koordination von Mitarbeitern Aufmerksamkeit schenkt, wodurch die Akteure eines Unternehmens bereit sein sollten, das ihnen zu eigene implizite Wissen im Sinne des Unternehmens trotz unterstellter begrenzter Rationalität, zugrunde liegenden Opportunismus und Informationsasymmetrien zu externalisieren und für eine Verbesserung der Handlungsabläufe einzusetzen, wird beiden Arten von Wissen sowie deren Wechselspiel eine gleichbedeutende Rolle zugesprochen.

Es wird gewährleistet, dass Wissensmanagement nicht an einer Stelle eines Unternehmens versickert, sondern eine Querschnittsfunktion erfüllt. So bedarf es bei der Durchführung von Wissensmanagement nicht einer radikalen Umstrukturierung eines Unternehmens, sondern unter anderem des Bewusstwerdens darüber, dass Unternehmen wahrscheinlich bereits weitaus mehr Wissen managen, als sie sich erwartet hätten. Um das Managen von Wissen strukturiert und im Sinne eines erfolgreichen Wissensmanagements durchzuführen, wird in der vorliegenden Arbeit empfohlen, das bereits vorhandene "Wissensmanagement" mit den hier getätigten institutionellen Schwerpunktsetzungen in Form einer Personal- und Qualitätsabteilung, die den Fokus auf das Wissen der Mitarbeiter legen, zu erweitern. 


\subsection{Die Rolle des Individuums}

Mitarbeiter können alles: wenn man sie weiterbildet, wenn man ihnen Werkzeuge gibt, vor allem aber, wenn man es ihnen zutraut.

Hans-Olof Henkel

Mitarbeiter werden über formale und informelle Verträge an das jeweilige Unternehmen gebunden. Nach erfolgreicher Einschulung in den Aufgabenbereich und nach einer Gesamtübersicht über die Organisation ist ein Mitarbeiter positioniert und orientiert, sodass er sich als ein Teil eines Ganzen versteht, wo seine Arbeit direkt in der Erfüllung der Unternehmensziele und des Unternehmenszweckes resultiert. Er hat Kenntnis davon, mit wem er in gewissen Arbeitssituationen zusammenarbeiten, von wem er für welche Aufgabenstellungen Informationen erhalten und wem er selbst Informationen geben kann/soll.

Diese Zusammenarbeit gelingt jedoch nur in einem solchen Ausmaß, wie Mitarbeiter selbst daran interessiert sind, dass eine Koordination und Kooperation in der Form stattfindet, wie es von dem jeweiligen Unternehmen vorgesehen ist. So wird das Verhalten jedes Mitarbeiters, das von einem Unternehmen gewünscht und für die Erreichung der Unternehmensziele auch gebraucht wird, so weit wie möglich auf formalisierte Weise in Verträgen festgehalten, in die auch Steuerungs-, Anreiz-, Kontroll- und Informationsmechanismen integriert werden. Zusätzlich dazu stellt jedoch ein anderer Faktor eine zentrale Bestimmungsgröße menschlichen Handelns dar und übt entscheidenden Einfluss auf die erbrachte Leistung von Mitarbeitern aus. Dies ist Motivation, ein Begriff der als Bereitschaft eines Mitarbeiters definiert werden kann, eine Leistung zu erbringen bzw. ein bestimmtes Verhalten zu zeigen. Für ein Unternehmen ist es nicht möglich, alle betrieblichen Abläufe und Entscheidungen bis ins kleinste Detail vorherzusagen und festzulegen, wodurch eine Formalisierung der Unternehmensaktivitäten lediglich bis zu dem Grad erfolgen kann, ab dem die Erreichung der Unternehmensziele als sichergestellt gelten. Sämtliche Handlungen, die darüber hinaus erforderlich sind, um die Unternehmensziele und den Unternehmenszweck tat- 
sächlich zu erfüllen, finden sich in einem Gestaltungsbereich der Mitarbeiter selbst und fordern das implizite Wissen, Eigenengagement, Kreativitätspotential und die Selbstinitiative jedes einzelnen Mitarbeiters (zwar in unterschiedlichem Ausmaß, da dies von der jeweiligen Arbeitsstelle und dem Aufgabenbereich mit dazugehörigen Verantwortlichkeiten abhängig zeichnen wird).

Im Rahmen von vertraglichen Abkommen gehen Mitarbeiter ein Abhängigkeitsverhältnis mit einem Unternehmen ein. Sie erhalten zwar von ihrem Auftraggeber bestimmte Aufgaben und Entscheidungskompetenzen übertragen und eine Vergütung für ihre Leistungen, jedoch unterliegt ihr Handlungsspielraum auf Grund von auftretenden Interessensunterschieden und Informationsasymmetrien gewissen Steuerungs- und Kontrollmechanismen. Es wird davon ausgegangen, dass Mitarbeiter über spezifische Kenntnisse und Fertigkeiten verfügen, die innen einen Informationsvorsprung gegenüber ihrem Auftraggeber bringen („moralisches Risiko"). Ein Auftraggeber sieht sich nun in einer Situation wieder, in der er gewährleisten muss, dass der jeweilige Mitarbeiter seinen Informationsvorsprung nicht auf Kosten des Unternehmens und lediglich für seine eigenen Interessen einsetzt, sondern im Sinne des gesamten Unternehmens agiert. Durch die Institutionalisierung von Handlungsabläufen kann man dieser "hidden information" entgegensteuern. Es ist im Sinne des Unternehmens, dass Mitarbeiter ihre Individualität behalten und Arbeiten auch auf ihre eigene Art und Weise erledigen. Lediglich in Situationen, in denen dieser Informationsvorsprung dadurch, dass er nicht in Organisationen einfließt, zu einem Nachteil gereicht, kommen GovernanceMechanismen zum Einsatz.

Die organisationsinterne Leistungserstellung ist aufwendig zu gestalten, wenn sichergestellt werden soll, dass sämtliche Mitarbeiter eines Unternehmens ihre Tätigkeiten in eine Richtung ausrichten. So regeln starke Anreizmechanismen nicht unmittelbar die effiziente Abwicklung von Transaktionen, wie dies bei marktlichen Austauschbeziehungen der Fall ist, da die Leistungen und Gegenleistungen in organisationsinternen Austauschbeziehungen infolge von Mess- und Zurechnungsproblemen oft nicht unmittelbar und eng gekoppelt sind Es wird ver- 
sucht, Anreizmechanismen des Marktes intern zu simulieren und die bestehenden Anreizdefizite zu kompensieren.

Eine weitere Implikation der Probleme der Leistungsmessung besteht darin, dass Mitarbeiter versuchen können, weniger Leistung zu erbringen bzw. andere Ressourcen als vereinbart in die Leistungserstellung einfließen zu lassen. Es knüpft an die ungleiche Informationsverteilung zwischen Mitarbeitern und Vorgesetztem an, wobei letzterer über die genauen Handlungsmöglichkeiten und das tatsächliche Leistungsverhalten seiner Mitarbeiter keine genaue Kenntnis haben kann. Ein derartig offener Handlungsspielraum ermöglicht grundsätzlich Mitarbeitern, verschiedene Vorgehensweisen (Mittel) zur Zielerreichung zu wählen („hidden action"). Das Ergebnis ist für einen Prinzipal dann zwar ex-post feststellbar, jedoch lassen sich keine sicheren Rückschlüsse auf die ex-ante-Entscheidungssituation und die Leistungsanstrengungen des jeweiligen Mitarbeiters ziehen.

Auch die beschränkte Beobachtbarkeit seines Verhaltens kann einen Mitarbeiter dazu verleiten, seine Leistung zu reduzieren („shirking“) oder Unternehmensressourcen für eigennützige Zwecke zu verwenden. Ebenfalls zu einem ineffizienten Ergebnis führt nicht-kooperatives Verhalten, wenn sich Mitarbeiter die gemeinsame Ausbringung vollständig teilen müssen (Holmstrom, 1982). Das potentiell auftretende opportunistische Verhalten einzelner Mitarbeiter intendiert ein bewusstes Verschweigen, Verschleiern und Verzerren von Informationen (Williamson, 1989). Um einem solchen Verhalten entgegenzuwirken, stehen einem Prinzipal mehrere Möglichkeiten offen:

Es kann versucht werden, Anreizstrukturen auf eine Weise zu integrieren, die einerseits zur Erfüllung der Ziele eines Mitarbeiters und andererseits zur Erfüllung der Unternehmensziele führen (Laux, 1990; Laux/Schenk-Mathes, 1992). Eine Ergebnisbeteiligung durch Mitarbeiter kann beispielsweise in diese Richtung lenken. Durch die Zusammenführung von Zielvorstellungen reduziert man das Zielkonfliktpotential, da die Erträge für beide Parteien von den gleichen Leistungen abhängig sind. Es muss jedoch gewährleistet werden, dass das Ergebnis 
tatsächlich auf den messbaren Leistungsbeitrag eines Mitarbeiters und nicht etwa auf Umwelffaktoren oder den Leistungsbeitrag eines anderen Mitarbeiters zurückzuführen ist. Auch das Risiko, das mit einer Leistungserstellung einhergeht, kann nicht lediglich ein Prinzipal tragen, sondern muss sich auf die an einer Leistungserbringung beteiligten Personen aufteilen. So schließt die optimale Gestaltung von ergebnisorientierten Verträgen einen Kompromiss in der Verteilung der Ergebnisse und des Risikos mit ein (Hartmann-Wendels, 1989; Shavell 1979).

Governance-Mechanismen bieten nicht nur Anreizstrukturen, sondern können auch eine direktive Verhaltenssteuerung unterstützen. So wird vertraglich der Handlungsspielraum jedes einzelnen Mitarbeiters klar abgesteckt, dessen Einhaltung kontrolliert und dessen negative Verletzung sanktioniert. Aus der Perspektive eines Motivationsproblems seitens eines Mitarbeiters ist die Überwindung des Nichtwollens im Rahmen arbeitsteiliger Leistungserstellungsprozesse mit Kosten verbunden, da alleine die Etablierung der Governance-Mechanismen Kosten verursacht (Jensen/Meckling, 1976). Instruktive Normen setzen voraus, dass ein Prinzipal über einen ähnlichen, wenn nicht besseren, Informationsstand als seine Mitarbeiter verfügt, was jedoch bei komplexen und unstrukturierten Aufgaben kaum möglich ist. Auch wenn das interne Informationssystem verbessert wird, wodurch ein Prinzipal die Möglichkeit gewinnt, sich mehr Wissen über das Leistungsverhalten und die Handlungssituation seiner Mitarbeiter anzueignen, haben grundsätzlich positive Leistungsanreize eine höhere Durchsetzungswahrscheinlichkeit und bieten eine kostengünstigere Verhaltenssteuerung (Laux, 1990).

Damit derartige beherrschende Strukturen etabliert werden können, muss es jedoch ein ein- oder wechselseitiges Abhängigkeitsverhältnis zwischen den Akteuren geben. In Form eines Arbeitsvertrags wird eine Leitungsmacht legitimiert und die Macht der einzelnen Akteure ergibt sich durch eine systeminterne Bewertung ihrer Ressourcenausstattung bei vorausgesetzten Verfügungsrechten. Betrachtet man auf der anderen Seite die Entstehung von Rechten, wird auch eine umgekehrte Kausalität festgestellt. So werden Verfügungsrechte akzeptiert, indem ein Konsens gebildet und eine "allgemeine Tendenz, die Sichtweişen ver- 
schiedener Personen über die Verteilung von Rechten mit der Zeit in Übereinstimmung zu bringen" (Colemann, 1991, S.63), postuliert wird, die sich letztlich aus der Verteilung der Macht zwischen den beteiligten Akteuren ergeben. (Die verschiedenen Aspekte von Macht werden beispielsweise bei Kappelhoff, 1997 oder Colemann, 1991 weiter ausgeführt).

Dennoch werden Machtstrukturen und formale Sanktionsmechanismen nicht ausreichen, um ein gewünschtes Verhalten bei den Mitarbeitern hervorzurufen. Ein integratives Modell von Motivation und Leistung findet sich beispielsweise bei Mayerhofer (2002). Während herkömmliche Formen der leistungsbezogenen Entlohnung vielfach auf extrinsischer Motivation aufbauen, gewinnt für die Wissensteilung und Wissensentwicklung die intrinsische Motivation an Bedeutung (Frey/Osterloh, 2000). Auch der Zuschnitt des Arbeitsplatzes (,job design“) stellt ein wichtiges Instrument zur Steuerung der Anreize dar. Die Vorstellung, dass jeder einzelne Mitarbeiter lediglich eine Aufgabe übernimmt, greift zu kurz (Baker/Jensen/Murphy, 1988). Die interdisziplinäre Methodologie der Neuen Institutionenökonomie ermöglicht die Integration von "weichen" organisatorischen Steuerungsmedien und berücksichtigt, dass „Unternehmen wie alle Organisationen Formen personaler Kooperation darstellen, für die die Bedeutung von kulturellen und normativen Faktoren konstitutiv sind“" (Wieland, 1997, S. 37).

Die Anschlussfähigkeit ist hierbei vor allem von einem gemeinsamen Werteverständnis sowie von einer gemeinsamen Denkausrichtung abhängig, die sich in Unternehmenskulturen findet, die als Grundgesamtheit gemeinsamer Wert- und Normenvorstellungen sowie geteilter Denk- und Verhaltensmuster verstanden wird und die Entscheidungen, Handlungen und Aktivitäten der Mitarbeiter eines Unternehmens prägt. Ihr wird als Rahmen und auch Erfolgsfaktor eine besondere Bedeutung zugesprochen (vgl. Güldenberg, 1998) und die erfolgreiche Integration von Wissensmanagement stellt sich als eine Konsequenz der kulturellen Verankerung wissensfördernder Denk- und Handlungsweisen dar (vgl. beispielsweise North, 2000; Probst/Raub/Romhardt, 1997; Kalseth, 2001). Ein gemeinsa- 
mes Werte- und Normensystem der Unternehmensmitglieder unterstützt den internen Organisationsprozess (Picot/Diet//Franck, 1997).

Dieses Streben nach mehr Wissen bzw. nach einer Wissensteilung und gemeinsamer Wissensgenerierung fruchtet ausschließlich in einer Umgebung, in der eine kreative Zusammenarbeit möglich ist und in der Mitarbeitern ein Handlungsspielraum eingeräumt wird, in dem auch Fehler erlaubt werden. Vertrauen muss sichtbar und immer und überall gegeben sein sowie die Vertrauenswürdigkeit einer Person als Tugend an oberster Stelle stehen (Davenport/Prusak, 1998). Jedoch "Vertrauen" ist ein Begriff, der vielfältig verwendet wird und nur selten findet sich ein Konsens, was alles unter diesem Konzept subsumiert werden kann. Unterschiedliche Ansätze werden beispielsweise von Huemer/von Krogh/Roos (1998) oder Kramer/Tyler (1996) aufgearbeitet.

Die Personalabteilung, als institutionalisierte Instanz, soll in diesem Wissensmanagement-Ansatz dafür verantwortlich zeichnen, dass Mitarbeiter das vom Unternehmen gewünschte Verhalten auch erbringen und erbringen wollen. Dies inkludiert die Gewährleistung, dass die Mitarbeiter die institutionalisierten Handlungsabläufe einhalten, im Rahmen der formalen, vertraglich festgelegten Verhaltensregelungen agieren und dennoch genug Freiräume besitzen, um ihrer Kreativität und ihrem Ideenreichtum nachgehen zu können und diesen Gestaltungsspielraum zu ihrem eigenen Vorteil und dem des Unternehmens zu nutzen. 


\subsection{Kommunikation - der Schlüsselfaktor}

\section{Organisationales Wissen ist nicht gänzlich und insgesamt von Personen un-}

abhängig,

denn es ist in der kommunikativen Aktivierung immer auf irgendwe/che Per-

sonen angewiesen.

Willke

Kommunikation wird im Zuge eines Wissensmanagements eine besondere Bedeutung zugesprochen. Diese Schwerpunktsetzung kommt zwar in einem interpretativen Paradigma weit mehr und deutlicher zum Ausdruck als in Konzepten einer funktionalistischen Logik, dennoch kann sie als Fundament von Austauschprozessen zwischen Akteuren nicht wegrationalisiert werden und unterliegt daher einer näheren Analyse. Kommunikation wird dabei in mündlicher, jedoch unterstützt durch die neuen Medien im Bereich der Informatikwissenschaften vermehrt auch in schriftlicher Natur erfolgen (vgl. Venters, 2002).

Kommunikation spielt eine wichtige Rolle bei der Führung und Motivation von Mitarbeitern und somit bei der Realisierung von Unternehmenserfolgen. Sie kann auf verschiedenen Ebenen - beispielsweise auf individueller Basis, auf Basis der Gruppe oder aber der Organisation - stattfinden und jede Ebene ist Gegenstand unterschiedlicher Analysen diverser Forschungstraditionen ( $\mathrm{vgl}$. Jablin/Sias, 2001). Auf Mikroebene kommt es zu Ausführungen, die das Individuum mitsamt seinem Verhalten und seinen Fertigkeiten betrachten, wobei hierbei beispielsweise auf die folgende Definition von Kommunikation zurückgegriffen wird: „Kommunikation [...] kann, ebenso wie Interaktion, als ein Handlungsprozess betrachtet werden, der zwischen zwei oder mehreren Subjekten bzw. Akteuren abläuft, besonderen "Spielregeln" folgt und der Lösung bzw. Befriedigung von bestimmten Zielen gerecht zu werden versucht" (Stockinger, 1989, S. 78). Diese Definition wird der Arbeit zugrunde gelegt, im Bewusstsein, dass sich ein einheitliches Verständnis zum Kommunikationsbegriff in der Literatur nicht findet (vgl. beispielsweise Maletzke, 1998). 
Führen Mitarbeiter ihre Arbeiten aus, benötigen sie oftmals Informationen von anderen Bereichen des Unternehmens bzw. haben gewisse Aufgabenstellungen mit Kollegen durchzuführen. Kommunikationsnetzwerke innerhalb eines Unternehmens sollten hierbei von Organisationsstrukturen nachgezeichnet werden. In der Literatur wird beispielsweise zwischen dezentralen und zentralen Kommunikationsnetzwerken unterschieden (vgl. Rosenstiel, 1987). Erstere erlauben jedem Mitarbeiter mit jedem anderen in Kontakt zu treten, ohne eine zentrale Schaltstelle berücksichtigen zu müssen. Sämtliche Mitarbeiter befinden sich in derselben Ausgangsposition, mit gleichwertigen Kommunikationsmöglichkeiten. Das Gegenteil ist in letzteren Netzwerken der Fall, wo es sehr wohl eine zentrale Stelle gibt, über die Kommunikation laufen muss.

Der Aufbau einer Organisationsstruktur mit dazugehörigen Handlungsabläufen, die regeln, wie in einem bestimmten Unternehmen Aufgaben am besten erledigt werden und optimal für eine Zielerreichung und Erfüllung eines Unternehmenszweckes fungieren, ist relativ einfach zu gewährleisten, auch wenn Mühen und Zeit damit einhergehen, um sämtliches dafür notwendige individuelle Wissen auf eine organisationale Ebene zu bringen. Viel mehr Aufmerksamkeit sollte jedoch darauf gelegt werden zu gewährleisten, dass Mitarbeiter diese Strukturen auch im Zuge ihrer Arbeiten verwenden und verwenden wollen.

Betrachtet man Organisationen, so können auf struktureller Ebene Mechanismen eingebaut werden, die die Mitarbeiter dazu anhalten, die etablierten Kommunikationsstrukturen zu verwenden. Durch eine Unwissenheit und Unsicherheit seitens der Mitarbeiter werden jedoch Koordinations- und Motivationsprobleme auftreten, da sie nicht wissen, welche Handlungsoptionen sie zur Erledigung der an sie gestellten Arbeitsanforderungen wählen sollen. Es handelt sich hierbei um ein Entscheidungs- und Planungsproblem, das in der Überwindung der Unsicherheit in Bezug auf das ${ }_{n}$ richtige" Handeln liegt. Einem Unvermögen begegnen Akteure dann, wenn sie nicht in der Lage sind, das Ergebnis zu erzielen, das für ihre Arbeitsstelle vorgesehen ist (vgl. Scheuble, 1998). Mit routinisierten Kommunikationsstrukturen kann diesen Problemfeldern entgegen gewirkt werden 
Es zeichnet von enormer Wichtigkeit, dass Mitarbeiter gut darin eingeschult sind, welche Aufgabenstellungen sie in der jeweiligen Organisation zu erfüllen haben, dass sie die fachlichen Qualifikationen für ihre Stelle wirklich erbringen, dass sie darüber informiert sind, wie die Kommunikationsstrukturen eines Unternehmens im Allgemeinen laufen und wie sie sich spezifisch auf ihre Arbeitsstelle und ihren Aufgabenbereich darstellen, mit welchen Personen aus welchen Abteilungen zusammengearbeitet werden wird und welche Informationen auf welche Weise ausgetauscht werden.

Als Kern erfolgreichen Arbeitens sollte von allen Mitarbeitern eines Unternehmens ein gewisser Wissensbestand geteilt werden ("Von allen geteiltes Wissen“ Pautzke, 1989). Neben Daten über die Organisation selbst werden sich Mitarbeiter besser orientieren können, wenn sie die funktionale Gliederung eines Unternehmens, Handlungsabläufe etc. verstehen, indem sie wissen, dass diese in Richtung des Unternehmenszwecks führen und dass es dadurch Sinn macht, diese Strukturen zu verwenden und einzuhalten. Institutionen bieten hierfür den Rahmen, in dem die eingebetteten Daten und somit relevanten Informationen Anwendung finden. Dieses Wissen stellt vor allem Wissen dar, das ständig benötigt wird sowie als Strukturierungshilfe für speziellere Anwendungsfälle eingesetzt werden kann. Bei Willke (1996) unter "Allgemeinwissen" subsumiert und etwas erweitert enthält es fünf Dimensionen: die sachliche Dimension, die Kenntnisse über Organisationsveränderungen und deren Auswirkungen auf die Handlungen der Mitarbeiter beinhaltet. Dies weist auf den Charakter des Wandels von Institutionen hin. Das personale Wissen (soziale Dimension) kommt dem "von allen geteilten Wissen" bei Pautzke (1989) am nächsten und umfasst Informationen über Mitarbeiter, Kunden und Wettbewerber eines Unternehmens; schließt somit den Personenkreis um ein Unternehmen herum. Die zeitliche Dimension geht auf das Prozesswissen ein und somit werden Zeitanforderungen an die Mitarbeiter gleich zu Beginn abgesteckt. Einen Schlüsselpunkt weist die operative Dimension auf, wo Mitarbeiter darin eingeschult werden, welche Verfahrensweisen und Standardinstrumente in einem Unternehmen bestehen, sowie die kognitive Di- 
mension, die das Wissen über die Identität und Zielsetzungen des Unternehmens umfasst.

Die funktionale Gliederung eines Unternehmens spiegelt die formalen Kommunikationsstrukturen wider (eine Kritik an einer funktionalistischen Positionierung findet sich beispielsweise bei North, 2002). Hierarchien zeigen auf, wie man in diesen Strukturen positioniert ist. Sind zusätzlich dazu die in einem Unternehmen notwendigen Handlungsabläufe für die Erfüllung der Unternehmensziele und des Unternehmenszweckes institutionalisiert, so könnte auf diese Weise gewährleistet werden, dass jeder Mitarbeiter darüber informiert ist, von welchen Personen er Informationen zur Bearbeitung von gewissen Aufgabenstellungen erhält, sowie an welche Personen er selbst zu deren Unterstützung Informationen weiterleiten bzw. mit wem er gemeinsam an einem Sachverhalt arbeiten wird.

"[...] the key to organizational communication focuses on the arrangement and structure of how information is channelled to the specific individuals and groups who need it for task, problem-solving, control, or decision-making purposes. In fact, the way a group or organization is structured ultimately determines the accessibility of an ease with which members can communicate with one another" (Bowditch/Buono, 1990, S. 116). Dies streicht die Bedeutung einer klaren Strukturierung der Kommunikations- und Austauschbeziehungen innerhalb eines Unternehmens hervor, jedoch wird sie nicht alleine ausreichen, um langfristig einen komparativen Vorteil gegenüber Mitbewerbern zu generieren. Denn erst die tatsächliche Nutzung und die für eine Aufgabenerledigung benötigte Geschwindigkeit werden darüber entscheiden, ob man sich am Markt besser als die Konkurrenz positioniert oder nicht.

Kommunikation nimmt daher innerhalb eines Unternehmens eine Schlüsselrolle ein. Um dieser Bedeutung und Wichtigkeit zu entsprechen, wird in diesem Wissensmanagement-Ansatz empfohlen, dass die Personalabteilung selbst für eine funktionierende Kommunikation innerhalb eines Unternehmens Verantwortung tragen sollte, da sie ja auch für die Einhaltung der institutionalisierten Hand- 
lungsabläufe verantwortlich zeichnet (vgl. das Kapitel "Die Rolle des Individuums"), dass Mitarbeiter die formalen Kommunikationsstrukturen des Unternehmens für ihre Aufgabenerledigungen verwenden. Geht man davon aus, dass die Mitarbeiter in ihrem Arbeitsbereich eingeschult wurden und genau wissen, wie und mit wem sie ihre Arbeiten erledigen sollen, könnte vor allem über die Geschwindigkeit der Aufgabenerledigung ein komparativer Vorteil gegenüber den Mitbewerbern geschaffen werden. Je besser Mitarbeiter koordiniert sind und miteinander kooperieren, desto schneller werden sie die Ziele ihres Aufgabenbereichs erfüllen. Institutionen bieten einen Stabilisierungseffekt und durch diese geschaffene Ordnung wird eine Struktur geschaffen, mit der Arbeiten zielgerecht und zügig abgeschlossen werden können.

Das Konzept, das auf ähnliche Weise operiert und in den vorliegenden Wissensmanagement-Ansatz einfließen soll, stellt das Konzept der Communities of Practice dar. Auch wenn die den Gemeinschaften zugrunde liegenden Annahmen in einer interpretativen Logik verortet werden müssen, so wurden Versuche gestartet, sie bewusst in das Unternehmensgeschehen zu integrieren Wenger/Snyder, 2000). Findet sich in solchen Gemeinschaften auf impliziter Ebene ein Grundkonsens darin, welche Richtlinien bestehen und welcher Handlungsspielraum vorhanden ist, sowie ein grundlegendes Verständnis von gewissen Rollenverteilungen (Wenger/Snyder, 2001), wird im Zuge dieses Wissensmanagement-Ansatzes der Versuch unternommen, ein derartiges informelles und selbstorganisatorisches Vorgehen auf einer expliziten, formalen organisationalen Ebene zu etablieren (zur Kritik an einem solchen Vorgehen vgl. Kapitel "Wissen managen - eine symbiotische Beziehung oder Widerspruch in sich?").

Sind solche Strukturen nicht in das Unternehmensgeschehen integriert, werden sich diese Kommunikationsnetzwerke in einem Unternehmen auf informeller Ebene bilden, die jedoch dann für ein Management nur mehr schwer, wenn überhaupt, zugänglich und steuerbar sind. Es soll betont werden, dass es in keinster Weise im Sinne der Unternehmensführung sein kann zu versuchen, informelle Netzwerke oder Gemeinschaften zu unterbinden oder zu leugnen jedoch sollte 
das Hauptaugenmerk auf die formalen Organisationsstrukturen gelegt werden, die sich im Einflussbereich des Managements darstellen und über formale Verträge regeln lassen.

Communities of Practice bilden einen ersten Ansatz, soziale organisationale Strukturen zu etablieren, im Rahmen derer Kommunikation auf individueller Basis die Rolle zugesprochen erhält, die notwendig ist, um Wissensteilung und generierung stattfinden zu lassen. Individuen lernen im Rahmen von Kommunikationsprozessen, Werte und Normen anderer zu akzeptieren und definieren selbst inre eigene Identität in den sozialen Praktiken, deren Teilnehmer sie sind. Dadurch wird ein Rahmengerüst an Spielregeln und aufgeteilten Rollen etabliert, was wiederum rekursiv auf die Handlungen selbst Einfluss übt. Der Zweck solcher Strukturen geht weit darüber hinaus, sich "nur" mit anderen auf inhaltlicher Ebene auszutauschen. Vielmehr erweitern Individuen im Rahmen dieser Interaktionsprozesse ihr Verständnis von unterschiedlichen sozialen Konstruktionen und Zugängen zur Welt.

Auf Basis einer gemeinsamen Zielorientierung wird ein offener Austausch über die jeweils subjektiven Konstruktionen der Wirklichkeit verfolgt und es werden Einsichten gewonnen, die ohne diesen Austausch nicht zugänglich wären. Die Gemeinschaft entwickelt eine gemeinsam etablierte Interpretation eines bestimmten Sachverhaltes, wobei die unterschiedlichsten Gesichtspunkte integriert werden. Dabei ist es nicht immer nötig oder wünschenswert, Bedeutungen völlig zu teilen, solange im Rahmen der Kommunikation die Teilnehmer an der sozialen Praktik wissen, wie sie entsprechend ihrer Rollen und Regeln auf die „Regeln des Spiels" zu reagieren haben (Pawlowsky/Bäumer, 1996, S. 226).

Dennoch darf nicht außer Acht gelassen werden, dass sich derartige Angleichungen einzelner Realitäten auch innerhalb von Communities sehr stark von den dort herrschenden Machtstrukturen abhängig zeichnen (vgl. Gherardi/Nicolini, 2001) und ein gemeinsames Verständnis der Wirklichkeit durch Verhandlungsprozesse entwickelt wird (vg! Wenger 1998). Und d [C]ommon sense is 
only commonsensical because it is sense held in common" (Wenger, 1998, S. 47). Dieser gemeinsame Konsens darüber, was nun wie zu beurteilen sei, birgt jedoch die Gefahr in sich, dass Mitglieder einer Community "labeled" bzw. „stigmatized" werden bzw. durch das "group-thinking" eine strenge Kontrolle ausgeübt wird; eine Art von Kontrolle, die sich sonst nur in nicht-hierarchischen Gruppen zwischen Freunden und Kollegen findet (Czarniawska, 1997, S. 56). Damit jedoch eine derartige Situation, in der negative Kontrolle und engstirniges Denken Gemeinschaften prägen, nicht eintritt, muss ein Kommunikationsklima bestehen, in dem eine gemeinsame Absicht, eine geteilte Vision und ein Verständnis davon, wie einer den anderen in seinen Leistungen ergänzen kann (Senge, 1993), vorhanden sein, was durch die Förderung von Communities of Practice erzielt werden kann.

Das Wissen, das im Rahmen der Interaktionen auf informeller Basis geteilt und neu entwickelt wird, wird eher zugänglich, als wenn man informelle Kommunikationsnetzwerke in einem Unternehmen missachtet und deren Bedeutung nicht richtig einschätzt.

Die Aufgabe des Managements bildet dabei die Unterstützung, Koordination und Motivation der einzelnen Mitarbeiter. Durch die Bildung von gemeinsamen Werten und Normen wird der Schwerpunkt auf die Organisationskultur gelegt und eine Wertebasis geschaffen, mit der sich Mitarbeiter eines Unternehmens identifizieren können. Grenzen innerhalb des Unternehmens werden durchbrochen und Wissensmanagement wird als Versuch gewertet, normative "Kontrolle" auszuüben. Somit umgeht man die Situation, dass "tacit knowledge" dem Management unzugänglich bleibt, und versucht dies, mittels einer aktiv geförderten Unternehmenskultur so gut wie möglich steuerbar zu machen (Alvesson/Karreman, 2001).

Implizites Wissen stellt sich zwar nicht unmittelbar als steuerbar dar. Es kann jedoch durch Zwischenschaltung von Personen und dem Aufbau einer fundierten Unternehmenskultur dem Management zugänglich gemacht werden Mittels einer $_{\text {Saran }}$ 
bewussten Förderung von Communities of Practice wird mittelbar ein Management von implizitem Wissen möglich.

Die Diskrepanz zwischen den formalen und informellen Austauschbeziehungen in einem Unternehmen schneidet einen Problembereich an, der eng mit einem Transfer von implizitem in explizites Wissen verknüpft ist. Implizites Wissen ist dasjenige Wissen, das potentiell auf eine organisationale Ebene transferiert werden kann; es ist an Personen gebunden und ein Schatz an individuellen Fähigkeiten und Fertigkeiten. Die kritische Frage für Unternehmen ist, wie weit es für das Unternehmen notwendig sein kann bzw. ist, dieses implizite Wissen auf eine organisationale Ebene zu bringen. Die Grenze, die hier gezogen werden muss, kann als der Punkt eines Wissensmanagement-Ansatzes festgehalten werden, der über Erfolg oder Misserfolg entscheidet. Gibt man sich der Illusion hin, dass man das gesamte implizite Wissen von Individuen für ein Unternehmen zugänglich machen muss, um einen Vorteil gegenüber seiner Konkurrenz generieren zu können, so wird die Natur des Menschen insofern verleugnet, als sie als Maschinen gesehen werden und soziale Aspekte keine bzw. mangelnde Berücksichtigung erfahren. Verneint man jedoch überhaupt die Notwendigkeit, implizites Wissen auf eine organisationale Ebene transferieren zu müssen, um Wissensmanagement betreiben zu können, so lässt man bewusst die Steuerungsmöglichkeiten des Managements außer Acht.

Ähnlich verhält es sich mit der Kontroverse zwischen formalen und informellen Austauschbeziehungen. Formale Regelungen und Verfahrensweisen strukturieren das Arbeitsgeschehen und bieten Unterstützungsfunktionen. Dennoch entstehen parallel dazu informelle Netzwerke und Kommunikationsstrukturen, die in Form von informellen Verträgen existieren. Auch hier entscheiden das Ausmaß und die Beziehung zwischen beiden Ebenen über den Erfolg oder Misserfolg eines Wissensmanagements. Erfüllt ein Unternehmen den definierten Unternehmenszweck und die Unternehmensziele und erledigen Mitarbeiter ihre Aufgaben, sollte für ein Unternehmen ein ausreichender Formalisierungsgrad erreicht sein. Parallel dazu sollten sich Mitarbeiter selbst entfalten, Kreativität,entwickeln 
und verbessern können, was sie unter anderem im Rahmen informeller Austausch- und Interaktionsprozesse erzielen werden. Dabei können die Wirkungen von Kommunikationsstrukturen und Regeln der Kommunikation als massiv konstatiert werden. Jenseits der Absichten und Wünsche der beteiligten Personen sorgen sie dafür, dass ein gemeinsamer Sinn entstehen kann oder verhindert wird, dass die Vergemeinschaftung von Erfahrung gelingt oder misslingt, dass sich ein Teamgeist bilden kann oder unterbunden bleibt (Willke, 2001). 


\subsection{Wissen verfeinern - Qualitätsmanagement}

Meine wichtigste Erfahrung als Manager ist die Erkenntnis, dass die Mitarbejter das wertvollste Gut eines Unternehmens sind und damit auch das wichtigste Erfolgskapital. Es sind nie Computer, Roboter, technische Einrichtungen, die zu einem Ziel führen, sondern immer Menschen, die Konzepte zustande bringen. Werner Niefer

Auch wenn in Unternehmen organisationale Strukturen integriert wurden, die ein effizientes Zusammenspiel von Mitarbeitern ermöglichen sollen, und diese die Kommunikationsstrukturen anlehnend an den institutionalisierten Handlungsabläufen eines Unternehmens verwenden, um ihre Arbeiten erledigt zu bekommen, bietet all dies keine Gewähr dafür, dass Unternehmen tatsächlich langfristig erfolgreich sind, ihre Nutzenfunktion optimal erfüllen oder einen komparativen Vorteil gegenüber ihren Mitbewerbern erzielen. Unternehmen werden sich zwar Situationen gegenübersehen, in denen sie mit einer solchen Organisationsstruktur, die das Organisationsproblem auf ein Minimum reduziert, besser als ihre Konkurrenz agieren können. Doch im Rahmen eines Wissensmanagement-Ansatzes wird nicht nur auf bestehende Vorteile und auf deren Beibehaltung Wert gelegt, sondern vielmehr wird einer dynamischen Perspektive Bedeutung geschenkt. Denn steigende Komplexität, Diskontinuität und Dynamik bezeichnen wesentliche Charakteristika der Unternehmensumwelt, und die Überlebensfähigkeit von Unternehmen hängt entscheidend davon $a b, o b$ sie in der Lage sind, sich permanent zu entwickeln und sich an Veränderungen der Umwelt anzupassen, sich diese zu Nutzen zu machen bzw. selbst zu intendieren.

Institutionalisierte Handlungsabläufe, die eine gewisse Allgemeingültigkeit an Wirklichkeitsauffassungen für die Organisationsmitglieder in Form intersubjektiv geteilter Wirklichkeitskonstruktionen beinhalten, also objektivierte Hypothesen der Organisationsmitglieder über organisationales Verhalten und deren Konsequenzen (Pawlowsky, 1992), können in statischer und dynamischer Form auftre- 
ten. Einerseits existieren diese statischen Routinen in Form von rigiden Handlungsprogrammen, die schwer veränderbar sind wie beispielsweise formalisierte Routineaufgaben, die die Funktion der Handlungsentlastung übernehmen und eine Orientierungshilfe bieten. Auf der anderen Seite stellen dynamische Routinen flexible Handlungsprogramme zur Verfügung, die im Zuge von Lern- und Entwicklungsprozessen verändert bzw. angepasst werden können, indem neue Handlungskompetenzen erworben und integriert werden.

Der Schwerpunkt eines Qualitätsmanagements wird auf den dynamischen Aspekt eines Wissensmanagements gelegt. Statische Handlungsabläufe werden so weit detailliert, dass eine Erfüllung der Unternehmensziele unter Berücksichtigung der Minimierung des Organisationsproblems gewährleistet werden kann. Die individuelle Gestaltung und Bearbeitung von Aufgabenstellungen bleibt den Mitarbeitern überlassen, die im Rahmen flexibler Handlungsprogramme frei agieren können. Auch an dieser Stelle kann nochmals gezeigt werden, dass der kritische Punkt eines Wissensmanagements sich darin zeigt, wo die Grenze zwischen formalen Regelungen und einem offenen und individuell gestaltbaren Handlungsspielraum gezogen wird. Bei Nonaka/Takeuchi (1995) wird dieser Sachverhalt unter "Autonomie" zusammengefasst; sie subsumieren darunter die Freiheit der Mitglieder einer Organisation innerhalb der gegebenen Rahmenbedingungen kreativ zu werden und Chancen zu nutzen.

Die Auseinandersetzung mit einem Wandel von Organisationsstrukturen sowie von Handlungsabläufen setzt an der gestiegenen Bedeutung von Anpassungsund Entwicklungsprozessen in und von Organisationen an. Veränderungen der Umwelt werden immer weniger vorhersehbar und prognostizierbar.

Bereits Nonaka/Takeuchi (1995) stellten als zentrales Charakteristikum erfolgreicher Firmen die Erkenntnis in den Raum, dass neues Wissen nicht einfach aus der Verarbeitung objektiver Informationen entsteht, sondern dass dieser Vorgang ebenso von den stillschweigenden und oft höchst subjektiven Einsichten, Eingebungen und Mutmaßungen der Einzelnen abhängt, diesess zu präfen und im Sin- 
ne des jeweiligen Unternehmens zu nutzen. Als Hebel dienen dabei persönliches Engagement und die Bereitschaft aller, sich mit dem Unternehmen und seinem Unternehmenszweck zu identifizieren.

Firmenspezifische Ressourcen und Kompetenzen stellen einen kritischen Faktor dar, Unternehmen zu ermöglichen, einen Mehrwert beim Kunden zu erzielen. Analysen in Richtung von Fertigkeiten und Kapazitäten eines Unternehmens sind mittlerweile von höherem strategischen Wert als eine detaillierte Analyse des im Wettbewerb stehenden Umfelds (Edmondson/Moingeon, 1996). Die Ausarbeitung von strategischen Vorteilen und das Aufweisen von Unterschieden zu der Konkurrenz erweisen sich jedoch als immer schwieriger. Bezugnehmend auf den ressourcenbasierten Ansatz der Theorie der Unternehmensführung (geprägt von beispielsweise Penrose, 1959; Peteraf, 1993) kann ein Unternehmen nur dann einen komparativen Vorteil geltend machen, wenn die am Markt angebotenen Leistungen "valuable", „scarce", „inimitable" und "non-substitutable" sind. Diesen Kriterien auch in der Praxis zu genügen, scheint in der Regel ein erfolgloses Unterfangen zu sein und vor allem die letzten drei werden durch die stetigen Weiterentwicklungen am technologischen Sektor kaum noch erfüllt. Dennoch behaupten sich einige Unternehmen besser als andere und auch wenn viele exogene wie endogene Faktoren dazu beitragen, soll dem Kriterium "valuable" eine besondere Beachtung geschenkt werden.

Hier werden Leistungen zusammengefasst, mit denen einerseits Chancen aus der Umwelt wahrgenommen und genutzt und andererseits Gefahren und Bedrohungen für das Unternehmen neutralisiert und abgewandt werden (Barney, 1991). Als "valuable" können auch lediglich solche Leistungen klassifiziert werden, die den Anforderungen eines Kunden entsprechen und einen Mehrwert für diesen darstellen. Die Gewährleistung einer erwünschten Qualität des betrieblichen Leistungserstellungsprozesses ist ein bedeutendes unternehmerisches Anliegen. Qualitätsrelevante Kriterien können sich auf Eigenschaften der angebotenen Dienstleistungen bzw. Produkte beziehen, sollten jedoch von einer wissens- 
managementtheoretischen Perspektive aus auch um Kriterien, die die Mitarbeiter und die Organisation eines Unternehmens betreffen, erweitert werden.

Qualität der betrieblichen Leistungserstellung und Leistungsverwertung stehen beispielsweise in den Konzepten des Total Quality Management, der Balanced Scorecard oder des EFQM-Modells im Mittelpunkt. Hierbei wird ein langfristiges, integriertes Unternehmenskonzept in einem Unternehmen etabliert, das die Qualität von Produkten oder Dienstleistungen entlang einer Wertschöpfungskette durch Mitwirkung aller Mitarbeiter zu günstigen Kosten kontinuierlich gewährleisten und verbessern soll, um eine optimale Bedürfnisbefriedigung der Leistungsabnehmer zu ermöglichen. Die Mitarbeiter eines Unternehmens werden im Rahmen eines Qualitätsmanagements integriert. Im Zuge eines EFQM-Modells wird beispielsweise der Umgang einer Organisation mit inren Mitarbeitern bewertet, wobei näher analysiert wird, wie ein Unternehmen das gesamte Potential seiner Mitarbeiter freisetzt, um ihre Geschäftstätigkeit ständig zu verbessern. Hierbei wird berücksichtigt, wie Unternehmen Mitarbeiterressourcen zukünftig planen und verbessern und die Kompetenzen und Fähigkeiten der Mitarbeiter bei Personalplanung, -auswahl und -entwicklung erhalten und weiterentwickelt werden, wie Mitarbeiter und Teams Ziele vereinbaren und ihre Leistungen ständig überprüfen, wie die Beteiligung aller Mitarbeiter am Prozess der ständigen Verbesserung gefördert wird bzw. wie diese autorisiert werden, selbst zu handeln und schlussendlich wie eine wirksame Kommunikation innerhalb eines Unternehmens erzielt wird (v. Eckardstein, 1999). Auch das Konzept der Balanced Scorecard (Kaplan/Norton, 1996) überwindet den Fokus auf Bestehendes und konzentriert sich auf eine qualitative Weiterentwicklung in sämtlichen Unternehmensbereichen. Nur wenn Unternehmen neue, erfolgreiche Produkte bzw. einzigartige Dienstleistungen anbieten, auf motivierte und gut eingeschulte Mitarbeiter zurückgreifen können und neue Technologien anwenden, werden sie eine Möglichkeit haben, die Nutzenfunktion ihres Unternehmens zu optimieren (vgl. Kaplan/Norton, 1996).

Den Ausgangspunkt für jegliche unternehmerische Aktivitäten, unabhängig davon, ob es sich um routinisierte Abläufe oder um die Durchführung spezieller 
Prozesse wie beispielsweise von Optimierungsprozessen oder dergleichen handelt, bildet das Individuum rekurrierend auf die Annahmen des der Theorie der Institutionenökonomie zugrunde liegenden methodologischen Individualismus. So soll auch an dieser Stelle der einzelne Mitarbeiter in den Vordergrund rücken.

Individuen als Initiatoren jeglicher Verbesserungs- und Innovationsprozesse werden sich vor allem dann frei entfalten können, wenn eine stabile Organisation zugrunde liegt. Etablierte Routinen bilden dabei die Ausgangskomponenten zur Entwicklung neuer Handlungskompetenzen. Ein Innovationsprozess gilt als eine Such-, Bewertungs-, Auswahl- und Routinisierungsprozedur, im Rahmen derer es neue Handlungsprogramme und Problemlösungswege zu erkennen, auszuwählen und in innovativen Routinen zu institutionalisieren gilt, sodass sie sich mit den bereits etablierten Verhaltensmustern ergänzen und "new combinations" (Nelson/Winter, 1982) an Handlungskompetenzen bilden.

Überlässt man als Unternehmen solche innovativen Prozesse nicht einem kreativen Chaos, sondern möchte man einen Nährboden innerhalb eines Unternehmens schaffen, wo neue Ideen bzgl. der Weiterentwicklung von Produkten oder Dienstleistungen entstehen können, wird dies über die bereits erwähnte Qualitätsabteilung erfolgen (vgl. Kapitel „Organisation - Ordnung und Struktur verleihen"). Diese würde für die Gewährleistung der unternehmerischen Qualität verantwortlich zeichnen und würde einerseits die am Markt angebotenen Produkte bzw. Dienstleistungen kontrollieren und bei Abweichungen eine Korrektur verlangen.

Andererseits würde es zu ihrem Aufgabenbereich zählen, in enger Zusammenarbeit mit der Personalabteilung Mitarbeiter weiterzubilden, sodass stetig eine Verbesserung ihrer Fertigkeiten und Fähigkeiten gewährleistet ist (vgl. die "Lern- und Entwicklungsperspektive" von Kaplan/Norton, 1996).

In enger Kooperation mit der Personalabteilung, die für die Einhaltung von institutionalisierten Handlungsabläufen zuständig zeichnet kann bei Nichtwissen von 
Akteuren in Form von betrieblicher Weiterbildung eine Ausgangsbasis geschaffen werden, sodass Mitglieder eines Unternehmens wissen, wie sie die an sie gestellten Arbeitsanforderungen erfüllen sollen. Ein Kompetenzproblem kann auf diese Weise überwunden werden.

Betriebliche Weiterbildung (jener Teil der beruflichen Weiterbildung, der vom Unternehmen durchgeführt bzw. veranlasst wird (Pawlowsky/Bäumer, 1996, S. 8)) kann jedoch auch nicht willkürlich durchgeführt werden, sondern muss sowohl Qualifikationsanforderungen erfüllen und Strategien umsetzen helfen, gleichzeitig aber auch strategiegestaltend wirksam sein. So kann erfolgsrelevantes Wissen identifiziert und entwickelt sowie dem Unternehmen verfügbar gemacht werden. Als primäre Aufgabe sollte dafür Sorge getragen werden, dass nicht lediglich eine Bereitstellung von anforderungsgerechten Qualifikationen erfolgt (Erfüllungsfunktion), sondern dass die Schaffung von Lern- und Entwicklungsmöglichkeiten des Unternehmens auf der Grundlage von Wissen ermöglicht wird (Gestaltungsfunktion) (Pawlowsky/Bäumer, 1996). Es sollten Maßnahmen gesetzt werden, die „systematisch, positions- und laufbahnorientiert eine Verbesserung der Qualifikationen der Mitarbeiter zum Gegenstand haben mit der Zwecksetzung, die Zielverwirklichung der Mitarbeiter und des Unternehmens zu fördern" (Conradi, 1983, S. 3).

Hierbei wird es erforderlich, dass die Umsetzung von Qualitätsmanagement die jeweilige Personalstrategie eines Unternehmens als eigenständige Komponente in die strategische Unternehmensplanung mit einbezieht und das gesamte Unternehmen umspannt. Auch wenn es eine für Qualität verantwortliche Stelle gibt, so bildet Qualitätsmanagement eine unternehmerische Querschnittsfunktion. Das bedeutet, dass die Erfüllung der im Unternehmen bestimmten Qualitätskriterien als Aufgabe bzw. Pflicht von jedem einzelnen Mitarbeiter getragen bzw. wahrgenommen werden sollte. Bei Auftreten von Abweichungen, sei es bezogen auf die erbrachten Produkte oder Dienstleistungen oder aber auf die Handlungsweisen der Mitarbeiter, die den in einem Unternehmen institutionalisierten Verfahrens- 
weisen zuwider laufen, soll eine Qualitätsabteilung auf Strukturen und Mechanismen zurückgreifen können, um diese zu beheben.

Investiert man in Individuen, so sollte das Unternehmen bereits nach kurzer Zeit eine Rendite davon erhalten. Auch das Individuum selbst sollte dies für seine eigene Nutzenfunktion erwarten können (vgl. beispielsweise Williamson, 1990). Nur auf diese Weise wird ein Vertrag zwischen beiden Parteien langfristig und erfolgsversprechend sein. „Zum Überleben von Organisationen ist es notwendig, dass Menschen sich entscheiden, in die Organisation einzutreten, diese Mitgliedschaft zumindest über einen gewissen Zeitraum aufrechtzuerhalten, die ihnen übertragenen Aufgaben in einer ungefähr vorhersagbaren Weise auszuführen und darüber hinaus auch kreativ, spontan und innovativ zu handeln" (Mayerhofer, 2002, S. 258).

Der Wert eines Mitarbeiters für ein Unternehmen kann durch ständige Aus- und Weiterbildung gesichert sowie gesteigert werden (North, 2002), was wiederum als Ausgangspunkt dafür gilt, dass Mitarbeiter innovative Prozesse initiieren sowie neue Ideen, Konzepte oder Verbesserungsvorschläge entwickeln und unterbreiten, sodass ein institutionalisierter Handlungsspielraum inklusive der Handlungsabläufe einer Weiter- und Fortentwicklung unterliegt. 


\subsection{Technologische Untermauerung}

Wissend ist, wer weiB, wo er findet, was er noch nicht weiB.

Georg Simmel

Als Fundament dieses Wissensmanagement-Ansatzes werden die organisationaIen Strukturen eines Unternehmens betrachtet. So spielen institutionalisierte Handlungsabläufe und Wertvorstellungen sowie Kommunikationsstrukturen eine bedeutende Rolle. Neben dem Beitrag von Organisationsstrukturen, institutionalisierten Handlungsabläufen sowie Kommunikationsstrukturen und einer wissensmanagement-orientierten Unternehmenskultur betrifft die Gestaltung der Informations- und Kommunikationstechnologien ein weiteres Bedingungs- und Unterstützungsfeld für Wissensmanagement. „Die Infrastruktur ist das Mittel, durch das eine Organisation die Ressourcen zur Verfügung stellt, die den Menschen bei ihrer Arbeit helfen soll" (Senge, 1996, S. 35). Konzepte, die ausschließlich auf diesen Teilbereich des Wissensmanagements eingehen und eine humanorientierte Sichtweise nicht erkennen lassen (vgl. dazu beispielsweise Wittman, 1979; Ropohl, 1979; Heinrich, 1996; Martiny/Klotz, 1990), wurden in dieser Arbeit nicht vorgestellt und auf diese Ansätze wird in Folge auch nicht rekurriert werden.

Mit Informationstechnologien wird unter anderem das Ziel verfolgt, die Reichweite und Geschwindigkeit beim Wissenstransfer zu erhöhen, was als ein Schlüsselpunkt für einen komparativen Vorteil von Untenehmen gegenüber ihren Mitwebern festgehalten wurde (vgl. das Kapitel "Kommunikation - der Schlüsselfaktor") Gegenstand dieses Prozesses kann jedoch lediglich explizites Wissen sein, das sich mit Hilfe von Technologien dokumentieren, speichern und verwalten lässt. Erfolgt ein Wissenstransfer auf informeller Basis, wie es in den Communities of Practice der Fall ist, so werden sich die Inhalte dieser Austauschprozesse kaum erfassen lassen, da sie sich einer formalen Strukturierung entziehen. Die Austauschprozesse selbst können wiederum sehr wohl durch Medien aus der Informationstechnologie unterstützt werden (beispielsweise über E-Mail, ein Unternehmensintranet, Unternehmensplattformen und dergleichen). 
Das Maß an Kodifizierung und Strukturierung von explizitem Wissen darf jedoch die Möglichkeiten der Informationstechnologien nicht bis ins Endlose ausreizen. Verwirklichbar ist mit den neuen Medien viel, jedoch müssen die Kriterien der Nützlichkeit und Verwendbarkeit für das jeweilige Unternehmen definiert und eingehalten werden. Denn es geht nicht darum, den IT-Einsatz zur Unterstützung von Wissensmanagement zu maximieren, sondern eine angemessene, zielorientierte IT-Verwendung zu gestalten (vgl. Probst et al., 1999).

Die Aufgabenfelder der Wissenssuche, -(ver)teilung und -bewahrung werden von Informationstechnologien geprägt. Verschiedene Instrumente stehen zur Verfügung, um diese einzelnen Prozesse zu unterstützen und zu beschleunigen. Eine erfolgreiche Nutzung wird sich jedoch nur dann ergeben, wenn eine dafür förderliche Unternehmenskultur besteht, wenn Austauschprozesse gelebt werden und wenn eine offene Kommunikation zwischen den Mitarbeitern vorhanden ist (vgl. North, 2002).

Wird der Gesamtkontext des jeweiligen Unternehmens gesehen und kann auf einer im Unternehmen vorhandenen Wissensorientierung aufgebaut werden, so sollte ein Mitarbeiter Zugriff auf ein unternehmensweites, wissensorientiertes, integratives Netzwerk erhalten. Hierfür gibt es einerseits ein Instrumentarium, das den Prozess der Wissenssuche unterstützt, beispielsweise Gelbe Seiten oder digitale Wissenslandkarten (Wissensspinnen). Andere WissensmanagementTools greifen weiter und fungieren als Basisinfrastruktur eines ganzheitlichen Wissensmanagements. Über sog. "Intranets" kann das obig erwähnte unternehmensweite Netzwerk verwirklicht werden (genaue Ausführungen, was diese Basistechnologie beinhaltet, finden sich in jedem aktuellen einschlägigen Fachbuch, vgl. beispielsweise das Lexikon der Wirtschaftsinformatik von Mertens, 1997). Erweitert werden Intranets durch Groupware-Systeme, die in unterschiedlicher Form und unterschiedlichem Ausmaß die Kommunikation, Koordination und Kooperation bei der gemeinsamen Aufgabenbearbeitung durch Gruppen unterstützen (vgl. detaillierte Ausführungen bei beispielsweise Thiesse, 1999, bzw. in aktuellen einschlägigen Fachbüchern; Probst et al 1997) Vor allem der Prozess 
der Wissensverteilung kann mit solchen Systemen beschleunigt und unterstützt werden. Eine für ein Unternehmen förderliche informationstechnologische Realisierung von Wissensmanagement lässt sich erreichen, wenn Intranets mit Groupware-Systemen zu integrierten Basisplattformen verbunden werden.

Da die neuen Informations- und Kommunikationstechnologien für Organisationen Infrastrukturen bereitstellen, die einen erheblichen Anteil einer Wissensorganisation ausmachen (vgl. Roehl, 2000), werden hohe Erwartungen in sie gesetzt. Eine informationstechnologische Infrastruktur ist als eine Bedingung für Wissensmanagement und als der technologische, Wissen organisierende Rahmen der Organisation zu sehen. Informations- und Kommunikationstechnologien werden nicht lediglich an einer Stelle eines Unternehmens verwendet, sondern umspannen netzwerkartig das gesamte Unternehmen.

Diese können für verschiedene Zwecke in einem Unternehmen eingesetzt werden, was bei Zuboff (1988) unter "informating" zusammengefasst wurde: Einerseits kann es Mitarbeiter eines Unternehmens dabei unterstützen, Informationen für andere zugänglich zu machen ("informating") und andererseits kann es als Instrumentarium verwendet werden, das Verhalten der Mitarbeiter zu lenken und zu steuern.

Eine statische Aufnahme der internen Beziehungsstrukturen in einem Unternehmen könnte mit Hilfe von Informationstechnologien nachgezeichnet werden. So würde die vertragliche Gliederung eines Unternehmens mit seinen Entscheidungs- und Machtstrukturen digital festgehalten werden und die einzelnen Aufgabenbereiche mitsamt den damit einher gehenden Verantwortlichkeiten, Rechten und Pflichten darstellen.

Die Koordination der Mitarbeiter kann durch eine informationstechnologische Untermauerung unterstützt werden. So können institutionalisierte Handlungsabläufe als "best practices" ebenfalls digital erfasst werden, wodurch parallel dazu die formalen Kommunikationsstrukturen eines Unternehmens gesichert werden 
können. Denn mit den standardisierten Routinen sollten die Informationen darüber, wer mit wem in welchem Ausmaß an einer speziellen Aufgabe arbeitet, abgespeichert werden. Der Zweck dieser Handlungsabläufe sollte angegeben werden, um zu gewährleisten, dass sich Mitarbeiter als Teil eines Unternehmensganzen verstehen. Diese Fülle an Informationen wird jedoch lediglich dann ihre Zweckmäßigkeit erfüllen, wenn sie nicht an einer Stelle des Unternehmens versickert, sondern sämtlichen Mitarbeitern zugänglich gemacht wird.

Die Modellierung von Kommunikationsstrukturen und Arbeitsabläufen in elektronischen Infrastrukturen zementiert diese gleichsam als organisationale Routinen, denn sie können immer nur zeitpunktbezogene und statische Lösungswege wiedergeben. Für zukünftige Entwicklungen der Organisation und der Komplexität ihrer Problemstellungen entfaltet dies eine erhebliche Bedeutung (Probst/ Raub/ Romhardt, 1997).

Mitarbeiter werden somit nicht nur vertraglich in ein Unternehmensganzes eingeordnet, sondern erhalten auch mit Hilfe von Informationstechnologien eine Orientierungshilfe. So können sie von ihrem eigenen PC aus ihre Position im Unternehmen orten und die zu ihrem Aufgabenbereich zugehörigen Handlungsabläufe digital abrufen. Hierfür bieten die Informationstechnologien einige Applikationen an, wie beispielsweise ein Intranet, das ein unternehmensweites Netzwerk bezeichnet und als technologische Systemvoraussetzung über eine reine Informationsbereitstellung hinaus zunehmend Bedeutung für die Unterstützung abteilungsübergreifender Teamarbeit im Hinblick auf Kommunikation, Koordination und Kooperation hat (auch Groupware-Systeme erfüllen diese Funktion).

Mitarbeitern, die über keinen eigenen Zugang zu diesem Intranet verfügen, sollte eine Arbeitsstelle zugänglich sein, von der aus sie in das Intranet einsteigen, um die dort enthaltenen Informationen zu erhalten. Abhängig von den intranetbasierten Anwendungen wird eine organisationsweite interaktive Verteilung von Informationen unterstützt, indem beispielsweise Datenbanken angeboten werden. Für eine kollektive Aufgabenbearbeitung können Workflow-Management- 
Systeme bis hin zu interaktiv zugreifbaren Zeit- und Projektplänen implementiert werden. Im Zuge dieser Anwendungen kann es zu einer konkreten Definition von individuellen Zielquoten kommen, um zu gewährleisten, dass Unternehmensziele erreicht werden, was folglich wiederum sicherstellen kann, dass sich Mitarbeiter mit ihren Tätigkeiten in einem Unternehmensganzen nicht verlieren. Auf ähnliche Weise wurde ein solches Vorgehen bereits bei der Entwicklung einer Balanced Scorecard (Kaplan/Norton, 1996) aufgegriffen.

Beim Einstieg in ein Unternehmen zeichnet die Personalabteilung verantwortlich, neue Mitarbeiter auf dieses digitale Abbild der Unternehmensabläufe einzuschulen, sodass sich diese leichter in das unternehmerische Gesamtgefüge integrieren können (vgl. das Kapitel "Kommunikation - der Schlüsselfaktor").

Auch sollte eine informationstechnologische Basisinfrastruktur Applikationen anbieten, die einen informellen Austausch zwischen Mitarbeitern unterstützen. Als Beispiele können Dienste wie E-Mails oder Chat-Rooms genannt werden. Sämtliche weiteren Möglichkeiten, die sich einem Unternehmen bieten, um eine angenehme Unternehmensatmosphäre zu schaffen, wo Mitarbeiter gerne interagieren und sich austauschen, werden hier nicht explizit behandelt, könnten jedoch Ausflüge, Firmenfeiern oder eine Firmenkantine umfassen.

Dieses Abbild an statischen Beziehungs- und Kommunikationsstrukturen sowie an institutionalisierten Handlungsabläufen eines Unternehmens weist jedoch noch kein Wissensmanagement aus. Auch der dynamischen Perspektive von Wissensmanagement muss im Rahmen von Informationstechnologien Beachtung geschenkt werden. So sollten diese über Applikationen verfügen, die es Mitarbeitern ermöglichen, standardisierte Handlungsabläufe zu verändern, wodurch ein Handlungsspielraum geschaffen wird, in dem Kreativität und Individualität Platz haben. Verlaufen organisationale Strukturen und vertragliche Vereinbarungen zu starr, wird die Rolle der Mitarbeiter auf "Maschinen", deren Steuerung vollkommen der Unternehmensführung unterliegt, reduziert, was sich jedoch für ein Wissensmanagement aufgrund einer inkompatibilität mit den dieser Arbeit zugrunde 
gelegten Annahmen der Theorie der Neuen Institutionenökonomie als nicht tragbar darstellt.

Um eine Weiterentwicklung des Unternehmens zu ermöglichen, bedarf es einer Basistechnologie, mittels derer Mitarbeiter ihre Ideen bzw. Verbesserungs- oder Ånderungsvorschläge bekannt geben können, was in Form eines digitalen Vorschlagswesen gewährleistet werden könnte. Da die Qualitätsabteilung für die Entwicklungsfähigkeit des Unternehmens sowie der Mitarbeiter Verantwortung trägt, sollte sie Adressat für Veränderungen und Neuerungen sein, wodurch die mangelnde Flexibilität und Dynamik von informationstechnologischen Anwendungen umgangen werden sollten. Beispielsweise würden Diskussionsforen oder Blackboards eine digitale Hilfestellung bieten. Die Qualitätsabteilung sammelt diese Vorschläge, wertet sie z.B. mit Hilfe von Szenarientechniken aus, arbeitet sie gegebenenfalls in routinisierte Handlungsabläufe ein, die dann den Mitarbeitern als neue Standardverfahren zur Verfügung stehen und im Laufe ausreichender Nutzung und Integration institutionalisiert werden könnten.

Damit eine technologische Untermauerung des Unternehmensgeschehens nicht in einer Datenfülle resultiert, die einerseits nicht genutzt wird und andererseits lediglich in einem Chaos endet, werden die Rechte jedes einzelnen Mitarbeiters im System genau festgesetzt. Abhängig von den vertragstheoretischen Bestimmungen der Mitarbeiter sind diese unterschiedlich in einem Unternehmen positioniert und mit verschiedensten Rechten und Pflichten ausgestattet, sodass sich dies in einem technologischen System widerspiegeln sollte.

Eine Analyse dessen, welche Ausstattung an spezifischer Hard- und Software in einem Unternehmen Wissensmanagement auf optimalem Weg unterstützen könnte, wird als situationsabhängig eingestuft und an dieser Stelle nicht weiter ausgeführt.

Mittels einer informationstechnischen Basisinfrastruktur innerhalb eines Unternehmens sollen Unternehmen in der Überwindung des Organisationsproblems 
unterstützt werden, da sie Mitarbeitern zur Verfügung steht, um die Austauschprozesse innerhalb eines Unternehmens zu vereinfachen und zu beschleunigen. Es kann zu einer besseren Koordination von Mitarbeitern kommen. Da auch Applikationen angeboten werden sollen, die nicht unmittelbar formale Beziehungsstrukturen widerspiegeln, sondern den informellen Austausch zwischen Mitarbeitern fördern sollen, wird versucht auch das Motivationsproblem von Akteuren zu verringern.

Im Zuge dieses Wissensmanagement-Ansatzes wird zwischen Technologien, die die Produktion oder das Geben von Dienstleistungen eines Unternehmens unterstützen, und denjenigen, die die Organisation eines Unternehmens betreffen, unterschieden. So werden letztere als technologischer Ausgangspunkt für Wissensmanagement gewertet. Unabhängig davon, was in einem Unternehmen schlussendlich am Markt vertrieben wird, soll sich Wissensmanagement in diesem Ansatz an der bestmöglichen internen Organisation von Unternehmensaktivitäten orientieren. 


\subsection{Abschließende Betrachtung}

Wissensmanagement stellt einen gemeinhin akzeptablen Lösungsweg dar, um Wettbewerbsnachteile eines Unternehmens zu kompensieren bzw. zum Positiven zu wenden. Mit der theoretischen Fundierung durch die Theorie der Neuen Institutionenökonomie wird eine Ausgangssituation geschaffen, auf der aufbauend Wissensmanagement in einem Unternehmen Eingang finden kann.

Als ausschlaggebend dafür, ob sich Wissensmanagement für ein Unternehmen als erfolgreich oder nicht erfolgreich darstellt, wurde einerseits in der Grenzziehung zwischen implizitem und explizitem Wissen sowie in der Definierung von handlungsrelevantem Wissen gewertet. Um implizites Wissen auf eine organisationale Ebene zu transferieren, bedarf es einer Artikulation durch Individuen, die als Quelle von Wissen konstatiert wurden.

Der Formalisierungsgrad eines Unternehmens zeichnet weiters für einen Erfolg oder Misserfolg von Wissensmanagement verantwortlich. Wissen sollte soweit externalisiert und institutionalisiert in Handlungsabläufen, Routinen, Normen und Werten eines Unternehmens festgehalten werden, dass Mitarbeiter die in ihren Verträgen zusammengefassten Arbeitsbereiche erledigen können, sodass die definierten Unternehmensziele und der Unternehmenszweck erfüllt werden können.

Institutionen üben in diesem Zusammenhang einen stabilisierenden Einfluss aus. Dieser Handlungsspielraum sollte klar abgegrenzt sein und für sämtliche Mitarbeiter eines Unternehmens zur Verfügung stehen und in Verwendung geraten.

Für ein Unternehmen ist der Bereich zwischen implizitem und explizitem Wissen ein Terrain, wo Sensibilität und Fingerspitzengefühl gefordert werden. Es sollte einerseits zu keiner Einschränkung der Persönlichkeiten von Individuen kommen, indem das Wissen von Mitarbeitern für das jeweilige Unternehmen und dessen Zielerreichung bis ins letzte Detail externalisiert werden. Andererseits sollte eine 
Überformalisierung verhindert werden, damit ein reibungsloses Arbeiten ermöglicht wird.

Das Wissen, das im Zuge eines Wissensmanagements adressiert werden sollte, stellt das handlungsleitende Wissen eines Unternehmens dar. Handlungsleitend wird Wissen, wenn es in den institutionalisierten Spielregeln verankert ist. Der Prozess der Institutionalisierung und somit des Transfers von implizitem in organisationales Wissen erfolgt mit den Informationen, die sich für ein Unternehmen als richtungweisend zeigen, sowie durch die Informationen, die die in einem Unternehmen durchgeführten Tätigkeiten gewährleisten können. An dieser Stelle wird der normative und präskriptive Charakter von Institutionen hervorgehoben.

Artikuliertes Wissen, das nicht unmittelbar zu Produktionsergebnissen führt bzw. das Erreichen der Unternehmensziele nicht offensichtlich unterstützt, sich jedoch für die zusammenarbeitenden Mitarbeiter als sehr wertvoll darstellt, benötigt ebenfalls eine Legitimations- und Existenzbasis. Ein Unternehmen zeichnet verantwortlich dafür, eine Atmosphäre zu schaffen, in der sich Mitarbeiter in einer Form entwickeln und entfalten können, dass sie ihr Wissen auf freiwilliger Basis in ein Unternehmen einbringen und weitergeben und ein Wissensaustausch gewünscht und dadurch "gelebt" wird. Natürlich lassen sich auch Anreizmechanismen schaffen, die eine Kooperation seitens der Mitarbeiter gewährleisten helfen, um die zugrunde liegenden Verhaltensannahmen wie Opportunismus, begrenzte Rationalität etc. zu überwinden.

Der Schwerpunkt im Rahmen des elaborierten Wissensmanagement-Ansatzes wird auf die formalen Kommunikationsstrukturen und institutionalisierten Handlungsabläufe gelegt, da dadurch das Organisationsproblem überwunden werden soll. Haben die einzelnen Akteure eines Unternehmens Kenntnis davon, mit welchen Personen in einem Unternehmen an welchen Aufgabengebieten zusammengearbeitet wird, welche Ansprechpartner in welchen Angelegenheiten zur Verfügung stehen, für welche Personen im Unternehmen sie selbst ein Informationslieferant sind, können Mitarbeiter ein stabiler Ausgangspunkt, werden, von 
dem ein ziel- und zweckorientiertes Arbeiten möglich wird, vorausgesetzt, es stehen in einem Unternehmen institutionalisierte Handlungsabläufe zur Verfügung und Mitarbeiter können sich in einer klaren Abgrenzung ihres Arbeitsbereichs, ihrer Rechte und Pflichten mit dem nötigen Freiraum bewegen.

In der in dieser Arbeit gezeichneten organisatorischen Ausgestaltung von Unternehmens- und Kommunikationsstrukturen wird auf normative Weise eine mögliche ideale Vorstellung eines Zusammenspiels in Unternehmen dargestellt. Eine derartige Skizzierung kann jedoch nicht abgehoben von der Realität bestehen, sondern muss in Beziehung zu den realen Gegebenheiten in Unternehmen gesetzt werden.

Somit wird sich die tatsächliche Strukturierung einer Organisation spezifisch je Unternehmen unterscheiden. Was unter "realen Gegebenheiten" verstanden wird, muss an dieser Stelle unbeantwortet bleiben, denn jedes Unternehmen ist eingebettet in seiner eigenen Konstruktion der Wirklichkeit, die Personen über eine Realität übereinstimmen lässt.

Wissensmanagement zeichnet sich jedoch nicht ausschließlich dadurch aus, dass eine optimale Aufzeichnung der internen Organisationsstrukturen und Handlungsabläufe angestrebt wird, mit der Mitarbeiter eine Orientierungs- und Stabilitätshilfe erhalten, sondern vor allem durch das unternehmerische Potential, eine stetige Fort- und Weiterentwicklung des Unternehmens zu gewährleisten. Ein Initiator und Verantwortungsträger für derartige Prozesse sollte in einem Unternehmen integriert sein, wodurch strukturell diese Intention der dynamischen Zukunftsausrichtung verankert wird. Es wurde hierfür eine eigene Abteilung, die Qualitätsabteilung, vorgeschlagen, wobei die Ausführungen zu dieser Thematik altbewährten Managementkonzepten, die den Faktor Qualität in den Mittelpunkt stellen, eine entsprechende Bedeutung zuschreiben.

Das Zusammenspiel der Personal- mit der Qualitätsabteilung, die klare vertragliche Ausgestaltung der Beziehungsstrukturep eines Unternehmens yorhandene 
institutionalisierte Handlungsabläufe, Unternehmensziele, ein Unternehmenszweck sowie motivierte und effiziente Mitarbeiter stellen eine Voraussetzung für Wissensmanagement dar.

Werden die unternehmerischen Prozesse unter Verwendung von institutionalisierten Handlungsabläufen mit einer strukturellen Hervorhebung der Personalund Qualitätsabteilung sowie einer stetigen Verbesserung der Mitarbeiter und internen Prozesse durch einen fortwährenden Wissenstransfer von implizitem in organisationales Wissen durchlaufen, wodurch der institutionalisierte Handlungsspielraum ebenfalls einem Wandel unterliegt, kann unter Berücksichtung der in dieser Arbeit zugrunde gelegten Annahmen davon gesprochen werden, dass in einem Unternehmen Wissensmanagement durchgeführt wird. 


\section{Weiterführende Gestaltungsoptionen}

Organisationstheoretisch wurde dieser Arbeit die Theorie der Neuen Institutionenökonomie zugrunde gelegt, auf der aufbauend die Organisationsstruktur eines Unternehmens unter Berücksichtung von wissensmanagementtheoretischen Aspekten in einer Form gestaltet wurde, sodass die Durchführung eines erfolgreichen Wissensmanagements vor dem Hintergrund der Überwindung des Organisationsproblems gewährleistet wird.

Organisationsstrukturen, die im Sinne von wissensbasierten Ansätzen ein Unternehmen unterstützen, Wissen zu managen, sollten ihren Fokus auf die institutionalisierten Handlungsabläufe sowie auf das Individuum legen. Die Bedeutung einer Personal- und Qualitätsabteilung wurde hervorgehoben. Sie zeichnen für eine optimale Integration und Motivation der Mitarbeiter eines Unternehmens verantwortlich. Diese Ausgangsbasis sollte in jedem Unternehmen, das Wissensmanagement betreiben möchte, geschaffen werden. Somit stellen sich die in dieser Arbeit dargestellten Organisationsstrukturen als unabhängig vom unternehmerischen Umfeld sowie von der Unternehmensgröße dar.

Diese beiden Komponenten müssen zwar Berücksichtigung bei der konkreten Ausgestaltung der Organisation eines Unternehmens erfahren, dies sollte jedoch parallel zu den strukturellen Erneuerungen geschehen, damit Wissensmanagement auf die in dieser Arbeit gezeichnete Weise durchgeführt werden kann. Wissensmanagement ist nicht Großbetrieben vorbehalten bzw. Unternehmen, die sich in ganz speziellen Branchen befinden. Vielmehr stellt sich ein erfolgreiches Wissensmanagement in der einen oder anderen ausgeprägten Form für jedes Unternehmen als wettbewerbskritisch dar. 
Eine Einführung von Wissensmanagement in ein beliebiges Unternehmen wurde in dieser Arbeit nicht behandelt, könnte jedoch ein Gegenstand weiterer Analysen sein. Durch die Einfachheit des in dieser Arbeit dargestellten Wissensmanagement-Ansatzes, der vielfach Modelle, die in Unternehmen bereits Anwendung finden, gezielt einzuarbeiten versucht, sollte auch ein Implementierungsprozess einfach gestaltet werden. Auf den Prozess der strukturellen Implementierung einer Personal- und Qualitätsabteilung würde dabei eines der Hauptaugenmerke gelegt werden. Es kann davon ausgegangen werden, dass in einem Unternehmen zumindest eine Personalabteilung vorhanden ist bzw., wenn nicht über eine Abteilung institutionalisiert, zumindest eine Person für Personalangelegenheiten verantwortlich ist. So müsste lediglich der Aufgabenbereich der Verantwortungsträger in diesem Bereich um die wissensmanagementtheoretischen Faktoren erweitert werden und bewusst die Bedeutung dieser Abteilung bzw. derjenigen Funktionsträger hervorgehoben werden. Einer Unternehmensführung stehen dafür Managementtechniken zur Verfügung.

Das Individuum als Ausgangspunkt sämtlicher wissensintensiver Prozesse stellt seine Intelligenz, seine Lernfähigkeit und sein Wissen dem Unternehmen zur Verfügung, wenn im Gegenzug das jeweilige Unternehmen diese individuellen Fähigkeiten und Fertigkeiten zu schätzen und zu verwenden weiß. Je besser Mitarbeiter in das Unternehmensgeschehen eingearbeitet sind und selbst Vorschläge zur unternehmerischen Weiterentwicklung geben können und dies auch tun, desto mehr kann sich ein Unternehmen von innen heraus verbessern. So gilt es von Seiten der Personalabteilung zu gewährleisten, dass Mitarbeiter bei Eintritt in ein Unternehmen bestens in ihren Arbeitsbereich und in das gesamte Unternehmen eingeschult werden und dass der Zweck der zu erfüllenden Aufgaben hinsichtlich der Erreichung von Unternehmenszielen klar verstanden wurde.

Dass Mitarbeiter reibungslos zusammenarbeiten können, wurde als eine Voraussetzung für ein effektives und erfolgreiches Wissensmanagement gewertet. Dies kann vor allem dann sichergestellt werden, wenn es institutionalisierte Handlungsabläufe in einem Unternehmen gibt, die idealerweise mit Hilfe einer guten 
technologischen Basisinfrastruktur für jeden einzelnen Mitarbeiter zugänglich sind und sich dadurch verwenden lassen. In dieser Arbeit wurde nicht weiter darauf eingegangen, wie die Handlungsabläufe eines Unternehmens institutionalisiert werden können. Weitere Untersuchungen könnten hier Aufschluss geben. Anhand eines Fallbeispiels könnte der Versuch gestartet werden, sämtliche Arbeitsvorgänge zur Erstellung der Produkte bzw. der Dienstleistungen eines Unternehmens zu erfassen und in Form sog. "best practices" in einer Datenbank zu speichern. Diese stellen noch nicht das organisationale Wissen dar, sondern „lediglich" die von den einzelnen Mitarbeitern eines bestimmten Aufgabenbereiches vorgeschlagenen Vorgehensweisen an bestimmte Sachverhalte und Problemstellungen.

Obwohl sich die genauen Aufzeichnungen der einzelnen Arbeitsschritte in einem Unternehmen als sehr zeit-, kosten- und personenaufwendig darstellen, basiert auf diesen der in dieser Arbeit entwickelte Wissensmanagement-Ansatz. Abhängig vom jeweiligen Unternehmen werden die Aufzeichnungen sicherlich unterschiedlich gemacht werden.

Da ein sensibler Bereich angesprochen wird, wenn Mitarbeiter ihr Wissen preisgeben sollen, sodass dieses als Informationen dem Unternehmen und somit anderen Mitarbeitern zur Verfügung steht, wird jedes Unternehmen für sich selbst entscheiden, wie sehr es die freie Gestaltung des Handlungsspielraums seiner Mitarbeiter durch die Externalisierung von individuellem Wissen einschränken möchte. Die Institutionalisierung dieser Handlungsabläufe erfolgt dann in einem eigenen Prozess, der wiederum über eine bestimmte Periode hinweg eigenständig untersucht werden müsste und könnte.

Basierend auf diesen Abläufen etablieren sich in einem Unternehmen formale Kommunikationsstrukturen. In Zusammenarbeit mit der strukturell integrierten Qualitätsabteilung unterliegen die Organisation wie die Mitarbeiter, Produkte oder Dienstleistungen eines Unternehmens einer ständigen Verbesserung und Weiterentwicklung. Das Zusammenspiel der innovativen Vorgänge Erne Enerungen 
und Umstrukturierungen innerhalb eines Unternehmens könnte ebenfalls ein interessantes und aufschlussreiches Forschungsgebiet darstellen.

Ein letzter Punkt, dem in der Arbeit selbst keine besondere Aufmerksamkeit geschenkt wurde, der jedoch empirisch sicherlich einen der relevantesten Forschungsschwerpunkte einnehmen würde, soll an dieser Stelle noch Erwähnung finden. Es ist die Frage des Nutzeneffekts von Wissensmanagement für das jeweilige Unternehmen. Zahlreiche Beiträge können hierzu in der einschlägigen Literatur bereits gefunden werden, die das Messen von Wissen und die Wertschöpfung eines Wissensmanagements zum Gegenstand haben (vgl. beispielsweise North, 2002; das Konzept der Wissensbilanz des Forschungszentrums Austrian Research Center Seibersdorf bei Bornemann/Leitner, 2001; Sveiby, 1997; Stewart, 1997; Roos et al., 1998; Kaplan/Norton, 1996; Skandia Group, 1997).

Auch der in dieser Arbeit entwickelte Wissensmanagement-Ansatz ist nur dann annähernd vollständig, wenn es einen Lösungsvorschlag für diese Fragestellung skizziert.

Im Zuge weiterer Forschungsarbeiten bzw. einer empirischen Überprüfung ließe sich der vorliegende Wissensmanagement-Ansatz weiter konkretisieren. Wissen selbst zu messen, ist auf Grund der hier zugrunde liegenden Arbeitsdefinition von Wissen, das handlungsrelevantes Wissen als Ausgangspunkt der Erläuterungen nimmt, nicht möglich.

Vielmehr könnten, wenn eine gesamtunternehmerische Perspektive in erwerbswirtschaftlichen Unternehmen eingenommen werden würde, die finanztechnischen Ergebnisse eines Unternehmens in Hinblick darauf beobachtet werden, auf welche Weise sie sich in der Periode vor der Einführung von Wissensmanagement bis nach der Einführung inklusive einer Dauer für die Normalisierung nach der Einführung entwickeln. Durch eine effiziente Organisation, die durch die genannten strukturellen Veränderungen erreicht werden s9ll und eine gute Zu- 
sammenarbeit der Mitarbeiter wird angestrebt, die Produkte und Dienstleistungen am Markt von innen heraus stetig zu optimieren und dadurch einen Wettbewerbsvorteil zu generieren.

Unter Zuhilfenahme der bereits bestehenden technologischen Möglichkeiten könnten zu den Handlungsabläufen und bezugnehmend auf den jeweiligen Zweck einer Arbeitsstelle Kennzahlen definiert werden. Somit würde die Möglichkeit bestehen, auf individueller Basis qualitative und quantitative Maße zu eruieren, wodurch der effiziente Beitrag eines Mitarbeiters gemessen werden könnte (vgl. Kaplan/Norton, 1996). Da strukturell und organisatorisch jeder Mitarbeiter direkt oder indirekt mit anderen Mitarbeitern eines Unternehmens verbunden ist, beeinflussen die einzelnen Leistungsbeiträge einen unternehmerischen Gesamtbeitrag. Dieser Gesamtbeitrag stellt jedoch nicht die Summe der Einzelteile dar, jedoch ist es einer der Bereiche, der sich von Seiten der Unternehmensführung als gestaltbar und steuerbar darstellt.

Informelle Netzwerke und Unternehmenskulturen können zwar von Seiten eines Unternehmens bewusst zu beeinflussen versucht werden, stoßen jedoch an unüberwindbare Grenzen der Messung. Um die organisatorische Gestaltung daher einfach zu halten, wäre es eine Möglichkeit, die Leistung von Mitarbeitern so zu messen, als der individuelle Beitrag zur Erfüllung eines Unternehmenszwecks näher analysiert wird.

Die Qualität der Messung kann jedoch lediglich so gut sein wie die Qualität der Herstellung von kausalen Zusammenhängen zwischen den einzelnen Beiträgen und dem gesamtunternehmerischen Ergebnis. Ohne diesen Bereich näher erläutern zu wollen, stößt man auf zahlreiche Problemfelder, da beispielsweise bereits eine Kausalität zwischen individuellem Input und organisationalem Output lediglich schwer, wenn überhaupt herzustellen ist. Es wäre jedoch für weitere Forschungsarbeiten sicherlich interessant, ausschließlich diesen einen Aspekt der Messung der Effizienz eines Wissensmanagement-Ansatzes zu erläutern. 


\section{Zusammenfassung und Ausblick}

Der beste Weg,

die Zukunft vorauszusagen, ist, sie zu gestalten.

Unbekannt

Mit der vorliegenden Arbeit wurde das Ziel verfolgt, einen WissensmanagementAnsatz zu elaborieren, der die Organisation von Unternehmen in den Mittelpunkt der Betrachtung lenkt, um ein institutionelles Rahmengerüst zu schaffen, mithilfe dessen Individuen als Träger und Quelle von Wissen ihre eigenen Präferenzstrukturen auf die Nutzenfunktion eines Unternehmens ausrichten und ihr Wissen im Sinne der Unternehmensziele einsetzen.

Die Literatur in diesem Bereich ist noch stark durch funktionalistische Ideen geprägt. Es gilt vor allem Wege zu finden, die es Unternehmen ermöglichen, effektiver und effizienter als ihre Mitbewerber zu agieren, wodurch sich diese einen komparativen Vorteil schaffen können. Eine funktionalistische Verortung bringt wissenschaftstheoretische Implikationen mit sich, die in Kapitel 3 mit einer Gegenüberstellung zu Annahmen einer interpretativen Logik erläutert werden. Die vorliegende Arbeit beleuchtet die Probleme und Defizite von Wissensmanagement aus einem funktionalistischen Paradigma und stellt sie Ideen eines interpretativen Paradigmas gegenüber, indem die Teilbereiche "Wissen", "Managen" sowie "Wissen managen" aufgearbeitet werden (Kapitel 3).

Der Forschungsbereich Wissensmanagement stellt sich als sehr inhomogen dar, daher wurde Kapitel 4 dem State-of-the-Art gewidmet. Die sieben in der betriebswirtschaftlichen Literatur gängigsten Wissensmanagement-Ansätze wurden aufgeführt und dargestellt. Die innen inhärenten Unzulänglichkeiten leiten die Entwicklung eines eigenständig zu entwickelnden WissensmanagementAnsatzes ein. 
Das Fundament des elaborierten Wissensmanagement-Ansatzes bildet die Theorie der Neuen Institutionenökonomie. Sie verspricht aufgrund ihrer Grundannahmen des methodologischen Individualismus sowie ihrer Verhaltensannahme der beschränkten Rationalität als eine Basis eines Wissensmanagement-Ansatzes, der den Fokus auf die Organisation von Unternehmen sowie die Rolle des Individuums legt, geeignet zu sein. Die drei Hauptströmungen innerhalb dieses Theorienkomplexes bieten ausreichend Aufschluss darüber, welche Auswirkungen Institutionen auf das menschliche Verhalten haben und wie ein effizientes Design von Institutionen gestaltet werden sollte (Kapitel 5).

Die Quelle von Wissen wird in den Köpfen von Individuen ausgemacht. Andererseits ist Wissen auch in den personenunabhängigen anonymisierten Regelsystemen, Standardverfahrensweisen, Normen, institutionalisierte Handlungsabläufe etc. eingebettet. Auf welche Weise Institutionen gestaltet werden sollten, um das im Unternehmen konstatierte Organisationsproblem, das das Koordinations- und Motivationsproblem von Mitarbeitern umfasst, zu überwinden, wird in Kapitel 6 der vorliegenden Arbeit näher erläutert. Ein erfolgreiches Wissensmanagement zeichnet abhängig vom Können und Wollen der Mitarbeiter eines Unternehmens. Mit der integrativen Verknüpfung der Theorie der Neuen Institutionenökonomie sowie des Forschungsbereichs Wissensmanagement kann diese Problematik adressiert werden.

Wissensmanagement sollte eine Querschnittsfunktion erfüllen und nicht lediglich an einer Stelle eines Unternehmens versickern. Der in Kapitel 6 entwickelte Entwurf eines Wissensmanagement-Ansatzes fokussiert das in einem Unternehmen bereits vorhandene Wissensmanagement, das unbewusst und unstrukturiert in einem beliebigen Unternehmen vorzufinden ist. So soll der vorliegende Ansatz eine Möglichkeit bieten, das bereits vorhandene Wissensmanagement eines Unternehmens zu strukturieren, indem institutionelle Erweiterungen und Schwerpunktsetzungen in Form einer Personal- und Qualitätsabteilung, die den Fokus auf das Wissen der Mitarbeiter legen, durchgeführt werden. 
In Zukunft scheinen diejenigen Unternehmen erfolgreich zu sein, die es auf intelligente und effektive Weise verstehen, ihr jeweils vorhandenes organisationales Wissen bewusst zu machen, zu nutzen und wirtschaftlich zu verwerten. Zur zukunftsorientierten Navigations- und Überlebensfähigkeit von Unternehmen gehört das Überwinden des Organisationsproblems, das das Motivations- sowie Koordinationsproblem umfasst.

Obwohl von der Theorie der Neuen Institutionenökonomie brauchbare Konzepte und Ideen als Anregungen für die Entwicklung eines WissensmanagementAnsatzes übernommen werden können, stößt man jedoch auch mit einem solchen theoretischen Fundament auf gewisse Grenzen. So kann nicht klar definiert werden, auf welche Weise eine Personal- bzw. Qualitätsabteilung individuelle Nutzenfunktionen in Verträgen berücksichtigen sollte, um ein für ein Unternehmen satisfizierendes Können und Wollen von Individuen zu erreichen. Da die Ausrichtung der individuellen Präferenzstrukturen auf die Nutzenfunktion eines Unternehmens jedoch als ein Schlüsselpunkt eines WissensmanagementAnsatzes fungiert, stellt obig erwähnte Diskrepanz ein nicht zu unterschätzendes fundamentales theoretisches Problem dar.

Eine vertiefende Auseinandersetzung und Verfeinerung des entwickelten Wissensmanagement-Ansatzes könnte Aufschluss darüber geben, auf welche Weise individuelle Nutzenfunktionen vertraglich geregelt werden sollen und welche Anreiz- und Sanktionsmechanismen unterstützend wirksam werden können. 


\section{Literaturverzeichnis}

Adorno, T.W., H. Albert, R. Dahrendorf, J. Habermas, H. Pilot, und K.R. Popper: Der Positivismusstreit in der deutschen Soziologie, 5. Aufl., Darmstadt, 1976.

Albrecht, F.: Strategisches Management der Unternehmensressource Wissen: Inhaltliche Ansatzpunkte und Überlegungen zu einem konzeptionellen Gestaltungsrahmen, Dissertationsschrift, Frankfurt/Main, 1993.

Alchian, A.A. and H. Demsetz: The Property Right Paradigm, in: Journal of Economic History, 1973, 33, 16-27.

Alvesson, M. and D. Kärreman: Odd Couple: Making sense of the curious concept of knowledge management, in: Journal of Management Studies 38:7, November 2001, 9951018.

Andreu, R. and C. Ciborra: Core Capabilities and Information Technology: An Organizational Learning Approach, in: Edmondson, A., Moingeon, B. (Eds.): Organizational Learning and Competitive Advantage, Surrey, 1996, 121-138.

Appelbaum, S.H. and J. Gallagher: The Competitive Advantage of Organizational Learning, in: Journal of Workplace Learning: Employee Counselling Today, 2000, 12, 2, 40-56.

Argyris, C. and D. Schön: Organizational Learning: A Theory of Action Perspective. Reading, Mass.: Addison-Wesley, 1978.

Arrow, K.J.: The Limits of Organization, New York, 1970.

Barley, S.R. and P.S. Tolbert: Institutionalization and Structuration: Studying the links between action and institution, in: Organization Studies, 1997, 93-117.

Barney, J.B.: The debate between traditional management theory and organizational economics: Substantive differences or intergroup conflict?, in: Academy of Management Review, 1990, 15, 382-393.

Barney, J.B: Firm resources and sustained competitive advantage, in: Journal of Management, 17, 1991, 99-120.

Becker, M.C.: Managing Dispersed Knowledge: Organizational problems, managerial strategies, and their effectiveness, in: Journal of Management Studies 38:7, November 2001, 1037-1051.

Bending, R.: Aspekte der konzeptionellen Modellierung eines wissensbasierten Planungssystems zur strategischen Unternehmensplanung, Diss.schrift, Duisberg, 1988.

Berger, P. and Th. Luckmann: Die gesellschaftliche Konstruktion der Wirklichkeit. 16th edition, Fischer: Frankfurt am Main, 1999. 
Blackler, F.: Knowledge, Knowledge Work and Organizations: An Overview and Interpretation, in: Organization Studies, 1995, 1021-1046.

Blackler, F., N. Crump and S. McDonald: Knowledge, Organizations and Competition, in: von Krogh, G., Roos, J., Kleine, D. (Eds.): Knowing in Firms: Understanding, Managing and Measuring Knowledge. Gateshead, 1998, 67-86.

Blosch, M.: Pragmatism and Organizational Knowledge Management, in: Knowledge and Process Management 2001, Vol. 8, No. 1, 39-47.

Boland, R.J.Jr. and R.V. Tenkasi: Perspective making and perspective taking in communities of knowing, in: Organization Science, 1995, 6/4, 350-372.

Bowditch, J.L. and A.F. Buono: A primer on organizational behavior, $2^{\text {nd }}$ ed., Canada, 1990.

Brown, J.S. and P. Duguid: Organizational Learning and Communities of Practice: Toward a unified view of Working, Learning, and Innovation, in: Organization Science, Vol. 2, No. 1, February 1991, 40-56.

Bruner, J.: Acts of meaning, Cambridge, 1990.

Burrell, G. and G. Morgan: Sociological Paradigms and organisational analysis. Sage: London, 1979.

Chandler, A.: Strategy and Structure: Chapters in the History of the Industrial Enterprise, Cambridge (Mass.), 1962.

Coase, R.: The nature of the firm (Originalbeitrag in: Economica, 1937, No.4, 386-405), in: Williamson, O.E. and S.G. Winter: The nature of the Firm, Origins, Evolution, and Development, Oxford, 1993, 18-33.

Coff, R.: Human Assets and Management Dilemmas: Coping with Hazards on the Road to Resource-Based Theory, in: Academy of Management Review 1997, Vol. 22, No. 2 , 374-402.

Cohen, D. and L. Prusak: In good company: how social capital makes organizations, Bosten, Mass., 2001.

Collis, D.: Organizational Capability as a Source of Profit, in: Edmondson, A., Moingeon, B. (Eds.): Organizational Learning and Competitive Advantage, Surrey, 1996, 139-163.

Cook, S. and J.S. Brown: Bridging Epistemologies: The Generative Dance Between Organizational Knowledge and Organizational Knowing, in: Organization Science, Vol.10, No. 4, July-August 1999, 381-400.

Cyert, R.M. and J.G. March: A behavioral theory of the firm, 2. Aufl., New York, 1963.

Czarniawska, B.: Narrating the Organization: Dramas of Institutional Identity, Chicago, 1997. 
Czarniawska, B.: A Narrative Approach to Organization Studies, Qualitative Research Methods Series 43, United States of America, 1998.

Davenport, T. und L. Prusak: Working knowledge: how organizations manage what they know, Harvard Business School Press, 1998.

Davenport, T. und L. Prusak: Wenn Ihr Unternehmen wüßte, was es alles weiß...Das Praxishandbuch zum Wissensmanagement, Landsberg/Lech, 1998.

Deetz, S.: Democracy in an Age of Corporate Colonization: Developments in Communication and the Politics of Everyday Life, New York, 1992.

Demsetz, H.: Toward a theory of property rights, in: American Economic Review, 1967, 57, 347-359.

Denning St.: The Springboard: How Storytelling Ignites Action in Knowledge-Era Organizations, Boston, 2001.

DiMaggio, P.J. and W.W. Powell: Introduction, in: Powell, W. W. and P. J. DiMaggio (Eds.): The New Institutionalism in Organizational Analysis. London, 1991, 1-38.

Dodgson, M.: Organisational Learning: a review of some literatures, in: Organisation Studies, 14/3, 1993, 375 - 394.

Donaldson, L.: The ethereal hand: Organizational economics and management theory, in: Academy of Management Review, 1990, 15, 369-381.

Drobak, J.N. and J.V.C. Nye: The frontiers of the new institutional economics, San Diege, 1997.

Drucker, P.: The new society of organizations, in: Harvard Business Review, Vol.70, No. 5. September-October 1992.

Drucker, P.: Post-Capitalist Society, Oxford, 1993.

Duncan, R. and A. Weiss: Organizational Learning: Implications for Organizational Design, in: Research in Organizational Behavior, No. 1, 1979, 75-123.

Earl, M.J.: Information Management: The Organizational Dimension, New York, 1996.

Easterby-Smith, M.: Disciplines of Organizational Learning: Contributions and Critiques, in: Human Relations, 50/9, 1997, 1085-1113.

Easterby-Smith, M. and L. Araujo: Organizational Learning: Current Debates and Opportunities, in: Easterby-Smith, M., J. Burgoyne and L. Aurajo: Organizational Learning and the Learning Organization. Developments in theory and practice, Sage: Thousand Oaks, $1999,1-21$.

Ebers, M. und W. Gotsch: Institutionenokonomische Theorien der Organisation, in: Kieser, A. (Eds.): Organisationstheorien. 3. überarb. und erw. Aufl., Stuttgart, 1999, 199-251. 
Eckardstein, von D.: Das EFQM-Modell des Qualitătsmanagements, in: Eckardstein, v.D., H. Kasper und W. Mayrhofer (Hrsg.): Management, Theorien-Führung-Veränderung, Stuttgart, 1999, 421-430.

Edeling, Th.: Einführung: Der Neue Institutionalismus in Okonomie und Soziologie, in: Edeling, Th., W. Jann und D. Wagner (Eds.): Institutionenokonomie und Neuer Institutionalismus. Überlegungen zur Organisationstheorie, Opladen, 1999, 7-15.

Edmondson, A. and B. Moingeon: Introduction: Organizational Learning as a Source of Competitive Advantage, in: Edmondson, A., Moingeon, B. (Eds.): Organizational Learning and Competitive Advantage, Surrey, 1996, 7-15.

Edmondson, A. and B. Moingeon: When to Learn How and When to Learn Why: Appropriate Organizational Learning Processes as a Source of Competitive Advantage, in: Edmondson, A., B. Moingeon (EDS.): Organizational Learning and Competitive Advantage, Surrey, 1996, 17-37.

Elkjaer, B.: In Search of a Social Learning Theory, in: Easterby-Smith, M., J. Burgoyne and $L$. Aurajo: Organizational Learning and the Learning Organization. Developments in theory and practice, Sage: Thousand Oaks, 1999, 75 - 91.

Engeström, Y.: Comment on Blackler et al. Activity Theory and the Social Construction of Knowledge: A Story of Four Empires, in: Organization, 7/2, 301-310.

Eppler, J.M. und O. Sukowski (Eds.): Fallstudien zum Wissensmanagement: Losungen aus der Praxis, St. Gallen, 2001.

Eschenbach, R. (Ed.): Controlling, 2.überarb. und erw. Aufl., Stuttgart, 1996.

Esser, H..: Wie oft noch? Wie lange noch?, in: Ortmann, G.; J. Sydow; K. Türk (Eds.): Theorien der Organisation: Die Rückkehr der Gesellschaft. Opladen, 1997, 259-262.

Fiol, M.C.: Consensus, Diversity, and Learning in Organization, in: Organization Science, Vol. 5, No. 3, August 1994, 403-420.

Föhr, S.: Okonomische Analyse der internen Organisation, Wiesbaden, 1991.

Foss, N.J.: Resources, Firms, and Strategies, A Reader in the Resource-Based Perspective, Oxford University Press, 1997.

Foss, N.J. (Ed.): The Theory of the Firm: Critical perspectives on business and management, Volume I, Part I - Part II, New York, 2000.

Foss, N.J. (Ed.): The Theory of the Firm: Critical perspectives on business and management, Volume II, Part III - Part IV, New York, 2000.

Foss, N.J. (Ed.): The Theory of the Firm: Critical perspectives on business and management, Volume III, Part V - Part VI, New York, 2000.

Foss, N.J. (Ed.): The Theory of the Firm: Critical perspectives on business and management, Volume IV, Part VII - Part IX, New York, 2000. 
Frey, B.S und M. Osterloh: Pay for Performance - Immer empfehlenswert, 2000.

Friedmann, M: Essays in positive economics, Chicago, 1953.

Friedman, V.J.: The Individual as Agent of Organizational Learning, in: Dierkes M., A.B. Antal, J. Child and I. Nonaka (Eds.): Handbook of Organizational Learning and Knowledge, Oxford, 2001, $398-414$.

Furubotn, E.G. and R. Richter: Institutions and Economic Theory: the contribution of the new institutional economics, Michigan, 2000.

Gabriel, Y.: Storytelling in Organisations, Oxford, 2001.

Göhler, G. und R. Kăhn: Institutionenökonomie, Neo-Institutionalismus und die Theorie politischer Institutionen, in: Edeling, T., W. Jann und D. Wagner (Eds.): Institutionenokonomie und Neuer Institutionalismus: Überlegungen zur Organisationstheorie, Opladen, $1999,17-42$.

Gälweiler, A.: Determinanten des Zeithorizonts in der strategischen Planung, in: Hahn, D. und B. Taylor (Eds.): Strategische Unternehmensplanung - strategische Unternehmensführung, 5. Aufl., Heidelberg 1990, 203-220.

Gherardi, S.: Practice-based Theorizing on Learning and Knowing in Organizations, in: Organization, $7 / 2,211-223$.

Gherardi, S. and D. Nicolini:The Sociological Foundations of Organizational Learning, in: Dierkes M., A.B. Antal, J. Child and I. Nonaka (Eds.): Handbook of Organizational Learning and Knowledge, Oxford, 2001, 35-60.

Grant, R.M.: Toward a Knowledge-based Theory of the Firm, in: Strategic Management Journal, 1996, 17, (Winter special issue), 109-122.

Grant, R.M.: Knowledge and Organization, in: Nonaka, I., D.J. Teece (Eds.): Managing Industrial Knowledge, Creation, Transfer and Utilization, London, 2001, 145-169.

Gherardi, S.: Organizational Learning, in: M. Warner (Ed.): International Encyclopedia of Business and Management, Routledge: London, 1996, 3934-3942.

Gherardi, S.: Practice-based Theorizing on Learning and Knowing in Organizations, in: Organization, 2000, 211-223.

Gherardi, S., D. Nicolini and F. Odella: Toward a social understanding of how people learn in organizations, in: Management Learning, 1998, 29/3, 273-297.

Goia, D. and E. Pitre: Multiparadigm perspectives on theory building, in: Academy of Management Review, 1990, 15 (4), 584-602.

Goshal, S. and P. Morgan: Bad for practice: A critique of the transaction cost theory, in: Academy of Management Review, 1996, 21, 13-47. 
Greenwood, R. and C.R. Hinings: Understanding radical organizational change: Bringing together the old and the new institutionalism, in: The Academy of Management Review, $1996,1022-1054$.

Griseri, P.: Management knowledge: a critical view, Wales, 2002.

Güldenberg, S.: Wissensmanagement und Wissenscontrolling in lernenden Organisationen: ein systemtheoretischer Ansatz, Dissertationsschrift, 2., durchges. Aufl., Wiesbaden, 1998.

Güldenberg, S.: Wissensmanagement, in: ControllerNews, 1997, Heft 4, 10-11.

Hamel, G. and C.K Prahalad: Competing for the Future, Boston, 1994.

Hansen, M.T., N. Nohria and Th. Tierney: What's your strategy for managing knowledge?, in: Harvard Business Review, March-April 99, 106-116.

Hasse, R. und G. Krücken: Was leistet der organisationssoziologische NeoInstitutionalismus? Eine theoretische Auseinandersetzung mit besonderer Berucksichtigung des wissenschaftlichen Wandels, in: Soziale Systeme 2, 1996, 91-112.

Huemer, L., G. von Krogh and J. Roos: Knowledge and the concept of trust, in: von Krogh, G., Roos, J., Kleine, D. (Eds.): Knowing in Firms: Understanding, Managing and Measuring Knowledge. Gateshead, 1998, 123-145.

Hunt, J.G.: Leadership a new Synthesis, Sage, 1991.

Jablin, F.M. and P.M. Sias: Communication Competence, in: Jablin, F.M., Putnam, L.L. (Eds.): The New Handbook of Organizational Communication, Advances in Theory, Research, and Methods, California, 2001, 819-864.

Jensen, M.C. and W.H. Meckling: Specific and General Knowledge, and Organizational Structure, in: L. Werin and H. Wijkander (Eds.): Contract Economics, Oxford, 1992, 251274.

Kakabadse, N.K. and A. Kouzmin and A. Kakabadse: From Tacit Knowledge to Knowledge Management: Leveraging Invisible Assets, in: Knowledge and Process Management, 2001, Vol. 8, No. 3, 137-154.

Kant, I.: Kritik der reinen Vernunft, Leipzig, 1971.

Kaplan, R.S. and D.P. Norton: The Balanced Scorecard. Translating Strategy into Action, Boston/Mass., 1996.

Kasper, H. und P. Heimerl-Wagner: Struktur und Kultur in Organisationen, in: Kasper, $\mathrm{H}$. und W. Mayerhofer (Eds.): Personalmanagement, Führung, Organisation, 2. Aufl., Wien, 1996, S. 9-108. 
Kasper, H. und W. Mayrhofer und M. Meyer: Management aus systemtheoretischer Perspektive - eine Standortbestimmung, in: Eckardstein, D., H. Kasper und W. Mayerhofer (Eds.): Management: Theorien - Führung - Veränderung, Wien, 1999, S. 161-209.

Kappelhoff, P.: Rational Choice, Macht und die korporative Organisation der Gesellschaft, in: Ortmann, G.; J. Sydow; K. Türk (Eds.): Theorien der Organisation: Die Rückkehr der Gesellschaft. Opladen, 1997, 218-258.

Kieser, A.: Organisationstheorien, 3. überarb. und erw. Aufl., Stuttgart, 1999.

Kieser, A.: Implementierungsmanagement im Zeichen von Moden und Mythen des Organisierens, in: Nippa, M./Scharfenber, H. (Ed.): Implementierungsmanagement: Über die Kunst, Reengineeringkonzepte erfolgreich umzusetzen, Wiesbaden 1997, S. 79-102.

Kieser, A. und H. Kubicek: Organisation, 3. überarb. und erw. Aufl., Stuttgart u.a., 1999.

Kirsch, W.: Wegweiser zur Konstruktion einer evolutionären Theorie der strategischen Unternehmensführung, Kapitel eines Theorieprojektes, München, 1996.

Klimecki, R.G.: Wissensmanagement - Wege zur "intelligenten Organisation", Nr. 30, Diskussionsbeitrag Dezember 1999.

Knorr-Cetina, K.: Epistemic cultures. How the Sciences Make Knowledge, Cambridge, 1999.

Knyphausen-Aufsess, D. zu: Theorie der strategischen Unternehmensführung: State of the art und neue Perspektiven, Wiesbaden, 1995.

Kogut, B. and U. Zander: Knowledge of the Firm, Combinative Capabilities, and the Replication of Technology, in: Foss, N.J. (Ed.): Resources, Firms, and Strategies. A Reader in the Resource-Based Perspective, Oxford, 1999, 306-326.

Kotorov, R.P.: Virtual Organization: Conceptual Analysis of the Limits of its Decentralization, in: Knowledge and Process Management 2001, Vol. 8, No. 1, 55-62.

Kramer, R.M. and T.R. Tyler (Eds.): Trust in Organizations: Frontiers of theory and research, California, 1996.

Krogh, G. von and J. Roos: Five claims on knowing, in: European Management Journal 1996, Vol. 14; No. 4, 417-436.

Krogh, G. von, J. Roos and D. Kleine (Eds.): Knowing in Firms, London, 1998.

Krogh, G. von, K. Ichijo and I. Nonaka (Eds.): Enabling Knowledge Creation. How to Unlock the Mystery of Tacit Knowledge and Release the Power of Innovation, Oxford, Univ. Press, 2000.

Kuhn, T.S.: Die Struktur wissenschaftlicher Revolutionen, 2. rev. Aufl., Frankfurt, 1976.

Lam, A.: Tacit Knowledge, Organizational Learning and Societal Institutions: An Integrated Framework, in: Organization Studies 2000, 21/3, 487-513. 
Lang, R., I. Winkler und E. Weik: Organisationales Lernen, in: Weik, E. und R. Lang (Hrsg.): Moderne Organisationstheorien, Wiesbaden, 2001, 255-284.

Leonard-Barton, D.: Wellsprings of Knowledge, Boston, 1995.

Lueger, M.: Grundlagen qualitativer Feldforschung. Methodologie - Organisierung Materialanalyse. Wien: WUV 2000.

Maglhães, R.: Organizational Knowledge and Learning, in: von Krogh, G., Roos, J., Kleine, D. (Eds.): Knowing in Firms: Understanding, Managing and Measuring Knowledge. Gateshead, 1998, 87-122.

Malhotra, Y.: Why Knowledge Management Systems Fail? Enablers and Constraints of Knowledge Management in Human Enterprises, forthcoming in: Handbook on Knowledge Management (Holsapple, C.W. (Ed.)), Heidelberg, 2002.

Maletzke, G.: Kommunikationswissenschaften im Überblick: Grundlagen, Probleme, Perspektiven, Wiesbaden, 1998.

March, J.G. and J.P. Olsen: Ambiguity and Choice in Organizations, Bergen, 1976.

March, J.G.: Exploration and Exploitation in Organizational Learning, in: Cohen, M.D., Sproull, L.S. (Eds.): Organizational Learning, 1996, 101-123.

Mayerhofer, W.: Motivation und Arbeitsverhalten, in: Kasper, H. und W. Mayerhofer (Eds.): Personalmanagement, Führung, Organisation, 3. Aufl., Wien, 2002, S. 255-288.

Ménard, C.: Institutions, Contracts and Organizations: perspectives form new institutional economics, Cheltenham, 2000.

Meuthen, D.: Neue Institutionenökonomik und strategische Unternehmensführung, Aachen, Univ., Diss.schrift, 1997.

Meyer, R.: Die Konstruktion der Umwelt von Organisationen. Service Fachverlag: Wien, 1996.

Meyer, J.W. and B. Rowan: Institutionalized Organizations: Formal Structure as Myth and Ceremony, in: J.W. Meyer and W.R. Scott (Eds.): Organizational Environments. Ritual and Rationality. Beverly Hills, 1983, $21-44$.

Milgrom, P. and J. Roberts: Economics, Organization, and Management, Englewood Cliffs, 1992.

Morgan, G.: Images of Organization, Beverly Hills, 1986.

Mumby, D.K.: Power and Politics, in: Jablin, F.M., Putnam, L.L. (Eds.): The New Handbook of Organizational Communication, Advances in Theory, Research, and Methods, California, 2001, 585-623.

Nanda, A.: Resources, Capabilities and Competencies, in: Edmondson, A., Moingeon, B. (Eds.): Organizational Learning and Competitive Advantage, Surrey, 1996, 92-120. 
Nelson, R. and S. Winter: An Evolutionary Theory of Economic Change, London, 1982.

Neumann, R.: Die Organisation als Ordnung des Wissens: Wissensmanagement im Spannungsfeld von Anspruch und Realisierbarkeit, Wiesbaden, 2000; (zugl.: Klagenfurt, Univ., Habil.-Schr., 2000).

Nonaka, I.: A dynamic theory of organizational knowledge creation, in: Organization Science, 1994, 5 (1), 14-37.

Nonaka, I. and H. Takeuchi: The Knowledge-Creating Company, Oxford, 1995.

North, D.C: Structure and change, in: Economic History, New York, 1981.

North, D.C: Institutions, institutional change and economic performance, Cambridge, 1990.

North, D.C: Economic performance through time, in: Brinton, M.C. and V. Nee (Eds.): The new institutionalism of sociology, New York, 1998, 247-257.

North, K.: Wissensorientierte Unternehmensführung, Wertschöpfung durch Wissen, 3.Aufl., Wiesbaden, 2002.

Ostrom, E.: Governing the Commons. The Evolution of Institutions for collective Action, Cambridge, 1990.

Pappenheim, R.: Neue Institutionenokonomik und politische Institutionen: zur Anwendung der okonomischen Theorie auf politische Institutionen und Organisationen, Frankfurt am Main, 2001.

Pawlowsky, P.: Betriebliche Qualifikationsstrategien und organisationales Lernen, in: Staehle, W.H. und P. Conrad (Hrsg.): Managementforschung 2, Berlin, 1992, 177-237.

Pawlowsky, P.: Wissensmanagement in der lernenden Organisation, Habilitationsschrift, Paderborn, 1994.

Pawlowsky, P. und J. Bauumer: Betriebliche Weiterbildung, Management von Qualifikation und Wissen, München, 1996.

Pautzke, G.: Die Evolution der organisatorischen Wissensbasis. Bausteine zu einer Theorie des organisatorischen Lernens, München, 1989.

Penrose, E.T.: The Theory of the Growth of the Firm, New York, 1959.

Pfeffer, J. and G.R Salancik: The external control of organizations: A resource dependence perspective, New York, 1978.

Peteraf, M.: The Cornerstones of Competitive Advantage: A Resource-based View, in: Strategic Management Journal 14, 1993, 179-192.

Pfeffer, X. and X. Sutton: The knowing-doing Gap, Boston: Harvard Business School Press. 
Picot, A. and E. Schlicht (Eds.): Firms, Markets, and Contracts: Contributions to Neoinstitutional Economics, Heidelberg, 1996.

Picot, A., H. Dietl und E. Franck: Organisation, eine okonomische Perspektive, 2., überarb. und erw. Aufl., Stuttgart, 1999.

Picot, A., R. Reichwald und R.T. Wigand: Die Grenzenlose Unternehmung - Information, Organisation und Management, 3., überarb. Aufl., Wiesbaden, 1998.

Pinchot, G. and E. Pinchot: The Rise and Fall of Bureauchracy, in: Myers, P. (Ed.): Knowledge Management an Organizational Design, Boston, 1996, 39-53.

Pirker, R.: Die Unternehmung als soziale Institution: Eine Kritik der Transaktionskostenerklärung der Firma, in: Ortmann, G.; J. Sydow; K. Turk (Eds.): Theorien der Organisation: Die Rückkehr der Gesellschaft. Opladen, 1997, 67-80.

Polanyi, M.: Personal knowledge: towards a post-critical philosophy, New York, Harper Torchbooks, 1962.

Porter, M.: Competitive Advantage of Nations, New York, 1985.

Powell, W.W. and Paul J. DiMaggio (Eds.): The New Institutionalism in Organizational Analysis, London, 1991.

Prahalad, C.K. and G. Hamel: The core competence of the corporation, in: Harvard Business Review, May-June 1990, 71-91.

Prange, C.: Organizational Learning - Desperately Seeking Theory?, in: Easterby-Smith, M., J. Burgoyne and L. Aurajo: Organizational Learning and the Learning Organization. Developments in theory and practice, Sage: Thousand Oaks, 1999, 23-43.

Probst, G., Büchel B. and S. Raub: Knowledge as a Strategic Resource, in: von Krogh, G., Roos, J., Kleine, D. (Eds.): Knowing in Firms: Understanding, Managing and Measuring Knowledge. Gateshead, 1998, 240-252.

Probst, G. and Bettina S.T. Büchel: Organizational Learning: The competitive advantage for the future. Prentice Hall: London, 1997.

Probst, G., S. Raub und K. Romhardt: Wissen managen: Wie Unternehmen ihre wertvollste Ressource optimal nutzen, Wiesbaden, 1997.

Prusak, L.: Knowledge in Organizations, Oxford, 1998.

Prusak, L. and D. Cohen: How to invest in Social Capital, in: Harvard Business Review, June, 2001, 86-93.

Quinn, J.: Intelligent enterprise. A knowledge and service based paradigm for industry, New York, 1992.

Rau-Bredow, H.: Zur theoretischen Fundierung der Institutionenökonomie, München, Univ., Diss., 1992. 
Rehäuser, J. und H. Krcmar: Wissensmanagement im Unternehmen, in: G. Schreyogg und P. Conrad (Eds.): Managementforschung 6: Wissensmanagement, Berlin, 1996, 140.

Rheilen, M. und K. Sikora: Phänomenologischer versus technologischer Ansatz für das Wissensmanagement in Unternehmen - Eine kritisch-konstruktive Auseinandersetzung mit J.-C. Spenders Konzept der Knowledge-based Theory of the Firm, in: Schreyogg, G. (Hrsg.): Wissen in Unternehmen: Konzepte, Maßnahmen, Methoden, 1. Aufl., Berlin, 2001, 119-162.

Richter, R.: Institutionen okonomisch analysiert: Zurr jüngeren Entwicklung auf dem Gebiet der Wirtschaftstheorie, Dillingen, 1992.

Richter, R. und E.G. Furubotn: Neue Institutionenokonomie: eine Einführung und kritische Würdigung, Tübingen, 1996.

Roberts, P.W. and R. Greenwood: Integrating transaction cost and institutional theories: Towards a constrained-efficiency framework for understanding organizational adoption, in: Academy of Management Review, 1997, 22, 346-373.

Roehl, H.: Organisationen des Wissens: Anleitung zur Gestaltung, Stuttgart, 2002.

Roehl, H.: Instrumente der Wissensorganisation. Perspektive für eine differenzierende Interventionspraxis, Diss.schrift, Wiesbaden, 2000.

Roos, J. et al: Intellectual Capital, New York, 1998.

Rosenstiel, L.V.: Grundlagen der Organisationspsychologie, Stuttgart, 1987.

Rickson, R.E.: Knowledge Management in Industrial Society and Environment Quality, in: Human Organization, 35/76, 239-251.

Schanz, G.: Methodologie für Betriebswirte, 2., überarb. u. erw. Aufl., Stuttgart, 1988.

Scheer, A.W.: ARIS, Vom Geschaftsprozess zum Anwendungssystem, Berlin, 1998.

Scherer, A.G.: Pluralism and Incommensurability, in: Strategic management and Organization Theory: A Problem in Search of a Solution, Organization, 1998, 5 (2), 147-168.

Scherer, A.G.: Kritik der Organisation oder Organisation der Kritik? - Wissenschaftstheoretische Bemerkungen zum kritischen Umgang mit Organisationstheorien, in: Kieser, A. (Hrsg.): Organisationstheorien, 3. überarb. und erw. Aufl., Stuttgart, 1999, 1-38.

Scheuble, S.: Wissen und Wissenssurrogate: eine Theorie der Unternehmung, München, Univ., Diss.schrift, 1998.

Schick, H.: Theorieprobleme des Wissensmanagements, in: Zeitschrift für Personalforschung, 16. Jg., Heft 3, 2002, 433- 458.

Schoen, S.: Gestaltung und Unterstützung von Communities of Practice, München, 2000; (zugleich Diss.schrift, München, 2000). 
Schreyögg, G. und P. Eberl: Organisationales Lernen: Viele Fragen und noch zu wenig neue Antworten, in: Die Betriebswirtschaft, 58/4, 1998, 516-536.

Schreyögg, G. und D. Geiger: Wenn alles Wissen ist, ist Wissen am Ende nichts?!, in: Die Betriebswirtschaft, 63, 2003, 7-22.

Schultze, U.: Investigating the Contradictions in Knowledge Management, in: T.J. Larsen, L. Levine and J.I. DeGross (Eds.): Information Systems: Current Issues and Future Changes, IFIP, Laxenberg, 1999, S. 155-174.

Schüppel J., Müller-Stewens G. and Gomez P.: The Knowledge Spiral, in: von Krogh, G., J. Roos, and D. Kleine (Eds.): Knowing in Firms: Understanding, Managing and Measuring Knowledge. Gateshead, 1998, 26-66.

Scott, W. R.: Institutions and Organizations. Toward a Theoretical Synthesis, in: W.R. Scott und J.W. Meyer (Eds.): Institutional Environments and Organizations; Structural Complexity and Individualism, Thousand Oaks, 1994, 55 - 80.

Scott, W. R. (1995a): Introduction: Institutional Theory and Organizations, in:, R.W. Scott und S. Christensen (Eds.): The Institutional Construction of Organizations. International and Longitudial Studies. Thousand Oaks, 1995.

Scott, W. R. (1995b): Institutions and Organizations. Thousand Oaks, 1995.

Seng, P.: Information und Versicherung: Produktionstheoretische Grundlagen, Wiesbaden, 1989.

Senge, P.: The Fifth discipline: the art and practice of the learning organization, London, 1993.

Senge, P.: The Dance of Change. Die 10 Herausforderungen tief greifender Veränderungen in Organisationen, Wien/Hamburg, 2000.

Settler, M.: Institutions, Property Rights and External Effects: New Institutional Economics and the Economics of John R. Commons, Zürich, Univ.Diss., 1999.

Shrivastava, P.: A typology of Organizational Learning Systems, in: Academy of Management Studies, 20/1, 1983, $7-28$.

Simon, H.A.: Models of Man - Social and Rational, New York, 1957.

Simon, H.A.: Organizations and Markets, in: Journal of Economic Perspectives, 5, 2, 1991, 25-44.

Snowden, D.: (Senior Consultant on Knowledge Management at IBM) presentation at the EUDOKMA Seminar, Nizza, Dezember 2001.

Soukup, C.: Wissensmanagement. Wissen zwischen Steuerung und Selbstorganisation, Working Paper, Univ. Klagenfurt, 1999.

Spender, J.-C.: Knowing, Managing and Learning. A Dynamic Managerial Epistemology, in: Management Learning, 25/3, 1994, $387-412$. 
Spender, J.-C.: Competitive Advantage from Tacit Knowledge? Unpacking the Concept and its Strategic Implications, in: B. Moingeon and A. Edmondson (Eds.): Organizational Learning and Competitive Advantage, Sage, 1996, 56-73.

Spender, J.-C.: The Dynamics of Individual and Organizational Knowledge, in: C. Eden and J.-C. Spender (Eds.): Managerial and Organizational Cognition. Theory, Methods and Research. Sage: London, 1998, 13 - 39.

Stein, J.: How institutions learn: A socio-cognitive perspective, in: Journal of Economic Issues, 1997, 729-740.

Stewart, T.A.: Intellectual Capital, London, 1997.

Steyer, J.: Die „geführte Unternehmung": Mythos oder Realitat?, in: von Eckardstein, D., H. Kasper und W. Mayrhofer (Eds.): Management. Theorien-Führung-Veränderung, Stuttgart, 1999, 127-159.

Sveiby, K.E.: Wissenskapital - das unentdeckte Vermögen, Landsberg, 1998.

Sydow, J.: Strategische Netzwerke, Wiesbaden, 1992.

Teece, D.J.: The market for know-how and the efficient international transfer of technology, in: The Annals of the Academy of Political and Social Science, November 1981, 8196.

Thiesse, F.: Prozessorientiertes Wissensmanagement, Konzepte, Methode, Fallbeispiele, Dissertationsschrift, Bamberg, 2001.

Tolbert, P.S. and L.G. Zucker: The institutionalization of Institutional Theory, in: S.R. Clegg, C. Hardy and W.R. Nord (Eds.): Handbook of Organization Studies, London, 1996, 175-190.

Tsang, E.W.: Organizational Learning and the Learning Organization: A Dichotomy Between Descriptive and Prescriptive Research, in: Human Relations, 50/1, 1997, 73 - 88.

Tsoukas, H.: What is Organizational Knowledge?, in: Journal of Management Studies, 38:7, November 2001, 973-993.

Tsoukas, H.: Refining Common Sense: Types of Knowledge in Management Studies, in: Journal of Management Studies, 1994, 761-780.

Tsoukas, H.: The firm as a distributed knowledge system: a constructionist approach, in: Strategic Management Journal, 17, (Winter Special Issue) 1996, 11-15.

Türk, K.: Organisation als Institution der kapitalistischen Gesellschaftsformation, in: Ortmann, G.; J. Sydow; K. Türk (Eds.): Theorien der Organisation: Die Rückkehr der Gesellschaft, Opladen, 1997, S. 124-180.

Venters, W.: Literature review for C-Sand: Knowledge Management, Working Paper, 2002. 
Venzin, M., G. von Krogh and L. Roos: Future Research into Knowledge Managment, in: von Krogh, G., Roos, J., Kleine, D. (Eds.): Knowing in Firms: Understanding, Managing and Measuring Knowledge. Gateshead, 1998, 26-66.

Vollmer, H.: Die Institutionalisierung lernender Organisationen. Vom Neoinstitutionalismus zur wissenssoziologischen Aufarbeitung der Organisationsforschung.

Walger, G. und F. Schencking: Wissensmanagement, das Wissen schafft, in: Schreyögg, G. (Hrsg.): Wissen in Unternehmen: Konzepte, Maßnahmen, Methoden, 1. Aufl., Berlin, 2001, 22-39.

Walgenbach, P.: Institutionalistische Ansätze in der Organisationstheorie, in: Kieser, Alfred (Eds.): Organisationstheorien. 3rd edition, Kohlhammer: Stuttgart, 1998, 319-353.

Walgenbach, P.: Neoinstitutionalistische Organisationstheorie, in: Schreyogg, G., P. Conrad und J. Sydow (Eds.): Theorien des Managements. Managementforschung 12, Wiesbaden, 2002, 156-202.

Walsh, J.P. and G.R. Ungeson: Organizational Memory, in: Academic of Management Review, 16, 1991, 57-91.

Weber, M.: Wirtschaft und Gesellschaft, Grundriß der verstehenden Soziologie, 5. rev. Aufl., Tübingen, 1972.

Weick, K.E.: Sensemaking in Organizations, California, 1995.

Weick, K.E. and K. Roberts: Collective Mind in Organizations: Heedful Interrelating on Flight Decks, in Administrative Science Quarterly, 38, 1993, 357-381.

Wenger, E.C.: Communities of Practice - Learning, Meaning, and Identity, Cambridge, 1998.

Wenger, E.C. and W.M. Snyder: Communities of Practice: The organizational frontier, in: Harvard Business Review, January-February, 2000, 139-145.

Wernerfelt, B.: A Resource-based View of the Firm, in: Strategic Management Journal 1984, No.5, 171-180.

Wiegand, M.: Prozesse organisationalen Lernens, Wiesbaden, 1996.

Wieland, J.: Die Neue Organisationsökonomik: Entwicklung und Probleme der Theoriebildung, in: Ortmann, G.; J. Sydow; K. Turk (Eds.): Theorien der Organisation: Die Rückkehr der Gesellschaft, Opladen, 1997, 35-66.

Williamson, O.E.: Markets and Hierarchies: Analysis and Antitrust Implications. A Study in the Economics of Internal Organization, New York, 1975.

Williamson, O.E.: The Economic Institutions of Capitalism. Firms, Markets, Relational Contracting, New York, 1985.

Williamson, O.E.: Transaction Cost Economics, in: Schmalensee, R. und R.D. Willig

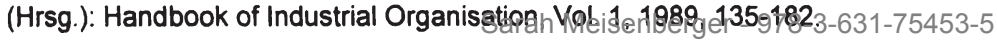


Williamson, O.E. (1990a): A Comparison of Alternative Approaches to Economic Organisation, in: Journal of Institutional and Theoretical Economics, Vol. 146, 1990, 61-71.

Williamson, O.E. (1990b): Die okonomischen Institutionen des Kapitalismus: Unternehmen, Mărkte, Kooperationen, Tübingen, 1990.

Williamson, O.E. (Eds.): Organization Theory: From Chester Barnard to the Present and Beyond, Oxford, 1995.

Willke, H.: Systemisches Wissensmanagement, Stuttgart, 1998.

Willke, H.: Wissensgesellschaft, Vortragsreihe des Instituts für Soziologie, Univ. Wien, 1999.

Willke, H.: Systemisches Wissensmanagement, 2.Aufl., Stuttgart, 2001.

Zand, D.E.: Managing the Knowledge Organization, in: Drucker, P. (Ed.): Preparing Tomorrows Business Leaders Today, Englewood Chliffs, 113-136.

Zander, U. and B. Kogut: Knowledge and the Speed of the Transfer and Imitation of Organizational Capibilities: An empirical test, in: Organization Science, Vol 6, 1995, 76-92.

Zuboff, Sh.: In the Age of the Smart Machine: The Future of Work and Power, 1988.

Zucker, B. und Ch. Schmitz: Wissen gewinnt. Innovative Unternehmensentwicklung durch Wissensmanagement, Düsseldorf/Berlin, 2000.

Zucker, L. G.: Institutional Theories of Organization, in: Annual Review of Sociology, 1987, 443-464. 


\section{Forschungsergebnisse der Wirtschaftsuniversität Wien}

Herausgeber: Wirtschaftsuniversität Wien vertreten durch a.o. Univ. Prof. Dr. Barbara Sporn

Band 1 Stefan Felder: Frequenzallokation in der Telekommunikation. Ökonomische Analyse der Vergabe von Frequenzen unter besonderer Berücksichtigung der UMTS-Auktionen. 2004.

Band 2 Thomas Haller: Marketing im liberalisierten Strommarkt. Kommunikation und Produktplanung im Privatkundenmarkt. 2005.

Band 3 Alexander Stremitzer: Agency Theory: Methodology, Analysis. A Structured Approach to Writing Contracts. 2005.

Band 4 Günther Sedlacek: Analyse der Studiendauer und des Studienabbruch-Risikos. Unter Verwendung der statistischen Methoden der Ereignisanalyse. 2004.

Band 5 Monika Knassmüller: Unternehmensleitbilder im Vergleich. Sinn- und Bedeutungsrahmen deutschsprachiger Unternehmensleitbilder - Versuch einer empirischen (Re-)Konstruktion. 2005.

Band 6 Matthias Fink: Erfolgsfaktor Selbstverpflichtung bei vertrauensbasierten Kooperationen. Mit einem empirischen Befund. 2005.

Band 7 Michael Gerhard Kraft: Ökonomie zwischen Wissenschaft und Ethik. Eine dogmenthistorische Untersuchung von Léon M.E. Walras bis Milton Friedman. 2005.

Band 8 Ingrid Zechmeister: Mental Health Care Financing in the Process of Change. Challenges and Approaches for Austria. 2005.

Band 9 Sarah Meisenberger: Strukturierte Organisationen und Wissen. 2005.

www.peterlang.de 


\section{Theorien des}

\section{Wissensmanagements}

Frankfurt am Main, Berlin, Bern, Bruxelles, New York, Oxford, Wien, 2004. 266 S., zahlr. Abb., Tab. und Graf.

ISBN 3-631-51999-0 - br. € 45.-*

Der Band hat die Nutzbarmachung von Wissensmanagement zur

Optimierung und Beschleunigung von betrieblichen Innovationsprozessen zum Gegenstand. Ist die gezielte Nutzung von Wissen Voraussetzung für Innovation und Entwicklung? Welche theoretischen Ausgangspunkte müssen dabei berücksichtigt werden? Es werden neue interdisziplinäre Perspektiven des Wissensmanagements eröffnet. Dabei werden nicht nur unterschiedliche Zugänge und Konzepte vorgestellt, sondern auch

(1) Zusammenhänge und Interdependenzen untersucht. Die Leserin und der Leser erhalten in dem Band einen Einblick vor und hinter die Kulissen der aktuellen theoretischen Diskussion zum Wissensmanagement. Sie finden hier Anregungen für die Übertragung auf Ihre Praxis.

Aus dem Inhalt: Zugänge zum Wissensmanagement · Soziologie · Wettbewerb - Marketing · Human Resource - Kreativität - Innovation . System $\cdot$ Interdependenzen

Frankfurt am Main - Berlin - Bern - Bruxelles - New York - Oxford - Wien Auslieferung: Verlag Peter Lang AG

Moosstr. 1, $\mathrm{CH}-2542$ Pieterlen

Telefax 0041 (0) $32 / 3761727$

*inklusive der in Deutschland gültigen Mehrwertsteuer Preisänderungen vorbehalten

Homepage http://unw.peterlang.de 